\title{
Activation of the influenza virus hemagglutinin by type II transmembrane serine proteases
}

\author{
Dissertation \\ for the award of the degree \\ "Doctor of Philosophy" \\ Division of Mathematics and Natural Sciences \\ of the Georg-August-Universität Göttingen
}

within the doctoral program Emerging Infectious Diseases

of the Georg-August University School of Science (GAUSS)

submitted by

Pawel Zmora

from Szprotawa, Poland

Göttingen, 2015 


\section{Thesis Committee}

Prof. Dr. Stefan Pöhlmann, Infection Biology Unit, German Primate Center

Prof. Dr. Dr. Claus-Peter Czerny, Division of Microbiology and Animal Hygiene,

Department of Animal Sciences, Institute of Veterinary Medicine, Georg-August University

Göttingen

Dr. Mikhail Matrosovich, Institute of Virology, Philipps University, Marburg

\section{Members of the Examination Board}

Referee: Prof. Dr. Stefan Pöhlmann, Infection Biology Unit, German Primate Center

$2^{\text {nd }}$ Referee: Prof. Dr. Dr. Claus-Peter Czerny, Division of Microbiology and Animal Hygiene, Department of Animal Sciences, Institute of Veterinary Medicine, Georg-August University Göttingen

\section{Further members of the Examination Board}

Dr. Mikhail Matrosovich, Institute of Virology, Philipps University, Marburg

Prof. Dr. Carsten Lüder, Institute for Medical Microbiology, University Medical Center, Georg-August-University Göttingen

Prof. Dr. Lutz Walter, Primate Genetics Laboratory, German Primate Center, Göttingen Prof. Dr. Martin Oppermann, Institute for Cellular and Molecular Immunology, University Medical Center, Georg-August-University Göttingen

Date of oral examination: 26.11.2015 


\section{Declaration}

I hereby declare that this submission is my own work and that, to the best of my knowledge and belief, it contains no materials previously published or written by another person nor material which to a substantial extent has been accepted for the award of any other degree of the university or other institute of higher education, except where due acknowledgment has been made in the text.

Göttingen, 13.10.2015

Pawel Zmora 


\section{Contents}

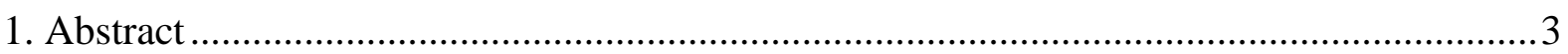

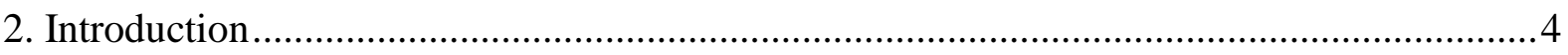

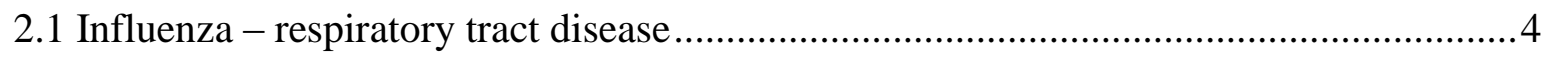

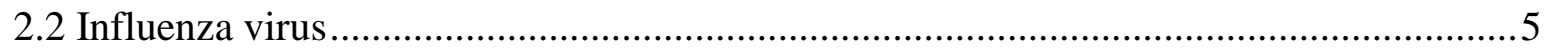

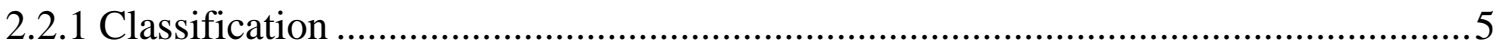

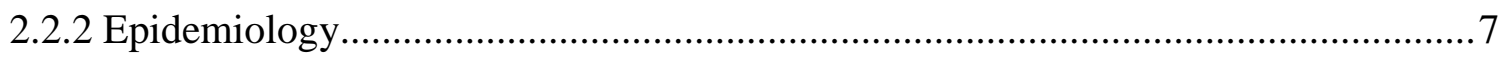

2.2.3 Current approaches to prevent and treat influenza …………………………….... 10

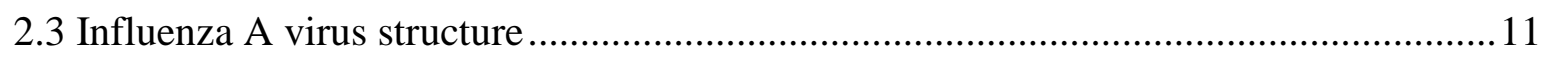

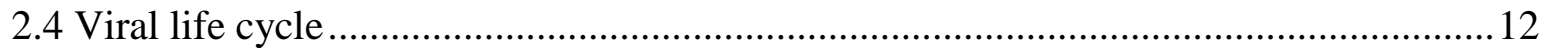

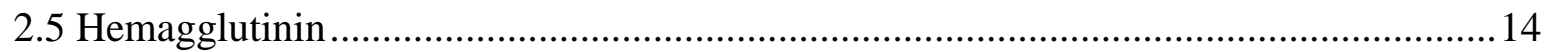

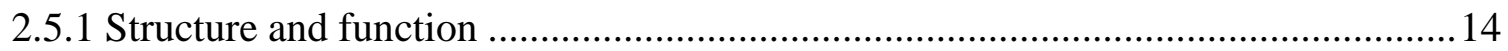

2.5.2 Class I fusion proteins and membrane fusion mechanism ..................................... 16

2.5.3 Proteolytic cleavage as a requirement of membrane fusion .................................... 18

2.6 Type II transmembrane serine proteases .............................................................2

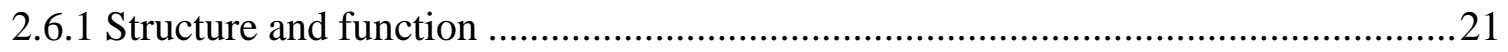

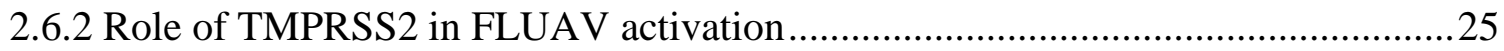

2.6.3 Role of type II transmembrane serine proteases in influenza virus replication:

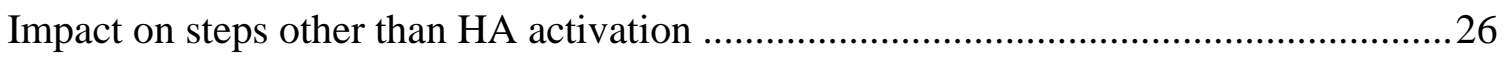

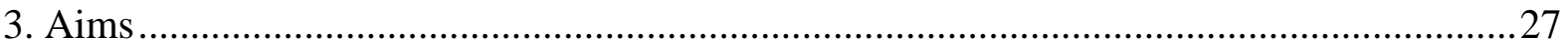

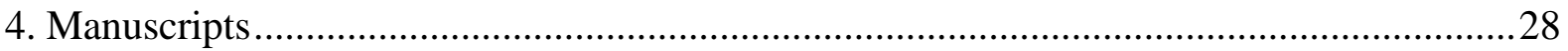

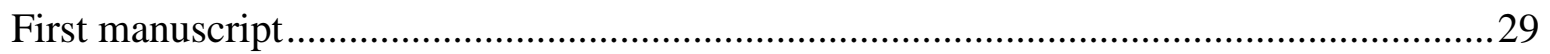

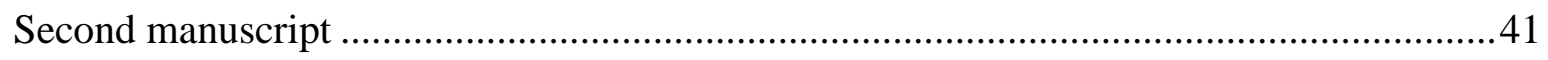

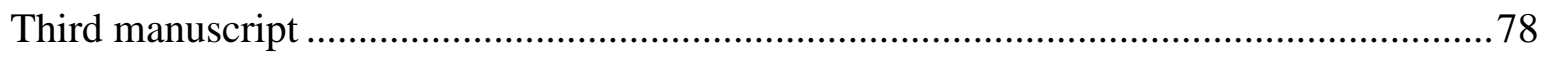

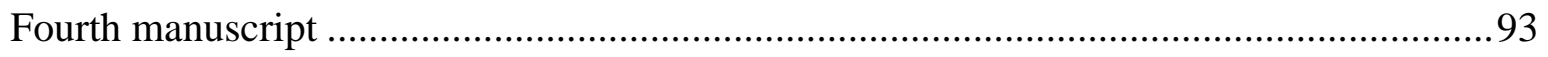

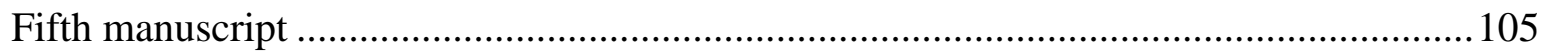

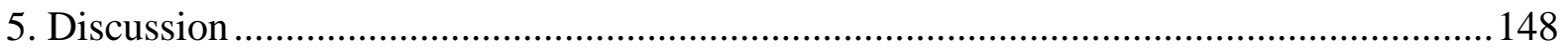

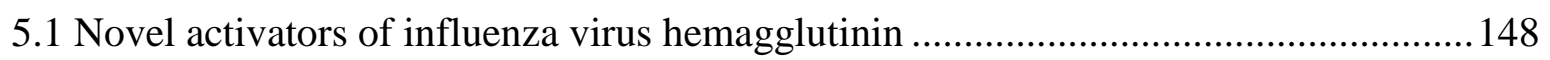

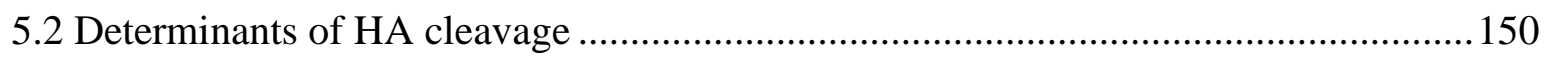

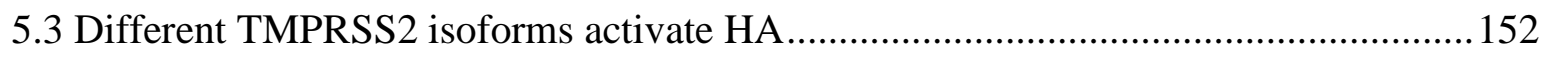

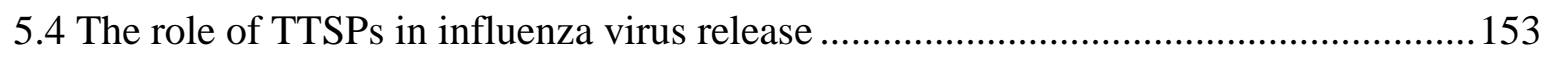




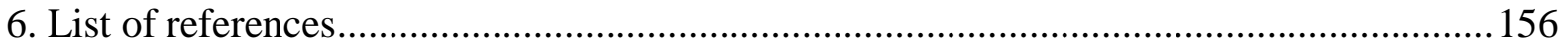

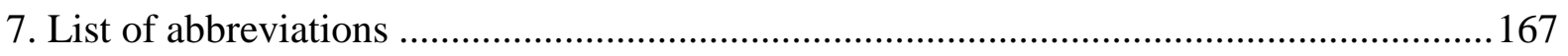

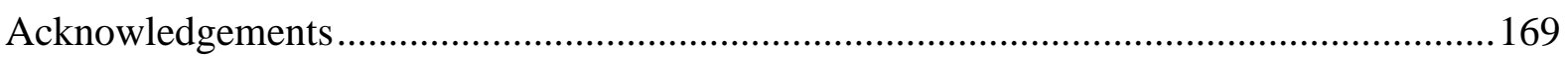

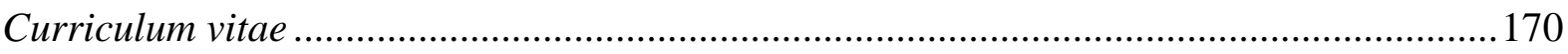




\section{Abstract}

The influenza virus hemagglutinin (HA) mediates viral entry into target cells. HA is synthesized as an inactive precursor and depends on proteolytic processing by host cell proteases to become active. TMPRSS2, a member of the type II transmembrane serine protease (TTSP) family, can cleave and activate HA in cell culture and was recently shown to be essential for spread and pathogenesis of influenza viruses of the H1N1 subtype in mice. In contrast, an $\mathrm{H} 3 \mathrm{~N} 2$ influenza virus was less dependent on TMPRSS2, implying that this virus may hijack other TTSPs for its activation. Moreover, studies with an animal coronavirus suggested that TMPRSS2 could impact virus infection at stages other than viral entry into cells. Therefore, the central goals of this thesis were: i) to identify TTSPs which activate HA; ii) to identify molecular determinants which control the ability of TTSPs to cleave and activate HA; iii) to determine whether TMPRSS2 promotes influenza virus infection by processes other than HA activation.

The results of the present thesis show that DESC1 and MSPL, members of the TTSP family, activate diverse influenza viruses, while the related proteases TMPRSS11F, prostasin, TMPRSS11B, TMPRSS9 and TMPRSS10 fail to do so. Additionally, it was demonstrated that TTSPs which activate HA also colocalize with HA at or close to the cell membrane while this phenotype was not observed with enzymes unable to activate HA. Mutagenic analysis revealed that the stem region of TTSPs controlled their ability to colocalize and to activate HA. Moreover, evidence was obtained that targeting the scavenger receptor A domain present in the stem region of certain HA-activating TTSPs may block HA processing. Finally, evidence was obtained that TMPRSS2 and other HA-activating TTSPs can antagonize the antiviral host cell factor tetherin and might thereby promote influenza virus release form tetherin expressing cells. In sum, the present study identified two TTSPs as HA activators, and defined the stem region of TTSPs as a potential target for antiviral strategies. Finally, it was shown that TTSPs may promote influenza virus spread via two mechanisms, HA activation and tetherin inactivation.

Keywords: influenza virus, hemagglutinin, type II transmembrane serine proteases, proteolytic cleavage, TMPRSS2, DESC1, MSPL, tetherin 


\section{Introduction}

Data from the World Health Organization (WHO) show that the main causes of global mortality are: i) ischemic heart disease, ii) stroke, iii) chronic obstructive pulmonary diseases, iv) respiratory tract infections, v) trachea, bronchus and lung cancer, vi) HIV/AIDS, vii) diarrheal diseases, viii) diabetes mellitus, ix) road injuries and $\mathrm{x}$ ) hypertensive heart diseases (www.who.int, 2015). Respiratory tract infections were responsible for 3.1 million deaths (5\% of total deaths) worldwide in 2012, with influenza being responsible for approximately $20 \%$ of these death (www.who.int, 2015). However, it should be underlined that the numbers differ between low- and high-income countries. In low-income countries, for instance Afghanistan and Kenya, lower respiratory tract infections were the main cause of death, killing 91 persons per 100,000 population. In contrast, in high income countries like Germany or the United States of America (USA), the main cause of death was ischemic heart disease and lower respiratory infections were responsible for the death of less than 31 persons per 100,000 population (www.who.int, 2015). Additionally, it should be underlined that influenza has a significant socio-economic impact, and causes high financial loss.

\subsection{Influenza - respiratory tract disease}

The term "respiratory tract infection" refers to infections of the upper or lower respiratory tract by bacteria, fungi or viruses. Symptoms associated with upper respiratory infection include the common cold, sore throat and tonsillitis while lower respiratory infections mainly induce bronchitis and pneumonia. Influenza, or shortly 'flu', can affect both parts of respiratory tract (Clark and Lynch, 2011; Govaert et al., 1998). According to the US Centers for Disease Control and Prevention (www.cdc.gov, 2015), influenza or influenzalike illness is defined by a temperature higher than $37.8^{\circ} \mathrm{C}$ and either cough or sore throat in the absence of a known cause other than influenza virus (Harper et al., 2004). The WHO states that influenza is characterized by a sudden onset of high fever together with other symptoms, which differ between adults and children (www.who.int, 2015). High temperature, dry cough, sore throat and nasal congestion or runny nose, are common in both adults and children suffering from influenza. In contrast, headache and myalgia are more common in adults while vomiting and diarrhea are mainly observed in children (Clark and Lynch, 2011; Khandaker et al., 2011; Punpanich and Chotpitayasunondh, 2012; Tregoning and Schwarze, 2010). Most patients recover from influenza symptoms within a week, but influenza can be associated with severe respiratory, cardiovascular and others complications, like acute 
bronchitis, secondary bacterial pneumonia and otitis media (i.e. inflammatory diseases of middle ear) (Clark and Lynch, 2011; Harper et al., 2004; Punpanich and Chotpitayasunondh, 2012). The following groups are at high risk for complications: adults aged 65 years or older, children younger than 2 years, pregnant women, individuals with chronic pulmonary or cardiovascular or hematologic or metabolic disorders, and persons with immunosuppression caused by either diseases, for instance HIV/AIDS, or medication (Clark and Lynch, 2011; Harper et al., 2004).

\subsection{Influenza virus}

Influenza virus, the causative agent of influenza was first described in 1933 by Smith and colleagues. They showed that a filterable agent isolated from an individual with influenza symptoms caused an influenza-like illness in experimentally inoculated ferrets (Smith et al., 1933). Subsequently, it was shown that influenza viruses can be propagated in embryonated chicken eggs, contain enzymes, i.e. neuraminidase, and recombine their genomes at high frequency.

\subsubsection{Classification}

In a phylogenetic-based taxonomy, influenza viruses belong to the category 'RNA viruses', subcategory 'negative-sense single-stranded RNA viruses', order 'Mononegavirales', family 'Orthomyxoviridae'. There are three genera of influenza viruses: influenza A virus (FLUAV), influenza B virus (FLUBV) and influenza C virus (FLUCV). The FLUAV can be additionally divided into subtypes on the basis of antigenic properties of two viral surface glycoproteins: hemagglutinin (HA, subtypes H1-H18) and neuraminidase (NA, subtypes N1-N11). Examples for influenza virus subtypes are FLUAV H1N1 (for instance the virus responsible for the 'swine flu' pandemic in 2009), FLUAV H3N2, FLUAV H5N1 (the viruses responsible for 'avian flu'), FLUAV H17N10 and H18N11 (subtypes recently identified in bats). Further characterization of influenza virus strains includes information on the geographic origin, strain number, year of isolation and virus subtype, i.e. influenza virus A/Puerto Rico/8/34 (H1N1) is the $8^{\text {th }}$ strain of H1N1 subtype, isolated in Puerto Rico in 1934.

\section{Influenza A virus}

FLUAV can infect people, birds, pigs, horses, whales, seals and other mammals. It is believed that water fowl is the natural reservoir of FLUAV (Horimoto and Kawaoka, 2005; 
Palese, 2004; Schrauwen et al., 2014; Webster et al., 1995). However, FLUAV were recently detected in Guatemala yellow-shouldered bats and the role of bats in the influenza zoonosis is currently under intensive investigation (Tong et al., 2012; Tong et al., 2013). On the basis of molecular genetics and pathogenesis criteria, low pathogenic avian influenza viruses (LPAIV) and highly pathogenic avian influenza viruses (HPAIV) can be discriminated. Most of the known avian influenza viruses are LPAIV and induce no symptoms or mild disease in birds. In contrast, HPAIV cause severe disease with high mortality in poultry (Kuiken, 2013; Sonnberg et al., 2013; Spackman, 2014). LPAI viruses may evolve into HPAIV and cause disease outbreaks in poultry (Sonnberg et al., 2013). Humans can be infected with both with LPAIV and HPAIV. However, infection requires direct contact with infected sick or dead birds. Avian FLUAV of the following HA subtypes are known to infect both birds and people: H5, H7 and H9 (El Zowalaty et al., 2013; Herfst et al., 2014). Infection with HPAIV of the H5N1 subtype causes severe and frequently fatal disease and H5N1 viruses are currently circulating in Asia and Europe (El Zowalaty et al., 2013). Mild to severe, as well as fatal illness in humans have been observed upon infection with H7N7 (HPAIV) or H7N9 (LPAIV) viruses (Puzelli et al., 2014; Shi et al., 2013). On the other hand, only 13 cases of infection with H9N7 avian FLUAV were reported, mainly recorded in Southern China and Hong Kong, and all patients fully recovered (Huang et al., 2015).

\section{Influenza B virus}

FLUBV is known to infect humans and seals. Due to this limited host range, it is believed that FLUBV can cause epidemics but not pandemics. Moreover, influenza caused by FLUBV is frequently less severe in comparison to influenza upon FLUAV infection (Clark and Lynch, 2011; Paul Glezen et al., 2013). The virion structure and replication cycle of FLUBV is similar to that of FLUAV. In contrast to FLUAV, FLUB viruses are a mainly homogenous group, which started to diverge into two antigenically distinct lineages (Victoria and Yamagata lineages) in the 1970s (Biere et al., 2010). The reduced diversity of FLUBV compared to FLUAV reflects slower evolution (Nobusawa and Sato, 2006).

\section{Influenza C virus}

Infections with FLUCV are much less frequent compared to FLUAV and FLUCV. They only cause mild illness, mainly in children (Clark and Lynch, 2011), and the viruses do not have pandemic potential. In contrast to FLUAV (genome and replication strategy are described in detail below) and FLUBV, FLUCV contain only 7 RNA segments, which encode 9 proteins 
(Muraki and Hongo, 2010). Moreover, instead of HA and NA present in FLUAV and FLUBV (which are described in the chapter 3.3 and 3.5), FLUCV harbor a single surface glycoprotein, hemagglutinin-esterase, which mediates virus-cell fusion and plays a role in virus release (Herrler and Klenk, 1991).

\subsubsection{Epidemiology}

New epidemic FLUAV strains can emerge every 1 to 2 years and infection with these viruses is believed to be responsible for 3-5 million cases of severe illness every year (Palese, 2004). The reason for the constant emergence of new FLUAV is the continuous acquisition of point mutations in NA and particularly HA (the function of these proteins is described in detail in the next paragraphs) (Horimoto and Kawaoka, 2005), a process termed antigenic drift. These mutations allow FLUAV to evade the humoral immune response and to constantly circulate in population with preexisting immunity (Medina and García-Sastre, 2011). A hallmark of influenza viruses is their segmented RNA genome, as described below. The exchange of entire genomic segments (reassortment) between different FLUAV can allow the emergence of antigenically novel viruses, termed antigenic shift, against which the population is immunologically naïve (Horimoto and Kawaoka, 2005). A new influenza pandemic might be the consequence. In contrast to the annual epidemics, pandemics occur every 10 to 50 years (Potter, 2001). However, their consequences might be much more severe than influenza epidemics, as discussed below.

\section{Influenza epidemics (Seasonal flu)}

Each year, approximately $10 \%$ of the world's population is infected with influenza viruses, and influenza-related complications cause 250.000-500.000 deaths. Moreover, influenza cases are associated with 87.1 billion USD of direct costs in the USA alone (Molinari et al., 2007). In the northern hemisphere, influenza waves are observed in fall and winter and are caused by the currently circulating FLUAV and FLUBV. In 2014-2015, in the USA the influenza season started at the $44^{\text {th }}$ week of 2014 and lasted until the $15^{\text {th }}$ week of 2015 (Figure 1). The majority of cases were due to infection with FLUAV of the H1N1 or H3N2 subtype, and towards the end of the season the number of patients with FLUBV infection increased (Figure 1). Collectively, seasonal flu follows predictable seasonal patterns, occurs annually and usually causes big impact on society due to the pre-existing immunity, built up from previous exposure with similar strains of flu. 
Influenza Positive Tests Reported to CDC by U.S. WHO/NREVSS Collaborating Laboratories, National Summary, 2014-15

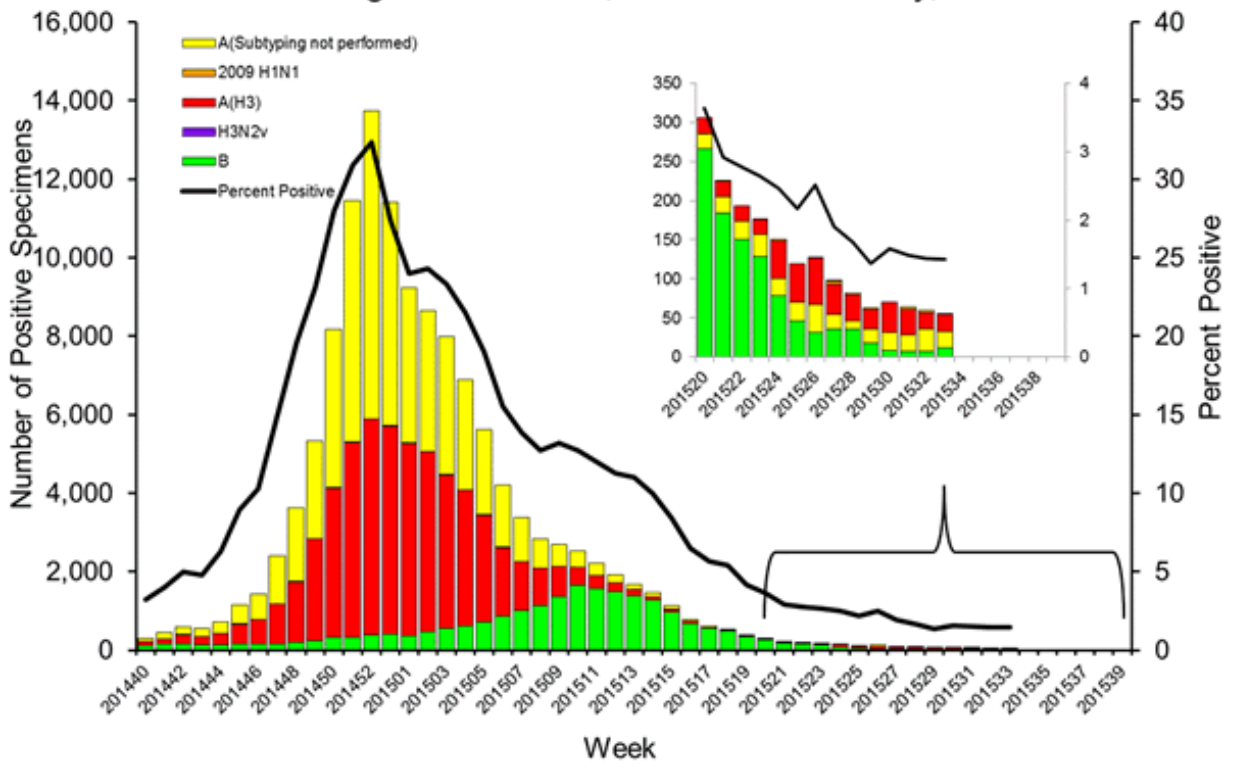

Figure 1. Influenza surveillance. Depicted are numbers of positive specimens tested for the indicated influenza type (e.g. influenza virus B) and subtype (e.g. influenza virus A H3) as well as the percentage of positives among specimens analyzed during the influenza season 2014-2015 in the USA (FluView. A weekly Influenza Surveillance Report, www.cdc.gov).

\section{Influenza pandemics}

\section{Spanish flu}

The Spanish influenza occurred in 1918-1919 and is described as one of three most destructive human pandemics, jointly with the plague of Justinian and the Black Death, during which deaths in hospitals may have exceeded 25\% per night (Potter, 2001). The 1918 influenza pandemic was caused by an H1N1 virus of unknown origin, although, some data suggest that the virus evolved from an avian virus with or without adaptation to an intermediate host (Schrauwen et al., 2014). In less than one year, the virus infected $50 \%$ of the world's population and killed 40-50 million people worldwide (Palese, 2004). Unlike any other recorded influenza pandemic, the Spanish influenza mainly affected patients aged 2040 , and the reasons for this are still unclear. One explanation might be lack of previous exposure to H1N1 viruses, since a H3 virus caused a pandemic in 1898-1900 as well as subsequent epidemics (Schrauwen et al., 2014). In contrast, older people might have previously been exposed to a H1 virus which caused a pandemic in 1830-1833 and subsequently circulated as a seasonal strain (Potter, 2001). As a consequence, the death rate decreased in people older than 40, only to rise again in the elderly, as is typical for influenza. 


\section{Asian flu}

The 1957 the 'Asian flu' was caused by an H2N2 virus, which contained HA, NA and polymerase basic 1 (PB1) genomic segments from an avian $\mathrm{H} 2 \mathrm{~N} 2$ virus and the remaining segments from the H1N1 virus circulating at this time (a descendant of the $1918 \mathrm{H} 1 \mathrm{~N} 1$ virus). The pandemic claimed 1-2 million lives, and a fatal outcome was predominantly observed in children and the elderly, much in contrast to the 1918 pandemic (Potter, 2001). Most deaths were due to the secondary bacterial pneumonia, as commonly observed for influenza.

\section{Hong Kong flu}

In 1968, a reassortant H3N2 virus emerged with the HA and PB1 genomic segments from an avian influenza virus and the remaining segments from the previously circulating $\mathrm{H} 2 \mathrm{~N} 2$ virus. Due to the prior immunity in the human population against N2 neuraminidase, the pandemic was characterized by lower morbidity and mortality as compared to the 1957 pandemic (Potter, 2001), although it was estimated that almost 1 million people died because of influenza and secondary bacterial pneumonia.

\section{Russian flu}

The fourth influenza pandemic of the 20st century unfolded in 1977 and was again caused by an H1N1 influenza virus. The virus caused disease mostly in younger people, born after 1950, due to pre-existing immunity in the older population, resulting from previous exposure to H1N1 FLUAV. The Russian H1N1 FLUAV strain was closely related to viruses circulating before 1950 (Potter, 2001), and its origins are unclear. The virus might have been accidentally released from a laboratory freezer or might have been introduced into the population in the course of vaccine trials with military recruits conducted in East Russia (Palese, 2004; Potter, 2001).

\section{Swine flu}

The first influenza pandemic of the 21st century started in spring 2009 in Mexico and within a few months the new virus, again of the H1N1 subtype, spread globally, resulting in 100.000 to 300.000 deaths (York and Donis, 2013). The virus caused disease in a disproportionately high frequency among young adults, while people over 65 years old might have been partially protected, since the virus was relatively close to the H1N1 virus circulating before 1957 (Schultz-Cherry et al., 2013). In opposite to the 1918 FLUAV 
responsible for the Spanish flu, the 2009 H1N1 FLUAV was a reassortant with NA and M genes from the 'Eurasian' swine influenza virus lineage while the rest of the genes originated from a triple reassortant virus, which contained genes of human, avian and swine origin (Schultz-Cherry et al., 2013; York and Donis, 2013).

\subsubsection{Current approaches to prevent and treat influenza}

Influenza is frequently treated with supportive therapy, which includes bed rest, adequate hydration and administration of anti-fever drugs and painkillers, i.e. acetylsalicylic acid or ibuprofen. Additionally, nasal sprays, water vaporization and cough suppressants can be included. Antibiotic treatment should be applied only when secondary bacterial pneumonia is diagnosed (Harper et al., 2004). Specific anti-influenza therapy is available. However, it should be reserved for high risk patients or individuals with more severe disease course (Harper et al., 2004). Currently, there are two groups of antiviral drugs against flu: the M2 ion channel blockers (amantadine and rimantadine) and NA inhibitors (oseltamivir and zanamivir).

Amantadine as well as rimantadine (an amine derivate of adamantine) block the viral M2 ion channel protein and thereby prevent from release of viral genetic material to the cytoplasm and thus suppress the viral replication (the viral structure and replication are described in the next paragraphs) (Fleming, 2001; Gu et al., 2013; Krol et al., 2014; Saladino et al., 2010). However, viruses rapidly acquires resistance mutations and up to $92 \%$ of the currently circulating H3N2 strains are amantadine/rimantadine resistant (Zhang et al., 2013), and the pandemic 2009 swine flu strain was found to be completely resistant to adamantine derivatives (Baranovich et al., 2015). Therefore, the WHO does not recommend the use of M2 ion channel blockers as anti-influenza drugs.

The second class of anti-influenza drugs are NA inhibitors (oseltamivir and zanamivir), which inhibit release of progeny virions from infected cells. These compounds block the catalytic center of NA and thereby prevent FLUAV transmission from infected cells to neighboring cells (Krol et al., 2014; Shtyrya et al., 2009). The administration of these drugs during the first two days of influenza onset can significantly reduce illness duration and severity (Kidd, 2014; Saladino et al., 2010). The catalytic center of NA is highly conserved between different influenza strains and one would expect mutations in this domain to alter viral fitness (Shtyrya et al., 2009). Nevertheless, FLUAV strains resistant to oseltamivir emerged (for instance the 2009 pandemic FLUAV strain was resistant to oseltamivir) and 
were efficiently transmitted, due to the presence of compensatory mutations, which allowed robust viral spread despite oseltamivir resistance (Baek et al., 2015; Baranovich et al., 2015; Duan et al., 2014; Kiso et al., 2010). In contrast, zanamivir resistance has been rarely reported to date (Thorlund et al., 2011).

The most efficient strategy to fight infectious diseases is vaccination. Vaccination can prevent or at least ameliorate morbidity and mortality of diseases like chicken pox, polio, measles and tetanus. In the case of small pox, vaccination even allowed the eradication of the causative agent. Vaccination against influenza is available since the 1930s (Shope, 1936). However, due to constant antigenic drift of circulating viruses vaccination needs to be repeated annually. At present, two types of influenza vaccines are available: trivalent and quadrivalent vaccines, which can contain either inactivated (whole inactivated viruses, split viruses, viral subunits) or live attenuated (less virulent) viruses (Soema et al., 2015). The trivalent influenza vaccines consist of two FLUAV strains and one FLUBV strain, while the quadrivalent vaccines contain combinations of two FLUAV and two FLUBV strains (Soema et al., 2015). In both cases, the vaccines are designed to stimulate the immune system to develop an adaptive response against viruses predicated to be circulating during the next influenza season.

\subsection{Influenza A virus structure}

Influenza virus particles are enveloped and of spherical or filamentous form. The envelope contains that surface proteins HA and NA, which are detectable as glycoprotein 'spikes' upon electron-microscopic analysis, and the ion channel M2 (Fig. 2A) (Das et al., 2010). The envelope encloses the virion interior which contains eight segments (seven in the case of FLUCV) of the negative-sense single-stranded RNA genome. The genomic segments encode three subunits of the viral RNA-dependent RNA polymerase (polymerase acidic protein, PA; polymerase basic protein 1, PB1 and polymerase basic protein 2, PB2), the viral surface glycoproteins HA and NA, the ion channel M2, the nucleoprotein (NP), matrix proteins (M1 and M2 ion channel) and two non-structural proteins (NS1 and NS2). The second FLUAV RNA genomic segment, except of PB1, encodes two additional protein: PB1F2 and $\mathrm{N} 40$, which play role in promoting virus replication and increase pathogenesis (Chakrabarti and Pasricha, 2013). The viral RNA is associated with the viral RNA polymerase (PB1, PB2 and PA) and is coated with multiple nucleoproteins (NPs) to form the so called viral ribonucleoprotein (vRNP) complex. The vRNPs are surrounded by a layer of the matrix 
protein, M1, which is located underneath the viral envelope. The lipid layer originated from the host cell during and was acquired during budding of progeny particles from infected cells (Fig. 2B).
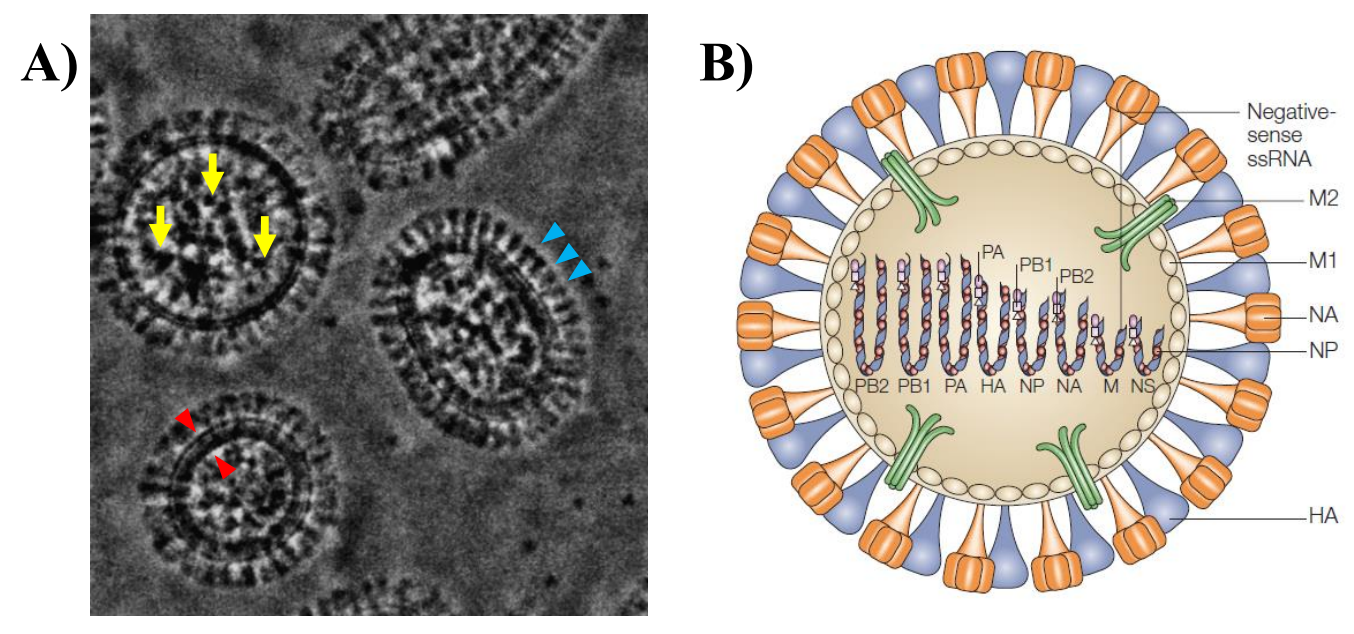

Figure 2. Influenza A virus particle. A) A Zernike phase contrast electron micrograph of iceembedded FLUAV. Spherical and elongated virions in which the envelope (between red arrowheads), ribonucleoproteins complexes (yellow arrows) and surface glycoproteins (blue arrowheads) are indicated (Figure was modified from Yamaguchi et al., 2008). B) Scheme of FLUAV virions. The virus particle contain eight ribonucleoprotein complexes (vRNPs), which harbor negative sense single stranded RNA segments, associated with the nucleoprotein (NP) and three polymerase proteins (PA, $\mathrm{PB} 1, \mathrm{~PB} 2)$. The vRNPs are connected via the matrix protein (M1) with the viral envelope, which is spiked with surface glycoproteins: hemagglutinin (HA), neuraminidase (NA). In addition, the M2-ion channel protein is inserted into the envelope (Figure was taken from Harimoto and Kawaoka, 2005).

\subsection{Viral life cycle}

The viral life cycle commences with attachment of virions to target cells (Luo, 2012). This process is mediated by the HA protein, which binds to $\alpha-2,3$ - (avian viruses) or $\alpha-2,6$ linked (human viruses) sialic acids on cellular glycoproteins or glycolipids (Step 1, Figure 1) (Matrosovich et al., 2009). Subsequently, virus is transported to the cell interior by endocytosis (Step 2, Figure 3) (Luo, 2012). Next, the acidification of endosomes triggers conformational changes in HA, which bring about fusion of the viral and the endosomal membrane (Step 3, Figure 3) (Sriwilaijaroen and Suzuki, 2012). Moreover, proton transport into the virion interior via the ion channel M2 abrogates the interaction between vRNPs and the M1 matrix protein and the release if vRNPs into the host cell cytoplasm (Step 4, Figure 3) (Sun, 2013). After transport to the nucleus, synthesis of viral mRNAs starts (Step 5, Figure 3) 
followed by protein expression (Step 6, Figure 3) (Das et al., 2010). The nascent polypeptides of the viral surface proteins HA and NA are imported and N-glycosylated in the endoplasmic reticulum. The $\mathrm{N}$-glycans are trimmed in the Golgi apparatus and the mature proteins are transported to the cell surface, where the HA of human influenza viruses can be proteolytically processed by type II transmembrane serine proteases (Step 7, Figure 3) (Das et al., 2010). In the nucleus, newly formed viral RNAs associate with NP and viral polymerase subunits to form vRNPs, which can be exported from the nucleus as a function of nuclear export protein (NEP, also referred to as non-structural protein 2, or NS2) (Step 8, Figure 3) (Das et al., 2010). At the cellular surface, the vRNPS are incorporated into budding viruses via interactions between M1 and the cytoplasmic tails of the viral surface proteins (Step 9, Figure 3) (Ali et al., 2000). Finally, progeny particles are released from the cells and this process is promoted by NA-mediated cleavage of sialic acid residues on cellular and viral factors present at the cell surface (Step 10, Figure 3) (Rossman and Lamb, 2011).

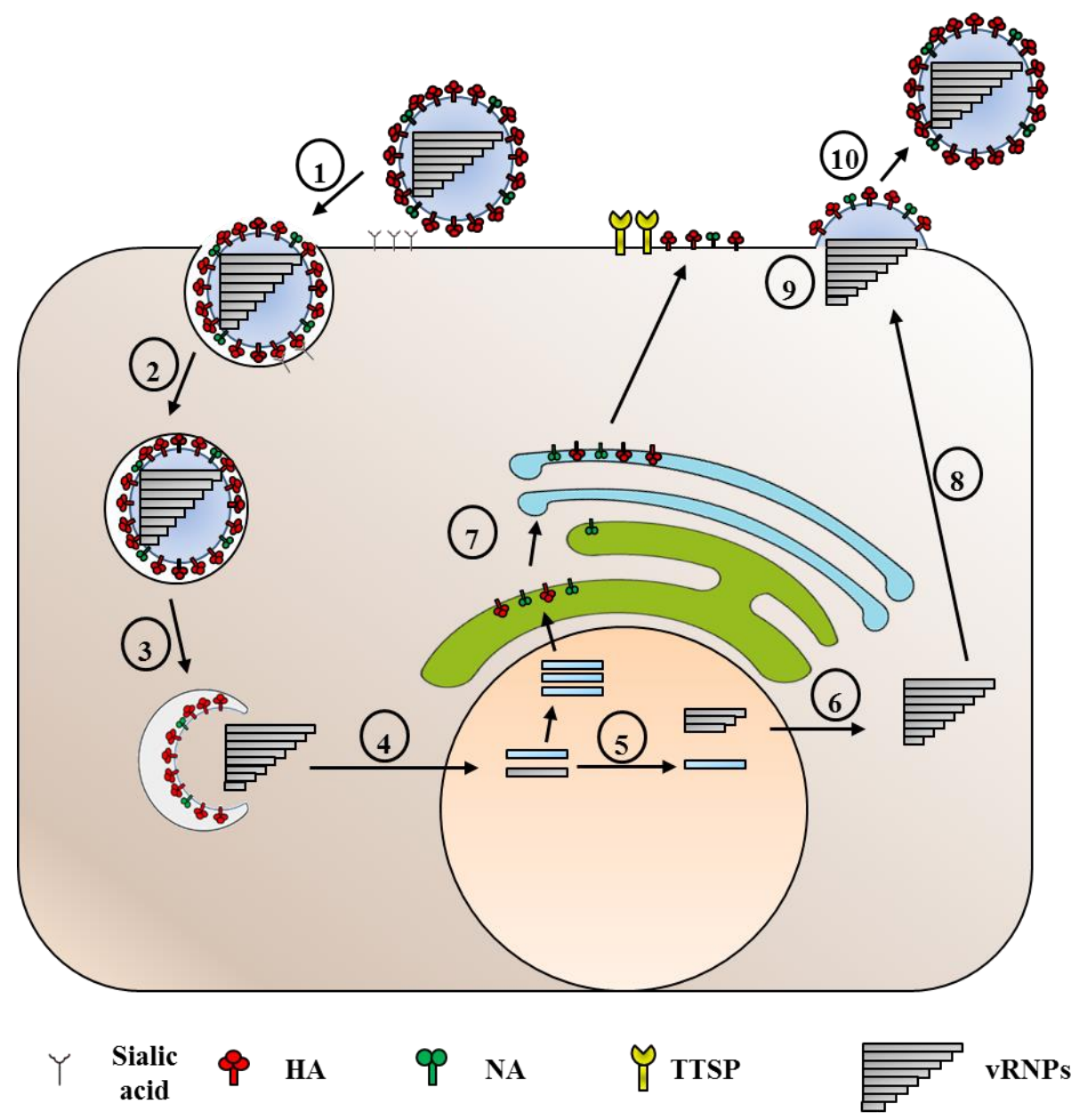

Figure 3. Influenza virus life cycle. (Figure was taken from Zmora and Pöhlmann, 2015) 


\subsection{Hemagglutinin}

\subsubsection{Structure and function}

HA is inserted in the viral envelope, coded by the $4^{\text {th }}$ RNA segment and comprises approximately 560 amino acid residues with a molecular weight of approximately $60 \mathrm{kDa}$ (for unglycosylated HA) (Das et al., 2010; Schrauwen et al., 2014; Sriwilaijaroen and Suzuki, 2012). The molecular weight increases upon $\mathrm{N}$-glycosylation and the increase depends on the number and complexity of N-glycans attached to HA (Sriwilaijaroen and Suzuki, 2012). An N-terminal signal sequence mediates import of the nascent $\mathrm{HA}_{0}$ polypeptide chain into the ER, where the signal sequence is removed, the protein is folded and modified by highmannose N-glycans (Sriwilaijaroen and Suzuki, 2012). In addition, HA homotrimers are formed in the ER. In the Golgi apparatus, the N-glycans are trimmed and HA of HPAIV is proteolytically cleaved into the surface unit, $\mathrm{HA}_{1}$, and the transmembrane unit, $\mathrm{HA}_{2}$ (Bertram et al., 2010b; Bottcher-Friebertshauser et al., 2013). In contrast, cleavage of HA of LPAIV and human influenza viruses is believed to occur during or after transport of the protein to the cell surface (Bertram et al., 2010b), as discussed below. HA is the major target for the neutralizing antibody response and the decoration of HA with $\mathrm{N}$-glycans is believed to mask underlying epitopes from recognition by antibodies (Das et al., 2010). The number of signals for N-glycosylation varies between FLUAV isolates: The HA of the pandemic 1918 FLUAV contains only one $\mathrm{N}$-glycosylation site in the globular head domain at N95, while the seasonal H1N1 FLUAV A/Puerto Rico/8/34 has four N-glycosylation sites: at position N95, N129 or N131, N158 and/or N163 or N165 in the same region (Igarashi et al., 2010). A different seasonal FLUAV strain, A/Texas/36/91, contains five glycans on the HA head: at positions N71, N104, N142, N177 and N286, while the more recent strain A/Solomon Island/2006 possesses four glycans at positions N71, N104, N142 and N177 and shows the evolutionary trend to lose the N-glycosylation site at position N286 (Sun et al., 2013).

The HA is a type I transmembrane protein, i.e. the N-terminal part is located outside the cell while a C-terminal hydrophobic transmembrane domain anchors $\mathrm{HA}$ in the plasma membrane and the viral envelope, respectively (Harrison, 2015; Sriwilaijaroen and Suzuki, 2012; White et al., 2008). The HA cytoplasmic tail, which consists of 10-11 amino acids, is not required for intracellular transport to the plasma membrane (Jin et al., 1994), but it was shown that the lack of the cytoplasmic tail resulted in defective virion morphogenesis and improper genome packaging (Zhang et al., 2000). Additionally, it was also demonstrated that 
palmitoylation of the HA cytoplasmic tail is dispensable for HA-mediated membrane fusion in the case of $\mathrm{H} 3$ subtype HA (Chen et al., 2005). HA has a cylindrical shape (135 A long and 35-70 A wide) and forms 'spikes' on the virion surface, which can be readily visualized via electron microscopy (Fig. 2B) (Yamaguchi et al., 2008). Two major domains have been identified in HA: The globular head domain, which is mainly build up from the HA $\mathrm{H}_{1}$ subunit, and a stalk-like domain, called stem, which contains parts of $\mathrm{HA}_{1}$ and the entire extracellular part of $\mathrm{HA}_{2}$ (Figure 4) (Chen et al., 1998; White et al., 2008). The globular head contains $\mathrm{HA}_{1}$ residues 116-261, which are folded into a jelly-roll motif of eight stranded antiparallel $\beta$ sheets, and harbor sialic acid binding sites at the distal tip, as well as major antigenic regions (Figure 4) (Chen et al., 1998). In contrast, the HA stem contains an elongated central coiledcoil, formed by the non-covalent association of three helices of each monomer (Chen et al., 1998). The surface loop structure at the HA stem domain contains a cleavage site (Figure 4) for host cell proteases and cleavage is required for HA-driven fusion of viral and cellular membranes and thus for viral infectivity (Chen et al., 1998). The proteolytic cleavage induces the repositioning of the highly conserved $\mathrm{N}$-terminus of the $\mathrm{HA}_{2}$ subunit, which encodes the fusion peptide, to the interior of the HA trimer (Chen et al., 1998). The HA fusion peptide is well conserved among all HAs subtypes, and similarly to other viral fusion peptides, contains GxxG or GxxxG motifs, which are believed to be responsible for the interactions with membranes or transmembrane domains (Cross et al., 2009; Worch, 2014). Among the fusion peptide amino acid sequence, many hydrophobic amino acids can be found, with acidic residues at the conserved positions 11, 15 and 19, which may contribute to the insertion of the fusion peptide into the target cell membrane (Cross et al., 2009; Worch, 2014), as describe below.

The FLUAV HA has two key functions: i) to bind to the major viral receptor determinant, sialic acid attached to proteins or lipids on the cell membrane, and ii) to fuse the viral membrane with a target cell membrane. The HA proteins of influenza viruses exhibit differential receptor specificity: Human and swine influenza viruses bind to $\alpha$-2,6-linked sialic acid, while avian and equine influenza viruses prefer sialic acid attached to galactose by a-2,3-linkeage (de Graaf and Fouchier, 2014; Matrosovich et al., 1993; Suzuki et al., 2000). The receptor preference correlates with the availability of appropriately linked sialic acids on cells of the respiratory tract: The majority of sialic acid isomers in the upper respiratory tract of humans is attached to the galactose by $\alpha-2,6$-linkage, while in the lower respiratory tract the $\alpha$-2,3-sialic acid isomers are predominant (Matrosovich et al., 2009; Suzuki et al., 2000). 
In case of swine, $\alpha-2,6$-linked sialic acids are the predominant form in both upper and lower respiratory tract, while $\alpha-2,3$-linked sialic acids are found in small amounts in bronchioles and alveoli (Trebbien et al., 2011). The avian respiratory tract contains both $\alpha-2,3-$ and $\alpha-2,6-$ linked sialic acid (Trebbien et al., 2011) However, in the intestinal tract, a target of FLUAV infection in birds but not mammals, $\alpha$-2,3-linked sialic acids predominate (Kumlin et al., 2008). The binding site for $\alpha-2,6$-linked sialic acid in HA of the H1 subtype is localized on the distal tip of $\mathrm{HA}_{1}$ subunit (Fig. 4, marked with star) and is formed by three secondary structures: one helix (190-helix, residues 190-198) and two loops (130-loop, residues 135138; and 220-loop, residues 221-228) (Chen et al., 1998; Sriwilaijaroen and Suzuki, 2012) (Mair et al., 2014; Wilks et al., 2012).

The second major HA function, mediation of viral entry, is described in the two following paragraphs.

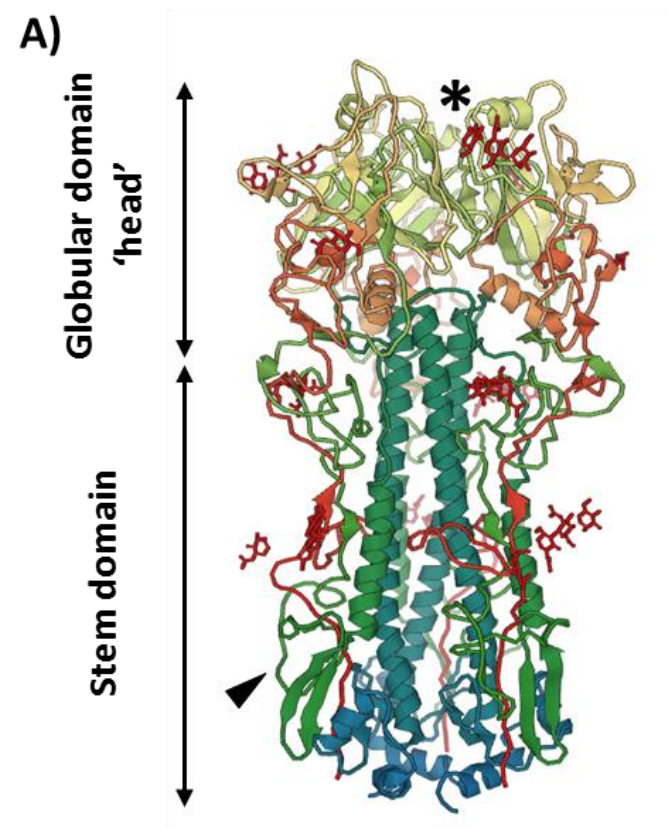

B)

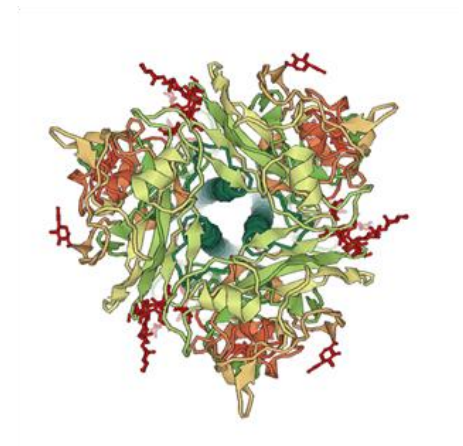

Figure 4. The structure of influenza A virus hemagglutinin. Structure of trimers of H1 subtype precursor $\mathrm{HA}_{0}$ in the side face-centered (A) or back $\mathrm{C} 3$ axis (B) orientation, with carbohydrates shown in red. The receptor binding site (star) and the cleavage site (arrowhead) are indicated. The figure was generated using Protein Data Bank (http://www.rcsb.org/pdb/home/home.do).

\subsubsection{Class I fusion proteins and membrane fusion mechanism}

The influenza virus HA exhibits a structural and functional organization also found in other viral glycoproteins, for instance those of paramyxoviruses, retroviruses, filoviruses, and coronaviruses, which are collectively termed class I membrane fusion proteins (Kielian, 
2014; White et al., 2008). Class I membrane fusion proteins are synthesized as single-chain precursors and assemble into trimers in the pre-fusion and post-fusion state. Upon successful execution of membrane fusion they form a characteristic six-helix-bundle structure (also called trimer of hairpins), consisting of a central $\mathrm{N}$-terminal trimeric $\alpha$-helical coiled-coil decorated by three C-terminal helices (Colman and Lawrence, 2003; Harrison, 2015; White et al., 2008). The single-chain precursor needs to be proteolytically processed to gain its fusogenic activity. This process occurs late in the biosynthetic pathway, as the fusion proteins, once cleaved, are in a metastable state and rapidly transit into an inactive, highly stable conformation (Colman and Lawrence, 2003; Harrison, 2015). In contrast to class II membrane fusion proteins, influenza HA and other viral class I membrane fusion proteins are characterized by a perpendicular orientation with respect to the viral membrane. Moreover, the predominating structural element in class I membrane fusion proteins are $\alpha$-helixes, while $\beta$-sheets are characteristic for class II membrane fusion proteins and both $\alpha$-helixes and $\beta$ sheets are found in class III membrane fusion proteins (White et al., 2008). In contrast, all classes of membrane fusion proteins transit into the six-helix-bundle structure after successful completion of membrane fusion (Colman and Lawrence, 2003; Harrison, 2015; Kielian, 2014; White et al., 2008).

Host cell entry driven by HA commences with binding of HA to appropriately linked sialic acids, followed by endocytic uptake of virions, as described in paragraph 3.4. At this stage, the fusion peptide of HA is hidden within the molecule (Figure 5). As endosomes become acidified, low $\mathrm{pH}$ triggers a series of conformational changes in HA. First, the $\mathrm{HA}_{1}$ subunit dissociates from the $\mathrm{HA}_{2}$ subunit although both remain linked by a disulfide bond (Harrison, 2015; Kielian, 2014). The dissociation of $\mathrm{HA}_{1}$ allows the N-terminal fusion peptide in $\mathrm{HA}_{2}$ to leave its buried position and to transit to the tip of the $\mathrm{HA}_{2}$ trimer (Cross et al., 2009; Harrison, 2015; Worch, 2014). The loop between the shorter and longer helices in $\mathrm{HA}_{2}$ becomes a helix (so called loop-to-helix transition), which creates a long, three-chain coiled coil. In the course of these transitions the fusion peptide is projected towards and ultimately inserts into the target membrane (Figure 5) (Cross et al., 2009; Worch, 2014). Next, the amino acids at $\mathrm{HA}_{2}$ positions 106-112 undergo a helix-to-loop transition, which causes the collapse of the extended intermediate and generates the post-fusion conformation, in which the fusion peptides are located in close proximity to the transmembrane domains. As a consequence of these transitions, target and viral membranes are pulled together and fuse (Figure 5) (Harrison, 2015). Membrane fusion commences with a hemifusion intermediate 
(Worch, 2014), a condition in which the outer leaflets of two bilayers are combined and the inner leaflets remain intact, followed by formation of a fusion pore (Figure 5).

1)

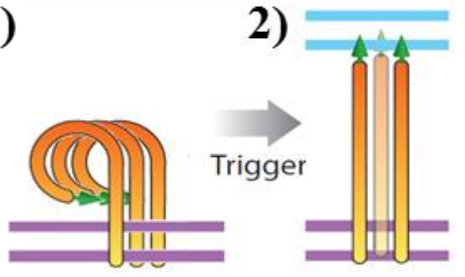

3)

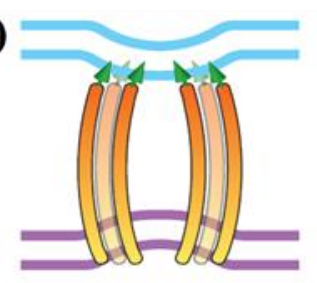

4)

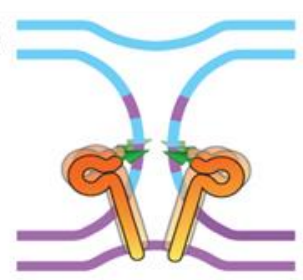

5)

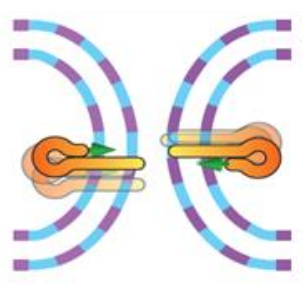

Figure 5. Scheme of membrane fusion driven by class I membrane fusion proteins. (1) The fusion peptide (green arrowhead), located in the transmembrane subunit, is hidden within the molecule. (2) A trigger, frequently low $\mathrm{pH}$, induces conformational changes in the fusion protein, in the course of which an extended intermediate is formed and the fusion peptide is inserted into the target cell membrane. At this stage, the fusion protein is connected to the viral (purple) and cellular (blue) membranes. (3) Fusion peptide insertion into the target cell membrane and conformational changes in the fusion protein alter the viral and the target cell membrane curvature and pull them into close contact. (4) Subsequently, the transmembrane unit refolds into a trimer-of-hairpins structure, resulting in the formation of a hemifusion intermediate, in which viral and host cell membranes are mixed. (5) The completion of the conformational changes allows the opening of the fusion pore and thus the finalization of the membrane fusion reaction (Figure was modified from Kielian, 2014).

\subsubsection{Proteolytic cleavage as a requirement of membrane fusion}

The HA-driven fusion of the viral and the endosomal membrane allows the release of the viral genetic material into the host cell cytoplasm and is essential for viral replication. HA is synthesized as an inactivate precursor and gains its membrane fusion activity only upon cleavage by a host cell protease (Lazarowitz et al., 1973a), which liberates the fusion peptide from the $\mathrm{HA}_{1}$ subunit, as discussed above. The $\mathrm{HA}_{0}$ precursor is cleaved at a linker sequence in a loop located between the $\mathrm{HA}_{1}$ and $\mathrm{HA}_{2}$ subunits (Fig. 6). First, $\mathrm{HA}$ is cleaved by endoproteases at the carboxyl terminus of an arginine located at the loop linking the $\mathrm{HA}_{1}$ and $\mathrm{HA}_{2}$ subunits and as a result the $\mathrm{N}$-terminus of $\mathrm{HA}_{2}$ is generated. Subsequently, the C-terminal arginine of the $\mathrm{HA}_{1}$ subunit is removed by an unknown cellular carboxypeptidase (Garten and Klenk, 1983). Whether this step is critical for HA function remains unclear (Hamilton et al., 2012b). The major structural consequence of proteolytic cleavage is the generation of a new C-terminus of $\mathrm{HA}_{1}$ subunit and a new $\mathrm{N}$-terminus of the $\mathrm{HA}_{2}$ subunit as well as the relocation of N-terminal fusion peptide (Cross et al., 2009). 
FLUAV can contain different amino acid sequence at the cleavage site. The $\mathrm{HA}_{0}$ with series of basic (multibasic) amino acids $\mathrm{R}-\mathrm{X}-\mathrm{R} / \mathrm{K}-\mathrm{R} \downarrow \mathrm{G}-\mathrm{L}-\mathrm{F}$ or $\mathrm{R}-\mathrm{X}-\mathrm{X}-\mathrm{R} \downarrow \mathrm{G}-\mathrm{L}-\mathrm{F}$, where $\mathrm{X}$ is a non-basic amino acid at the proteolytic cleavage site and $\downarrow$ indicates cleavage site, is found mainly in HPAIV, i.e. H5 and H7 avian influenza viruses (Garten et al., 2015; Hamilton et al., 2012b; Stieneke-Grober et al., 1992). In contrast, LPAIV as well as human influenza viruses contain P3-P3' sequence motif $\mathrm{Q} / \mathrm{E}-\mathrm{S} / \mathrm{T}-\mathrm{R} \downarrow \mathrm{G}-\mathrm{L}-\mathrm{F}$, with a single arginine at the cleavage site, a so called mono-basic cleavage site (Garten et al., 2015). Depending on the sequence of the cleavage site, different proteases are involved in the proteolytic cleavage and this has important consequences for spread and pathogenesis of avian influenza viruses (Hamilton et al., 2012b). The poly-basic cleavage site of HPAIV is processed intracellularly in the trans-Golgi compartment by ubiquitously expressed proteases, furin and other proprotein convertases (Stieneke-Grober et al., 1992). Due to the expression of these proteases in a wide range of cells and tissues, HPAIV can spread systemically and frequently cause fatal disease. In contrast, it is thought that expression of proteases activating LPAIV $\mathrm{HA}$ is restricted to the aerodigestive tract of these animals (Bertram et al., 2010b). As a consequence, viral spread is limited to these organs and can thus be controlled by the immune system. In addition, recent studies suggested that there are also factors, other than cleavage site, which play a role in HA activation. Kido et al. showed that the flanking regions of cleavage site play a role in HA cleavage by host cell proteases and Hamilton et al. suggested that the HA cleavage can be controlled by many other factors, since for most of the HA, the cleavage site amino acid sequence is identical (Hamilton et al., 2012b; Kido et al., 2012).

The HAs of human influenza viruses, including that of the highly pathogenic 1918 influenza virus, is monobasic and the nature of the host cell proteases responsible for activation of these HA proteins (as well as the HA proteins of LPAIV) is unclear. Several studies proposed that secreted protease expressed in the aerodigestive tract, including plasmin, tryptase Clara are responsible (Gotoh et al., 1990; Kido et al., 1992; Lazarowitz et al., 1973b; Murakami et al., 2001). However, many of these studies were conducted with recombinant proteins and the concentrations tested might not reflect those present in the relevant tissues of the infected host. Additionally, in case of the laboratory adapted FLUAV A/WSN/33, the NA was shown to sequester plasminogen, which upon conversion to plasmin, facilitates HA cleavage (Goto and Kawaoka, 1998). Similarly, the NA of the 1918 influenza virus can facilitate trypsin-independent activation of HA (Tumpey et al., 2005). However, 
this process is cell line-specific and plasminogen-independent (Chaipan et al., 2009), and the mechanism remains unclear.

A study by Zhirnov and colleagues showed that cleavage of influenza virus HA in human respiratory epithelium is a cell-associated process, accomplished by unknown serine proteases (Zhirnov et al., 2002). These results suggested that membrane-associated and not secreted proteases are responsible for activation of human influenza viruses and triggered efforts to identify the responsible enzymes. A study by Böttcher and colleagues showed that influenza viruses representing all subtypes pandemic in humans (H1N1, H2N2 and H3N2) are activated by TMPRSS2 and HAT (human airway trypsin-like protease), members of the type II transmembrane serine protease (TTSP) family, at least upon directed expression of these enzymes in a cell line (Böttcher et al., 2006). Subsequent studies showed confirmed activation of HA by TMPRSS2 and HAT, and showed that another TTSP, TMPRSS4, can also activate HA (Chaipan et al., 2009; Sawoo et al., 2014). These studies focused research interest on the role of TTSPs in viral infection, particularly on the question whether these enzymes can support viral spread in the infected host.

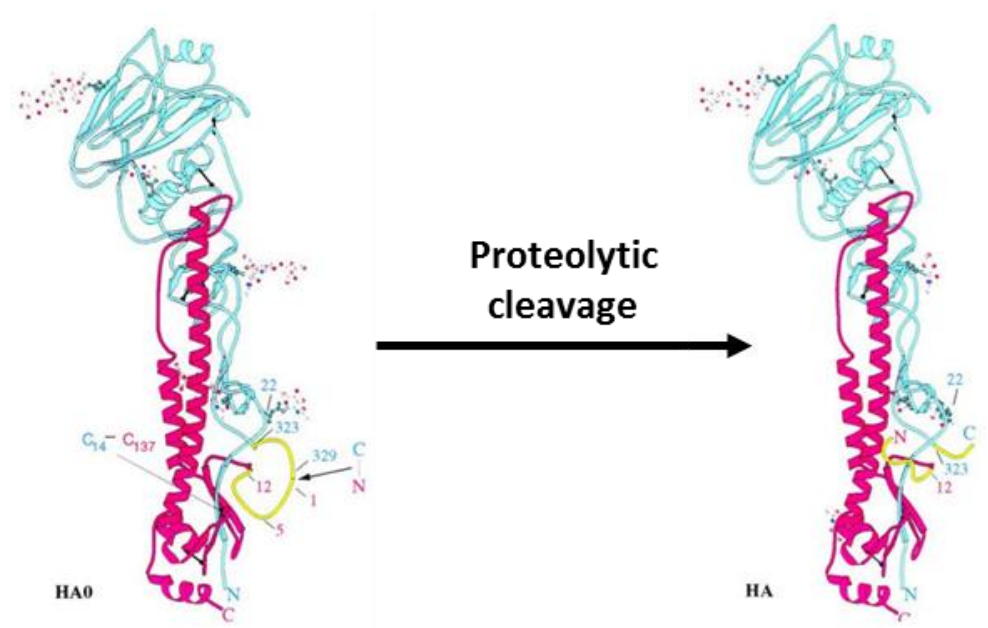

Figure 6. Proteolytic cleavage of influenza hemagglutinin. The single chain precursor of hemagglutinin, $\mathrm{HA}_{0}$, is cleaved into two subunits $\mathrm{HA}_{1}$ (blue) and $\mathrm{HA}_{2}$ (red) by host cell proteases. The cleavage site is located in a loop between $\mathrm{HA}_{1}$ and $\mathrm{HA}_{2}$ (yellow). After cleavage, residues 323 in $\mathrm{HA}_{1}$ and 1-12 in $\mathrm{HA}_{2}$ relocate, while residues 1-322 in $\mathrm{HA}_{1}$ and 13-137 in $\mathrm{HA}_{2}$ occupy the same position before and after proteolytic processing. Proteolytic cleavage liberates the fusion peptide in $\mathrm{HA}_{2}$ from $\mathrm{HA}_{1}$ but both subunits remain covalently linked by a single disulfide bond (Figure was modified from Chen et al., 1998). 


\subsection{Type II transmembrane serine proteases}

\subsubsection{Structure and function}

The type II transmembrane serine protease (TTSP) family is composed of more than 20 members and constitutes the largest group of membrane-anchored serine proteases. These proteins are synthesized as single-chain inactive precursors, zymogens, and display the following domain organization: The $\mathrm{N}$-terminus is located in the cytoplasm and is followed by a transmembrane domain, a stem domain and a catalytic serine protease domain (Fig. 7) (Antalis et al., 2011). The activation of TTSP zymogens requires cleavage after a basic amino acid residue (Arg or Lys) located in a conserved activation motif at the $\mathrm{N}$-terminus of the catalytic domain (Bugge et al., 2009). After cleavage, the catalytic domain remains associated with the remainder of the molecule due to a disulfide bond. However, this bond can be severed, for instance by mechanical stress, resulting in the release of the enzymatically active protease domain in the extracellular space (Szabo and Bugge, 2011). Several TTSPs, including matriptase, hepsin, TMPRSS2, TMPRSS3 and TMPRSS4 can undergo autocatalytic activation in vitro (Antalis et al., 2011). However, the mechanism of autoactivation is still unclear and probably involves oligomerization or spontaneous conformation-driven hydrolysis (Antalis et al., 2011; Bugge et al., 2009).

The TTSP domains have distinct functions in TTSP biology: The cytoplasmic tail and transmembrane domain target the proteases to the plasma membrane and localize their activities to specific sites, i.e. HAT on the basolateral and apical surfaces of polarized cells (Garten et al., 2015). In contrast, the stem region, which can contain up to 11 different modules with sequence similarity to cellular proteins, appears to facilitate interactions with cellular binding partners and to contribute to enzymatic activity of TTSPs (Antalis et al., 2011). Its presence is essential for the cleavage of certain physiological substrates (Bugge et al., 2009). Moreover, the stem region can contribute to the cellular localization, activation, inhibition, and/or substrate specificity of TTSPs (Antalis et al., 2011). The serine protease domain is responsible for the catalytic activity and contains a catalytic amino acid triad (histidine, aspartic acid and serine). It was shown that the catalytic domains of all TTSPs possess two adjacent, six-stranded $\beta$-barrel domains, connected via three linking transdomain segments (Bugge et al., 2009). The catalytic triad is located along the junction between two barrels and the active site cleft runs perpendicularly to this junction. Depending 
on the size, shape and charge distribution within this pocket, different substrates are recognized and processed.

The proteolytic processing of HA and other substrates by TTSPs depends on a twostep mechanism: i) an acylation step, in which an intermediate between enzyme and polypeptide is formed and a fragment of the polypeptide is lost, and ii) a deacylation step, in which water launches a nucleophilic attack on the intermediate, resulting in the hydrolysis of polypeptide (Antalis et al., 2011).

Four TTSP subfamilies have been defined according to phylogenetic analysis and similarities in domain structure: Hepsin/TMPRSS, Matriptase, Corin and HAT/DESC (Figure 7). The biggest subfamily, Hepsin/TMPRSS, contains seven members (enteropeptidase, hepsin, TMPRSS2, TMPRSS3, TMPRSS4, TMPRSS5 and MSPL). Except for enteropeptidase, all members of the Hepsin/TMPRSS subfamily contain a group A scavenger receptor domain in their stem regions. These proteases are predominantly expressed in fetal liver and kidney, prostate, as well as in the lung and parts of the gastrointestinal tract (Antalis et al., 2011; Uhlen et al., 2015). The HAT/DESC subfamily comprises HAT, DESC1, TMPRSS11A, TMPRSS11B and TMPRSS11F and these enzymes contain a single SEA (sea urchin sperm protein/enteropeptidase/agrin) domain in the stem (Antalis et al., 2011). The expression of the HAT/DESC subfamily members is more restricted in comparison to members of the Hepsin/TTSP family, and is limited to the upper respiratory tract (bronchus, trachea and nasal gland) (Antalis et al., 2011; Uhlen et al., 2015). The Matriptase subfamily contains four members, three highly homologous proteases (matriptase, matriptase- 2 and matriptase-3) and polyserase-1, which were found to be expressed in human breast cancer cell lines (matriptase)(Jin et al., 2005), liver (matriptase-2) (Uhlen et al., 2015), brain, ovary, testis and salivary gland (matriptase-3) (Szabo et al., 2005) as well as skeletal muscles, placenta and heart (polyserase-1) (Cal et al., 2003). The smallest TTSPs subfamily contains only one member, corin, which is mainly expressed in the heart (Chen et al., 2010).

TTSPs may play a role in development and homeostasis. For instance, matriptase is essential for hair follicle development (List et al., 2006) and matriptase-2 contributes to iron metabolism (Wang et al., 2014). A major function of TTSPs is the activation of precursor molecules. For instance, corin converts pro-atrial natriuretic peptide to atrial natriuretic factor, a cardiac hormone responsible for blood pressure regulation and cardiac function (Knappe et al., 2004). TTSPs can process a range of substrates, including peptide hormones, 
growth and differentiation factors, receptors, enzymes and adhesion molecules (Antalis et al., 2011). Due to the important biological functions of many TTSPs, changing their expression/structures/activities can results in several pathological conditions. It was demonstrated that single nucleotide polymorphisms in the corin gene, which alter the amino acid sequence of the frizzled domain located in the corin stem region, affects zymogen activation and activation of atrial natriuretic factor and is associated with hypertensive disease in humans (Knappe et al., 2004). TMPRSS3 and TMPRSS5 are expressed in inner ear tissues and mutations in these genes have been found in patients with familiar congenital deafness (Guipponi et al., 2008a; Guipponi et al., 2002). There is also growing evidence for the participation of TTSPs in the pathogenesis of inflammatory and neoplastic diseases. It is well established that TMPRSS2 participates in prostate cancer development. Thus, tmprss 2 mRNA levels are increased in androgen-dependent prostate cancer (Lucas et al., 2008), and tmprss 2 gene duplications were observed in cancer patients (Afar et al., 2001). Lucas et al. demonstrated also that the TMPRSS2 is expressed at high levels in primary and metastatic prostate cancers, and the TMPRSS2 cellular localization in most high-grade cancers was altered (Lucas et al., 2008). Additionally, it was demonstrated that the most frequent genomic alteration in prostate cancer is the fusion of TMPRSS2 with ERG (estrogen regulated gene) (Gasi Tandefelt et al., 2014). Due to the fact that TMPRSS2 is an androgen-responsive gene (Lucas et al., 2008), ERG overexpression as an effect of gene fusion can contribute to development of androgen-independence in prostate cancer by changes in the androgen receptor signaling (Hossain and Bostwick, 2013). Similarly, it is believed that dysregulated expression of matriptase promotes cancer development by processing the pro-forms of urokinase-type plasminogen activator and hepatocyte growth factor (Benaud et al., 2002), which augment invasive cellular growth. However, the current understanding of the impact of TTSP on many physiological and pathological processes is incomplete. 


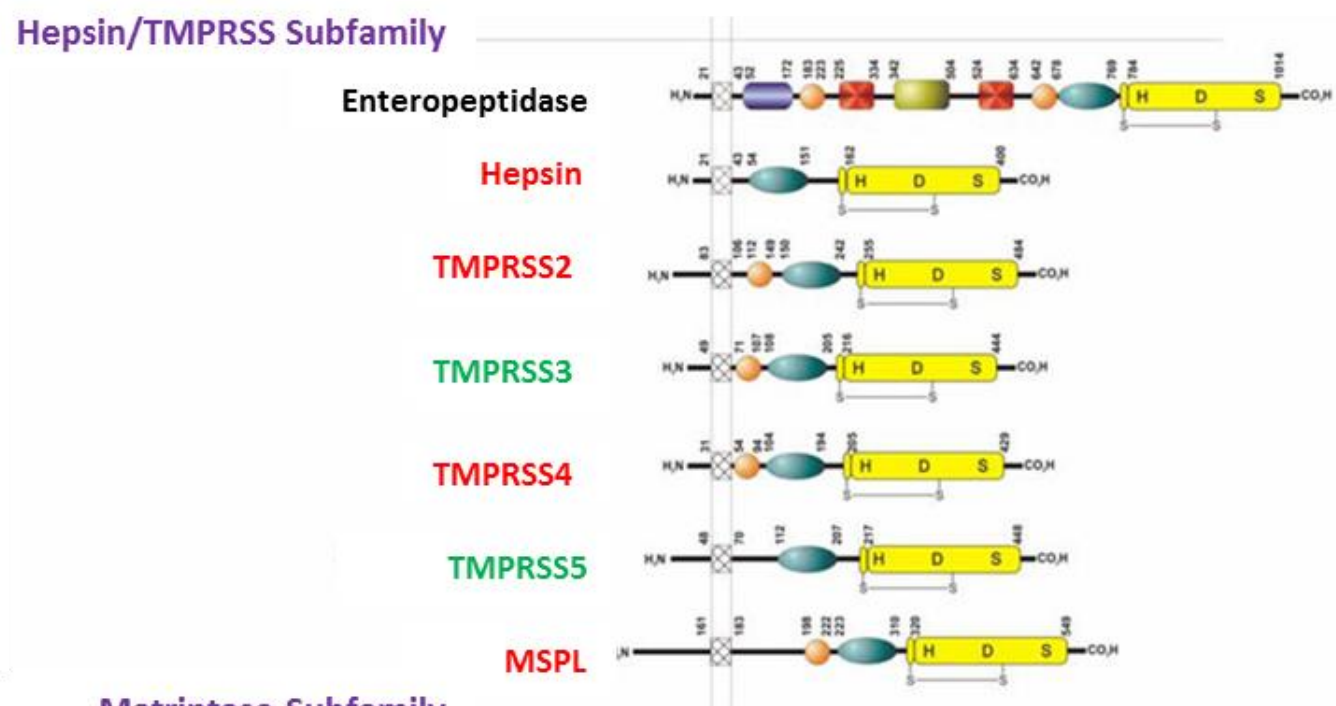

Matriptase Subfamily

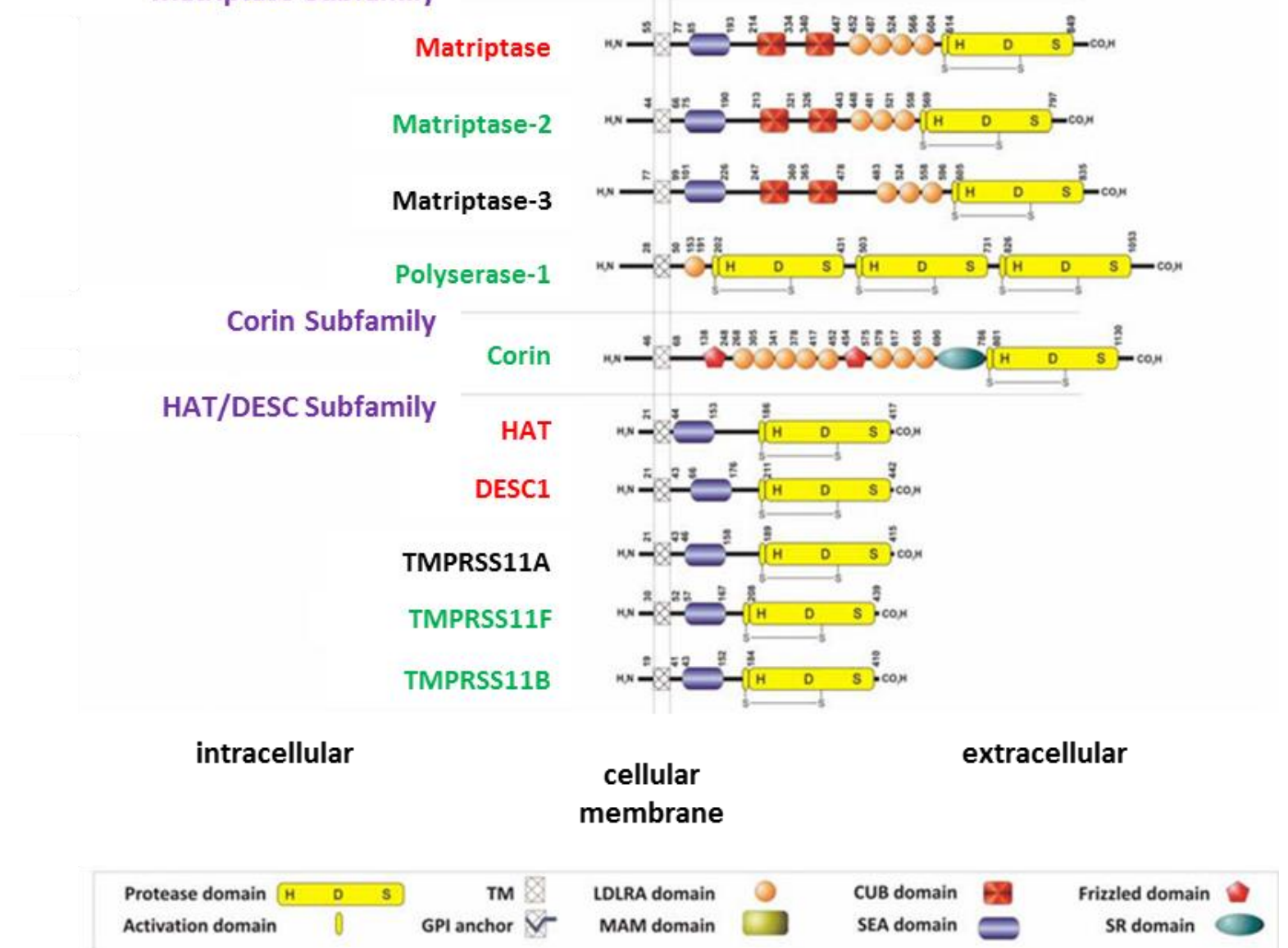

Figure 7. The type II transmembrane serine proteases. The enzymes are grouped according to similarities in domain structure and amino acid sequence homology. The first and last amino acid position in each domain is indicated above the domain. The HA-activating proteases are indicated in red, enzymes which fail to activate FLUAV are marked in green and enzymes not tested for HA activation are marked in black. Protease domain: serine protease domain; activation domain: prodomain; TM: transmembrane domain; GPI anchor: glycosyl-phosphatidylinositol linkage domain; LDLRA: LDL receptor class A domain; MAM: meprin, A5 antigen, and receptor protein phosphatase $\mu$ domain; CUB: Cls/Clr, urchin embryonic growth factor and bone morphogenetic protein-1; SEA: sea urchin sperm protein, enteropeptidase, agrin domain; Fz: frizzled domain; SR: Group A scavenger receptor domain (Figure was modified from Antalis et al., 2011). 


\subsubsection{Role of TMPRSS2 in FLUAV activation}

The observation that TMPRSS2 can activate HA upon directed expression in cell culture raised the question whether this protease might contribute to viral spread in the infected host. A series of studies indicate that this is indeed the case: It was demonstrated that endogenous expression of TMPRSS2 is responsible for trypsin-independent spread of FLUAV in the human cell lines Calu-3 (Böttcher-Friebertshäuser et al., 2011) and Caco-2 (Bertram et al., 2010a) and its was shown that TMPRSS2 is coexpressed with $\alpha-2,6$-linked sialic acid in human respiratory epithelia (Bertram et al., 2012). Notably, cleavage of HA by TMPRSS2 and HAT might proceed in different cellular compartments: HAT cleaves FLUAV $\mathrm{HA}$ at the cell surface and might proteolytically process HA of incoming viruses (BöttcherFriebertshäuser et al., 2010), although this is debated (Bertram et al., 2010b), as well as newly synthesized viral glycoprotein, while TMPRSS2 cleaves only newly synthesized HA within the infected cell (Böttcher-Friebertshäuser et al., 2010). Finally, Hatesuer and colleagues demonstrated that TMPRSS2 is essential for influenza H1N1 virus spread and pathogenesis in mice (Hatesuer et al., 2013). This study showed that the tmprss 2 knock-out mice were protected from viral spread and pathogenesis upon infection with H1N1 FLUAV, due to the lack of HA proteolytic processing (Hatesuer et al., 2013), a results that was confirmed by two subsequent studies (Sakai et al., 2014; Tarnow et al., 2014), which examined activation of $\mathrm{H} 1 \mathrm{~N} 1, \mathrm{H} 3 \mathrm{~N} 2$ and H7N9. In contrast, Hatesuer and colleagues demonstrated that tmprss2-deficient mice were not completely resistant to infection with H3N2 FLUAV (Hatesuer et al., 2013), suggesting that certain FLUAV might be able to hijack proteases other than TMPRSS2 for their spread in the host, and members of the TTSP family are attractive candidates. Indeed, several TTSPs other than TMPRSS2 can activate HA in cell culture but their potential role in FLUAV spread in the infected host is unknown.

The finding that TMPRSS2 can activate influenza HA raised the question whether this protease and related TTSP can also activate the glycoproteins of other viruses. Indeed, TMPRSS2 was found to cleave and activate the surface glycoprotein of coronaviruses, i.e. severe acute respiratory syndrome (SARS) coronavirus (Bertram et al., 2011; Kawase et al., 2012; Matsuyama et al., 2010; Shulla et al., 2011), Middle East respiratory syndrome (MERS) coronavirus (Gierer et al., 2013; Shirato et al., 2013), 229E coronavirus (Bertram et al., 2013), porcine epidemic diarrhea virus (PEDV) (Shirato et al., 2011), human metapneumovirus (Shirogane et al., 2008), human parainfluenza virus (Abe et al., 2013) and hepatitis C virus (Esumi et al., 2015). In sum, TMPRSS2 is hijacked by several viruses to 
promote their spread and inhibitors targeting this protease might thus exert broad antiviral activity. TMPRSS2 expression is dispensable for normal development and homeostasis in mice (Kim et al., 2006), suggesting that blockade of TMPRSS2 might not be associated with substantial unwanted side effects.

\subsubsection{Role of type II transmembrane serine proteases in influenza virus replication: Impact on steps other than $\mathrm{HA}$ activation}

Recent findings suggest that TMPRSS2 might impact viral spread at stages other than HA activation. Release of PEDV from infected cells was shown to be augmented by expression of TMPRSS2 and this effect was not observed in the presence of leupeptin, a broad spectrum protease inhibitor active against TMPRSS2 (Shirato et al., 2011). In the absence of active TMPRSS2 infected cells retained large clusters of virions on their surface and these structures were detached from cells upon trypsin treatment (Shirato et al., 2011). Collectively, these findings suggest that TMPRSS2 and potentially other TTSPs might promote release of certain viruses from infected cells, likely due to inactivation of a cellular factor which negatively regulates release of progeny virions. The interferon-induced antiviral host cell factor tetherin (CD317, BST-2) is such a factor: tetherin restricts replication of many enveloped viruses by blocking their release from the infected cells (Neil et al., 2008; Van Damme et al., 2008). The key to the tetherin's antiviral activity is its membrane topology: in opposite to TTSPs and HA, both $\mathrm{N}$ - and C- termini of tetherin are membrane anchored. This particular architecture allows the protein to insert one of its termini in the budding viral membrane, with the second one remaining in the host cell, thereby generating a physical bridge which results in virus trapping on the cell surface (Neil et al., 2008). Recently, it was also demonstrated that tetherin serves as a pattern recognition receptor, which induces NF- $\kappa \mathrm{B}-$ dependent proinflammatory gene expression in virus infected cells (Galao et al., 2012). Importantly, treatment of cells with trypsin abrogates tetherin's antiviral activity (Hammonds et al., 2010) raising the question whether endogenously expressed serine protease, in particular TTSPs, can also counteract tetherin. 


\section{Aims}

The influenza virus hemagglutinin (HA) is inserted into the viral envelope and mediates viral entry into the host cell. HA is synthesized as a single-chain precursor and depends on proteolytic activation by cellular enzymes to gain its ability to fuse membranes, which is a prerequisite to infectious viral entry. TMPRSS2, which belongs to the type II transmembrane serine protease (TTSP) family, was found to be essential for spread of influenza viruses of the H1N1 subtype in infected mice. Thus, TMPRSS2 is a potential target for antiviral intervention. However, an H3N2 virus was less dependent on TMPRSS2 and might hijack other TTSPs for spread in the host. Indeed, several members of the type II transmembrane serine protease (TTSP) family, i.e. TMPRSS2, TMPRSS4, HAT and matriptase, can activate HA in cell culture and potentially in the host. However, many TTSPs have not been examined for their ability to activate HA and the impact of alternative splicing of TTSP transcripts on HA activation by the encoded proteins are unknown. Moreover, it is currently unclear why only some TTSPs can activate HA, while other, closely related proteases cannot. Finally, it remains to be elucidated whether TTSPs might promote influenza virus infection by mechanisms independent of HA activation. Such a possibility is suggested by the observation that TMPRSS2 facilitates release of an animal coronavirus from infected cells, potentially by inactivating an antiviral cellular factor.

Therefore, four aims were defined for the present study:

i) to screen the TTSP family for new activators of human influenza viruses,

ii) to identify molecular determinants in TTSPs, which control the ability of these enzymes to cleave and activate HA,

iii) to test whether alternative splicing of TMPRSS2 alters HA cleavage,

iv) to test whether TMPRSS2 can promote influenza virus release, potentially by antagonizing antiviral cellular factors like tetherin (BST-2, CD317), which block viral release. 


\section{Manuscripts}

DESC1 and MSPL activate influenza A viruses and emerging coronaviruses for host cell entry Journal of Virology, 2014, 88: 12087-12097

The stem domain of type II transmembrane serine proteases determines the activation of viral glycoproteins and is a potential target for intervention Journal of Virology, submitted

TMPRSS2 isoforms 1 activates respiratory viruses and is expressed in viral target cells PLOS ONE, 2015, 10: $\mathrm{e} 0138380$

Tetherin sensitivity of influenza A viruses is strain specific: Role of hemagglutinin and neuraminidases Journal of Virology, 2015, 89: 9178-9188

Influenza virus activating host cell proteases antagonize tetherin Journal of Virology, submitted 


\title{
First manuscript
}

\section{DESC1 and MSPL activate influenza A viruses and emerging coronaviruses for host cell entry}

\author{
Zmora, P., Blazejewska, P., Moldenhauer, A.S., Welsch, K., Nehlmeier, I.,
} Wu, Q., Schneider, H., Pöhlmann, S., Bertram, S.

Journal of Virology, 2014, 88: 12087-12097; doi: 10.1128/JVI.01427-14

\section{Individual contribution:}

Analysis of the expression of type II transmembrane serine proteases by western blot (Fig. 1A) and flow cytometry (Fig. 1B). Analysis of the proteolytic cleavage of the coronavirus proteins (Fig. 2A and 2B). Determination whether the novel proteases cleave influenza virus hemagglutinin from different viral subtypes (Fig 3A, B, C). Microscopic analysis of the cellular localization of TTSPs and colocalization with influenza virus hemagglutinin (Fig. 6A) and calculation of the Person correlation coefficient (Fig. 6B). Production of lentiviral vectors bearing the influenza hemagglutin/neuraminidase and analysis of the HA activation in transduced cells (Fig. 7A). Propagation of influenza viruses in chorioallantoic fluid of embryonated hen eggs and analysis of the proteolytic activation of hemagglutinin by type II transmembrane serine proteases (Fig. 7B). Writing parts of the manuscript (Material and Methods, Results) 


\title{
DESC1 and MSPL Activate Influenza A Viruses and Emerging Coronaviruses for Host Cell Entry
}

\author{
Pawel Zmora, ${ }^{a}$ Paulina Blazejewska, ${ }^{a}$ Anna-Sophie Moldenhauer, ${ }^{a}$ Kathrin Welsch, ${ }^{\text {a*}}$ Inga Nehlmeier, ${ }^{\text {a }}$ Qingyu Wu, ${ }^{\text {b }}$ Heike Schneider, ${ }^{c}$ \\ Stefan Pöhlmann, ${ }^{\text {a }}$ Stephanie Bertram ${ }^{a *}$ \\ Infection Biology Unit, German Primate Center, Göttingen, Germanya; Molecular Cardiology, Nephrology, and Hypertension, Lerner Research Institute, Cleveland Clinic, \\ Cleveland, Ohio, USA ${ }^{\text {b }}$ Institute for Physiological Chemistry, Hannover Medical School, Hannover, Germany ${ }^{\mathrm{c}}$
}

\section{ABSTRACT}

The type II transmembrane serine protease (TTSP) TMPRSS2 cleaves and activates the influenza virus and coronavirus surface proteins. Expression of TMPRSS2 is essential for the spread and pathogenesis of H1N1 influenza viruses in mice. In contrast, H3N2 viruses are less dependent on TMPRSS2 for viral amplification, suggesting that these viruses might employ other TTSPs for their activation. Here, we analyzed TTSPs, reported to be expressed in the respiratory system, for the ability to activate influenza viruses and coronaviruses. We found that MSPL and, to a lesser degree, DESC1 are expressed in human lung tissue and cleave and activate the spike proteins of the Middle East respiratory syndrome and severe acute respiratory syndrome coronaviruses for cell-cell and virus-cell fusion. In addition, we show that these proteases support the spread of all influenza virus subtypes previously pandemic in humans. In sum, we identified two host cell proteases that could promote the amplification of influenza viruses and emerging coronaviruses in humans and might constitute targets for antiviral intervention.

\section{IMPORTANCE}

Activation of influenza viruses by host cell proteases is essential for viral infectivity and the enzymes responsible are potential targets for antiviral intervention. The present study demonstrates that two cellular serine proteases, DESC1 and MSPL, activate influenza viruses and emerging coronaviruses in cell culture and, because of their expression in human lung tissue, might promote viral spread in the infected host. Antiviral strategies aiming to prevent viral activation might thus need to encompass inhibitors targeting MSPL and DESC1.

\footnotetext{
nfluenza A viruses (FLUAVs) and the emerging pathogens severe acute respiratory syndrome coronavirus (SARS-CoV) and Middle East respiratory syndrome coronavirus (MERS-CoV) are respiratory viruses that pose a significant threat to human health. Annual influenza epidemics cause 250,000 to 500,000 deaths worldwide (1), and intermittent pandemics can have even more severe consequences, as exemplified by the devastating Spanish influenza of 1918, which is estimated to be responsible for 30 to 50 million deaths (2). The SARS-CoV emerged in southern China in 2002, and its subsequent spread, mainly in Asia, was responsible for 774 deaths (3). A related virus, the MERS-CoV, emerged in the Middle East in 2012 (4), and new cases of MERS-CoV infection continue to be reported in July 2014, with the total number of cases amounting to 834 , of which 288 took a fatal course (5). Therefore, the development of novel strategies to combat FLUAV and emerging CoVs is urgently required, and host cell factors essential for viral spread but dispensable for cellular survival are attractive targets.

The viral hemagglutinin (HA) and spike (S) surface proteins are responsible for host cell entry of FLUAV $(6)$ and $\mathrm{CoVs}(7,8)$, respectively. Both proteins use their $\mathrm{N}$-terminal surface units, termed HA1 (FLUAV) and S1 (CoVs), to engage cellular receptors, while the C-terminal transmembrane units, termed HA2 (FLUAV) and S2 (CoVs), facilitate fusion of the viral membrane with a host cell membrane-processes that are essential for infectious entry (6-8). Notably, the HA and S proteins are synthesized as inactive precursors in infected cells and acquire the ability to drive membrane fusion only upon activation by host cell proteases $(9,10)$. Activation refers to proteolytic separation of the surface and transmembrane units, which is essential for viral infectivity.
}

Consequently, the proteases responsible for $\mathrm{HA}$ and $\mathrm{S}$ protein activation are potential therapeutic targets.

It has been suggested that several proteases secreted in the lung lumen can activate FLUAV (11-13). However, examination of cultured human respiratory epithelium revealed a key role for membrane-associated proteases (14), and work by Böttcher and colleagues identified the transmembrane serine proteases TMPRSS2 and HAT as potent activators of FLUAV (15), at least upon engineered expression in cell lines. Subsequent studies showed that endogenous expression of TMPRSS2 in cell lines can support trypsin-independent FLUAV spread $(16,17)$, and coexpression of TMPRSS2 and 2,6-linked sialic acid has been demonstrated in most parts of the human airways (18), suggesting that TMPRSS2 might support FLUAV spread in the infected host. Indeed, work by Hatesuer and colleagues demonstrated that knockout of Tmprss 2 in mice largely abrogates the spread of FLUAV and

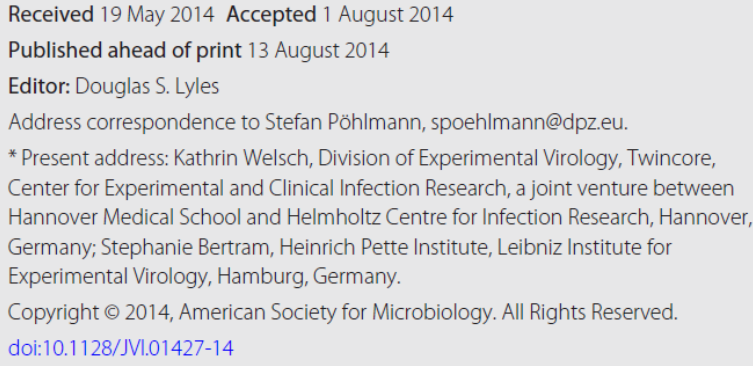


prevents viral pathogenesis (19) and similar findings were subsequently reported by other groups $(20,21)$. Moreover, TMPRSS2 was shown to activate the SARS- (22-24) and MERS- $(25,26)$ $\mathrm{CoV}$ s for entry into target cells in which the activity of cathepsin L, another protease able to activate SARS-CoV S (27) and MERSCoV S $(25,26)$, was blocked by inhibitors. Thus, FLUAV, highly pathogenic $\mathrm{CoVs}$, and several other respiratory viruses (28-30) can be activated by TMPRSS2.

An essential role for TMPRSS2 in FLUAV spread and pathogenesis in mice has been demonstrated with viruses of the H1N1 and H7N9 subtypes (19-21). Dependence on TMPRSS2 was also reported for a virus of the H3N2 subtype (20). In contrast, a different $\mathrm{H} 3 \mathrm{~N} 2$ virus tested in a separate study was much less dependent on TMPRSS2 expression (19), suggesting that certain H3N2 viruses might be able to usurp another protease to ensure their activation, and members of the type II transmembrane serine protease (TTSP) family, which comprises more than 20 proteins (31), are interesting candidates. Here, we assessed seven TTSPs previously reported to be expressed in the lung for the ability to activate the surface proteins of FLUAV, MERS-CoV, and SARS-CoV. We show that MSPL and, to a lesser degree, DESC1 are expressed in the human lung and activate FLUAV and CoV surface glycoproteins, suggesting that these proteases could contribute to viral spread in the host.

\section{MATERIALS AND METHODS}

Plasmid construction. Expression plasmids for SARS-CoV S (32), MERS-CoV S (25), FLUAV H1 HA $(15,33)$, ACE2 (34), and CD26 (DPP4) (25) have been described previously. Plasmids for $\mathrm{H} 2$ and $\mathrm{H} 3$ (15 HA of FLUAV were kindly provided by Mikhail Matrosovich, Institute of Virology, Marburg, Germany. Plasmids encoding the human transmembrane proteases TMPRSS2, HAT, TMPRSS3, TMPRSS9, and TMPRSS10 were also published earlier $(16,18,35-37)$. The sequences encoding human DESC1, MSPL, TMPRSS11F, prostasin, and TMPRSS11B were amplified by reverse transcription (RT)-PCR with mRNA prepared from Caco2 (DESC1, MSPL), H1299 (TMPRSS11F), or LNCaP (prostasin, TMPRSS11B) cells as the template. The protease sequences were inserted into plasmid pCAGGS by using the appropriate restriction sites. The resulting plasmids encode human TTSPs with amino acid sequences identical to those deposited in the GenBank database, i.e., DESC (AF064819.1), MSPL (NM_001077263.2), TMPRSS11F (NM_207407.2), prostasin (NM_002773.3), and TMPRSS11B (NM_182502.3). Expression plasmids for TTSPs with an N-terminal myc tag were generated by PCR by using the above-described plasmids as templates as reported previously (38). The integrity of all PCR-amplified sequences was verified by automated sequence analysis.

Cell culture. Human embryonal kidney 293T cells were propagated in Dulbecco's modified Eagle's medium (DMEM), and MDCK cells were grown in minimum essential medium. All media were supplemented with $10 \%$ fetal bovine serum, penicillin, and streptomycin. The cells were maintained in a humidified atmosphere containing $5 \% \mathrm{CO}_{2}$

Analysis of TTSP expression. For analysis of TTSP expression by Western blotting, 293T cells were seeded into six-well plates at a density o $2.2 \times 10^{5} /$ well, cultivated for $24 \mathrm{~h}$, and then transfected with plasmid encoding proteases equipped with an N-terminal myc tag or transfected with an empty plasmid as a control. At $16 \mathrm{~h}$ posttransfection, the medium was replaced with fresh DMEM, and at $48 \mathrm{~h}$ posttransfection, the cells were washed with phosphate-buffered saline (PBS) and detached with 100 $\mu$ l of $2 \times$ sodium dodecyl sulfate (SDS) loading buffer per well. All samples were denatured for $30 \mathrm{~min}$ at $95^{\circ} \mathrm{C}$, separated by SDS-PAGE, and blotted onto a nitrocellulose membrane (Hartenstein). The proteins were detected by using a mouse anti-myc antibody (Biomol) as the primary antibody and a horseradish peroxidase (HRP)-coupled anti-mouse anti- body (Dianova) as the secondary antibody. For analysis of TTSP expression by flow cytometry, 293T cells transfected with TTSP-encoding plasmids were detached, washed with PBS, incubated with ice-cold ethanol for $10 \mathrm{~min}$, and then stained with a mouse anti-myc antibody (Biomol) diluted in $0.1 \%$ saponin. Mouse IgG1 (R\&D Systems) was used as an isotype-matched control. After $30 \mathrm{~min}$ of incubation with primary antibodies at $4^{\circ} \mathrm{C}$, cells were washed twice with PBS and incubated for $30 \mathrm{~min}$ at $4^{\circ} \mathrm{C}$ with DyLight 647-coupled anti-mouse secondary antibodies (Dianova) diluted in $0.1 \%$ saponin. After two final washing steps, cells were fixed with $2 \%$ paraformaldehyde and staining was analyzed with an LSR II flow cytometer (BD Biosciences).

TaqMan-based quantitative RT-PCR analysis of protease expression in the lung. Two pools of commercially available RNA (Agilent Technologies), each prepared from normal human lung tissue obtained from four male donors, were used to quantify protease transcripts. Total RNA $(1 \mu \mathrm{g})$ was reverse transcribed with $50 \mathrm{U}$ of BioScript RNase H Low reverse transcriptase (BIO-27036; Bioline) in 20- $\mu$ l reaction mixtures. The enzyme was finally inactivated for $10 \mathrm{~min}$ at $70^{\circ} \mathrm{C}$. cDNA aliquots ( 1 $\mu \mathrm{l}$ ) corresponding to $50 \mathrm{ng}$ total RNA equivalents were used for real-time PCR in triplicate $10-\mu l$ reaction mixtures with the ABI 7500 Fast real-time PCR system (Applied Biosystems). Specific amplification was ensured by using TaqMan gene expression assays according to the manufacturer's recommendations. The specific assays used were Hs00237175_ml (TMPRSS2), Hs01070171_m1 (DESC1 = TMPRSS11E), Hs00361060_ml $($ MSPL $=$ TMPRSS13), and Hs99999908_m1 (GUSB). The average cycle threshold $\left(C_{T}\right)$ for each individual assay was calculated from triplicate measurements by means of the instrument's software in "auto $C_{T}$ " mode (ABI 7500 Fast system software, version 1.3.0). Average $C_{T}$ values calculated for TMPRSS2, DESC1, and MSPL were normalized by subtraction from the $C_{T}$ values obtained for GUSB (housekeeping reference). Template-free cDNA reaction mixtures prepared in triplicate were analyzed in parallel with all of the TaqMan assays, and no specific signal was detected in any of these negative controls.

Cleavage of SARS-CoV S, MERS-CoV S, and influenza virus HA by TTSPs. For the detection of SARS-CoV S, MERS-CoV S, and FLUAV HA cleavage by TTSPs, 293 T cells were seeded into six-well plates at a density of $2.2 \times 10^{5}$ /well and cotransfected with an expression plasmid for SARSCoV S with a C-terminal V5 tag, MERS-CoV S with a C-terminal V5 tag, or FLUAV HA of the $\mathrm{H} 1, \mathrm{H} 2$, or H3 subtype and either plasmids encoding the specified proteases or an empty plasmid. The medium was replaced with fresh DMEM at 8 to $16 \mathrm{~h}$ posttransfection. At $48 \mathrm{~h}$ posttransfection, the cells were harvested in $1 \mathrm{ml}$ of PBS, treated with PBS or tosylsulfony phenylalanyl chloromethyl ketone (TPCK)-trypsin (Sigma) (SARS-CoV S-positive cells were treated with $100 \mu \mathrm{g} / \mathrm{ml}$ TPCK-trypsin, while MERSCoV S- and HA-expressing cells were treated with $250 \mu \mathrm{g} / \mathrm{ml}$ TPCKtrypsin) for $10 \mathrm{~min}$ at room temperature and lysed in SDS loading buffer. The lysates were separated by SDS-PAGE and blotted onto nitrocellulose membranes. The SARS- and MERS-CoV S proteins with a C-terminal V5 antigenic tag $(25,32)$ were detected by staining with mouse monoclonal antibody specific for the V5 tag (Invitrogen), followed by incubation with an HRP-coupled anti-mouse secondary antibody (Dianova), respectively. The expression of FLUAV HA of the H1 subtype was detected by staining with a mouse monoclonal antibody reactive against the HA of the 1918 H1N1 FLUAV (39), followed by incubation with an HRP-coupled antimouse secondary antibody (Dianova). The expression of FLUAV HA of the $\mathrm{H} 2$ subtype was detected with a goat anti-FLUAV polyclonal antibody (Millipore) and an HRP-coupled anti-goat antibody (Dianova). For detection of expression of FLUAV HA of the $\mathrm{H} 3$ subtype, a rabbit anti-H3 HA serum (Immune Technology) and an HRP-coupled anti-rabbit antibody were used. As a loading control, expression of $\beta$-actin was detected with an anti- $\beta$-actin antibody (Sigma). Staining was detected with the ECL Prime Western blotting detection reagent (Amersham) as specified by the manufacturer and with a ChemoCam Imager (Intas). 
Analysis of proteolytic activation of the SARS- and MERS-CoV S proteins for cell-cell fusion. For analysis of SARS- and MERS-CoV S activation for cell-cell fusion, a previously described cell-cell fusion assay was used (40). In brief, 293T effector cells, seeded into six-well plates at 1.3 $\times 10^{5} /$ well, were calcium phosphate transfected with either the empty pCAGGS plasmid or pCAGGS encoding SARS- or MERS-CoV S in combination with plasmid pGAL4-VP16, which encodes the herpes simplex virus VP16 transactivator fused to the DNA binding domain of the yeast transcription factor GAL4. In parallel, 293T target cells were seeded into 48-well plates at $0.8 \times 10^{5} /$ well and transfected with the empty pcDNA3 plasmid or the expression plasmids for ACE2 or CD26 jointly with protease expression plasmids and plasmid pGal5-luc, which carries the firefly luciferase reporter gene under the control of a promoter containing five GAL4 binding sites. The day after transfection, effector cells were detached, diluted in fresh medium, and added to the target cells. For trypsin treatment, medium from target cells was completely removed and effector cells in medium supplemented with $100 \mathrm{ng} / \mathrm{ml}$ TPCK-treated trypsin (Sigma) or PBS were added. After $6 \mathrm{~h}$ of incubation time, the culture media were replaced with fresh medium without trypsin. Cell-cell fusion was quantified by determination of luciferase activities in cell lysates at 48 $\mathrm{h}$ after cocultivation with a commercially available kit (Promega).

Production of lentiviral pseudotypes and transduction experiments. The generation of lentiviral vectors was carried out as described previously $(16,25,41)$. In brief, $293 \mathrm{~T}$ cells were transiently cotransfected with pNL4-3-LucR ${ }^{-} \mathrm{E}^{-}$(42) and expression plasmids for SARS-CoV S, MERS-CoV S, HA and neuraminidase (NA) of the 1918 H1N1 FLUAV, or vesicular stomatitis virus glycoprotein (VSV-G) or the empty plasmid pCAGGS. For analysis of HA activation by TTSPs, the proteases analyzed were coexpressed with the viral components specified above during the generation of lentiviral pseudotypes. The culture medium was replaced at $16 \mathrm{~h}$ posttransfection, and supernatants were harvested at $48 \mathrm{~h}$ posttransfection. The supernatants were passed through $0.45-\mu \mathrm{m}$ filters, aliquoted, and stored at $-80^{\circ} \mathrm{C}$. For analysis of MERS- and SARS-CoV S-mediated transduction, 293T cells were cotransfected with expression plasmids for ACE2 or CD26 and the TTSPs indicated or an empty plasmid. The culture medium was replaced with fresh medium at $8 \mathrm{~h}$ posttransfection, and the cells were seeded into 96-well plates at $24 \mathrm{~h}$ posttransfection. At $48 \mathrm{~h}$ posttransfection, cells were preincubated with dimethyl sulfoxide (DMSO) or $10 \mu \mathrm{M}$ cathepsin inhibitor MDL 28170 (Calbiochem) for $1 \mathrm{~h}$ and then incubated with equal volumes of infectivity-normalized pseudotypes for $8 \mathrm{~h}$. Thereafter, the medium was changed and the luciferase activities in cell lysates were determined with a commercially available kit (Promega, Madison, WI) at $72 \mathrm{~h}$ postinfection. For the analysis of HAmediated transduction, $293 \mathrm{~T}$ cells were either directly seeded into 96-wel plates at a density of $0.8 \times 10^{4} /$ well or first transfected with proteaseencoding plasmids and then seeded into 96-well plates. Subsequently, the cells were incubated with equal volumes of infectivity-normalized vector preparations pretreated with PBS or TPCK-trypsin. At $12 \mathrm{~h}$ posttransduction, fresh medium was added, and at $72 \mathrm{~h}$ posttransduction, the cells were lysed and the luciferase activities in cell lysates were determined.

Production of influenza viruses and infection experiments. $\mathrm{A} / \mathrm{PR} /$ 8/34 (H1N1) (19) and A/Panama/2007/99 (H3N2) (43) were propagated in the chorioallantoic cavities of 10-day-old embryonated hen eggs (Harlan Winkelman, Germany) for $48 \mathrm{~h}$ at $37^{\circ} \mathrm{C}$. A/Panama/2007/99 (H3N2) was reconstituted from an eight-plasmid system (43) before amplification in eggs. For infection experiments, 293T cells were seeded into six-well plates at a density of $2.8 \times 10^{5} /$ well. After $24 \mathrm{~h}$, the cells were transfected with $6 \mu \mathrm{g}$ of expression plasmids encoding the indicated TTSPs or an empty plasmid by the calcium phosphate transfection method. The transfection medium was replaced with fresh medium after overnight incubation. At $24 \mathrm{~h}$ posttransfection, the culture medium was removed and the cells were incubated with infection medium (DMEM supplemented with $0.2 \%$ bovine serum albumin $[\mathrm{BSA}])$ containing $\mathrm{A} / \mathrm{PR} / 8 / 34(\mathrm{H} 1 \mathrm{~N} 1)$ at a multiplicity of infection (MOI) of 0.01 or A/Panama/2007/99 (H3N2) at an MOI of 0.1 . After $1 \mathrm{~h}$ of incubation at $37^{\circ} \mathrm{C}$, the infection medium was removed, the cells were gently washed with PBS, and fresh infection medium (again without trypsin) was added. Culture supernatants were collected at $48 \mathrm{~h}$ postinfection. The amount of infectious units within the culture supernatants was determined by focus formation assay as described previously by us (44). In brief, serial 10-fold dilutions of samples were prepared in infection medium (DMEM with $1 \%$ penicillin-streptomycin and $0.1 \%$ BSA) and added to MDCK cells. After $1 \mathrm{~h}$ of incubation, the medium was replaced with infection medium containing an Avicel overlay and $2.5 \mu \mathrm{g} / \mathrm{ml} \mathrm{N}$-acetylated trypsin (Sigma), and the cells were incubated for $24 \mathrm{~h}$. Subsequently, the cells were fixed with $4 \%$ formalin in PBS and incubated for $1 \mathrm{~h}$ with a goat anti-influenza virus nucleocapsid NP polyclonal antibody (Virostat), followed by $1 \mathrm{~h}$ of incubation with HRP-conjugated anti-goat antibodies (Dianova) and $10 \mathrm{~min}$ incubation with True Blue substrate (KPL). Foci were counted, and viral titers were calculated as numbers of focus-forming units per milliliter of culture supernatant.

Analysis of HA and TTSP colocalization. To determine the cellular localization of FLUAV HA and TTSPs, we seeded COS-7 cells onto coverslips in six-well plates at a density of $0.6 \times 10^{5} /$ well. The cells were calcium phosphate transfected with plasmids encoding TMPRSS2, TMPRSS3, DESC1, and MSPL, all containing an N-terminal myc antigenic tag. Cells transfected with an empty plasmid served as a negative control. At $16 \mathrm{~h}$ posttransfection, the medium was replaced with fresh DMEM, and at $24 \mathrm{~h}$ posttransfection, the cells were washed with PBS and infected with FLUAV A/PR/8/34 at an MOI of 1 . At $1 \mathrm{~h}$ postinfection, the medium was replaced with DMEM supplemented with $1 \mu \mathrm{g} / \mathrm{ml}$ TPCKtrypsin. At $24 \mathrm{~h}$ postinfection, the cells were treated with ice-cold methanol $\left(7.5 \mathrm{~min}\right.$ at $\left.4^{\circ} \mathrm{C}\right)$, blocked with $3 \%$ BSA for $1 \mathrm{~h}$, and then incubated with mouse anti-myc and rabbit anti-PR8 HA antibodies (Biomol and Sino Biological Inc., respectively). After $1 \mathrm{~h}$ of incubation with primary antibodies at room temperature, cells were washed three times with PBS and incubated for $1 \mathrm{~h}$ at room temperature with Rhodamine Red-X-coupled anti-mouse and fluorescein isothiocyanate (FITC)-coupled anti-rabbit secondary antibodies (Dianova). After three final washing steps, cells were treated with Vectashield 4',6-diamidino-2-phenylindole mounting medium (Vector Laboratories) and analyzed with a Zeiss LSM 5 laser scanning microscope. Image capture was performed with Pascal Software (Zeiss), and further image analysis, including calculation of the Pearson correlation coefficient (PCC), was done with ImageJ with Just Another Colocalization Plugin (45).

\section{RESULTS}

TTSPs are expressed in transfected $293 \mathrm{~T}$ cells. For all known human TTSPs, expression in the lung has been reported but expression levels differ profoundly (31) and for many of these enzymes it is unknown if they can cleave and activate viral glycoproteins. In order to address this question, we first amplified the sequences of seven TTSPs not previously characterized in the context of virus infection and then examined the expression of the cloned protease sequences in transiently transfected $293 \mathrm{~T}$ cells. For this, the protease sequences were fused to an N-terminal myc tag and protease expression was detected by Western blotting (Fig. 1A). The expression of most proteases was readily detectable, with the expression of TMPRSS2 and MSPL being particularly prominent and the expression of HAT and TMPRSS11F being relatively weak. In contrast, signals obtained for prostasin-expressing cells were close to (data not shown) or within the background range. The molecular weights of the proteases studied roughly matched the predicted ones, and bands expected upon autocatalytic activation were seen for all of the proteases except TMPRSS9 (a faint signal was consistently observed for TMPRSS11B).

In order to obtain further information on TTSP expression, we also analyzed TTSP levels by fluorescence-activated cell sorting 


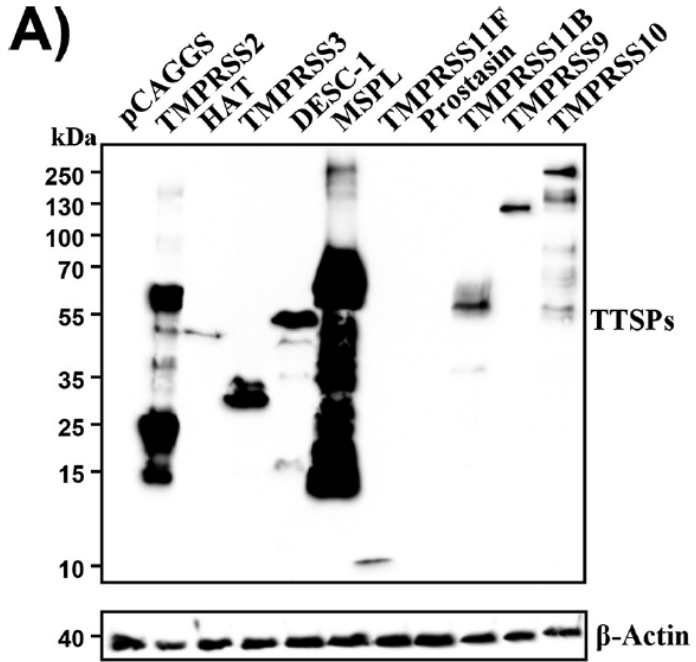

B)

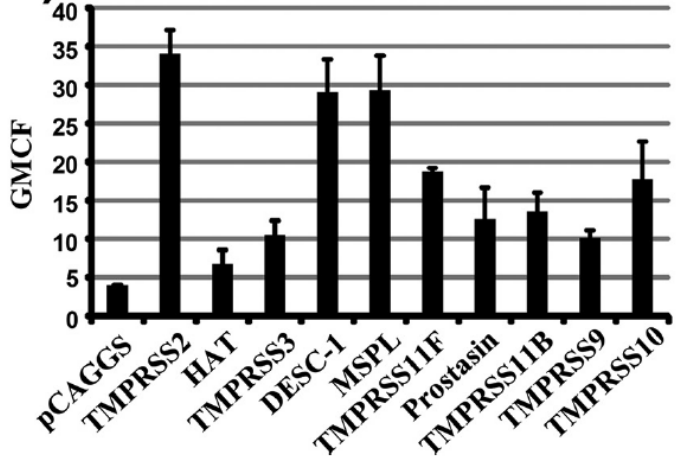

FIG 1 Expression of TTSPs in 293T cells. (A) Plasmids encoding the indicated proteases equipped with an N-terminal myc tag were transiently transfected into 293T cells. Transfection of an empty plasmid (pCAGGS) served as a negative control. Protease expression in cell lysates was detected by Western blotting with an anti-myc antibody. Detection of $\beta$-actin served as a loading control. Similar results were obtained in three separate experiments. (B) The experiment was conducted as described for panel A, but TTSP expression was detected by FACS. The geometric mean channel fluorescence (GMCF) measured in a representative experiment performed with triplicate samples is shown. Error bars indicate standard deviations. Similar results were obtained in three independent experiments.

(FACS) of stained permeabilized cells, which allows the detection of intracellular and surface-expressed TTSPs. Again, prominent signals were measured in TMPRSS2- and MSPL-expressing cells (Fig. 1B) and similar expression of DESC1 was noted. Specific and roughly comparable signals were measured in the cells expressing the remaining proteases, indicating that all of the proteases examined were produced in transiently transfected cells, although at different levels.

The TTSPs DESC1 and MSPL cleave the S proteins of the SARS- and MERS-CoVs. We first assessed if the TTSPs studied were able to cleave the $S$ proteins of the emerging SARS- and MERS-CoVs. Cleavage of MERS-CoV S was readily detected upon the coexpression of TMPRSS2 and HAT or upon the treatment of S-protein-expressing cells with trypsin (Fig. 2A), as expected (22$25,35)$. Proteolysis of MERS-CoV S was also observed upon the
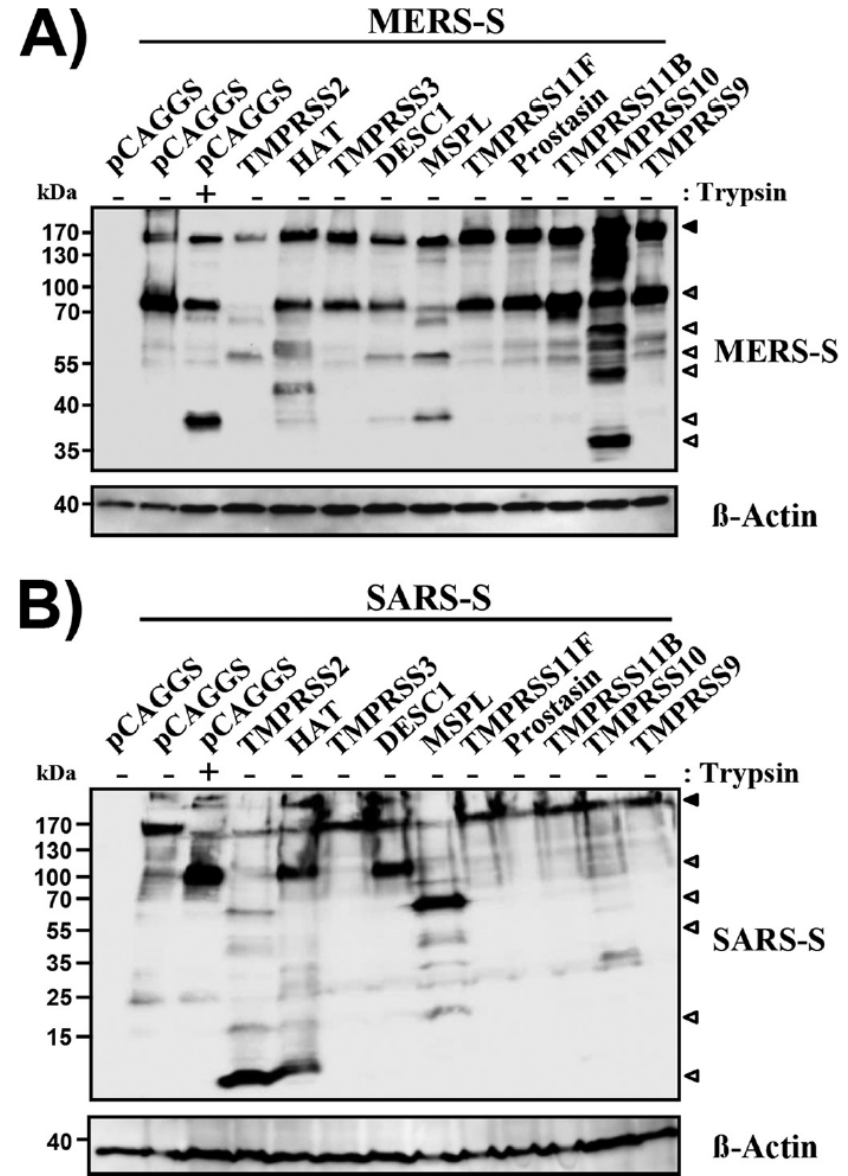

FIG 2 DESC1 and MSPL cleave the MERS- and SARS-CoV S proteins. 293T cells were cotransfected with plasmids encoding MERS- (A) or SARS- (B) CoV $\mathrm{S}$ with a C-terminal V5 tag and plasmids encoding the proteases indicated. Transfection of empty plasmid pCAGGS served as a negative control. At $48 \mathrm{~h}$ posttransfection, cells were either left untreated or treated with trypsin and lysates were analyzed by Western blotting with a V5-specific antibody. Expression of $\beta$-actin in cell lysates was determined as a loading control. The results are representative of three independent experiments with different plasmid preparations. Black-filled arrowheads, uncleaved S protein; gray-filled arrowheads, S2 subunit; white-filled arrowheads, C-terminal cleavage fragments.

coexpression of DESC1, MSPL, and TMPRSS10 (Fig. 2A), although differences in the size and number of cleavage fragments were observed. Similarly, cleavage of SARS-CoV S was observed in cells coexpressing DESC1 and MSPL (Fig. 2B). In contrast, TMPRSS10 did not facilitate the cleavage of SARS-CoV S. Thus, DESC1 and MSPL can process the MERS- and SARS-CoV S proteins while TMPRSS10 processes exclusively MERS-CoV S.

DESC1 and MSPL activate the $S$ proteins of the SARS- and MERS-CoVs. In order to examine whether S-protein cleavage results in activation, we assessed the impact of protease expression on S-protein-driven cell-cell fusion by using a previously reported cell-cell fusion assay (40). Expression of MERS-CoV S in effector cells allowed fusion with target cells transfected with a CD26encoding plasmid but not a control plasmid (Fig. 3A), as expected. Notably, the directed expression of CD26 on target cells was not required for robust MERS-CoV S-driven cell-cell fusion when the effector and target cell mixture was treated with trypsin (Fig. 3A), 


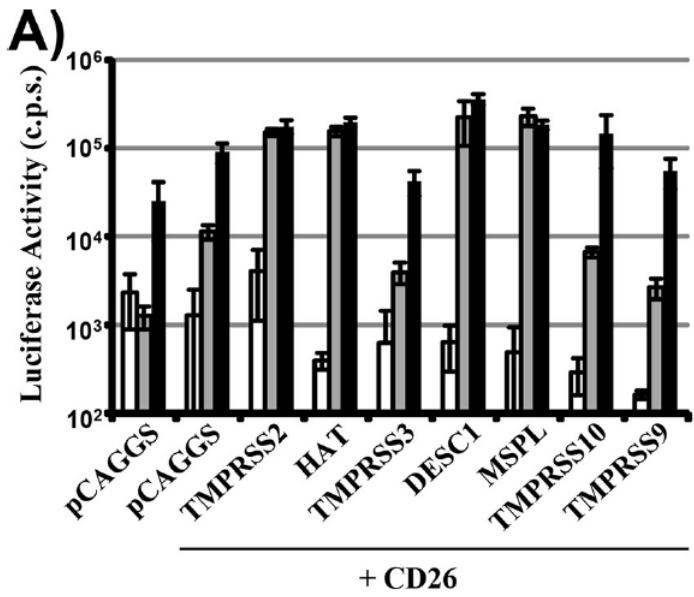

口pCAGGS 口MERS-S, PBS @ MERS-S, Trypsin

\section{B)}

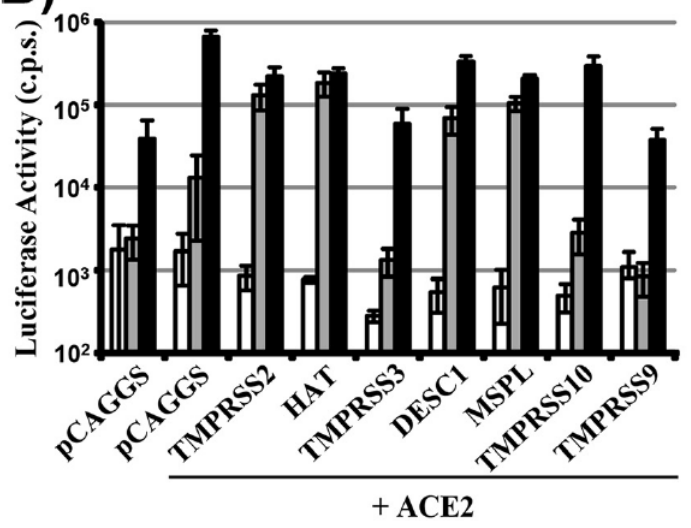

\section{口pCAGGS 口SARS-S, PBS $\square$ SARS-S, Trypsin}

FIG 3 DESC1 and MSPL activate the MERS- and SARS-CoV S proteins for cell-cell fusion. For the analysis of S protein-driven cell-cell fusion, effector $293 \mathrm{~T}$ cells were cotransfected with the pGAL4-VP16 expression plasmid and either the empty plasmid or a MERS- (A) or SARS- (B) CoV S expression plasmid. Subsequently, the effector cells were mixed with 293T target cells transfected with plasmids encoding the indicated receptors and proteases and a plasmid encoding luciferase under the control of a promoter with multiple GAL4 binding sites. The cell mixtures were then treated with either PBS or trypsin, and the luciferase activities in cell lysates were quantified at $48 \mathrm{~h}$ after cell mixing. The results of a representative experiment performed with triplicate samples are shown. Error bars indicate standard deviations. Similar results were observed in two independent experiments. c.p.s., counts per second.

in keeping with the finding that $293 \mathrm{~T}$ cells express small amounts of endogenous CD26 (46). These observations indicate that both receptor and protease expression levels limit MERS-CoV S-driven cell-cell fusion, a scenario similar to that previously reported for SARS-CoV S-mediated cell-cell fusion (47). Importantly, expression of TMPRSS2, HAT, DESC1, and MSPL activated MERS-CoV $\mathrm{S}$ with the same efficiency as trypsin (Fig. 3A), indicating that cleavage of MERS-CoV S by these proteases results in $\mathrm{S}$ protein activation. In contrast, TMPRSS10 expression failed to activate MERS-CoV S (Fig. 3A). Similar results were obtained upon the analysis of SARS-CoV S-driven cell-cell fusion, which was promoted by the expression of TMPRSS2, HAT, DESC1, and MSPL but not TMPRSS10 (Fig. 3B). Thus, DESC1 and MSPL can activate the MERS- and SARS-CoV S proteins and could contribute to syncytium formation in infected patients.

The cell-cell fusion assay allows the interaction of large cell surfaces on which large amounts of receptor and protease are expressed. Therefore, the assay is highly sensitive but might not fully mirror S-protein-driven virus-cell fusion. To assess whether the TTSPs studied can activate virion-associated S proteins, we analyzed whether TTSP expression rescues S-protein-driven viruscell fusion from blockade by an inhibitor of cathepsin B/L, proteases known to activate the MERS- and SARS-CoV S proteins for host cell entry (25-27). For this, we used lentiviral vectors pseudotyped with the SARS- and MERS-CoV S proteins, as previously described $(22,25)$. We found that incubation of 293T target cells with the cathepsin B/L inhibitor MDL 28170 reduced both MERS$\mathrm{CoV}$ S- (Fig. 4A) and SARS-CoV S-driven virus-cell fusion (Fig. 4B), as expected $(25,27)$. Expression of TMPRSS2 fully rescued MERS-CoV S- and SARS-CoV S-dependent virus-cell fusion from inhibition by MDL 28170, again in keeping with previously published results (22-25). In addition, MERS-CoV S-driven transduction was rescued by the expression of HAT, DESC1, and MSPL while similar effects were not measured for SARS-CoV Sdriven transduction. Collectively, these results indicate that DESC1 and MSPL can activate the MERS- and SARS-CoV S proteins for cell-cell fusion and could thus contribute to syncytium formation in patients. In addition, DESC1 and MSPL can activate MERS-CoV S for virus-cell fusion and may facilitate viral spreading in infected tissues. Why these proteases fail to activate SARS$\mathrm{CoV} \mathrm{S}$ for virus-cell fusion, at least in the system tested here, is unclear but most likely reflects a need for higher protease expression levels for SARS-CoV S activation than for MERS-CoV S activation.

DESC1 and MSPL cleave influenza virus $\mathrm{HA}$ of the $\mathrm{H} 1, \mathrm{H} 2$, and $\mathrm{H} 3$ subtypes and colocalize with $\mathrm{HA}$ in infected cells. In order to determine whether DESC1 and MSPL can activate FLUAV, we assessed their ability to cleave the HA proteins of viruses previously pandemic in humans. For this, the proteases were coexpressed with the HA proteins of A/South Carolina/1/ 1918 (H1N1), A/Singapore/1/57 (H2N2), and A/Hong Kong/1/ 1968 (H3N2) in transfected 293T cells. Cleavage of the HA precursor protein $\mathrm{HA} 0$ (as indicated by production of the $\mathrm{N}$-terminal cleavage product HA1) was observed upon the coexpression of TMPRSS2 and HAT (Fig. 5), in keeping with previously published data (15). In addition, the HAl processing product was observed upon the coexpression of DESC1 and MSPL (Fig. 5). In contrast, none of the other proteases tested facilitated HA cleavage.

In order to further determine whether DESC1 and MSPL can activate FLUAV, we next investigated whether these proteases colocalize with HA in infected cells. Immunofluorescence staining and visual inspection of COS-7 cells transfected to express TTSPs and infected with FLUAV revealed extensive colocalization of HA with TMPRSS2, DESC1, or MSPL (Fig. 6A). In contrast, TMPRSS 3 and HA barely colocalized, although the signals were close. This assessment was confirmed upon determination of the PCC for the HA and protease signals (PCC of 1, perfect positive correlation; PCC of -1 , perfect negative correlation). The HA signals correlated well with those of TMPRSS2, DESC1, and MSPL, indicating extensive colocalization, while little correlation was measured for the HA and TMPRSS3 signals (Fig. 6B). Thus, the cellular localizations of HA and DESC1 or MSPL overlap ex- 

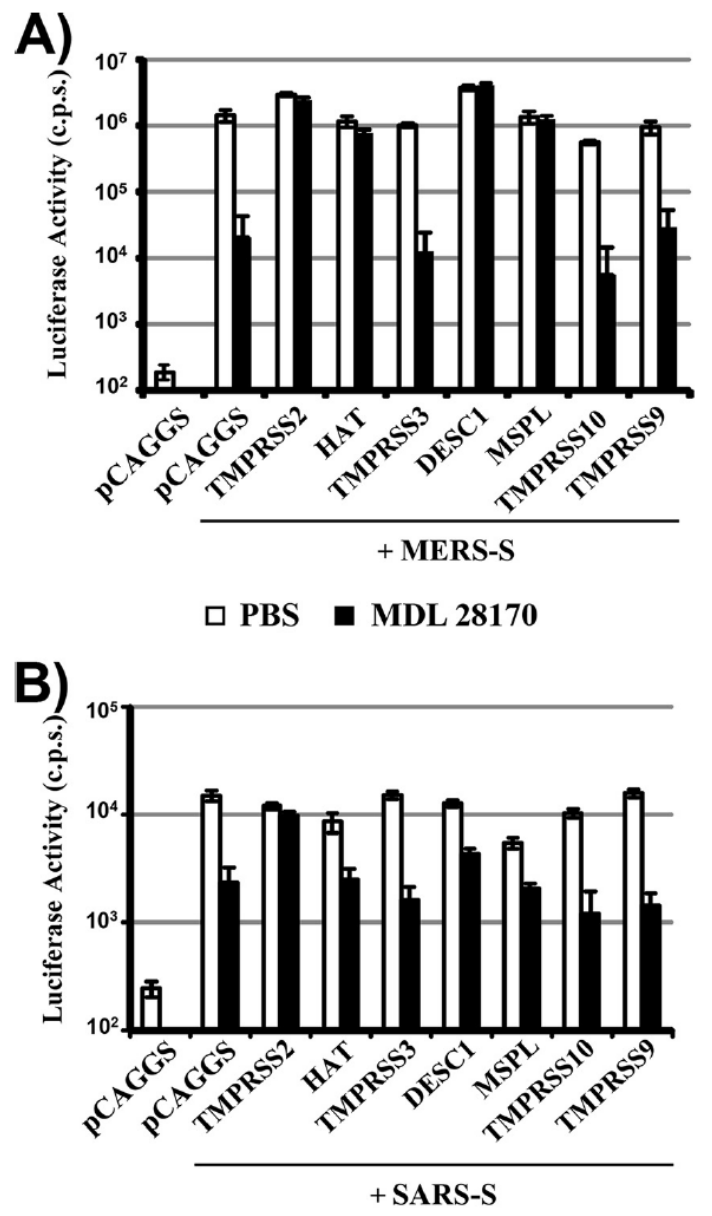

口 PBS M MDL 28170

FIG 4 DESC1 and MSPL activate MERS-CoV S for virus-cell fusion. For analysis of $\mathrm{S}$ protein-driven virus-cell fusion, the proteases indicated were coexpressed in 293T cells with CD26 (for MERS-CoV S-driven transduction, panel A) or ACE2 (for SARS-CoV S-driven transduction, panel B) and the cells were pretreated with medium containing DMSO or $10 \mu \mathrm{M}$ cathepsin B/L inhibitor MDL 28170. Subsequently, the cells were transduced with pseudotypes bearing MERS- (A) or SARS- (B) CoV S in the absence or presence of the inhibitor. The infection medium was replaced with fresh medium without inhibitor at $8 \mathrm{~h}$ posttransduction, and the luciferase activities in cell lysates were analyzed at $72 \mathrm{~h}$ posttransduction. The results of a representative experiment performed with triplicate samples are shown; error bars indicate standard deviations. Similar results were obtained in two to three independent experiments. c.p.s., counts per second.

tensively, suggesting that these proteases could cleave HA in infected cells.

DESC1 and MSPL activate the HA proteins of influenza viruses. Cleavage of a viral glycoprotein must not necessarily result in activation, as observed for MERS-CoV S cleavage by TMPRSS10 (Fig. 2 to 4). We therefore determined whether DESC1 and MSPL expression facilitates HA activation. First, we assessed if these proteases activate HA in the context of a lentiviral vector system. For this, a plasmid encoding the env-deficient lentiviral vector, FLUAV HA and NA, or the indicated proteases or an empty plasmid was transfected into 293T cells and the supernatants were analyzed for the transduction of target cells. All HA-

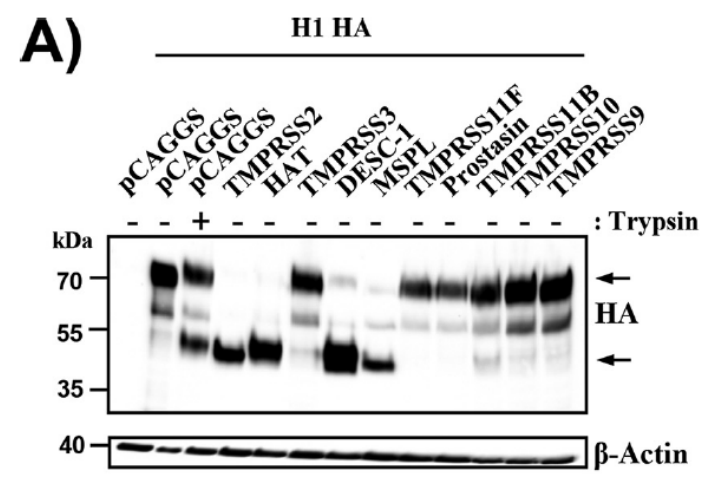

B) H2 HA
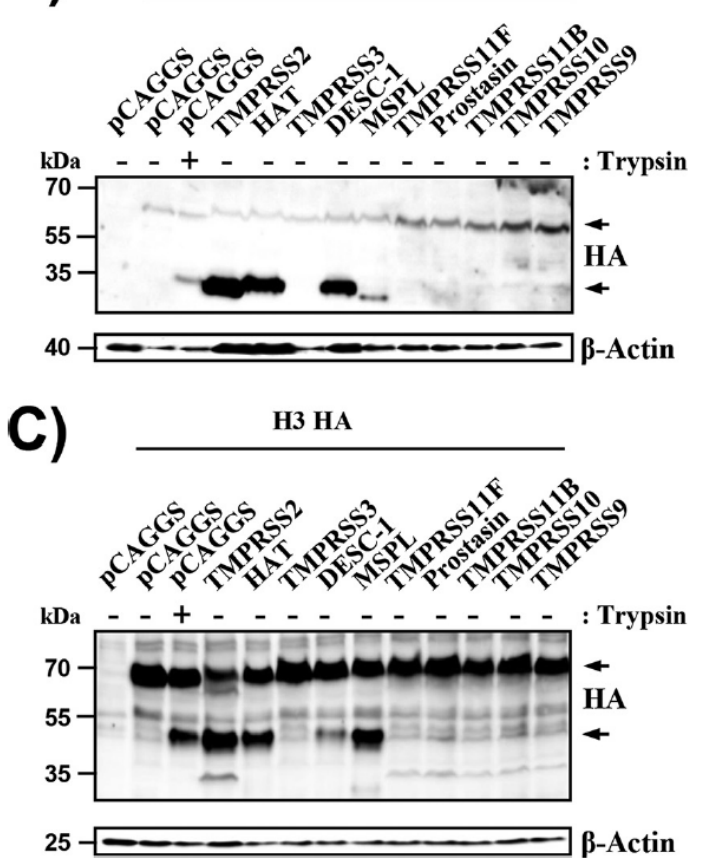

FIG 5 DESC1 and MSPL cleave the FLUAV HA. Expression plasmids encoding influenza virus HA subtypes $\mathrm{H} 1$ (A), H2 (B), and $\mathrm{H} 3$ (C) and the indicated proteases or empty plasmid pCAGGS were transiently cotransfected into 293T cells. The cells were then treated with PBS or trypsin, and HA cleavage was detected by Western blotting of cell lysates. Similar results were obtained in three independent experiments. The HA0 (upper arrow) and HA1 (lower arrow) subunits are indicated.

bearing vectors were able to efficiently and comparably transduce target cells upon treatment with trypsin, while trypsin treatment did not affect transduction mediated by VSV-G, as expected (33). Similarly, expression of TMPRSS2 and HAT rendered pseudotypes infectious, again in keeping with previously published data $(16,33)$, and the same effects were observed upon the expression of DESC1 and MSPL (Fig. 7A), indicating that these proteases can activate HA when expressed in virus-producing cells. In contrast, no activation of HA was observed when the proteases studied were expressed in viral target cells (data not shown), suggesting that DESC1 and MSPL fail to activate HA during viral entry. To address the activation of authentic FLUAV, we next examined the spreading of $\mathrm{A} / \mathrm{PR} / 8 / 34$ and A/Panama/2007/99 in 293T cells 


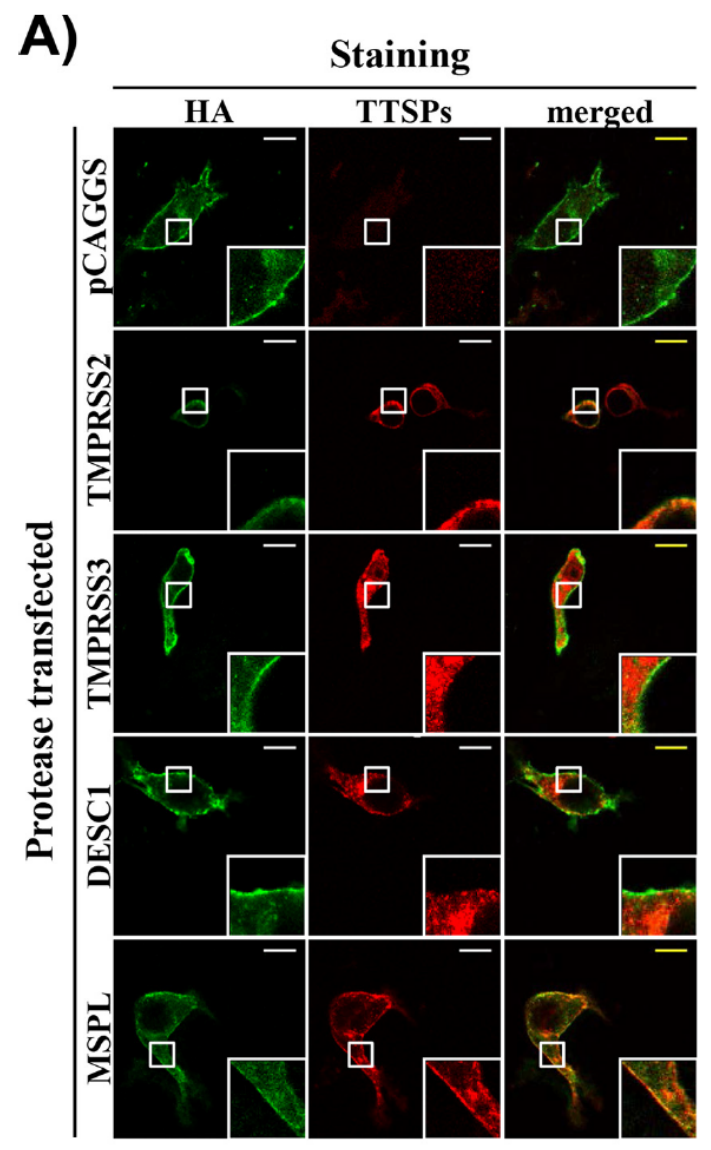

B)

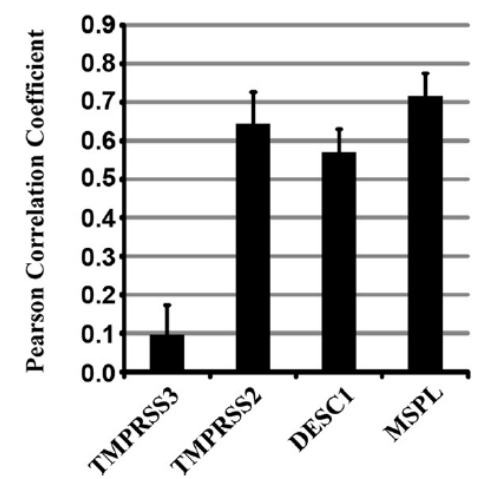

FIG 6 Analysis of protease and HA colocalization. (A) COS7 cells were transfected with plasmids encoding the indicated TTSPs or transfected with an empty plasmid (control). Subsequently, the cells were infected with FLUAV $\mathrm{A} / \mathrm{PR} / 8 / 34(\mathrm{H} 1 \mathrm{~N} 1)$ at an MOI of 1 . At $24 \mathrm{~h}$ postinfection, the cells were fixed with ice-cold methanol and influenza virus $\mathrm{HA}$ was detected by immunostain ing with rabbit-anti-HA and FITC-conjugated anti-rabbit antibodies (green). In parallel, proteases were detected with mouse anti-myc and Rhodamine Red-X-conjugated anti-mouse antibodies (red signal). White squares indicate examples of colocalization of HA and TTSPs (yellow signal) that were magnified $\times 4$. Similar results were obtained in three separate experiments. (B) The experiment was conducted as described for panel A, and colocalization of TTSPs and HA was determined by analyzing images with ImageJ software in combination with Just Another Colocalization Plugin, which allows calculation of the PCC, a measure of colocalization. The average PCC measured for three to five cells from separate experiments is shown, error bars indicate the standard errors of the means. transfected to express the proteases under study. In the absence of protease expression, viral spread was dependent on the presence of trypsin in the culture medium (Fig. 7B). If TMPRSS2, HAT, DESC1, and MSPL were produced in the target cells, efficient viral spread was measured (Fig. 7B), indicating that these proteases can activate HA in the context of infection with authentic FLUAV.

DESC1 and MSPL are expressed in the human lung. Activation of FLUAV, SARS-CoV, and MERS-CoV by DESC1 and MSPL in infected patients requires these proteases to be expressed in the human lung, the major viral target organ. In order to determine the expression of these proteases, we performed a quantitative RT-PCR analysis of two pools of RNA, each generated from normal lung tissue of four human male donors. TMPRSS2, MSPL, and DESC1 transcripts were detected in both pools although in different amounts. The expression of TMPRSS2 transcripts was most robust, but MSPL transcripts were also readily detectable. In contrast, expression of DESC1 mRNA was very low and thus might be confined to a limited subset of lung cells (Fig. 8). These results suggest that TMPRSS2, MSPL, and, to a lower degree, DESC1 are expressed in the lung and could thus facilitate the pulmonary spread of FLUAV and CoVs in human patients.

\section{DISCUSSION}

FLUAVs and emerging CoVs pose a significant threat to public health, and novel strategies for antiviral intervention are called for. TMPRSS2, a TTSP, is a potential target, since this enzyme activates FLUAV $(15,33)$ and CoVs $(22-24,26,28,29)$ in cell culture and was recently shown to be essential for FLUAV (H1N1, H7N9) spread and pathogenesis in mice (19-21). In contrast, the absence of TMPRSS2 had a much less profound effect in the context of infection with an $\mathrm{H} 3 \mathrm{~N} 2$ virus (19), suggesting that certain FLUAVs might employ TTSPs other than TMPRSS2 to ensure their activation in the host. Here, we show that DESC1 and MSPL are potential candidates, since these proteases are expressed in the human lung and activate the HA proteins of all of the FLUAV subtypes previously pandemic in humans. In addition, we demonstrate that DESC1 and MSPL activate the emerging MERS-CoV and might thus also be exploited by this highly pathogenic agent for spread in the infected host.

It was observed in the early 1970s that the FLUAV HA is cleaved by host cell proteases and that cleavage is essential for viral infectivity (48-50). Subsequently, a variety of secreted proteases that cleave and activate HA in cell culture was identified (12, 13, $51,52)$. These findings suggested that FLUAV might exploit multiple proteolytic systems present in the lung lumen to ensure its activation in the host. In contrast, recent studies indicate that FLUAV activation is cell associated (14) and mediated by TTSPs (15), with a single enzyme, TMPRSS2, being essential for the spread of the H1N1 and H7N9 FLUAV subtypes (19-21), while certain $\mathrm{H} 3 \mathrm{~N} 2$ viruses show little TMPRSS2 dependence. In fact, a recent study reported that an $\mathrm{HA}$ of the $\mathrm{H} 3$ subtype derived from avian FLUAV was fully resistant to cleavage by TMPRSS2 and HAT (53). The subtype or strain specificity of TMPRSS2 dependence is perhaps not unexpected, given the known differences in sequence and spatial presentation of the respective cleavage sites (54), and suggests that some viruses might use TTSPs other than TMPRSS2 to ensure their activation. In order to identify such enzymes, we focused our analysis on TTSPs known to be expressed in the murine and/or human lung, which comprise 


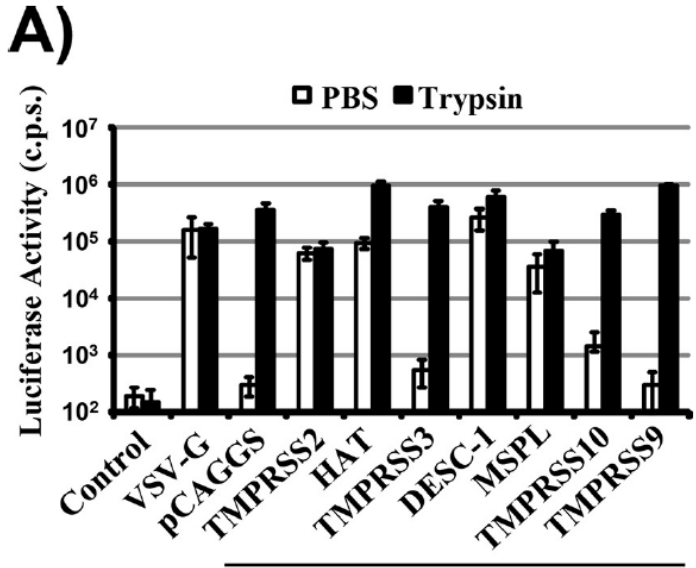

1918 HA/NA
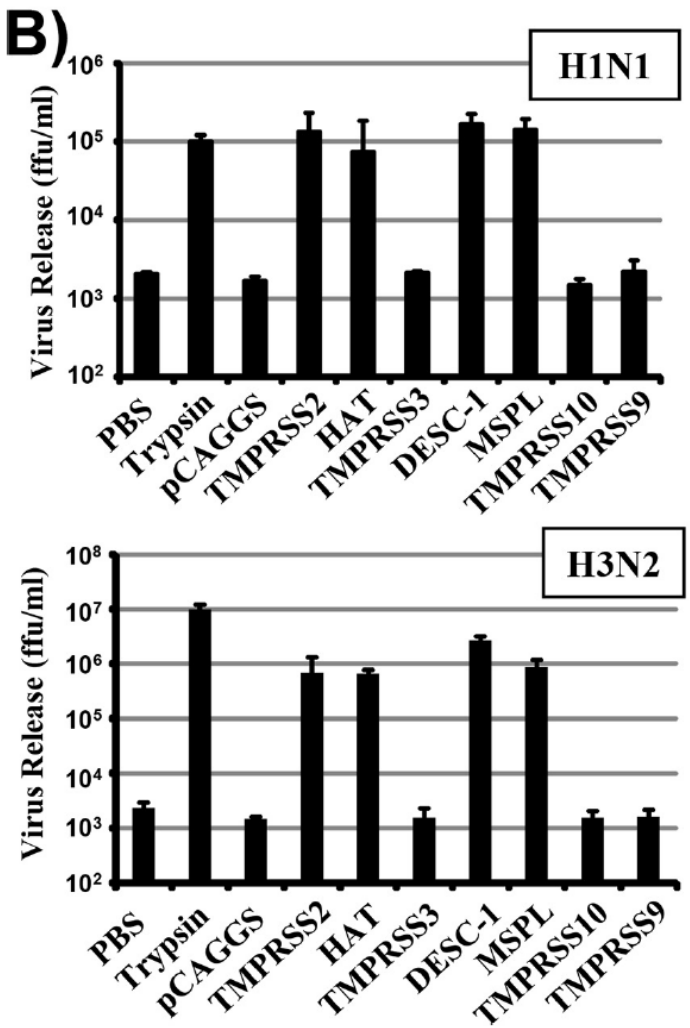

FIG 7 DESC1 and MSPL activate the FLUAV HA. (A) Lentiviral vectors bearing the HA and NA proteins of the H1N1 1918 influenza virus (1918 HA/NA) or VSV-G were generated in $293 \mathrm{~T}$ cells coexpressing the proteases indicated or transfected with empty plasmid pCAGGS. The culture supernatants were harvested treated with PBS or trypsin, and used for transduction of 293T target cells. Luciferase activities in the cell lysates were determined at $72 \mathrm{~h}$ posttransduction. The results of a representative experiment performed with triplicate samples are shown and were confirmed in two independent experiments. Error bars indicate standard deviations. (B) The proteases indicated were transiently expressed in 293T cells, and the cells were infected with FLUAV A/PR/8/34 (H1N1) at an MOI of 0.01 (top) or FLUAV A/Panama/2007/1999 (H3N2) at an MOI of 0.1 (bottom) and treated with either trypsin or PBS. At $48 \mathrm{~h}$ postinfection, viral spread was quantified as the release of infectious particles into the culture supernatants, as measured by a focus formation assay. The results of representative experiments performed with triplicate samples are shown. Error bars indicate standard deviations. Similar results were obtained in two separate experiments. c.p.s., counts per second; ffu, focus-forming units.

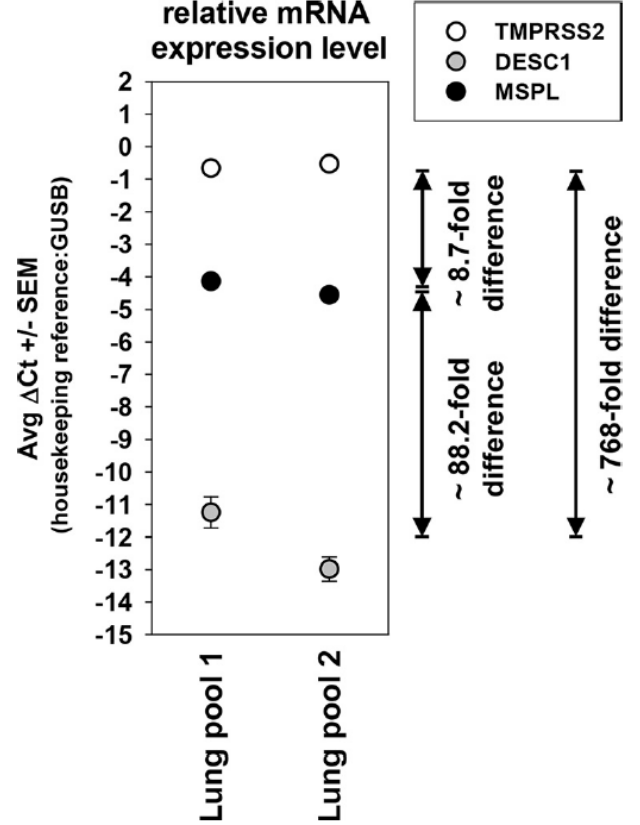

FIG 8 DESC1 and MSPL transcripts are expressed in the human lung. The presence of TMPRSS2, DESC1, and MSPL transcripts in two RNA pools each prepared from normal lung tissue of four human donors was assessed by quantitative PCR. Average $\Delta C_{T}$ values from triplicate measurements and the standard errors of the means are shown. The relative difference in the abundance of protease transcripts was estimated and is depicted as fold difference (an estimated 1.78-fold increase in amplified material per PCR cycle formed the basis for this calculation). Similar results were obtained in a separate experiment.

DESC1, MSPL, TMPRSS11F, prostasin, TMPRSS11B, TMPRSS9, and TMPRSS10 (31).

All of the TTSPs studied were expressed in transfected 293T cells, although substantial differences in expression efficiency were noted. Therefore, the possibility cannot be excluded that proteases found to be inactive in our study are able to activate viral glycoproteins when expressed at higher levels. TTSPs are synthesized as zymogens, and several can transit into an active form upon autocatalytic activation $(31,55)$. Activation of TTSPs results in the production of an $\mathrm{N}$-terminal cleavage fragment (linked to the C-terminal fragment via a disulfide bond), which was amenable to detection in our analysis because of the presence of an $\mathrm{N}$ terminal myc antigenic tag. This fragment was detected upon the expression of most of the proteases studied, indicating that the failure of certain proteases to activate viral glycoproteins was not due to a failure of these enzymes to transit into their catalytically active forms. The only exceptions were TMPRSS9, for which no evidence of activation was observed. Whether the absence of Nterminal cleavage products of TMPRSS9 in protease-expressing cells was due to instability of the respective fragments or to a genuine lack of protease activation remains to be determined.

TMPRSS2 was previously shown to activate the SARS- and MERS-CoV S proteins for cathepsin B/L-independent host cell entry (22-26), presumably by cleaving the $S$ proteins at or close to the cell surface. Cleavage of the MERS- and SARS-CoV S proteins by HAT has also been reported $(25,35)$. While the functional consequences of MERS-CoV S cleavage by HAT were unknown, 
SARS-CoV S cleavage by this protease was shown to activate the $\mathrm{S}$ protein for cell-cell fusion (35). The present study demonstrates that HAT can activate MERS-CoV S for entry into target cells in which cathepsin $\mathrm{B} / \mathrm{L}$ activity has been blocked. In addition, our work shows that MSPL and DESC1 activate both the MERS- and SARS-CoV S proteins for cell-cell fusion and MERS-CoV S for virus-cell fusion. Why MSPL, DESC1, and HAT failed to promote SARS-CoV S-driven virus-cell fusion is unclear. One explanation could be that activation of virion-associated SARS-CoV S requires higher protease expression levels than activation of MERS-CoV S. Alternatively, cleavage of SARS-CoV S at sites recognized by TMPRSS2, HAT, DESC1, and MSPL might be sufficient to activate the $\mathrm{S}$ protein for cell-cell fusion, while processing at one or more additional sites recognized only by TMPRSS2 might be required for virus-cell fusion. Which of the proteases able to activate the SARS- and MERS-CoV S proteins in cell culture contributes to viral spread in the infected host is unclear. A previous report demonstrated the coexpression of ACE2, the SARS-CoV receptor, and TMPRSS2/HAT in human respiratory epithelia (18), arguing for a role for these proteases in SARS-CoV infection. Whether DESC1 and MSPL are also present in viral target cells remains to be determined. Finally, the analysis of knockout mice, which have been reported for several TTSPs, including TMPRSS2 (56) and HAT (57), might help to define the relative contributions of the abovediscussed enzymes to viral spread.

The ability of several TTSPs to cleave and activate the SARSand MERS-CoV S proteins suggests a certain uniformity of the activation process. However, the analysis of the cleavage fragments indicates that this might not be the case. Thus, each protease produced different cleavage fragments, although the $\mathrm{S}$ protein fragments obtained upon MERS-CoV S and SARS-CoV S proteolysis by trypsin, HAT, and DESC1 exhibited a certain similarity. This is in keeping with the published observation that both trypsin and HAT cleave SARS-CoV S at R667 (35). Whether DESC1 requires the presence of the same residue for $\mathrm{S}$ protein cleavage remains to be determined, and also the sequences cleaved by TMPRSS 2 and MSPL are unknown. In this context, it should be emphasized that although different $\mathrm{S}$ protein-activating proteases produced different cleavage fragments, the robust, multiple cleavage of MERS-CoV S by TMPRSS10 did not result in S protein activation, underlining the existing but limited plasticity of the cleavage-activation process. Furthermore, it should be noted that $\mathrm{S}$ protein cleavage was analyzed in a cis format (i.e., protease and $\mathrm{S}$ protein were expressed in the same cell [Fig. 2]), which allows efficient detection of cleavage fragments, while $\mathrm{S}$ protein activation was determined in a trans format (protease and $\mathrm{S}$ protein were expressed in different cells [Fig. 3 and 4]). Our previous work demonstrated that cis and trans cleavage of SARS-CoV S by TMPRSS2 produces comparable, if not identical, $S$ protein fragments (22), indicating that the cleavage products observed in the present study should mirror those produced upon S protein activation. Finally, it has been suggested that binding of SARS-CoV S to its receptor might induce subtle conformational changes in the $S$ protein that increase its protease sensitivity $(27,58)$ and MERSCoV S binding to CD26 might have a similar effect. In the present study, S protein cleavage was assessed with cells expressing low, endogenous levels of receptor and we can thus not exclude the possibility that slightly different $S$ protein processing products would be generated in a cell system expressing high levels of receptor.
TMPRSS2 and HAT were initially identified as FLUAV-activating proteases (15) and were subsequently shown to activate CoVs $(22-24,29,35)$. Our results show that the novel S-proteinactivating enzymes DESC1 and MSPL also activate FLUAV, further strengthening the concept that different respiratory viruses might have adapted to use the same host cell enzymes to accomplish activation. Furthermore, the observation that MSPL and DESC1, like TMPRSS2 and HAT (15), can activate HA proteins of all of the FLUAV subtypes responsible for pandemics indicates a potential role in viral spread in vivo. Are MSPL and DESC1 the elusive proteases that allowed an $\mathrm{H} 3 \mathrm{~N} 2$ virus to spread in a TMPRSS2 knockout mouse? The observation that MSPL and DESC1 can activate $\mathrm{H} 1 \mathrm{~N} 1$ and $\mathrm{H} 3 \mathrm{~N} 2$ viruses seems to argue against this possibility. However, it is conceivable that only certain $\mathrm{H} 3 \mathrm{~N} 2$ viruses, but not $\mathrm{H} 1 \mathrm{~N} 1$ viruses, can target DESC1- and MSPL-positive cells in the lung. Another potential candidate for activation of $\mathrm{H} 3 \mathrm{~N} 2$ viruses is matriptase, a TTSP recently reported to activate FLUAV (59-61). However, cell culture studies demonstrated that this protease activates certain HAs of the $\mathrm{H} 1$ subtype while activation of $\mathrm{HAs}$ of the $\mathrm{H} 2$ and $\mathrm{H} 3$ subtypes was not observed $(60,61)$. Finally, it cannot be disregarded that although several TTSPs cleave HA, the cleavage rates might be different and might determine whether a protease can support viral spread in the host.

Here, we demonstrate that DESC1 and MSPL, the latter of which is known to activate certain FLUAVs with a multibasic cleavage site (62), activate the $\mathrm{S}$ proteins of emerging CoVs and human FLUAV. Whether DESC1 and MSPL contribute to viral spread in the infected host remains to be determined, and knockout mice, as well as specific protease inhibitors, might be useful tools for these endeavors. In this regard, it should be mentioned that camostat, an inhibitor of TMPRSS2, also blocks DESC1 and MSPL (data not shown) and can thus not be used for differential protease inhibition. Therefore, the generation of more specific inhibitors, as recently reported for TMPRSS2 (63), will be an important task. Finally, it is worth noting that FLUAV-activating TTSPs colocalized with HA, while an inactive TTSP did not, despite robust expression in the cellular system analyzed. It is therefore conceivable that the cellular localization of a TTSP, apart from its substrate specificity, might determine whether the protease can activate HA and other viral glycoproteins, a possibility that deserves further investigation.

\section{ACKNOWLEDGMENTS}

We thank Mikhail Matrosovich, Volker Czudai-Matwich, and Jan Baumann for $\mathrm{H} 2$ and $\mathrm{H} 3 \mathrm{HA}$ plasmids; Klaus Schughart and Thorsten Wolff for influenza viruses; Heike Hofmann-Winkler for critically reading the manuscript; and Oliver Dittrich-Breiholz for supervision of quantitative RT-PCR experiments.

This work was supported by the DFG (PO 716/6-1), the BMBF (01KI1005C), the Leibniz Graduate School for Emerging Infectious Diseases, and the Göttingen Graduate School for Neurosciences, Biophysics, and Molecular Biosciences (DFG grants GSC 226/1 and GSC 226/2).

\section{REFERENCES}

1. World Health Organization. 2014. Influenza (seasonal). World Health Organization, Geneva, Switzerland. http://www.who.int/mediacentre /factsheets/fs211/en/index.html.

2. Johnson NP, Mueller J. 2002. Updating the accounts: global mortality of the 1918-1920 "Spanish" influenza pandemic. Bull. Hist. Med. 76:105115. http://dx.doi.org/10.1353/bhm.2002.0022. 
3. World Health Organization. 2014. Summary of probable SARS cases with onset of illness from 1 November 2002 to 31 July 2003. World Health Organization, Geneva, Switzerland. http://www.who.int/csr/sars/country /table2004 04 21/en/index.html.

4. Zaki AM, van Boheemen S, Bestebroer TM, Osterhaus AD, Fouchier RA. 2012. Isolation of a novel coronavirus from a man with pneumonia in Saudi Arabia. N. Engl. J. Med. 367:1814-1820. http://dx.doi.org/10.1056 /NEJMoa1211721.

5. World Health Organization. 14 July 2014. Middle East respiratory syndrome coronavirus (MERS-CoV) - update. World Health Organization, Geneva, Switzerland. http://www.who.int/csr/don/2014_07_1 4_mers/en/.

6. Sun X, Whittaker GR. 2013. Entry of influenza virus. Adv. Exp. Med. Biol. 790:72-82. http://dx.doi.org/10.1007/978-1-4614-7651-1_4.

7. Belouzard S, Millet JK, Licitra BN, Whittaker GR. 2012. Mechanisms of coronavirus cell entry mediated by the viral spike protein. Viruses $4: 1011-$ 1033. http://dx.doi.org/10.3390/v4061011.

8. Heald-Sargent T, Gallagher T. 2012. Ready, set, fuse! The coronavirus spike protein and acquisition of fusion competence. Viruses 4:557-580. http://dx.doi.org/10.3390/v4040557.

9. Bertram S, Glowacka I, Steffen I, Kühl A, Pöhlmann S. 2010. Novel insights into proteolytic cleavage of influenza virus hemagglutinin. Rev. Med. Virol. 20:298-310. http://dx.doi.org/10.1002/rmv.657.

10. Simmons G, Zmora P, Gierer S, Heurich A, Pöhlmann S. 2013. Proteolytic activation of the SARS-coronavirus spike protein: cutting enzymes at the cutting edge of antiviral research. Antiviral Res. 100:605-614. http://dx.doi.org/10.1016/j.antiviral.2013.09.028.

11. Kido H, Okumura Y, Takahashi E, Pan HY, Wang S, Chida J, Le TQ, Yano M. 2008. Host envelope glycoprotein processing proteases are indispensable for entry into human cells by seasonal and highly pathogenic avian influenza viruses. J. Mol. Genet. Med. 3:167-175. http://dx.doi.org /10.4172/1747-0862.1000029.

12. Murakami M, Towatari T, Ohuchi M, Shiota M, Akao M, Okumura Y, Parry MA, Kido H. 2001. Mini-plasmin found in the epithelial cells of bronchioles triggers infection by broad-spectrum influenza A viruses and Sendai virus. Eur. J. Biochem. 268:2847-2855. http://dx.doi.org/10.1046/j .1432-1327.2001.02166.x.

13. Towatari T, Ide M, Ohba K, Chiba Y, Murakami M, Shiota M, Kawach M, Yamada H, Kido H. 2002. Identification of ectopic anionic trypsin I in rat lungs potentiating pneumotropic virus infectivity and increased enzyme level after virus infection. Eur. J. Biochem. 269:2613-2621. http://dx .doi.org/10.1046/j.1432-1033.2002.02937.x.

14. Zhirnov OP, Ikizler MR, Wright PF. 2002. Cleavage of influenza A virus hemagglutinin in human respiratory epithelium is cell associated and sensitive to exogenous antiproteases. J. Virol. 76:8682-8689. http://dx.do .org/10.1128/JVI.76.17.8682-8689.2002.

15. Böttcher E, Matrosovich T, Beyerle M, Klenk HD, Garten W, Matrosovich M. 2006. Proteolytic activation of influenza viruses by serine proteases TMPRSS2 and HAT from human airway epithelium. J. Virol. 80: 9896-9898. http://dx.doi.org/10.1128/JVI.01118-06.

16. Bertram S, Glowacka I, Blazejewska P, Soilleux E, Allen P, Danisch S, Steffen I, Choi SY, Park Y, Schneider H, Schughart K, Pöhlmann S. 2010. TMPRSS2 and TMPRSS4 facilitate trypsin-independent spread of influenza virus in Caco-2 cells. J. Virol. 84:10016-10025. http://dx.doi.org /10.1128/JVI.00239-10.

17. Böttcher-Friebertshäuser E, Stein DA, Klenk HD, Garten W. 2011 Inhibition of influenza virus infection in human airway cell cultures by an antisense peptide-conjugated morpholino oligomer targeting the hemagglutinin-activating protease TMPRSS2. J. Virol. 85:1554-1562. http://dx doi.org/10.1128/JVI.01294-10.

18. Bertram S, Heurich A, Lavender H, Gierer S, Danisch S, Perin P, Luca JM, Nelson PS, Pöhlmann S, Soilleux EJ. 2012. Influenza and SARScoronavirus activating proteases TMPRSS2 and HAT are expressed at multiple sites in human respiratory and gastrointestinal tracts. PLoS One 7(4):e35876. http://dx.doi.org/10.1371/journal.pone.0035876.

19. Hatesuer B, Bertram S, Mehnert N, Bahgat MM, Nelson PS, Pohlman S, Schughart K. 2013. Tmprss2 is essential for influenza H1N1 virus pathogenesis in mice. PLoS Pathog. 9(12):e1003774. http://dx.doi.org/10 .1371/journal.ppat.1003774.

20. Sakai K, Ami Y, Tahara M, Kubota T, Anraku M, Abe M, Nakajima N, Sekizuka T, Shirato K, Suzaki Y, Ainai A, Nakatsu Y, Kanou K, Nakamura K, Suzuki T, Komase K, Nobusawa E, Maenaka K, Kuroda M, Hasegawa H, Kawaoka Y, Tashiro M, Takeda M. 2014. The host protease
TMPRSS2 plays a major role in in vivo replication of emerging H7N9 and seasonal influenza viruses. J. Virol. 88:5608-5616. http://dx.doi.org/10 .1128/JVI.03677-13.

21. Tarnow C, Engels G, Arendt A, Schwalm F, Sediri H, Preuss A, Nelson PS, Garten W, Klenk HD, Gabriel G, Böttcher-Friebertshäuser E. 2014. TMPRSS2 is a host factor that is essential for pneumotropism and pathogenicity of H7N9 influenza A virus in mice. J. Virol. 88:4744-4751. http: //dx.doi.org/10.1128/JVI.03799-13.

22. Glowacka I, Bertram S, Muller MA, Allen P, Soilleux E, Pfefferle S, Steffen I, Tsegaye TS, He Y, Gnirss K, Niemeyer D, Schneider H, Drosten C, Pöhlmann S. 2011. Evidence that TMPRSS2 activates the severe acute respiratory syndrome coronavirus spike protein for membrane fusion and reduces viral control by the humoral immune response. J. Virol. 85:4122-4134. http://dx.doi.org/10.1128/JVI.02232-10.

23. Matsuyama S, Nagata N, Shirato K, Kawase M, Takeda M, Taguchi F. 2010. Efficient activation of the severe acute respiratory syndrome coronavirus spike protein by the transmembrane protease TMPRSS2. J. Virol. 84:12658-12664. http://dx.doi.org/10.1128/JVI.01542-10.

24. Shulla A, Heald-Sargent T, Subramanya G, Zhao J, Perlman S, Gallagher T. 2011. A transmembrane serine protease is linked to the severe acute respiratory syndrome coronavirus receptor and activates virus entry. J. Virol. 85:873-882. http://dx.doi.org/10.1128/JVI.02062-10.

25. Gierer S, Bertram S, Kaup F, Wrensch F, Heurich A, Kramer-Kuhl A, Welsch K, Winkler M, Meyer B, Drosten C, Dittmer U, HT von, Simmons G, Hofmann H, Pöhlmann S. 2013. The spike protein of the emerging betacoronavirus EMC uses a novel coronavirus receptor for entry, can be activated by TMPRSS2, and is targeted by neutralizing antibodies. J. Virol. 87:5502-5511. http://dx.doi.org/10.1128/JVI.00128-13.

26. Shirato K, Kawase M, Matsuyama S. 2013. Middle East respiratory syndrome coronavirus infection mediated by the transmembrane serine protease TMPRSS2. J. Virol. 87:12552-12561. http://dx.doi.org/10.1128 /JVI.01890-13.

27. Simmons G, Gosalia DN, Rennekamp AJ, Reeves JD, Diamond SL, Bates P. 2005. Inhibitors of cathepsin L prevent severe acute respiratory syndrome coronavirus entry. Proc. Natl. Acad. Sci. U. S. A. 102:1187611881. http://dx.doi.org/10.1073/pnas.0505577102.

28. Bertram S, Dijkman R, Habjan M, Heurich A, Gierer S, Glowacka I, Welsch K, Winkler M, Schneider H, Hofmann-Winkler H, Thiel V, Pöhlmann S. 2013. TMPRSS2 activates the human coronavirus 229E for cathepsin-independent host cell entry and is expressed in viral target cells in the respiratory epithelium. J. Virol. 87:6150-6160. http://dx.doi.org/10 $.1128 /$ JVI.03372-12.

29. Kawase M, Shirato K, van der Hoek L, Taguchi F, Matsuyama S. 2012. Simultaneous treatment of human bronchial epithelial cells with serine and cysteine protease inhibitors prevents severe acute respiratory syndrome coronavirus entry. J. Virol. 86:6537-6545. http://dx.doi.org/10 .1128/JVI.00094-12.

30. Shirogane Y, Takeda M, Iwasaki M, Ishiguro N, Takeuchi H, Nakatsu Y, Tahara M, Kikuta H, Yanagi Y. 2008. Efficient multiplication of human metapneumovirus in Vero cells expressing the transmembrane serine protease TMPRSS2. J. Virol. 82:8942-8946. http://dx.doi.org/10 1128/JVI.00676-08.

31. Antalis TM, Bugge TH, Wu Q. 2011. Membrane-anchored serine proteases in health and disease. Prog. Mol. Biol. Transl. Sci. 99:1-50. http://dx .doi.org/10.1016/B978-0-12-385504-6.00001-4.

32. Simmons G, Reeves JD, Rennekamp AJ, Amberg SM, Piefer AJ, Bates P. 2004. Characterization of severe acute respiratory syndrome-associated coronavirus (SARS-CoV) spike glycoprotein-mediated viral entry. Proc. Natl. Acad. Sci. U. S. A. 101:4240-4245. http://dx.doi.org/10.1073/pnas .0306446101 .

33. Chaipan C, Kobasa D, Bertram S, Glowacka I, Steffen I, Tsegaye TS, Takeda M, Bugge TH, Kim S, Park Y, Marzi A, Pöhlmann S. 2009. Proteolytic activation of the 1918 influenza virus hemagglutinin. J. Virol. 83:3200-3211. http://dx.doi.org/10.1128/JVI.02205-08.

34. Hofmann H, Geier M, Marzi A, Krumbiegel M, Peipp M, Fey GH, Gramberg T, Pöhlmann S. 2004. Susceptibility to SARS coronavirus S protein-driven infection correlates with expression of angiotensin converting enzyme 2 and infection can be blocked by soluble receptor. Biochem. Biophys. Res. Commun. 319:1216-1221. http://dx.doi.org/10 1016/j.bbrc.2004.05.114

35. Bertram S, Glowacka I, Muller MA, Lavender H, Gnirss K, Nehlmeier I, Niemeyer D, He Y, Simmons G, Drosten C, Soilleux EJ, Jahn O, Steffen I, Pöhlmann S. 2011. Cleavage and activation of the severe acute 
respiratory syndrome coronavirus spike protein by human airway trypsinlike protease. J. Virol. 85:13363-13372. http://dx.doi.org/10.1128/JVI .05300-11.

36. Cal S, Quesada V, Garabaya C, Lopez-Otin C. 2003. Polyserase-I, a human polyprotease with the ability to generate independent serine protease domains from a single translation product. Proc. Natl. Acad. Sci. U. S. A. 100:9185-9190. http://dx.doi.org/10.1073/pnas.1633392100.

37. Liao X, Wang W, Chen S, Wu Q. 2007. Role of glycosylation in corin zymogen activation. J. Biol. Chem. 282:27728-27735. http://dx.doi.org /10.1074/jbc.M703687200.

38. Heurich A, Hofmann-Winkler H, Gierer S, Liepold T, Jahn O, Pöhlmann S. 2014. TMPRSS2 and ADAM17 cleave ACE2 differentially and only proteolysis by TMPRSS2 augments entry driven by the severe acute respiratory syndrome coronavirus spike protein. J. Virol. 88:1293-1307. http://dx.doi.org/10.1128/JVI.02202-13.

39. Glaser L, Stevens J, Zamarin D, Wilson IA, Garcia-Sastre A, Tumpey TM, Basler CF, Taubenberger JK, Palese P. 2005. A single amino acid substitution in 1918 influenza virus hemagglutinin changes receptor binding specificity. J. Virol. 79:11533-11536. http://dx.doi.org/10.1128/JVI.79 $.17 .11533-11536.2005$

40. Hofmann H, Simmons G, Rennekamp AJ, Chaipan C, Gramberg T, Heck E, Geier M, Wegele A, Marzi A, Bates P, Pöhlmann S. 2006. Highly conserved regions within the spike proteins of human coronaviruses $229 \mathrm{E}$ and NL63 determine recognition of their respective cellular receptors. J. Virol. 80:8639-8652. http://dx.doi.org/10.1128/JVI.00560-06.

41. Simmons G, Reeves JD, Grogan CC, Vandenberghe LH, Baribaud F, Whitbeck JC, Burke E, Buchmeier MJ, Soilleux EJ, Riley JL, Doms RW, Bates P, Pöhlmann S. 2003. DC-SIGN and DC-SIGNR bind ebola glycoproteins and enhance infection of macrophages and endothelial cells. Virology 305:115-123. http://dx.doi.org/10.1006/viro.2002.1730.

42. Connor RI, Chen BK, Choe S, Landau NR. 1995. Vpr is required for efficient replication of human immunodeficiency virus type-1 in mononuclear phagocytes. Virology 206:935-944. http://dx.doi.org/10.1006 /viro.1995.1016

43. Matthaei M, Budt M, Wolff T. 2013. Highly pathogenic H5N1 influenza A virus strains provoke heterogeneous IFN-alpha/beta responses that distinctively affect viral propagation in human cells. PLoS One 8(2):e56659. http://dx.doi.org/10.1371/journal.pone.0056659.

44. Winkler M, Bertram S, Gnirß K, Nehlmeier I, Gawanbacht A, Kirchhoff F, Ehrhardt C, Ludwig S, Kiene M, Moldenhauer AS, Goedecke U, Karsten CB, Kuhl A, Pöhlmann S. 2012. Influenza A virus does not encode a tetherin antagonist with Vpu-like activity and induces IFNdependent tetherin expression in infected cells. PLoS One 7(8):e43337. http://dx.doi.org/10.1371/journal.pone.0043337.

45. Bolte S, Cordelieres FP. 2006. A guided tour into subcellular colocalization analysis in light microscopy. J. Microsc. 224:213-232. http://dx.doi .org/10.1111/j.1365-2818.2006.01706.x.

46. Wilson CH, Abbott CA. 2012. Expression profiling of dipeptidyl peptidase 8 and 9 in breast and ovarian carcinoma cell lines. Int. J. Oncol. 41:919-932. http://dx.doi.org/10.3892/ijo.2012.1522.

47. Simmons G, Bertram S, Glowacka I, Steffen I, Chaipan C, Agudelo J, Lu K, Rennekamp AJ, Hofmann H, Bates P, Pöhlmann S. 2011. Different host cell proteases activate the SARS-coronavirus spike-protein for cellcell and virus-cell fusion. Virology 413:265-274. http://dx.doi.org/10 .1016/j.virol.2011.02.020

48. Klenk HD, Rott R. 1973. Formation of influenza virus proteins. J. Virol. $11: 823-831$

49. Klenk HD, Rott R, Orlich M, Blodorn J. 1975. Activation of influenza A viruses by trypsin treatment. Virology 68:426-439. http://dx.doi.org/10 .1016/0042-6822(75)90284-6.
50. Lazarowitz SG, Compans RW, Choppin PW. 1973. Proteolytic cleavage of the hemagglutinin polypeptide of influenza virus. Function of the uncleaved polypeptide HA. Virology 52:199-212.

51. Kido H, Yokogoshi Y, Sakai K, Tashiro M, Kishino Y, Fukutomi A, Katunuma N. 1992. Isolation and characterization of a novel trypsin-like protease found in rat bronchiolar epithelial Clara cells. A possible activator of the viral fusion glycoprotein. J. Biol. Chem. 267:13573-13579.

52. Kido H, Okumura Y, Yamada H, Le TQ, Yano M. 2007. Proteases essential for human influenza virus entry into cells and their inhibitors as potential therapeutic agents. Curr. Pharm. Des. 13:405-414. http://dx.do .org/10.2174/138161207780162971.

53. Galloway SE, Reed ML, Russell CJ, Steinhauer DA. 2013. Influenza HA subtypes demonstrate divergent phenotypes for cleavage activation and $\mathrm{pH}$ of fusion: implications for host range and adaptation. PLoS Pathog. 9(2):e1003151. http://dx.doi.org/10.1371/journal.ppat.1003151.

54. Lu X, Shi Y, Gao F, Xiao H, Wang M, Qi J, Gao GF. 2012. Insights into avian influenza virus pathogenicity: the hemagglutinin precursor $\mathrm{HA} 0$ of subtype $\mathrm{H} 16$ has an alpha-helix structure in its cleavage site with inefficient HA1/HA2 cleavage. J. Virol. 86:12861-12870. http://dx.doi.org/10 1128/JVI.01606-12.

55. Bugge TH, Antalis TM, Wu Q. 2009. Type II transmembrane serine proteases. J. Biol. Chem. 284:23177-23181. http://dx.doi.org/10.1074/jbc .R109.021006.

56. Kim TS, Heinlein C, Hackman RC, Nelson PS. 2006. Phenotypic analysis of mice lacking the Tmprss2-encoded protease. Mol. Cell. Biol. 26:965975. http://dx.doi.org/10.1128/MCB.26.3.965-975.2006.

57. Sales KU, Hobson JP, Wagenaar-Miller R, Szabo R, Rasmussen AL, Bey A, Shah MF, Molinolo AA, Bugge TH. 2011. Expression and genetic loss of function analysis of the HAT/DESC cluster proteases TMPRSS11A and HAT. PLoS One 6(8):e23261. http://dx.doi.org/10.1371/journal.pone .0023261 .

58. Matsuyama S, Ujike M, Morikawa S, Tashiro M, Taguchi F. 2005. Protease-mediated enhancement of severe acute respiratory syndrome coronavirus infection. Proc. Natl. Acad. Sci. U. S. A. 102:12543-12547. http://dx.doi.org/10.1073/pnas.0503203102.

59. Baron J, Tarnow C, Mayoli-Nussle D, Schilling E, Meyer D, Hammami M, Schwalm F, Steinmetzer T, Guan Y, Garten W, Klenk HD, BöttcherFriebertshäuser E. 2013. Matriptase, HAT, and TMPRSS2 activate the hemagglutinin of H9N2 influenza A viruses. J. Virol. 87:1811-1820. http: //dx.doi.org/10.1128/JVI.02320-12.

60. Beaulieu A, Gravel E, Cloutier A, Marois I, Colombo E, Desilets A Verreault C, Leduc R, Marsault E, Richter MV. 2013. Matriptase proteolytically activates influenza virus and promotes multicycle replication in the human airway epithelium. J. Virol. 87:4237-4251. http://dx.doi.org /10.1128/JVI.03005-12.

61. Hamilton BS, Gludish DW, Whittaker GR. 2012. Cleavage activation of the human-adapted influenza virus subtypes by matriptase reveals both subtype and strain specificities. J. Virol. 86:10579-10586. http://dx.doi .org/10.1128/JVI.00306-12.

62. Okumura Y, Takahashi E, Yano M, Ohuchi M, Daidoji T, Nakaya T, Böttcher E, Garten W, Klenk HD, Kido H. 2010. Novel type II transmembrane serine proteases, MSPL and TMPRSS13, proteolytically activate membrane fusion activity of the hemagglutinin of highly pathogenic avian influenza viruses and induce their multicycle replication. J. Virol. 84:5089-5096. http://dx.doi.org/10.1128/JVI.02605-09.

63. Mever D, Sielaff F, Hammami M, Böttcher-Friebertshäuser E, Garten W, Steinmetzer T. 2013. Identification of the first synthetic inhibitors of the type II transmembrane serine protease TMPRSS2 suitable for inhibition of influenza virus activation. Biochem. J. 452:331-343. http://dx.doi .org/10.1042/BJ20130101. 


\title{
Second manuscript
}

The stem domain of type II transmembrane serine proteases determines the activation of viral glycoproteins and is a potential target for intervention

\author{
Zmora. P., Moldenhauer, A.S., Pöhlmann, S.
}

Journal of Virology, submitted on 12.10.2015 (submission number JVI02619-15)

\section{Individual contribution:}

Design and conduct of the experiments. Construction of TMPRSS2/TMPRSS3 chimeras (Fig. 1A). Analysis of TTSPs chimeras expression via Western blotting (Fig. 1B). Determination whether constructed chimeras cleave HA (Fig. 2A) and whether cleavage results in the virus activation (Fig. 2B). Analysis of coronavirus S protein cleavage (Fig. 3A) and activation for virus-cell fusion (Fig. 3B). Determination of the role of stem region in the HA cleavage and activation (Fig. 4). Analysis of TMPRSS4/TMPRSS3 chimeras expression and activity (Fig. 5). The microscopic analysis of HA and TTSPs chimeras cellular localization (Fig. 6). Identification of stem region inhibitor (Fig. 7). Writing parts of the manuscript (Material and Methods, Results) 


\section{The stem domain of type II transmembrane serine proteases determines the activation of viral glycoproteins and is a potential target for antiviral intervention}

Pawel Zmora, Anna-Sophie Moldenhauer, Stefan Pöhlmann

Infection Biology Unit, German Primate Center, Göttingen, Germany.

Address correspondence to Stefan Pöhlmann, spoehlmann@dpz.eu

Running title: Influenza virus activation by TTSPs 


\section{ABSTRACT}

The type II transmembrane serine protease (TTSP) TMPRSS2 activates the influenza A virus hemagglutinin (FLUAV HA) and coronavirus spike proteins (CoV S) by proteolytic cleavage and is essential for FLUAV spread and pathogenesis in mice. In addition, several related TTSPs activate HA in cell culture while other enzymes of the same family are inactive, despite an identical domain organization and up to $40 \%$ sequence homology. Here, we asked which domains in TTSPs determine cleavage and activation of viral glycoproteins. Analysis of chimeric proteases revealed that the stem domain determines whether TTSPs can cleave and activate HA and CoV S proteins. Moreover, confocal microscopy provided evidence that the stem domain controls HA activation by determining protease colocalization with HA. Finally, we found that HA activation by TTSPs containing a scavenger receptor A (SR-A) domain in their stem region can be inhibited by dextran sulphate, a known SR-A ligand. These results identify the stem domain of TTSPs as a key determinant of proteolytic processing of viral glycoproteins and possibly other ligands. Moreover, our findings provide evidence that the SRA domain within the stem is a target for intervention, which has implications for the development of novel antiviral strategies. 


\section{IMPORTANCE}

The efficacy of antivirals for treatment of influenza is compromised by viral resistance. The inhibition of cellular factors essential for influenza virus replication might suppress viral spread without inducing resistance development. The cellular protease TMPRSS2 is a potential target: TMPRSS2 activates the influenza virus hemagglutinin (HA) and is essential for viral pathogenesis in mice. The successful exploitation of TMPRSS2 for antiviral therapy requires a thorough understanding of how TMPRSS2 recognizes HA. Here, we show that the stem region of TMPRSS2 is the key determinant of $\mathrm{HA}$ activation, because it controls the ability of TMPRSS2 to colocalize with HA. Moreover, we provide evidence that HA activation can be blocked by inhibitors targeting a scavenger receptor domain located in the stem region. These findings identify the stem region as a major determinant of ligand recognition by TMPRSS2 and might facilitate efforts to devise agents which block TMPRSS2 with high specificity and efficiency. 


\section{INTRODUCTION}

The ability of influenza A viruses (FLUAV) to constantly adapt to immune pressure results in annually recurring influenza epidemics, which pose a significant health threat, in particular to infants and the elderly: 250000 to 500000 annual deaths are attributed to influenza epidemics and are associated with costs of 87.1 billion USD per year in the US alone $(1,2)$. Moreover, the introduction of new FLUAV into the human population or the reassortment of human and animal FLUAV may result in influenza pandemics with potentially devastating consequences, as exemplified by the pandemic in 1918, which is believed to have claimed 30 to 50 million lives (3). Preventive and therapeutic options against influenza are in place $(4,5)$ but have significant shortcomings: Vaccination protects against epidemic but not pandemic influenza and currently available therapeutics, which target the viral proteins neuraminidase and $\mathrm{M} 2$, are plagued by frequent emergence of resistant viruses (6). Therefore, new therapeutic options against FLUAV have to be devised which display a high barrier against resistance development and host cell factors which are essential for viral spread but dispensable for cellular survival are attractive targets for such endeavours.

The FLUAV hemagglutinin (HA) protein is inserted into the viral envelope and facilitates infectious viral entry into target cells (7). HA is generated as an inactive precursor protein in the constitutive secretory pathway of infected cells and is only converted into its active form upon processing by host cell proteases $(8,9)$. Consequently, the activity of the responsible proteases is essential for viral infectivity. It has long been believed that a multitude of proteases secreted into the lung lumen facilitate HA activation in the host $(10,11)$. However, recent studies demonstrate that the type II transmembrane serine protease TMPRSS2 activates FLUAV upon directed and endogenous expression (12-14), that TMPRSS2 is expressed in susceptible cells in the human aerodigestive tract (15) and that TMPRSS2 is essential for FLUAV spread and pathogenesis in mice (16-18). Moreover, polymorphisms in TMPRSS2 which increase protease expression were found to be associated with susceptibility to severe influenza (19). Finally, several studies indicate that viruses other than FLUAV, including SARS- and MERS-coronavirus (SARS-CoV, MERS-COV) (20-24) and hepatitis 
C virus (25) can be activated by TMPRSS2. Collectively, TMPRSS2 is a host factor essential for spread of FLUAV and potentially other viruses but is dispensable for normal development and homeostasis (26) and thus constitutes an attractive target for therapeutic intervention.

Cell culture studies revealed that several TTSPs other than TMPRSS2 can activate HA, including HAT, TMPRSS4, DESC1 and MSPL $(14,27,28)$, and it is at present unclear whether these enzymes can contribute to viral spread in the host under certain conditions. Moreover, more than 8 TTSPs were identified which failed to activate HA despite robust expression in cell culture $(12,28)$ and despite exhibiting the same domain organization as TMPRSS2 as well as considerable sequence homology. For instance, TMPRSS3, like TMPRSS2, contains a cytoplasmic, transmembrane, stem and protease domain and the protease domains of these two enzymes share $71 \%$ amino acid homology. Still, TMPRSS3 fails to activate HA in cells engineered to express high levels of the protease $(12,28)$. Therefore, we investigated which domains in TTSPs determine the ability to activate HA and we asked if this domain/these domains can be targeted for antiviral intervention.

Our results show that the stem region in TTSPs determines HA activation by controlling the ability of these enzymes to colocalize with HA. Moreover, we demonstrate that HA activation by TTSPs which encode a scavenger receptor A (SR-A) domain in their stem can be inhibited by dextran sulphate, a known ligand of SR-A $(29,30)$. Our results define the stem region as a central determinant of FLUAV activation and ligand specificity of TTSPs and might have implications for the development of novel therapeutic options. 


\section{MATERIALS AND METHODS}

Cell culture. The cell lines 293T, Calu-3, Caco-2 and COS-7 were grown in Dulbecco's modified Eagle's medium (DMEM, PAN Biotech) while MDCK cells were grown in minimum essential medium (MEM, Gibco). All media were supplemented with $10 \%$ fetal bovine serum (FBS, Biochrome) and $100 \mathrm{U} / \mathrm{ml}$ penicillin and streptomycin (PAN Biotech).

Plasmids. The plasmids encoding TMPRSS2, TMPRSS3, TMPRSS4, HA of subtype H1 and H3, the spike (S) proteins of SARS-CoV (SARS-S) and MERS-CoV (MERS-S), ACE2 and DPP4 were described previously $(12,20,21,28)$. Chimeric proteases with and without $\mathrm{N}$-terminal myc antigenic tag were constructed using overlap extension PCR. PCR amplicons were cloned into pCAGGS (31) via EcoRI, XhoI (TMPRSS2/TMPRSS3 chimeras) and KpnI, XhoI (TMPRSS4/TMPRSS3 chimeras) and the sequences were verified by automated sequencing. Detailed cloning strategies as well as primer sequences are available upon request.

TTSP expression and autocatalytic activation. For analysis of expression and autocatalytic activation of TTSP chimeras, the plasmids encoding the proteases equipped with an $\mathrm{N}$-terminal myc tag or empty plasmid as a control were transiently transfected into $293 \mathrm{~T}$ cells, previously seeded into 6-well plates at a density of $2.8 \times 10^{5}$ cells/well. After overnight incubation, the medium was replaced with fresh DMEM and at $48 \mathrm{~h}$ post transfection the cells were washed with phosphate-buffered saline (PBS), resuspended in $100 \mu \mathrm{l}$ of $2 \mathrm{x}$ sodium dodecyl sulphate (SDS) loading buffer per well and then heated at $95^{\circ} \mathrm{C}$ for $30 \mathrm{~min}$. Protein samples were separated by SDS-PAGE, and blotted onto a nitrocellulose membrane (Hartenstein). Protease expression was detected using a mouse anti-myc antibody (Biomol) as the primary antibody and a horseradish peroxidase (HRP)-coupled antibody (Dianova) as the secondary antibody. Expression of $\beta$-actin, detected with anti- $\beta$-actin antibody 
(Sigma Aldrich), served as a loading control. A commercially available ECL Prime Western blotting detection kit (Amersham) was used to detect the bound antibodies and the image acquisition was performed with a ChemoCam Imager (Intas).

Cleavage of viral glycoproteins by TTSPs. To determine cleavage of FLUAV HA by wt and chimeric TTSPs, 293T cells were seeded in 6-well plates at a concentration of $2.8 \times 10^{5}$ cells/well and cultured for $24 \mathrm{~h}$. Subsequently, the cells were cotransfected with $6 \mu \mathrm{g}$ of plasmid encoding FLUAV $\mathrm{HA}$ of the $\mathrm{H} 1$ or $\mathrm{H} 3$ subtype and $0.3 \mu \mathrm{g}$ of plasmid encoding the indicated proteases, employing the calcium phosphate transfection protocol. At $16 \mathrm{~h}$ post transfection, the medium was changed and at 48 $\mathrm{h}$ post transfection the cells were harvested in PBS, treated with PBS or $250 \mu \mathrm{g} / \mathrm{ml}$ tosylsulfonyl phenylalanyl chloromethyl ketone (TPCK) treated trypsin (Sigma Aldrich) for 10 min at $37^{\circ} \mathrm{C}$ and processed for Western blot analysis as described above. FLUAV HA of the H1 subtype was detected with a goat anti-FLUAV polyclonal antibody (Milipore) while HA of the H3 subtype was detected with rabbit anti-H3 HA serum (Immune Technology).

For analysis of SARS-S and MERS-S cleavage by TTSPs, 293T cells were seeded in 6-well plates at a concentration of $2.88 \times 10^{5}$ cells/well, cultured for $24 \mathrm{~h}$ and then calcium-phosphate transfected with $6 \mu \mathrm{g}$ of plasmid encoding SARS-S and MERS-S, both equipped with a C terminal V5 tag. Simultaneously, 293T cells were prepared in the same way and were transfected with empty plasmid (pCAGGS) or plasmids encoding ACE2 or DPP4, respectively, and the proteases. At $16 \mathrm{~h}$ post transfection the medium was changed and at $24 \mathrm{~h}$ post transfection $\mathrm{S}$ protein and receptor/protease expressing cells mixed, cultivated for 24 , treated with PBS or TPCK trypsin and processed for Western blot analysis as described above. SARS-S and MERS-S were detected by staining with mouse monoclonal antibody reactive against the V5 tag (Invitrogen), followed by incubation with an HRP-coupled anti-mouse secondary antibody (Dianova).

To analyze cleavage inhibition by dextran sulphate, an SR-A inhibitor $(29,30), 293 \mathrm{~T}$ cells were transfected with plasmids encoding $\mathrm{HA}$ and proteases, as described above. At $8 \mathrm{~h}$ post 
transfection, the medium was replaced with fresh DMEM supplemented with PBS, $100 \mu \mathrm{g} / \mathrm{ml}$ dextran sulphate (Sigma Aldrich) or $100 \mu \mathrm{g} / \mathrm{ml}$ chondroitin sulphate (Sigma Aldrich). At $48 \mathrm{~h}$ post transfection the cells were harvested and processed for Western blot analysis as described above. The quantification of HA cleavage was performed with Fiji software (32).

FLUAV production and infection experiments. A/PR/8/34 (H1N1) was obtained from collaborators and was previously described (16). A/Panama/2007/99 (H3N2) was reconstituted from a previously described 8-plasmid system (33). Both viruses were propagated in the chorio-allantoic cavity of 10day-old embryonated hen eggs (Valo Biomedia Gmbh, Germany) for $48 \mathrm{~h}$ at $37^{\circ} \mathrm{C}$. Thereafter, the eggs were chilled overnight at $4{ }^{\circ} \mathrm{C}$ and the allantoic fluid was harvested. For infection experiments, $293 \mathrm{~T}$ cells were seeded in 12 -well plates at a density of $1.4 \times 10^{5}$ cells/well. After $24 \mathrm{~h}$, the cells were transfected with $3 \mu \mathrm{g}$ expression plasmids encoding the indicated proteases or empty plasmid using the calcium phosphate transfection method. The transfection medium was replaced by fresh medium after an overnight incubation. At $24 \mathrm{~h}$ post transfection, the culture medium was removed and the cells incubated with infection medium (DMEM supplemented with $0,2 \%$ BSA) containing A/PR/8/34 (H1N1) at an MOI of 0.01 or A/Panama/2007/99 (H3N2) at an MOI of 0.1. After $1 \mathrm{~h}$ of incubation at $37^{\circ} \mathrm{C}$, the infection medium was removed, the cells were gently washed with PBS and fresh infection medium was added. Culture supernatants were collected at $48 \mathrm{~h}$ post infection and the infectious units were quantified by focus formation assay, as described below. To assess inhibition of TTSPs by dextran sulphate and chondroitin sulphate, as control, the 293T cells were transfected with indicated proteases and infected with $\mathrm{A} / \mathrm{PR} / 8 / 34(\mathrm{H} 1 \mathrm{~N} 1)$ at an MOI of 0.01 , as described above. After infection, the medium was replaced with fresh infection medium supplemented with PBS, $100 \mu \mathrm{g} / \mathrm{ml}$ dextran sulphate sodium salt (Sigma Aldrich) or $100 \mu \mathrm{g} / \mathrm{ml}$ chondroitin sulfate A sodium salt from bovine trachea (Sigma Aldrich) and infectivity in culture supernatants was determined at $48 \mathrm{~h}$ post infection. To assess inhibition of FLUAV in Caco-2 or Calu-3 cells, which express endogenous TMPRSS2 (12, 13), the cells were seeded in 12 -well plates at a density of $1.4 \times 10^{5}$ cells/well. After $24 \mathrm{~h}$, the cells were infected with A/PR/8/34 (H1N1) at an MOI of 0.01, as described above. After 1h of incubation 
with virus, the cells were washed with PBS and fresh infection medium supplemented with increasing amount of dextran sulphate or chondroitin sulphate was added. Culture supernatants were collected at $48 \mathrm{~h}$ post infection.

Focus formation assay. The infectious units within culture supernatants were quantified by focus formation assay, as described previously (34). In brief, serial 5-fold dilution of samples were prepared in infection medium (DMEM with 1\% P/S and 0.1\% BSA) and added to MDCK cells. After $1 \mathrm{~h}$ of incubation, the medium was replaced by infection medium containing Avicel overlay and $2.5 \mu \mathrm{g} / \mathrm{ml}$ $\mathrm{N}$-acetylated trypsin (Sigma), and the cells were incubated for $24 \mathrm{~h}$. Subsequently, the cells were fixed with 4\% formalin in PBS, and incubated for $1 \mathrm{~h}$ with anti-FLUAV (Milipore), followed by $1 \mathrm{~h}$ incubation with anti-goat-HRP antibodies (Dianova), and $10 \mathrm{~min}$ incubation with True Blue substrate (KPL). Foci were counted and viral titers were calculated as focus forming units (FFU) per ml of culture supernatant.

Colocalization of HA and TTSPs. The colocalization of TTSPs and HA was determined as described previously (28). In brief, COS-7 cells were transiently transfected with $6 \mu \mathrm{g}$ of plasmids encoding the indicated proteases equipped with $\mathrm{N}$-terminal myc tag or empty plasmid as control. After overnight incubation, the transfection medium was replaced with fresh DMEM, and at $24 \mathrm{~h}$ post transfection, the cells were washed with PBS, and incubated for $1 \mathrm{~h}$ with DPBS with $\mathrm{Ca}^{2+}$ and $\mathrm{Mg}^{2+}$, supplemented with $0.2 \%$ BSA, containing FLUAV A/PR/8/34 at an MOI 0.5. Thereafter, the cells were washed with PBS and fresh infection medium was added. At $24 \mathrm{~h}$ post infection, the cells were fixed with ice cold methanol, blocked with $3 \%$ BSA for $1 \mathrm{~h}$, and then stained with mouse anti-myc and rabbit anti-PR8 HA antibodies (Biomol and Sino Biological, respectively). After $1 \mathrm{~h}$ of incubation with primary antibodies, the cells were washed three times with PBS and incubated for $1 \mathrm{~h}$ with anti-mouse and anti-rabbit secondary antibodies, coupled to Rhodamine Red-X and FITC (Dianova), respectively. After three final washing steps, the cells were incubated with Vectashield mounting medium (Vector Laboratories) and analyzed with a Zeiss LSM 5 laser scanning microscope. Image capture was 
performed with Pascal Software (Zeiss) and Fiji software was used for image analysis and calculation of Pearson Correlation Coefficient.

TTSP-mediated activation of CoV-S proteins for virus-cell fusion. For the analysis of the activation of SARS-S and MERS-S for virus-cell fusion, we employed a previously described retroviral pseudotyping system (35). Briefly, 293T cells were seeded in a T25 cell culture flask at a density of $7.0 \times 10^{5}$ cells/flask, cultured for $24 \mathrm{~h}$ and then transfected via calcium phosphate precipitation with plasmids encoding for MLV gag-pol $(3 \mu \mathrm{g})$, a firefly-luciferase harboring MLV vector $(6 \mu \mathrm{g})$, and a plasmid encoding for SARS-S or MERS-S $(3 \mu \mathrm{g})$. The transfection medium was replaced by fresh DMEM after overnight incubation and vector-containing supernatants were harvested at $48 \mathrm{~h}$ post transfection. Supernatants were passed through a $0.45 \mu \mathrm{m}$ filter, aliquoted and stored at $-80^{\circ} \mathrm{C}$. For analysis of SARS-S- and MERS-S-mediated entry, 293T cells were transiently cotransfected with plasmids encoding the SARS-CoV receptor ACE2 $(2 \mu \mathrm{g})$ or the MERS-CoV receptor CD26 $(2 \mu \mathrm{g})$ and the indicated proteases $(0.3 \mu \mathrm{g})$ or an empty plasmid as a control. The transfection medium was changed after overnight incubation, the cells were resuspended in fresh DMEM and seeded into 96-well plates. At $48 \mathrm{~h}$ post transfection, the cells were preincubated with dimethyl sulfoxide (DMSO) or $10 \mu \mathrm{M}$ cathepsin B and L inhibitor MDL 28170 (Calbiochem) for $1 \mathrm{~h}$ and then incubated with $50 \mu 1$ of infectivity-normalized pseudotypes for $8 \mathrm{~h}$. Thereafter, $50 \mu \mathrm{l}$ of fresh medium was added and the luciferase activities in cell lysate were determined at $72 \mathrm{~h}$ post transduction using a commercially available kit (Beetle Juice (PJK)). 


\section{RESULTS}

\section{TMPRSS2/TMPRSS3 chimeras are efficiently expressed and autocatalytically activated in 293T}

cells. In order to determine which domains in TTSPs are required for HA proteolytic processing, we constructed chimeras between TMPRSS2 (which activates HA) and TMPRSS3 (which fails to activate HA) by exchanging the cytoplasmic domain, cytoplasmic and transmembrane domain or cytoplasmic, transmembrane and stem domain (Fig 1A). All wild type (wt) and chimeric proteins were efficiently expressed in $293 \mathrm{~T}$ cells and exhibited the expected molecular weights (Fig 1B). Moreover, signals for the zymogen form and/or the activated form (protease domain proteolytically separated from the stem domain) were detected for each protease (Fig 1B), although the degree of activation varied between the chimeras tested. Thus, domain exchange between TMPRSS2 and TMPRSS3 is compatible with robust expression and autocatalytic activation.

\section{Cleavage and activation of FLUAV-HA and CoV S proteins by TMPRSS2/TMPRSS3 chimeras.} We next assessed whether the TMPRSS2/TMPRSS3 chimeras were able to cleave and activate FLUAV HA. To assess HA cleavage, we coexpressed proteases and HA of the H1 subtype and analyzed HA processing by Western blot. The presence of the HA1 subunit, which indicates processing of the HA0 precursor, was observed when cells coexpressed TMPRSS2 wt or were treated with trypsin (Fig 2A), as expected. Additionally, HA1 was detected when HA and chimeras 3-2-2-2, 3-3-2-2 and 2-2-2-3 were coexpressed (Fig 2A). In contrast, TMPRSS3 wt and chimeras 3-3-3-2, 2-33-3 and 2-2-3-3 failed to process HA. In order to assess whether cleavage resulted in activation, the protease expressing cells were infected with FLUAV A/PuertoRico/8/34 (H1N1) or A/Panama/2007/99 (H3N2) and release of infectious units in culture supernatants was determined. TMPRSS2 wt and trypsin treatment facilitated viral spread (Fig 2B,C), as expected. In addition, all chimeras able to cleave HA also promoted viral spread while the remaining chimeras were inactive (Fig 2B,C). Next, we asked whether cleavage and activation of FLUAV HA reflected that of SARS-S 
and MERS-S, which are also substrates of TMPRSS2 (20-24). Indeed, TMPRSS2 wt and chimeras 32-2-2, 3-3-2-2 and 2-2-2-3, which were able to process HA, also cleaved and activated SARS-S for cathepsin L-independent virus-cell entry, and comparable observations were made for MERS-S (Fig 3). Inspection of the chimeras which were able to activate FLUAV-HA and CoV S proteins revealed that all contained the TMPRSS2 stem domain, suggesting that this domain is an important determinant of HA and S protein cleavage and activation by TMPRSS2.

The TMPRSS2 stem domain controls HA cleavage and activation. In order to provide formal proof that the stem region of TMPRSS2 controls HA cleavage and activation, we constructed a chimera in which the cytoplasmic, transmembrane and catalytic domain originated from TMPRSS3 while the stem region was derived from TMPRSS2 (chimera 3-3-2-3, Fig 4A). The resulting protein was robustly expressed in transfected $293 \mathrm{~T}$ cells and was activated, since bands with the molecular weight expected for the zymogen and for the $\mathrm{N}$-terminal fragment produced upon activation were observed (Fig 4B). Importantly, chimera 3-3-2-3 cleaved (Fig 3C) and activated HA (Fig 3D), demonstrating that the TMPRSS2 stem domain is a key determinant of HA cleavage and activation.

The TMPRSS4 stem domain controls HA cleavage and activation. To confirm that our findings made for TMPRSS2 are also applicable to other members of the TMPRSS/hepsin subfamily of TTSPs, we constructed chimeras between TMPRSS4 (which activates FLUAV (27)) and TMPRSS3 (which fails to activate FLUAV), in which the cytoplasmic, transmembrane and stem domains were exchanged, resulting in constructs 4-4-4-3 and 3-3-3-4 (Fig 5A). Western blot analysis demonstrated that all proteases analyzed were robustly expressed (Fig 5B). For TMPRSS4 and 4-4-4-3 mainly the zymogen forms were detected (with the expected molecular weights of 55 and $53 \mathrm{kDa}$, respectively) while proteolytic processing products predominated in cells expressing TMPRSS3 and 3-3-3-4 (with the expected molecular weights of 37 and $37 \mathrm{kDa}$, respectively) (Fig 5B). TMPRSS4 expression and trypsin treatment resulted in the robust production of the HA1 subunit in transfected cells (Fig 5C), as 
expected, and the same observation was made for chimera 4-4-4-3. In contrast, chimera 3-3-3-4 was inactive (Fig 5C). Comparable results were obtained when activation of FLUAV and MERS-S was analyzed: TMPRSS4 wt and chimera 4-4-4-3 activated H1N1 (Fig 5D) and H3N2 FLUAV (Fig 5E) as well as MERS-S (Fig 5F) for viral spread and S protein-mediated transduction, respectively, while TMPRSS3 wt and chimera 3-3-3-4 were inactive. These results demonstrate that the stem domain of TMPRSS4 and likely other TTSPs is a central determinant of HA and CoV-S protein activation.

The TMPRSS2 stem region determines protease colocalization with HA. The unexpected finding that the stem domain (and not the protease domain) controls cleavage and activation of viral glycoproteins raised questions regarding the underlying mechanism. We had previously demonstrated that HA-activating TTSPs (i.e. TMPRSS2, DESC1 and MSPL) colocalized with HA at or near to the plasma membrane while an inactive TTSP (i.e. TMPRSS3) did not colocalize with HA (28). Therefore, we asked whether the TMPRSS2 stem region controls the ability of TMPRSS2/TMPRSS3 chimeras to activate HA by determining their ability to colocalize with HA. To this end, we expressed TMPRSS2, TMPRSS3, 3-3-3-2 and 2-2-2-3 in COS-7 cells, infected the cells with FLUAV and employed immunostaining and confocal microscopy to assess colocalization of HA and proteases. We found that TMPRSS2 and the HA-activating chimera 2-2-2-3 were present at or near to the cellular surface (Fig 6A) and colocalized with FLUAV HA, as demonstrated by a Pearson Correlation Coefficient above 0.6 (Fig 6B). In contrast, TMPRSS3 wt and chimera 3-3-3-2, which failed to cleave HA, demonstrated a predominantly cytoplasmic localization, and did not colocalize appreciably with HA (Fig 6A,B). These results suggest that the stem regions of TMPRSS2 and TMPRSS3 control cleavage (or absence thereof) of viral glycoproteins by determining the cellular localization of these proteases.

Dextran sulphate suppresses HA activation by TTSPs harboring a scavenger receptor A domain. The important role of the stem domain in virus activation raised the question whether this 
domain is a potential target for antiviral intervention. The stem region of HA activating enzymes of the TTSP/Hepsin subfamily, TMPRSS2, TMPRSS4 and hepsin, contains a scavenger receptor A (SRA) domain which exhibits up to $60 \%$ sequence homology with SR-A (Fig 7A). An antibody targeting the SR-A domain in TTSPs was not available but dextran sulphate was previously shown to block ligand binding to SR-A $(29,30)$. Therefore, we asked whether dextran sulphate blocks FLUAV spread in cells expressing HA-activating TTSPs containing an SR-A domain. As negative control, we employed chondroitin sulphate, which does not interfere with ligand binding to SR-A (36). Moreover, cells transfected with DESC1 and HAT - members of the HAT/DESC subfamily of TTSPs, which can activate HA but do not contain an SR-A domain - were used as additional negative controls. FLUAV infection of the transfected cells revealed that TTSP expression or addition of trypsin to the culture medium activated FLUAV for robust spread while spread in cells transfected with empty plasmid was inefficient (Fig 7B), as expected. Treatment of cells with chondroitin sulphate before addition of virus did not alter viral spread irrespective of the presence of protease. Similarly, dextran sulphate did not alter spread in cells transfected with empty plasmid and treated with PBS or trypsin or in cells expressing DESC1 and HAT. In contrast, dextran sulphate treatment markedly reduced viral spread in cells expressing HA-activating TTSPs containing a SR-A domain (Fig 7B), TMPRSS2, TMPRSS4, MSPL and hepsin.

We next tested whether dextran sulphate inhibits FLULAV spread in Caco-2 and Calu-3 cells, which endogenously express TMPRSS2 and thus allow trypsin-independent viral spread $(12,13,37)$. Dextran sulphate efficiently and dose-dependently reduced viral spread in these cells while chondroitin sulphate had not effect (Fig 7C), in keeping with the concept that antiviral activity of dextran sulphate may result from blockade of TMPRSS2 or related proteases. In order to obtain direct proof for this mechanism of action, we coexpressed HA and TMPRSS4 (contains the SR-A domain) or DESC1 (does not contain the SR-A domain) and analyzed HA cleavage by Western blot. Both proteases were chosen because their expression results in particularly robust HA cleavage in transfected cells. In the absence of protease, only uncleaved HA0 was detected irrespective of the presence or absence of chondroitin or dextran sulphate (Fig 7D), in keeping with expectations. 
Moreover, coexpression of TMPRSS4 and DESC1 resulted in almost complete processing of HA0 into HA01 (Fig 7D) and HA2 (not detected), again in keeping with expectations, and processing efficiency was not altered by treatment of cells with chondroitin sulphate (Fig 7D). In contrast, HA cleavage by TMPRSS4 was reproducibly and significantly reduced by dextran sulphate while HA processing by DESC1 was increased in the presence of this compound (Fig 7D), demonstrating that dextran sulphate selectively inhibits HA processing by TTSPs harboring an SR-A domain. 


\section{DISCUSSION}

TTSPs are plasma membrane-associated serine proteases that process a range of cellular substrates, including growth factors and components of the extracellular matrix $(38,39)$. The observation that aberrant expression of TTSPs is associated with several cancers moved these enzymes into the center of efforts to identify tumor markers and anti-tumor therapies $(38,39)$. More recently, it was discovered that the glycoproteins of FLUAV and other viruses are substrates of TTSPs $(8,9,40)$, indicating that these proteases are also potential targets for antiviral intervention. The success of such endeavors depends, at least in part, on our understanding of the determinants controlling ligand specificity of TTSPs, which are largely unclear. Here, we show that the stem region determines whether TTSPs can process viral glycoproteins. Moreover, our study provides evidence that the SRA domain present in the stem of certain TTSPs is a potential target for inhibitors. These findings not only provide important insights into ligand recognition by TTSPs but might also afford new opportunities to target TTSPs for therapeutic intervention.

The TTSP family contains more than 20 members which are grouped into four subfamilies, Hepsin/TMPRSS, Matriptase, Corin and HAT/DESC $(38,39)$. TTSPs which activate HA in cell culture are found in the TMPRSS/hepsin family (TMPRSS2, TMPRSS4, hepsin and MSPL, $(12,14$, 28)), the Matriptase subfamily (matriptase (41-43)) and the HAT/DESC family (HAT, DESC1 (14, 28)). TMPRSS2 activates diverse viruses in cell culture and expression of the protease in rodents is critical for spread and pathogenesis of FLUAV (16-18) and potentially also SARS-CoV (44). Nevertheless, other TTSPs might contribute to viral activation under certain conditions. Thus, several members of the TTSP family were found to activate viruses in cell culture $(14,27,28,41-43)$ and these enzymes might contribute to the activation of recently identified viruses with reduced or absent TMPRSS2-dependence $(16,45)$. In contrast, a panel of other TTSPs was found to be unable to activate viruses, despite robust expression in cultured cells (28), raising the question which properties of TTSPs determine specificity for viral glycoproteins. 
We employed chimeric TTSPs to identify determinants of ligand recognition. The stepwise substitution of the cytoplasmic, transmembrane and stem domain of TMPRSS2 by the corresponding domains of TMPRSS3 and vice versa pointed towards a key role of the stem in HA cleavage and activation. This conclusion was further supported by the analysis of TMPRSS3/TMPRSS4 chimeras and by the demonstration that replacing the TMPRSS3 stem by that of TMPRSS2 is sufficient to render the chimeric enzyme capable of HA cleavage and activation. How is the stem region organized and how does it impact ligand recognition? The stem region of TTSPs can harbor up to 11 domains derived from 6 proteins, including SR-A, and is known to impact zymogen activation and sensitivity of TTSPs to protease inhibitors $(38,39)$. Moreover, a role for the stem region in the proteolytic processing of ligands has been reported: a frizzled 1 domain and LDLR repeats with the stem region of corin were shown to be essential for processing of a physiological substrate, pro-ANP (46), while the stem region of enteropeptidase was found to be required for processing of macromolecules but not small peptides (47). However, the underlying mechanism remained unclear.

It is conceivable that the stem region impacts ligand recognition by ensuring the adequate spatial presentation of the catalytic domain and such a structural role has been proposed for the stem of corin (46). Our study suggests a second possibility: The stem might determine whether a TTSP comes into contact with a potential ligand by controlling the cellular localization of the enzyme. Thus, the stem region of FLUAV activating TTSPs was found to be required for colocalization with HA at or close to the cell surface, the site where HA is believed to be activated by TTSPs. This finding is in line with our previous observation that the ability of TTSPs to activate HA correlates with their capacity to colocalize with this protein (28). Our observations are also in keeping with the concept that the stem might impact TTSP trafficking within cells, which has been posited in review articles $(38,39)$, but for which, to our knowledge, direct experimental evidence is missing. In sum, the stem determines whether TTSPs colocalize with HA and this raises the question whether this domain is a target for inhibitors.

The stem regions of TMPRSS2 and TMPRSS3 contain a single protein domain, which exhibits 58 and $61 \%$, respectively, homology with the scavenger receptor cysteine-rich domain 
(SRCR) of SR-A. The SRCR facilitates binding of SR-A to a variety of modified proteins and lipids of cellular origin as well as to pathogen-associated molecular patterns (PAMPs) and clears these ligands from the circulation (48). Dextran sulfate, a polyanion, is a well-established inhibitor of ligand binding to SR-A $(29,30)$ and was therefore used to probe whether targeting the SR-A domain in TTSPs inhibits FLUAV activation. Indeed, dextran sulfate treatment selectively inhibited FLUAV activation by TTSPs containing a SR-A domain while chondroitin sulfate, which fails to inhibit ligand binding to SR-A (36), did not exert inhibitory activity. In addition, dextran sulfate modestly reduced HA processing by TMPRSS4 (which contains an SR-A domain) but not DESC1 (which does not contain an SR-A domain) in HA transfected cells, indicating that inhibition of FLUAV spread by dextran sulfate was due to blockade of HA activation. In this context, it should be noted that treatment of cells with camostat mesylate, which blocks activation of viruses by TTSPs with high efficiency $(20,23,44,49,50)$, also had only moderate effects on HA cleavage in transfected cells (data not shown). Thus, the very high levels of HA and TTSP expression in transfected 293T cells may preclude visualizing efficient inhibition of HA cleavage. Finally, it is noteworthy that dextran sulphate was previously shown to inhibit A/PR/8/34 spread in MDCK cells in an NA-dependent fashion (51). Why similar effects were not observed in the present study (conduced with 293T cells) is at present unclear, and cell type specific differences might be accountable. In sum, we obtained evidence that dextran sulphate can block TTSP-dependent HA activation by targeting the SR-A domain within the stem of certain TTSPs.

Collectively, our study identifies the TMPRSS2 stem as the key determinant of activation of viral glycoproteins and as a potential target for intervention. Evidence is accumulating that TMPRSS2 is essential for spread of respiratory viruses and inhibitors which target TMPRSS2 along with many other proteases have been shown to display antiviral activity in the host $(52,53)$. The goal of future research endeavors should thus be the development of inhibitors specific for TMPRSS2 and the discovery of the stem region as a target for intervention should aid these efforts. 


\section{ACKNOWLEDGEMENTS}

We thank S. Bertram for valuable discussion and DFG (PO 716/6-1) and the Leibniz Association (Graduate School Emerging Infectious Diseases) for funding. This work was partly supported by the Göttingen Graduate School for Neurosciences, Biophysics and Molecular Biosciences (DFG grant GSC 226/1 and GSC 226/2). 


\section{REFERENCES}

1. Molinari, N. A., I. R. Ortega-Sanchez, M. L. Messonnier, W. W. Thompson, P. M. Wortley, E. Weintraub, and C. B. Bridges. 2007. The annual impact of seasonal influenza in the US: measuring disease burden and costs. Vaccine 25:5086-5096.

2. WHO. Influenza (Seasonal). http://www.who.int/mediacentre/factsheets/fs211/en/

3. Johnson, N. P. and J. Mueller. 2002. Updating the accounts: global mortality of the 19181920 "Spanish" influenza pandemic. Bull. Hist Med. 76:105-115.

4. Krammer, F. and P. Palese. 2015. Advances in the development of influenza virus vaccines. Nat. Rev. Drug Discov. 14:167-182.

5. Loregian, A., B. Mercorelli, G. Nannetti, C. Compagnin, and G. Palu. 2014. Antiviral strategies against influenza virus: towards new therapeutic approaches. Cell Mol. Life Sci. 71:3659-3683.

6. Hurt, A. C. 2014. The epidemiology and spread of drug resistant human influenza viruses. Curr. Opin. Virol. 8:22-29.

7. Edinger, T. O., M. O. Pohl, and S. Stertz. 2014. Entry of influenza A virus: host factors and antiviral targets. J. Gen. Virol. 95:263-277.

8. Bertram, S., I. Glowacka, I. Steffen, A. Kuhl, and S. Pohlmann. 2010. Novel insights into proteolytic cleavage of influenza virus hemagglutinin. Rev. Med. Virol. 20:298-310.

9. Bottcher-Friebertshauser, E., H. D. Klenk, and W. Garten. 2013. Activation of influenza viruses by proteases from host cells and bacteria in the human airway epithelium. Pathog. Dis. 69:87-100.

10. Kido, H., Y. Yokogoshi, K. Sakai, M. Tashiro, Y. Kishino, A. Fukutomi, and N.

Katunuma. 1992. Isolation and characterization of a novel trypsin-like protease found in rat bronchiolar epithelial Clara cells. A possible activator of the viral fusion glycoprotein. J. Biol. Chem. 267:13573-13579.

11. Kido, H., Y. Okumura, H. Yamada, T. Q. Le, and M. Yano. 2007. Proteases essential for human influenza virus entry into cells and their inhibitors as potential therapeutic agents. Curr. Pharm. Des 13:405-414.

12. Bertram, S., I. Glowacka, P. Blazejewska, E. Soilleux, P. Allen, S. Danisch, I. Steffen, S. Y. Choi, Y. Park, H. Schneider, K. Schughart, and S. Pohlmann. 2010. TMPRSS2 and TMPRSS4 facilitate trypsin-independent spread of influenza virus in Caco-2 cells. J. Virol. 84:10016-10025.

13. Bottcher-Friebertshauser, E., D. A. Stein, H. D. Klenk, and W. Garten. 2011. Inhibition of influenza virus infection in human airway cell cultures by an antisense peptide-conjugated morpholino oligomer targeting the hemagglutinin-activating protease TMPRSS2. J. Virol. 85:1554-1562. 
14. Bottcher, E., T. Matrosovich, M. Beyerle, H. D. Klenk, W. Garten, and M. Matrosovich. 2006. Proteolytic activation of influenza viruses by serine proteases TMPRSS2 and HAT from human airway epithelium. J. Virol. 80:9896-9898.

15. Bertram, S., A. Heurich, H. Lavender, S. Gierer, S. Danisch, P. Perin, J. M. Lucas, P. S. Nelson, S. Pohlmann, and E. J. Soilleux. 2012. Influenza and SARS-coronavirus activating proteases TMPRSS2 and HAT are expressed at multiple sites in human respiratory and gastrointestinal tracts. PLoS. One. 7:e35876.

16. Hatesuer, B., S. Bertram, N. Mehnert, M. M. Bahgat, P. S. Nelson, S. Pohlman, and K. Schughart. 2013. Tmprss2 Is Essential for Influenza H1N1 Virus Pathogenesis in Mice. PLoS. Pathog. 9:e1003774.

17. Sakai, K., Y. Ami, M. Tahara, T. Kubota, M. Anraku, M. Abe, N. Nakajima, T. Sekizuka, K. Shirato, Y. Suzaki, A. Ainai, Y. Nakatsu, K. Kanou, K. Nakamura, T. Suzuki, K. Komase, E. Nobusawa, K. Maenaka, M. Kuroda, H. Hasegawa, Y. Kawaoka, M. Tashiro, and M. Takeda. 2014. The Host Protease TMPRSS2 Plays a Major Role in In Vivo Replication of Emerging H7N9 and Seasonal Influenza Viruses. J. Virol. 88:5608-5616.

18. Tarnow, C., G. Engels, A. Arendt, F. Schwalm, H. Sediri, A. Preuss, P. S. Nelson, W. Garten, H. D. Klenk, G. Gabriel, and E. Bottcher-Friebertshauser. 2014. TMPRSS2 Is a Host Factor That Is Essential for Pneumotropism and Pathogenicity of H7N9 Influenza A Virus in Mice. J. Virol. 88:4744-4751.

19. Cheng, Z., J. Zhou, K. K. To, H. Chu, C. Li, D. Wang, D. Yang, S. Zheng, K. Hao, Y. Bosse, M. Obeidat, C. A. Brandsma, Y. Q. Song, Y. Chen, B. J. Zheng, L. Li, and K. Y. Yuen. 2015. Identification of TMPRSS2 as a Susceptibility Gene for Severe 2009 Pandemic A(H1N1) Influenza and A(H7N9) Influenza. J. Infect. Dis.

20. Gierer, S., S. Bertram, F. Kaup, F. Wrensch, A. Heurich, A. Kramer-Kuhl, K. Welsch, M. Winkler, B. Meyer, C. Drosten, U. Dittmer, H. T. von, G. Simmons, H. Hofmann, and S. Pohlmann. 2013. The spike protein of the emerging betacoronavirus EMC uses a novel coronavirus receptor for entry, can be activated by TMPRSS2, and is targeted by neutralizing antibodies. J. Virol. 87:5502-5511.

21. Glowacka, I., S. Bertram, M. A. Muller, P. Allen, E. Soilleux, S. Pfefferle, I. Steffen, T. S. Tsegaye, Y. He, K. Gnirss, D. Niemeyer, H. Schneider, C. Drosten, and S. Pohlmann 2011. Evidence that TMPRSS2 activates the severe acute respiratory syndrome coronavirus spike protein for membrane fusion and reduces viral control by the humoral immune response. J. Virol. 85:4122-4134.

22. Matsuyama, S., N. Nagata, K. Shirato, M. Kawase, M. Takeda, and F. Taguchi. 2010. Efficient activation of the severe acute respiratory syndrome coronavirus spike protein by the transmembrane protease TMPRSS2. J. Virol. 84:12658-12664.

23. Shirato, K., M. Kawase, and S. Matsuyama. 2013. Middle East respiratory syndrome coronavirus infection mediated by the transmembrane serine protease TMPRSS2. J. Virol. 87:12552-12561. 
24. Shulla, A., T. Heald-Sargent, G. Subramanya, J. Zhao, S. Perlman, and T. Gallagher. 2011. A transmembrane serine protease is linked to the severe acute respiratory syndrome coronavirus receptor and activates virus entry. J. Virol. 85:873-882.

25. Esumi, M., M. Ishibashi, H. Yamaguchi, S. Nakajima, Y. Tai, S. Kikuta, M. Sugitani, T. Takayama, M. Tahara, M. Takeda, and T. Wakita. 2015. Transmembrane serine protease TMPRSS2 activates hepatitis C virus infection. Hepatology 61:437-446.

26. Kim, T. S., C. Heinlein, R. C. Hackman, and P. S. Nelson. 2006. Phenotypic analysis of mice lacking the Tmprss2-encoded protease. Mol. Cell Biol. 26:965-975.

27. Chaipan, C., D. Kobasa, S. Bertram, I. Glowacka, I. Steffen, T. S. Tsegaye, M. Takeda, T. H. Bugge, S. Kim, Y. Park, A. Marzi, and S. Pohlmann. 2009. Proteolytic activation of the 1918 influenza virus hemagglutinin. J. Virol. 83:3200-3211.

28. Zmora, P., P. Blazejewska, A. S. Moldenhauer, K. Welsch, I. Nehlmeier, Q. Wu, H. Schneider, S. Pohlmann, and S. Bertram. 2014. DESC1 and MSPL activate influenza A viruses and emerging coronaviruses for host cell entry. J. Virol. 88:12087-12097.

29. Goldstein, J. L., Y. K. Ho, S. K. Basu, and M. S. Brown. 1979. Binding site on macrophages that mediates uptake and degradation of acetylated low density lipoprotein, producing massive cholesterol deposition. Proc. Natl. Acad. Sci. U. S. A 76:333-337.

30. Tsubamoto, Y., N. Yamada, Y. Watanabe, T. Inaba, M. Shiomi, H. Shimano, T. Gotoda, K. Harada, M. Shimada, J. Ohsuga, and . 1994. Dextran sulfate, a competitive inhibitor for scavenger receptor, prevents the progression of atherosclerosis in Watanabe heritable hyperlipidemic rabbits. Atherosclerosis 106:43-50.

31. Niwa, H., K. Yamamura, and J. Miyazaki. 1991. Efficient selection for high-expression transfectants with a novel eukaryotic vector. Gene 108:193-199.

32. Schindelin, J., I. Arganda-Carreras, E. Frise, V. Kaynig, M. Longair, T. Pietzsch, S. Preibisch, C. Rueden, S. Saalfeld, B. Schmid, J. Y. Tinevez, D. J. White, V. Hartenstein, K. Eliceiri, P. Tomancak, and A. Cardona. 2012. Fiji: an open-source platform for biological-image analysis. Nat. Methods 9:676-682.

33. Matthaei, M., M. Budt, and T. Wolff. 2013. Highly pathogenic H5N1 influenza A virus strains provoke heterogeneous IFN-alpha/beta responses that distinctively affect viral propagation in human cells. PLoS. One. 8: 56659.

34. Winkler, M., S. Bertram, K. Gnirss, I. Nehlmeier, A. Gawanbacht, F. Kirchhoff, C. Ehrhardt, S. Ludwig, M. Kiene, A. S. Moldenhauer, U. Goedecke, C. B. Karsten, A. Kuhl, and S. Pohlmann. 2012. Influenza A virus does not encode a tetherin antagonist with Vpu-like activity and induces IFN-dependent tetherin expression in infected cells. PLoS. One. 7:e43337.

35. Wrensch, F., M. Winkler, and S. Pohlmann. 2014. IFITM proteins inhibit entry driven by the MERS-coronavirus spike protein: evidence for cholesterol-independent mechanisms. Viruses. 6:3683-3698. 
36. Bowdish, D. M., K. Sakamoto, M. J. Kim, M. Kroos, S. Mukhopadhyay, C. A. Leifer, K. Tryggvason, S. Gordon, and D. G. Russell. 2009. MARCO, TLR2, and CD14 are required for macrophage cytokine responses to mycobacterial trehalose dimycolate and Mycobacterium tuberculosis. PLoS. Pathog. 5:e1000474.

37. Zhirnov, O. and H. D. Klenk. 2003. Human influenza A viruses are proteolytically activated and do not induce apoptosis in CACO-2 cells. Virology 313:198-212.

38. Antalis, T. M., T. H. Bugge, and Q. Wu. 2011. Membrane-anchored serine proteases in health and disease. Prog. Mol. Biol. Transl. Sci. 99:1-50.

39. Szabo, R. and T. H. Bugge. 2011. Membrane-anchored serine proteases in vertebrate cell and developmental biology. Annu. Rev. Cell Dev. Biol. 27:213-235.

40. Garten, W., C. Braden, A. Arendt, C. Peitsch, J. Baron, Y. Lu, K. Pawletko, K. Hardes, T. Steinmetzer, and E. Bottcher-Friebertshauser. 2015. Influenza virus activating host proteases: Identification, localization and inhibitors as potential therapeutics. Eur. J. Cell Biol.

41. Baron, J., C. Tarnow, D. Mayoli-Nussle, E. Schilling, D. Meyer, M. Hammami, F. Schwalm, T. Steinmetzer, Y. Guan, W. Garten, H. D. Klenk, and E. BottcherFriebertshauser. 2013. Matriptase, HAT, and TMPRSS2 activate the hemagglutinin of H9N2 influenza A viruses. J. Virol. 87:1811-1820.

42. Beaulieu, A., E. Gravel, A. Cloutier, I. Marois, E. Colombo, A. Desilets, C. Verreault, R. Leduc, E. Marsault, and M. V. Richter. 2013. Matriptase proteolytically activates influenza virus and promotes multicycle replication in the human airway epithelium. J. Virol. 87:42374251.

43. Hamilton, B. S., D. W. Gludish, and G. R. Whittaker. 2012. Cleavage activation of the human-adapted influenza virus subtypes by matriptase reveals both subtype and strain specificities. J. Virol. 86:10579-10586.

44. Zhou, Y., P. Vedantham, K. Lu, J. Agudelo, R. Carrion, Jr., J. W. Nunneley, D. Barnard, S. Pohlmann, J. H. McKerrow, A. R. Renslo, and G. Simmons. 2015. Protease inhibitors targeting coronavirus and filovirus entry. Antiviral Res. 116:76-84.

45. Sakai, K., T. Sekizuka, Y. Ami, N. Nakajima, M. Kitazawa, Y. Sato, K. Nakajima, M. Anraku, T. Kubota, K. Komase, K. Takehara, H. Hasegawa, T. Odagiri, M. Tashiro, M. Kuroda, and M. Takeda. 2015. A mutant H3N2 influenza virus uses an alternative activation mechanism in TMPRSS2 knockout mice by loss of an oligosaccharide in the hemagglutinin stalk region. J. Virol. 89:5154-5158.

46. Knappe, S., F. Wu, M. R. Madlansacay, and Q. Wu. 2004. Identification of domain structures in the propeptide of corin essential for the processing of proatrial natriuretic peptide. J. Biol. Chem. 279:34464-34471.

47. Lu, D., X. Yuan, X. Zheng, and J. E. Sadler. 1997. Bovine proenteropeptidase is activated by trypsin, and the specificity of enteropeptidase depends on the heavy chain. J. Biol. Chem. 272:31293-31300. 
48. Canton, J., D. Neculai, and S. Grinstein. 2013. Scavenger receptors in homeostasis and immunity. Nat. Rev. Immunol. 13:621-634.

49. Bertram, S., R. Dijkman, M. Habjan, A. Heurich, S. Gierer, I. Glowacka, K. Welsch, M. Winkler, H. Schneider, H. Hofmann-Winkler, V. Thiel, and S. Pohlmann. 2013.

TMPRSS2 activates the human coronavirus 229E for cathepsin-independent host cell entry and is expressed in viral target cells in the respiratory epithelium. J. Virol. 87:6150-6160.

50. Zhirnov, O. P., H. D. Klenk, and P. F. Wright. 2011. Aprotinin and similar protease inhibitors as drugs against influenza. Antiviral Res. 92:27-36.

51. Yamada, H., E. Moriishi, A. M. Haredy, N. Takenaka, Y. Mori, K. Yamanishi, and S. Okamoto. 2012. Influenza virus neuraminidase contributes to the dextran sulfate-dependent suppressive replication of some influenza A virus strains. Antiviral Res. 96:344-352.

52. Hosoya, M., S. Matsuyama, M. Baba, H. Suzuki, and S. Shigeta. 1992. Effects of protease inhibitors on replication of various myxoviruses. Antimicrob. Agents Chemother. 36:14321436.

53. Lee, M. G., K. H. Kim, K. Y. Park, and J. S. Kim. 1996. Evaluation of anti-influenza effects of camostat in mice infected with non-adapted human influenza viruses. Arch. Virol. 141:1979-1989. 


\section{Figure legends}

FIG 1 Expression and autocatalytic activation of TMPRSS2/TMPRSS3 chimeras. (A) Schematic representation of the chimeras between TMPRSS2 and TMPRSS3, which were analyzed in the present study. (B) Plasmids encoding the indicated proteases equipped with an N-terminal myc-tag were transfected into $293 \mathrm{~T}$ cells. Transfection of empty plasmid (pCAGGS) served as negative control. Protease expression in cell lysates was detected by Western blot using anti-myc antibody. Detection of $\beta$-actin served as loading control. Similar results were obtained in three separate experiments. Black-filled arrowheads indicate zymogen forms, while grey-filled arrowheads highlight cleavage products resulting from proteolytic activation.

FIG 2 The chimera 2-2-2-3 cleaves and activates the FLUAV hemagglutinin. (A) Expression plasmids encoding the FLUAV HA subtype $\mathrm{H} 1$ and the indicated proteases or empty plasmid (pCAGGS) were cotransfected into $293 \mathrm{~T}$ cells. The cells were then treated with PBS or trypsin and HA cleavage was detected by Western blot analysis of cell lysates. Arrows indicate the HA0 (upper arrow) and the HA1 (lower arrow) subunits. Detection of $\beta$-actin served as loading control. Similar results were obtained in three independent experiments. (B) The indicated proteases were expressed in $293 \mathrm{~T}$ cells and the cells were infected with FLUAV A/PR/8/34 (H1N1) at an MOI of 0.01 or FLUAV A/Panama/2007/1999 (H3N2) at an MOI of 0.1 and either treated with trypsin or PBS. At 48 h post infection the number of infectious units into the culture supernatants was quantified employed a focus formation assay. The results of representative experiments performed with triplicate samples are shown. Error bars indicate standard deviations. Similar results were obtained in three independent experiments. ffu, focus forming units. 
FIG 3 The 2-2-2-3 chimera cleaves and activates SARS-S and MERS-S. (A) In brief, 293T effector cells were transfected with either empty plasmid (pCAGGS) or pCAGGS encoding SARS-S. In parallel, 293T target cells were transfected with empty plasmid (pCAGGS) or plasmids encoding ACE2 and the indicated proteases. Effector and target cells were mixed, treated with PBS or trypsin, and SARS-S cleavage was analyzed by Western blotting with a V5-specific antibody. The expression of $\beta$-actin served as a loading control. The results are representative of three independent experiments with different plasmid preparations. Black-filled arrowhead, uncleaved SARS-S; gray-filled arrowhead, S2 subunit generated by trypsin digest; white-filled arrowheads, C-terminal cleavage fragments generated by TMPRSS2. (B) To analyze activation of SARS-S and MERS-S for virus-cell fusion, 293T cells were transiently transfected with plasmids encoding the indicated proteases and ACE2 or DPP4, respectively. At $24 \mathrm{~h}$ post transfection, the cells were pretreated with medium supplemented with DMSO or $10 \mu \mathrm{M}$ cathepsin $\mathrm{B} / \mathrm{L}$ inhibitor MDL 28170, and transduced with pseudotypes bearing SARS-S or MERS-S. The luciferase activities in cell lysates were analyzed at $72 \mathrm{~h}$ post transduction. The results of representative experiments performed with triplicate samples are shown. Error bars indicate standard deviations. Similar results were obtained in two separate experiments. c.p.s., counts per second.

FIG 4 The TMPRSS2 stem region determines HA cleavage. (A) Schematic representation of the chimeric protease analyzed. (B) Plasmids encoding TMPRSS2, TMPRSS3 and chimera3-3-2-3 equipped with an N-terminal myc tag were transfected into 293T cells. Empty plasmid (pCAGGS) served as a negative control. Protease expression in cell lysates was detected via Western blotting with anti-myc antibody. The results of a representative experiment are shown and were confirmed in three separate experiments. Expression of $\beta$-actin served as a loading control. (C) 293T cells were cotransfected with plasmids encoding FLUAV HA of subtype H1 and chimera 3-3-2-3 or empty plasmid (pCAGGS) and then treated with PBS or trypsin. At $48 \mathrm{~h}$ post transfection the HA cleavage in the cell lysate was analyzed by Western blotting. Detection of $\beta$-actin served as loading control. Similar results were obtained in three independent experiments. (D) The indicated proteases were 
transiently expressed in $293 \mathrm{~T}$ cells and the cells were infected with FLUAV A/PR/8/34 (H1N1) at an MOI 0.01 (left) or FLUAV A/Panama/2007/1999 at an MOI of 0.1 (right) and treated with either trypsin or PBS. At $48 \mathrm{~h}$ post infection, the virus release was measured by determination of infectious particles in the culture supernatant. The results of representative experiments performed with triplicate samples are shown. Error bars indicate standard deviations. Similar results were obtained in three independent experiments. ffu, focus forming units.

FIG 5 The stem region of TMPRSS4 determines HA cleavage and activation. (A) Schematic representation of the chimeric proteases analyzed. (B) $293 \mathrm{~T}$ cells were transiently transfected with plasmids encoding the indicated proteases equipped with an N-terminal myc tag or with empty plasmid (pCAGGS) as negative control. Protease expression in cell lysates was detected via Western blotting with anti-myc antibody. The expression of $\beta$-actin served as a loading control. Similar results were obtained in three separate experiments. (C) $293 \mathrm{~T}$ cells were cotransfected with plasmids encoding the FLUAV HA of subtype H1 and the indicated proteases or empty plasmid (pCAGGS) and then treated with PBS or trypsin. At $48 \mathrm{~h}$ post transfection the HA cleavage in cell lysate was analyzed via Western blotting. Detection of $\beta$-actin served as loading control. Similar results were obtained in three independent experiments. (D) The indicated proteases were expressed in 293T cells and the cells were infected with FLUAV A/PR/8/34 (H1N1) at an MOI 0.01 and treated with either trypsin or PBS. At $48 \mathrm{~h}$ post infection, the number of infectious units present in culture supernatants was determined using the focus formation assay. The results of representative experiments performed with triplicate samples are shown. Error bars indicate standard deviations. Similar results were obtained in three independent experiments. (E) The experiment was performed as described for panel (D) but the cells were infected with FLUAV A/Panama/2007/1999 at an MOI of 0.1. (F) To analyze activation of MERS-S for virus-cell fusion, 293T cells were transfected with plasmids encoding the indicated proteases and DPP4. At $24 \mathrm{~h}$ post transfection, the cells were pretreated with medium supplemented with DMSO or $10 \mu \mathrm{M}$ cathepsin B/L inhibitor MDL 28170 and transduced with MLV pseudotypes bearing MERS-S. At $72 \mathrm{~h}$ post transduction, the luciferase activities in cell lysates were 
measured. The results of a representative experiment performed with triplicate samples are shown and were confirmed in three separate experiments. Error bars indicate standard deviations. ffu, focus forming units; c.p.s., counts per second.

FIG 6 The stem region of TMPRSS2 determines colocalization with HA. (A) Plasmids encoding the indicated proteases were transfected into COS-7 cells. Subsequently, the cells were infected with FLUAV A/PR/8/34 (H1N1) at an MOI 0.5. At $24 \mathrm{~h}$ post infection, the cells were fixed with ice-cold methanol and stained for FLUAV-HA (green) and proteases (red). White squares show examples of colocalization of HA and proteases (yellow signals) which were digitally magnified by 4-fold. Similar results were obtained in three separate experiments. (B) The colocalization of proteases and HA was determined by analyzing images with Fiji software, which allows calculation of the Pearson Correlation Coefficient (PCC). The average PCC measured for 4-7 cells from separate experiments is shown, error bars indicate standard error of the mean (SEM).

FIG 7 Dextran sulphate inhibits activation of FLUAV HA by TTSPs containing a SR-A domain. (A) Alignment of the protein sequences of the SR-A domain in the TMPRSS2 stem region and the SRCR of SR-A. Red, small and hydrophobic: blue, acidic; magenta, basic; green, hydroxyl and sulfhydryl and amine. *, full conservation; :, strong conservation; ., weak conservation. (B) 293T cells were transfected with plasmids encoding indicated proteases and infected with FLUAV A/PR/8/34 (H1N1) at an MOI 0.01 and treated with either trypsin or PBS. After infection, the medium was replaced with fresh medium containing either PBS, $100 \mu \mathrm{g} / \mathrm{ml}$ dextran sulphate or $100 \mu \mathrm{g} / \mathrm{ml}$ chondroitin sulphate. At $48 \mathrm{~h}$ post infection, the infectious units present in culture supernatants were quantified. The infectious units measured in PBS treated cultures were set as 100\%. The average of three independent experiments carried out with triplicate samples is shown, error bars indicate SEM. The numbers above the white bars indicate the fold increase in viral titers relative to pCAGGS transfected cells. (C) Caco2 (left) or Calu-3 (right) cells were infected with FLUAV A/PR/8/34 (H1N1) at an MOI 0.01 and 
treated with increasing amount of dextran sulphate or chondroitin sulphate. The release of infectious units in culture supernatants was analyzed at $48 \mathrm{~h}$ post infection using a focus formation assay. The results of representative experiments performed with triplicate samples are shown. Error bars indicate standard deviations. (D) $293 \mathrm{~T}$ cells were cotransfected with plasmid encoding FLUAV HA and the indicated proteases and treated with PBS, $100 \mu \mathrm{g} / \mathrm{ml}$ dextran sulphate or $100 \mu \mathrm{g} / \mathrm{ml}$ chondroitin sulphate. At $48 \mathrm{~h}$ post transfection, the HA cleavage was determined by Western blotting. Similar results were obtained in three independent experiments. The $\mathrm{HA}_{0}$ (upper arrow) are $\mathrm{HA}_{1}$ (lower arrow) subunits are indicated. The expression of $\beta$-actin served as loading control. (E) Three Western blot experiments carried out as described for panel (D) were quantified using Fiji software. The pictures from three independent experiments were analyzed with Plot Lanes, and the sum of HA0 and HA1 signals was set as $100 \%$. Error bars indicate SEM. *, p $<0.05$ 


\section{Figure 1}
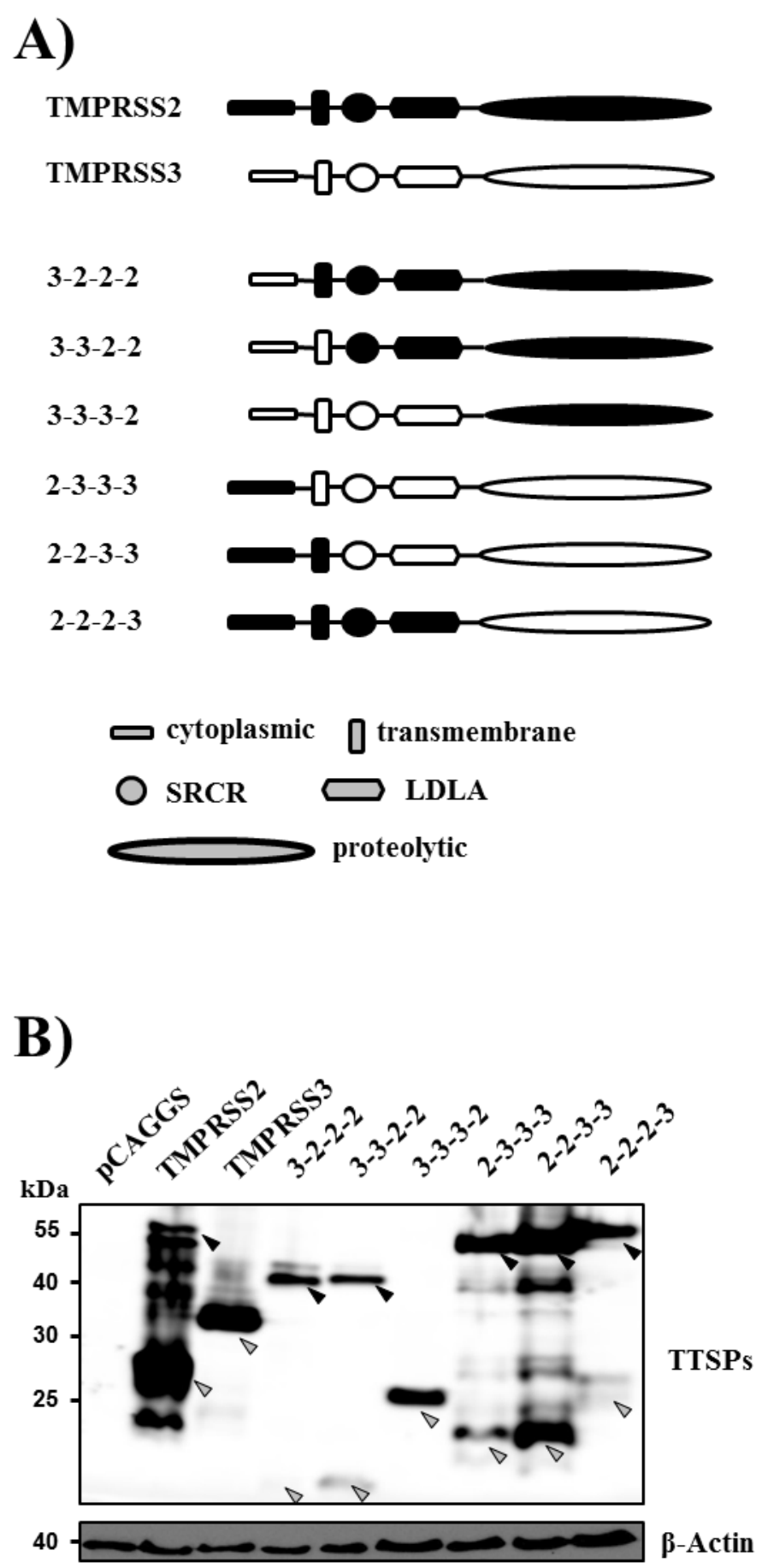
Figure 2

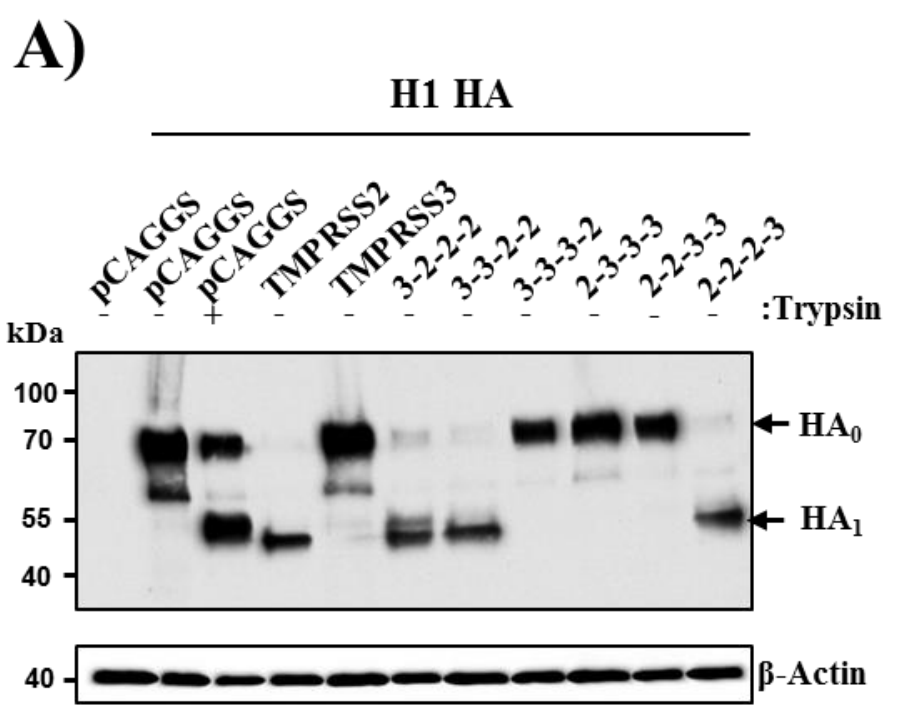

B)
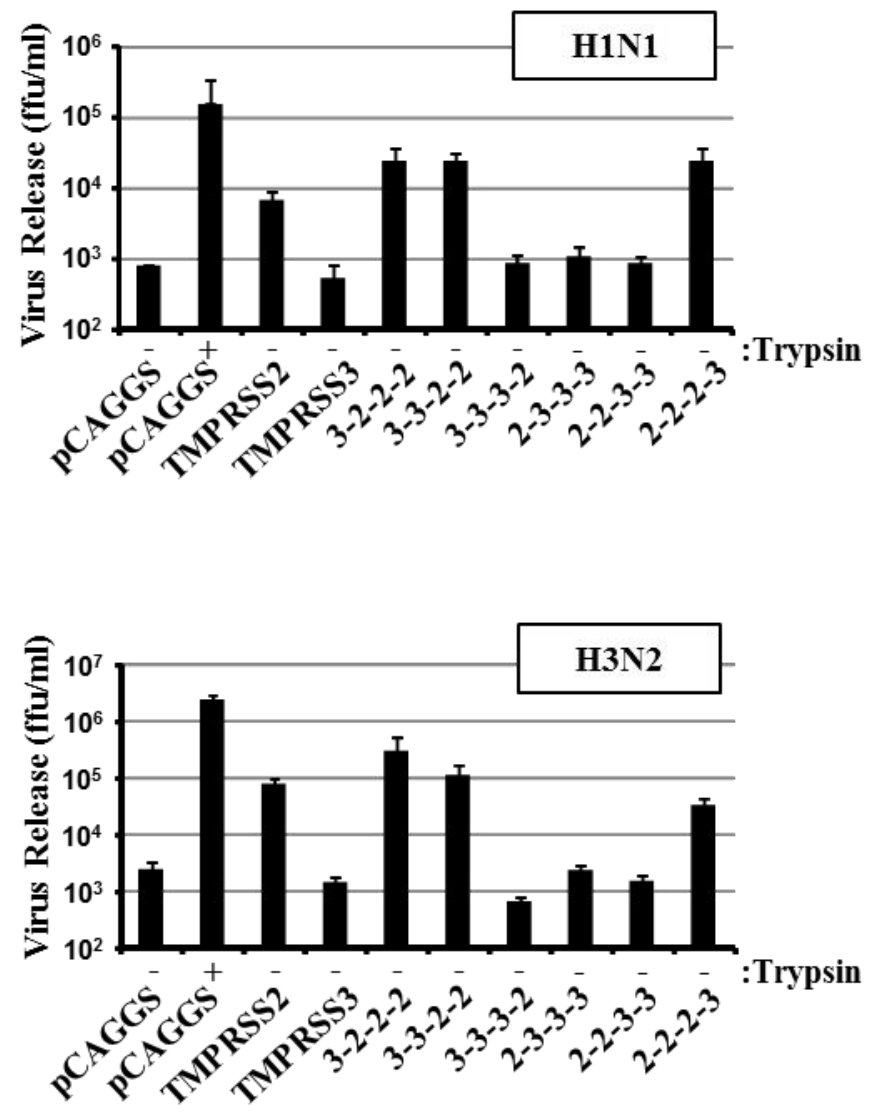
Figure 3

A)

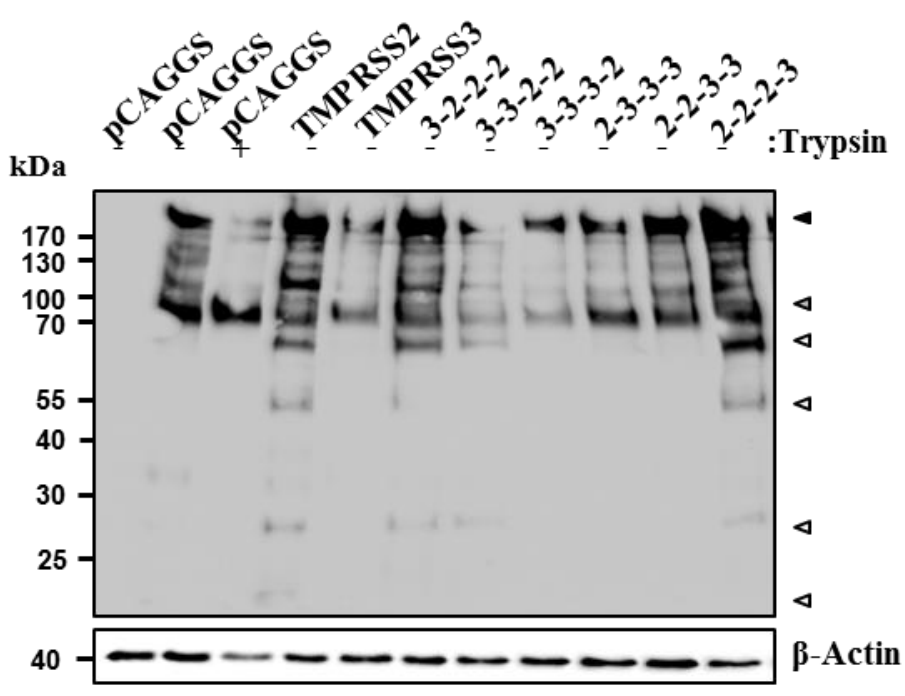

B)

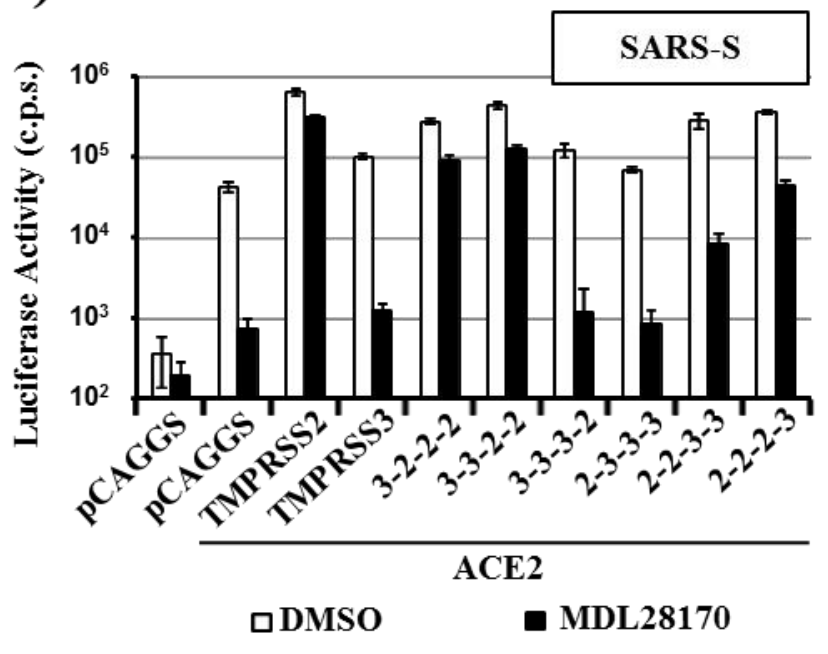

C)

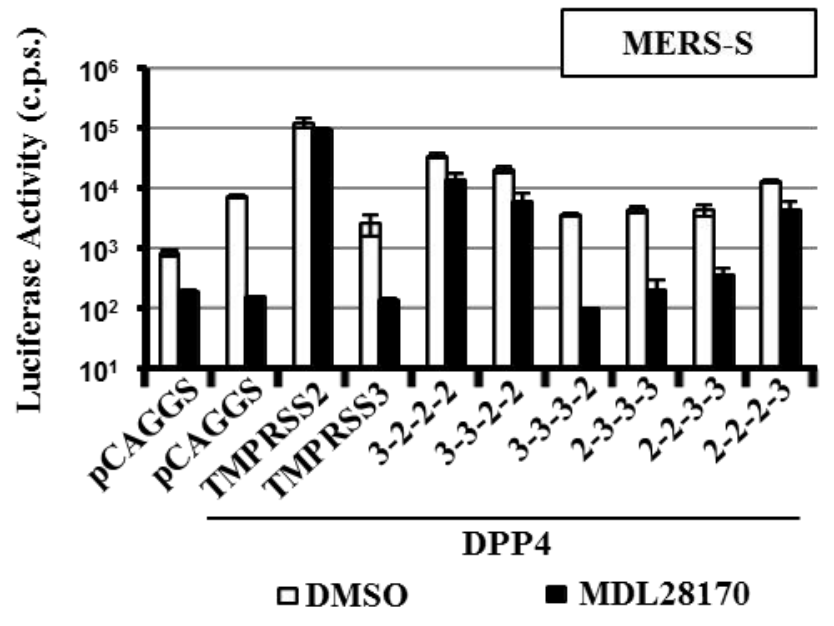


Figure 4

A)

3-3-2-3

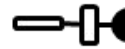

B)
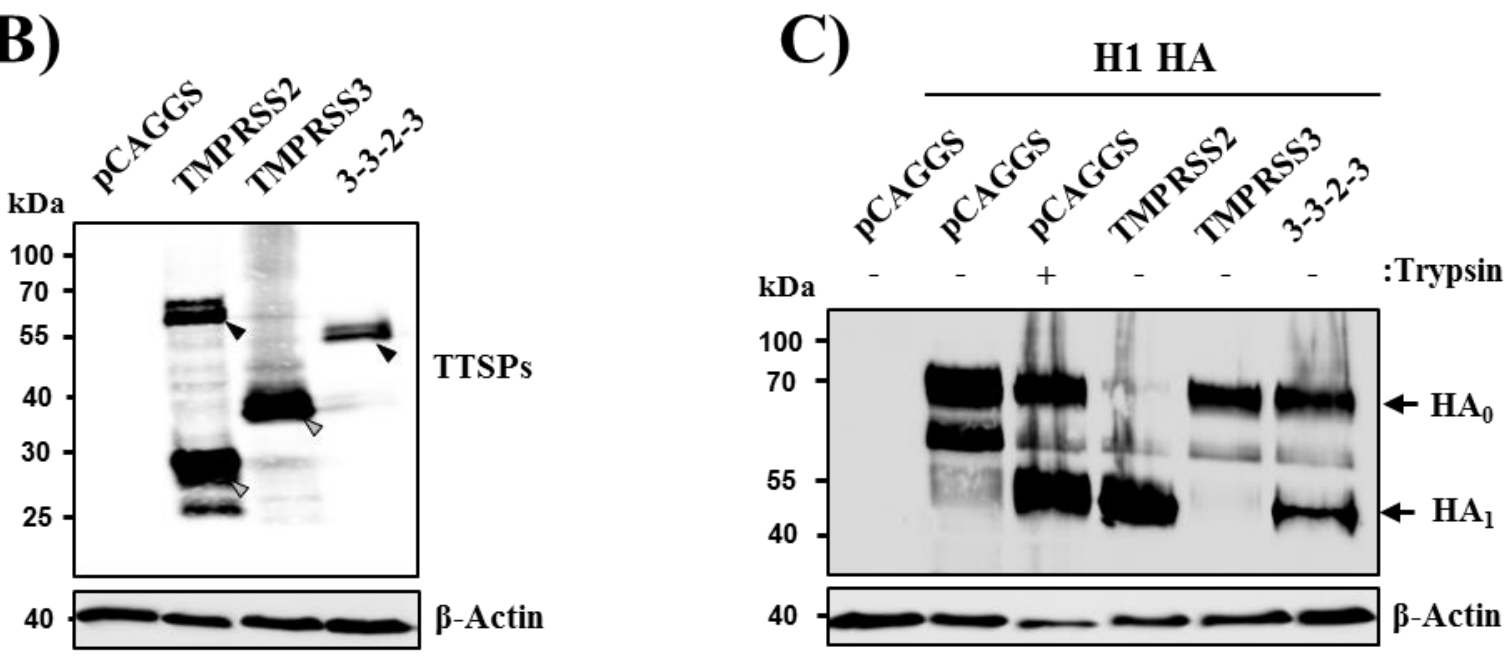

D)
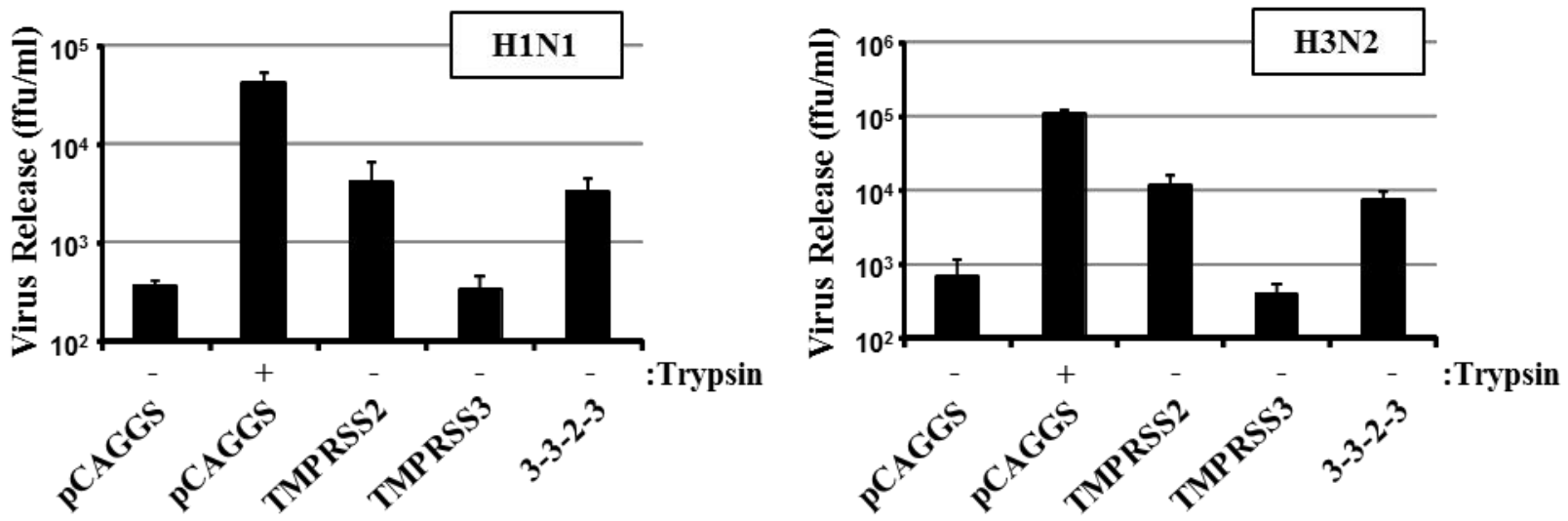
Figure 5

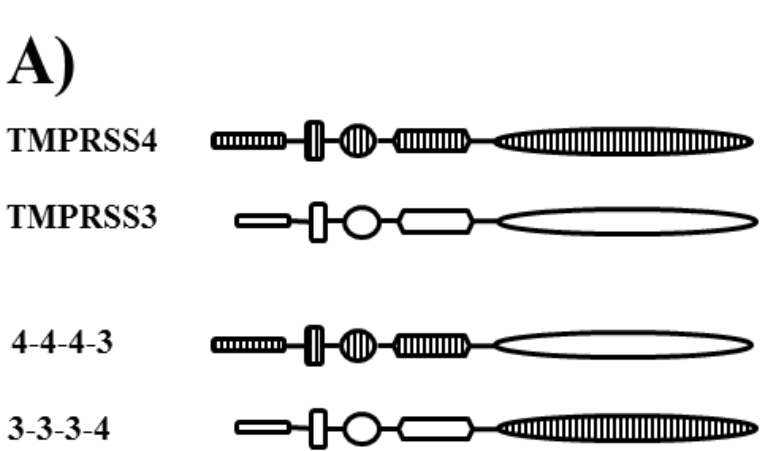

cytoplasmic $\square$ transmembrane

SRCR

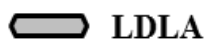

proteolytic

C)
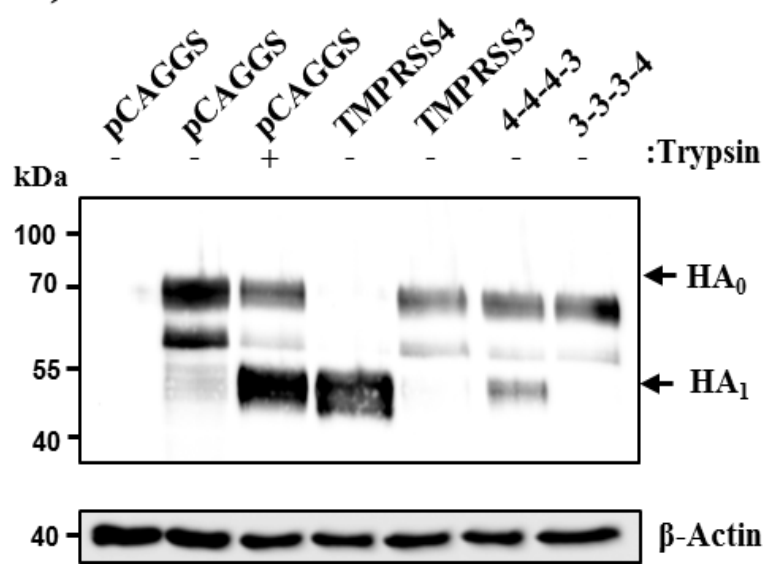

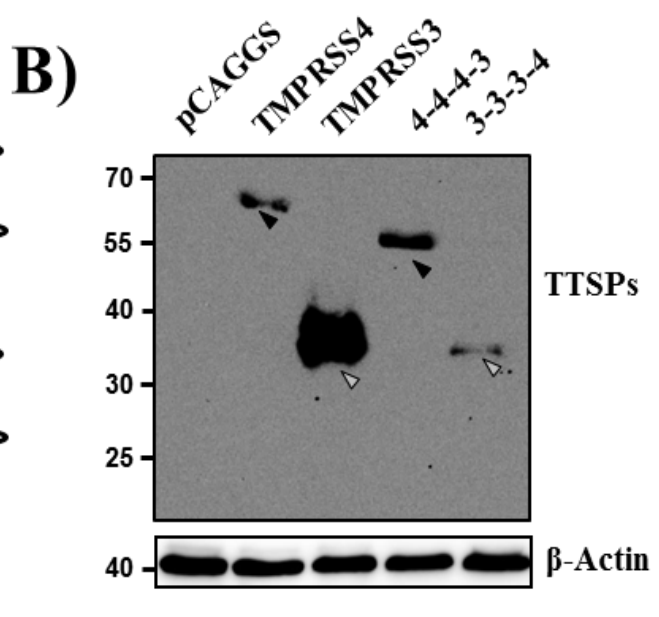

D)

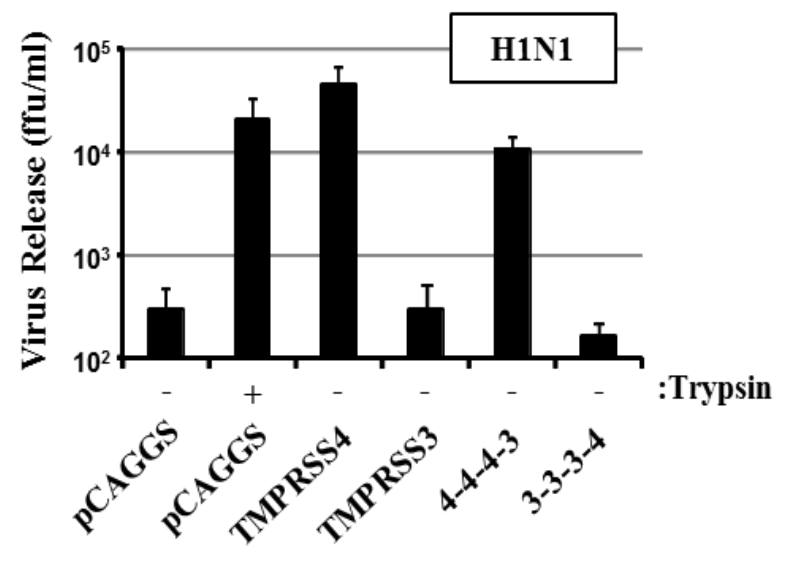

E)

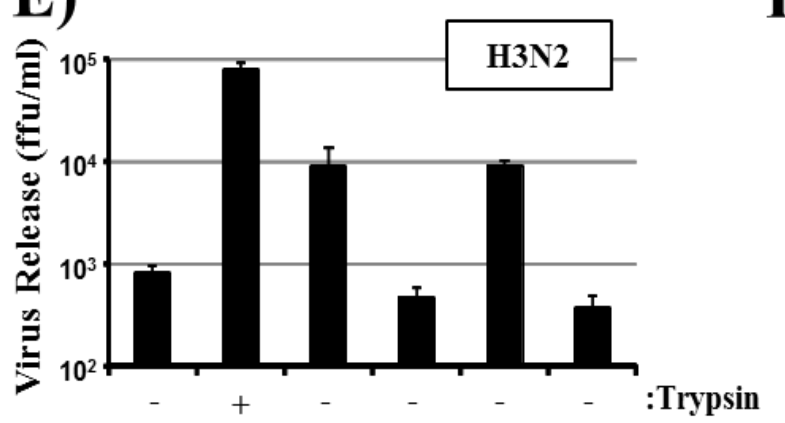

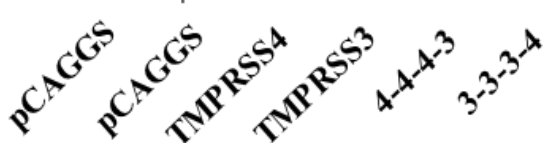

F)
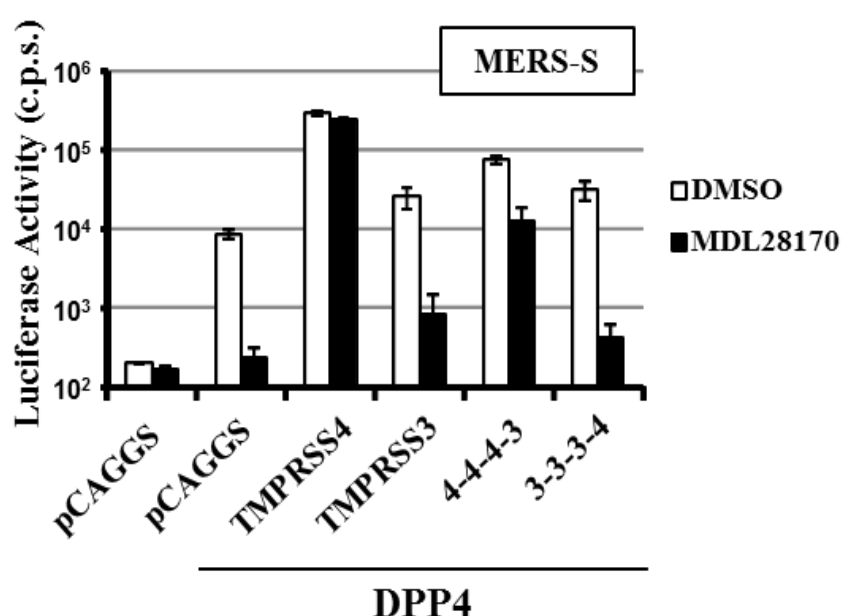

Page $\mid 75$ 
Figure 6

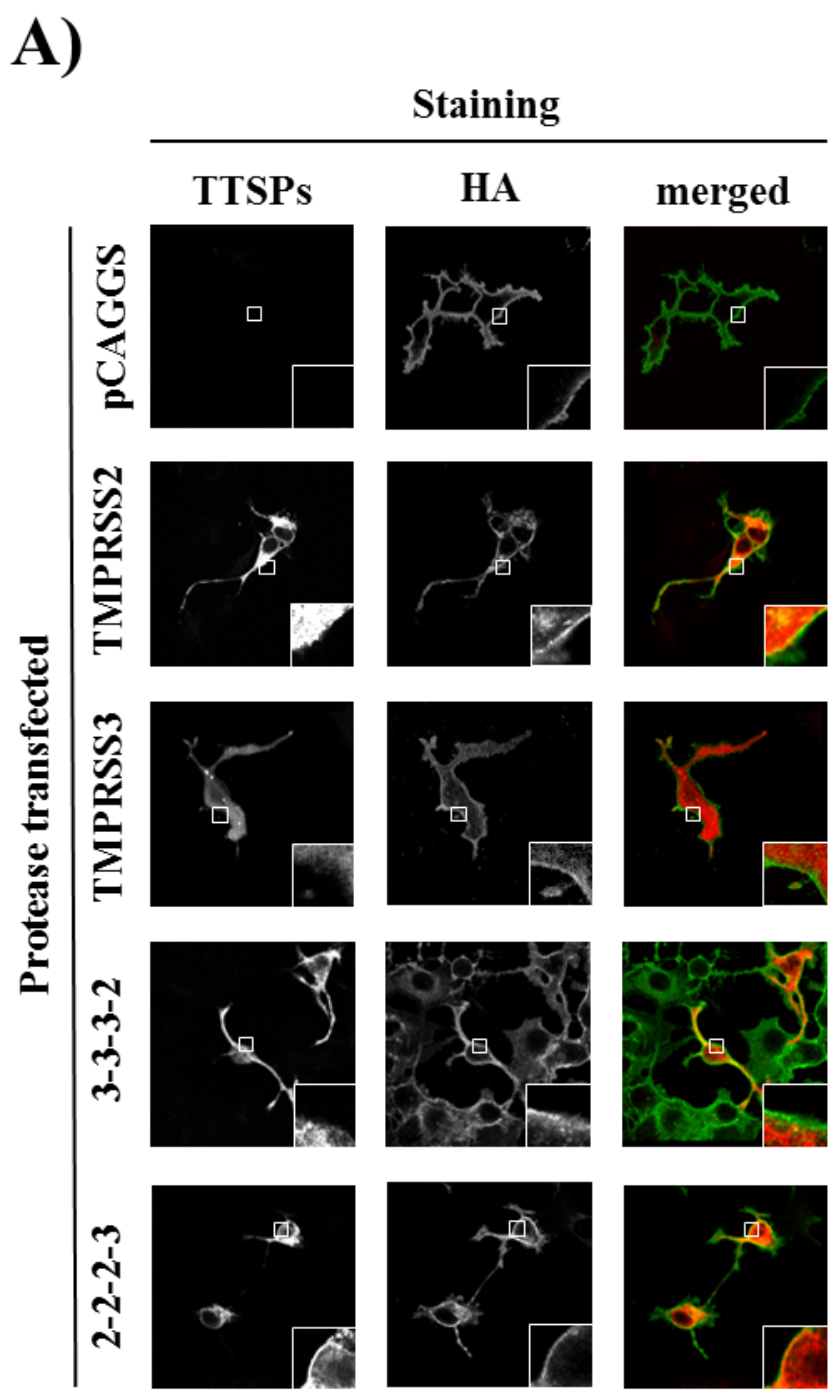

B)

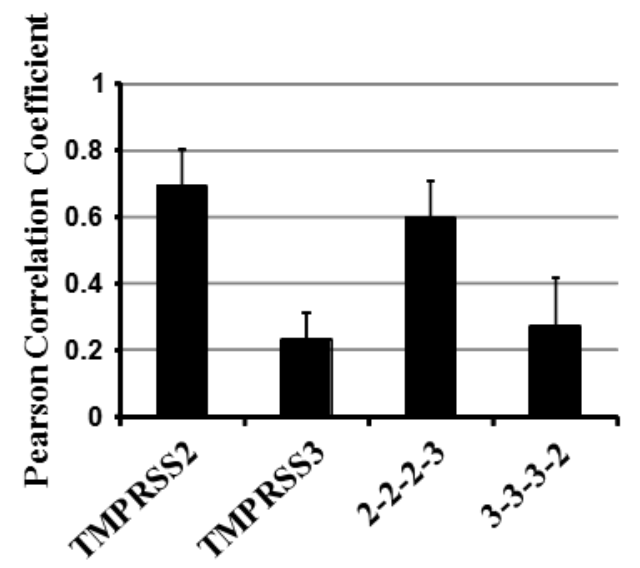


Figure 7
A)
B)
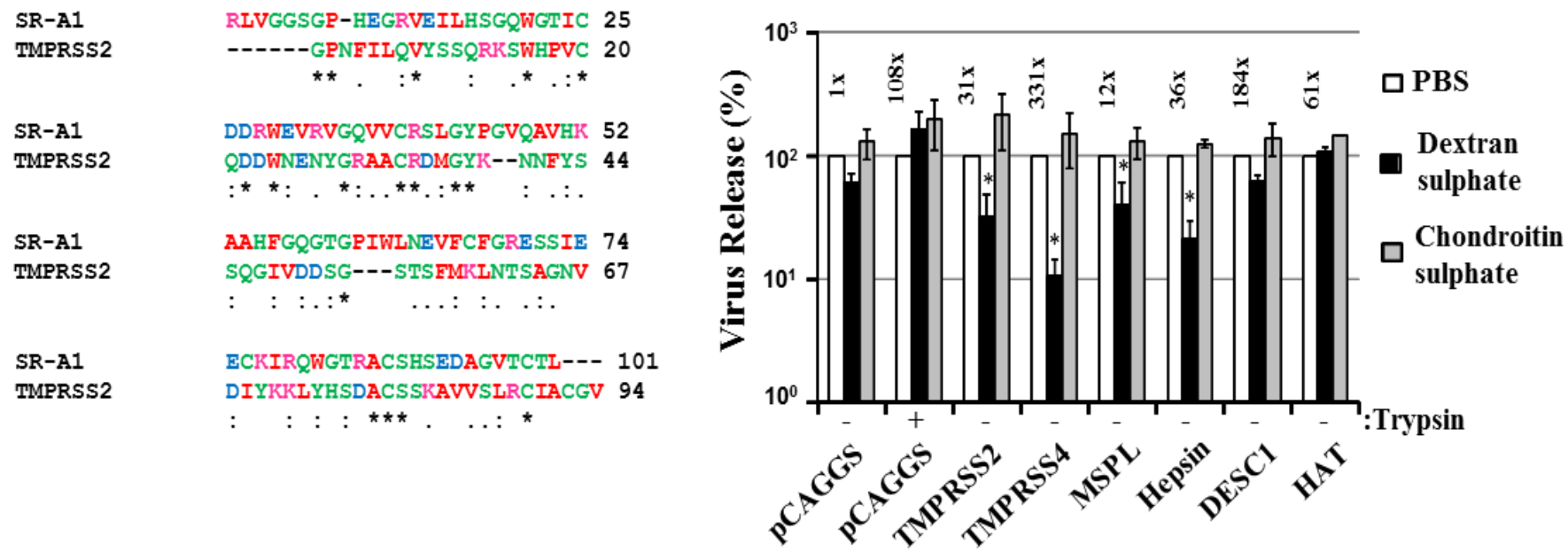

C)

Caco-2

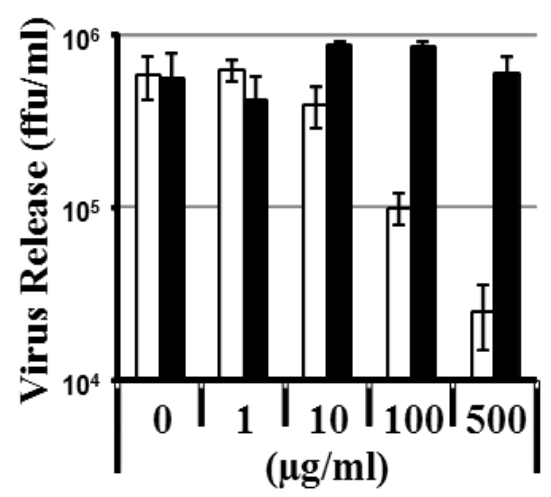

D)

pCAGGS TMPRSS4 DESC1

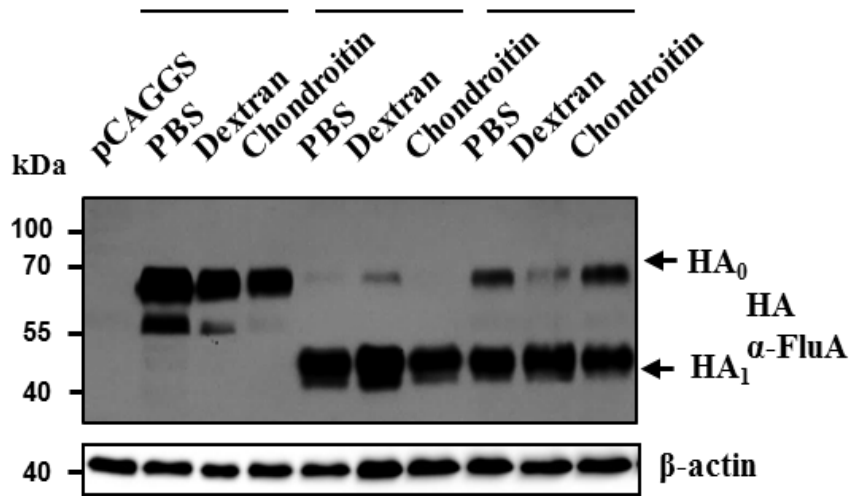

Calu-3

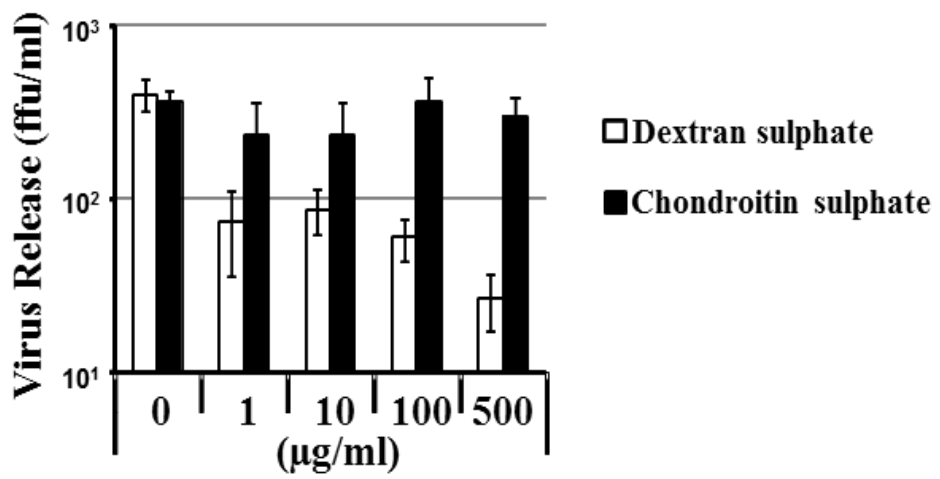

E)

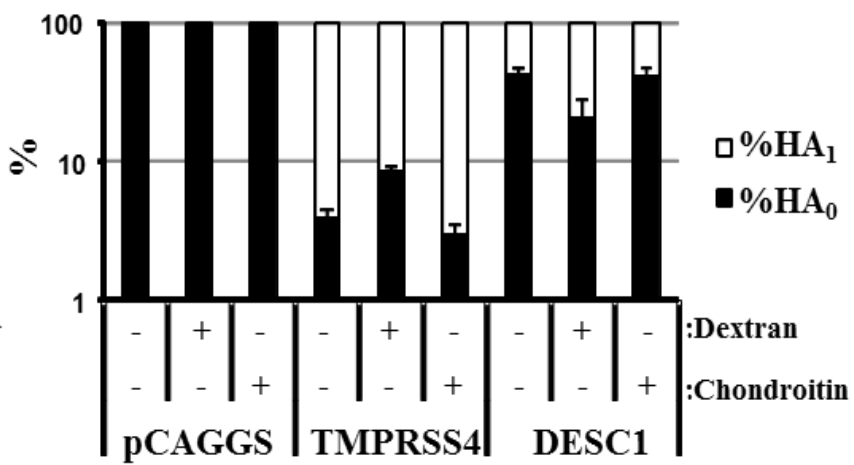




\title{
Third manuscript
}

\section{TMPRSS2 isoforms 1 activates respiratory viruses and is expressed in viral target cells}

\author{
Zmora. P., Moldenhauer, A.S., Pöhlmann, S. \\ PLOS ONE, 2015, 10: e0138380, doi: 10.1371/journal.pone.0138380
}

Individual contribution:

Design and conduct of the experiments. Bioinformatical analysis of TMPRSS2 isoforms (Fig. 1A). Construction of the plasmid encoding TMPRSS2 isoform 1 and determination of TMPRSS2 isoforms expression in transiently transfected 293T cells (Fig. 1B). Analysis of the TMPRSS2 isoforms expression in different organs/cell lines via RT-PCE (Fig. 2). Microscopy analysis of TMPRSS2 isoforms and hemagglutinin localization (Fig. 3A) and statistical analysis of Person correlation coefficient (Fig. 3B). Western blot analysis of emerging coronaviruses $\mathrm{S}$ protein cleavage by TMPRSS2 isoforms (Fig. 5A) and determination whether TMPRSS2 isoform 1 activates the S-driven virus-cell fusion (Fig. 5B). Writing parts of the manuscript (Material and Methods, Results) 


\title{
TMPRSS2 Isoform 1 Activates Respiratory Viruses and Is Expressed in Viral Target Cells
}

\author{
Pawel Zmora, Anna-Sophie Moldenhauer, Heike Hofmann-Winkler, Stefan Pöhlmann* \\ Infection Biology Unit, German Primate Center, Göttingen, Germany \\ * spoehlmann@dpz.eu
}

\begin{abstract}
The cellular protease TMPRSS2 cleaves and activates the influenza virus hemagglutinin (HA) and TMPRSS2 expression is essential for viral spread and pathogenesis in mice. Moreover, severe acute respiratory syndrome coronavirus (SARS-CoV) and other respiratory viruses are activated by TMPRSS2. However, previous studies on viral activation by TMPRSS2 focused on a 492 amino acids comprising form of the protein (isoform 2) while other TMPRSS2 isoforms, generated upon alternative splicing of the tmprss 2 mRNA, have not been characterized. Here, we show that the mRNA encoding a TMPRSS2 isoform with an extended $\mathrm{N}$-terminal cytoplasmic domain (isoform 1 ) is expressed in lung-derived cell lines and tissues. Moreover, we demonstrate that TMPRSS2 isoform 1 colocalizes with HA and cleaves and activates HA. Finally, we show that isoform 1 activates the SARS-CoV spike protein for cathepsin L-independent entry into target cells. Our results indicate that TMPRSS2 isoform 1 is expressed in viral target cells and might contribute to viral activation in the host.
\end{abstract} Winkler H, Pöhlmann S (2015) TMPRSS2 Isoform Activates Respiratory Viruses and Is Expressed in Viral Target Cells. PLoS ONE 10(9): e0138380. doi:10.1371/journal.pone. 0138380

Editor: Vincent Jacobus Munster, NIH, UNITED STATES

Received: June 11, 2015

Accepted: August 28, 2015

Published: September 17, 2015

Copyright: @ 2015 Zmora et al. This is an open access article distributed under the terms of the Creative Commons Attribution License, which permits unrestricted use, distribution, and reproduction in any medium, provided the original author and source are credited.

Data Availability Statement: All relevant data are presented in the paper.

Funding: This work was supported by DFG (PO 716/ 6-1) and the Göttingen Graduate School for Neurosciences, Biophysics, and Molecular Biosciences (DFG Grant GSC 226/1 and DFG Grant GSC 226/2).

Competing Interests: The authors have declared that no competing interests exist.

\section{Introduction}

Respiratory viruses pose a significant threat to human health. In particular, annual influenza epidemics are associated with several hundred thousand deaths every year, and interspersed pandemics may wreck even greater havoc [1] , as documented by the 1918 Spanish influenza, which caused 30 to 50 million deaths [2]. Antiviral drugs against influenza are available but their effectiveness is compromised by frequent acquisition of viral resistance. Moreover, no drugs with broad antiviral activity are available to combat emerging and highly virulent respiratory viruses, including severe acute respiratory syndrome coronavirus (SARS-CoV) and Middle East respiratory syndrome (MERS) CoV. In order to close this gap, novel antiviral strategies are being sought, which allow inhibition of a broad spectrum of viruses and which are associated with a high barrier against resistance development. Host cell factors which are essential for viral spread but dispensable for cellular survival are attractive targets for such approaches to antiviral therapy.

The surface proteins of influenza A viruses (FLUAV) and coronaviruses, termed hemagglutinin (HA) and spike (S), respectively, facilitate viral binding to host cells and fusion of the viral envelope with a host cell membrane [3]. These proteins are synthesized as inactive 
precursors and are converted by host cell proteases into their active forms [4] , a process referred to as activation in the remainder of the manuscript. Activation is essential for viral infectivity and the responsible enzymes are potential targets for antiviral therapy. Recent work indicated that several respiratory viruses hijack the type II transmembrane serine protease (TTSP) TMPRSS2 for their activation. Thus, TMPRSS2 was shown to cleave and activate the HA proteins of diverse FLUAV in culture [ $\underline{5}-\underline{8}]$ and studies with tmprss2-deficient mice indicated that TMPRSS2 expression is essential for spread and pathogenesis of FLUAV [9-11]. Moreover, augmented TMPRSS2 expression was found to be associated with increased risk of severe influenza upon infection with the $2009 \mathrm{H} 1 \mathrm{~N} 1$ pandemic virus and with increased susceptibility to H7N9 FLUAV infection [12]. Finally, TMPRSS2 was shown to activate diverse CoVs [13-16], parainfluenza virus [17], human metapneumovirus [18] and hepatitis $\mathrm{C}$ virus [19] in cell culture and might contribute to viral spread in the host. Remarkably, the absence of TMPRSS2 does not compromise development or homeostasis [20], indicating that TMPRSS2-specific inhibitors might exert broad antiviral activity without causing substantial unwanted side effects.

Alternative splicing of the messenger RNAs produced from several TTSP genes has been reported $[21,22]$, and may result in the production of isoforms with different functional properties. For instance, the presence of an alternative first exon in the corin mRNA can alter surface localization of the protein and conversion of the zymogen form into the mature enzyme [21]. It has been suggested that the tmprss 2 transcript can be alternatively spliced $[\underline{23}, 24]$, which may result in the production of two isoforms which differ only in the N-terminal, cytoplasmic tail: Isoform 1 contains 37 amino acids in its tail which are not present in isoform 2 . However, previous analyses of TMPRSS2 in the context of viral infections have exclusively focused on isoform 2 while expression of isoform land its ability to cleave and activate the surface proteins of respiratory viruses has not been assessed.

Here, we show that mRNA encoding isoform 1 is expressed in certain lung-derived cell lines and tissues and that the protein can activate FLUAV and the S proteins of SARS-CoV and MERS-CoV (SARS-S, MERS-S), suggesting that isoform 1 could promote viral spread in the infected host.

\section{Materials and Methods}

\section{Bioinformatic analysis}

Nucleotide and amino acid sequences were analyzed using the BLAST server at NCBI. The alignment of the amino acid sequences of the $\mathrm{N}$-termini of TMPRSS2 isoform 1 (NP_001128571.1) and isoform 2 (NP_005647.3) was constructed using the Clustal Omega software.

\section{Plasmid construction}

Expression plasmids for H1 and H3 FLUAV HA, SARS-S, MERS-S, ACE2 and DESC1 have been described previously [25]. For construction of the plasmids containing TMPRSS3 and TMPRSS2 isoform 2, the indicated genes were PCR-amplified from previously described plasmids and cloned into pCAGGS via the EcoRI and XhoI restriction sites. To PCR-amplify TMPRSS2 transcript variant 1, an oligonucleotide (Sigma Aldrich) encoding the extended Nterminal sequences, 5'-ATGCCCCCTGCCCCGCCCGGAGGTGAAAGCGGGTGTGAGGA GCGCGGCGCGGCAGGTCATATTGAACATTCCAGATACCTATCATTACTCGATGCT GTTGATAAAGCAAG-3' was employed as 5' primer and TMPRSS2 transcript variant 2 was used as template. Subsequently, the PCR-amplificate was cloned into pCAGGS via the EcoRI and XhoI restriction sites. Expression plasmids for proteases with an $\mathrm{N}$-terminal myc-tag 
were generated by PCR using the above-described plasmids as templates, as reported previously [25]. The identity of all PCR-amplified sequences was verified by automated sequence analysis.

\section{Cells and viruses}

The cell lines 293T (ATCC CRL-3216), Calu-3 (ATCC HTB-55), Caco-2 (ATCC HTB-37), EA-hy (ATCC CRL-2922), BEAS-2b (ATCC CRL-9609), NCI-H292 (ATCC CRL-184), NCI-H727 (ATCC CRL-5815), A549 (ATCC CCL-185) and COS-7 (ATCC CRL-1651) were grown in Dulbecco's modified Eagle's medium (DMEM, PAN Biotech), while LNCaP (ATCC CRL-1740) and NCI-H358 cells (ATCC CRL-5807) were propagated in RPMI-1640 medium (PAN Biotech), and MDCK cells (ATCC CRL-2936) were grown in minimum essential medium (Gibco). All media were supplemented with 10\% fetal bovine serum (Biochrome), 100 $\mathrm{U} / \mathrm{ml}$ penicillin and streptomycin (PAN Biotech). The cells were cultured in humidified atmosphere containing $5 \% \mathrm{CO}_{2}$. All cell lines were obtained from collaborators and were regularly checked for mycoplasma contamination. The FLUAV A/PR/8/34 (H1N1) and A/Panama/ 2007/99 (H3N2) were propagated in the chorioallantoic cavities of 10-days-old embryonated hen eggs (Valo BioMedia, Germany) for $48 \mathrm{~h}$ at $37^{\circ} \mathrm{C}$. Thereafter, the eggs were euthanized by an overnight incubation at $4^{\circ} \mathrm{C}$ and the allantoic fluid was harvested. Before propagation in eggs, the FLUAV A/Panama/2007/99 was recovered from a reverse genetics system [26]. The amount of infectious units within the chorioallantoic fluid was determined by focus formation assay, as described previously [25]. In brief, serial 10-fold dilutions of samples were prepared and added onto MDCK cells. After $1 \mathrm{~h}$ of incubation, the medium was replaced by infection medium containing Avicel overlay and $2.5 \mu \mathrm{g} / \mathrm{ml} \mathrm{N}$-acetylated trypsin (Sigma). After an incubation period of $24 \mathrm{~h}$, the cells were fixed with $4 \%$ formalin in PBS and incubated for $1 \mathrm{~h}$ with anti-FLUAV polyclonal antibody from goat (Millipore). Subsequently, the cells were washed and incubated for $1 \mathrm{~h}$ with anti-goat-HRP antibodies (KPL), washed again and incubated for 10 min with True Blue substrate (KPL). Finally, foci were counted and viral titers were calculated as focus forming units (FFU) per ml of culture supernatant.

\section{Analysis of mRNA expression in tissues and cells}

Total RNA was isolated from human cell lines with RNeasy Mini Kit (Qiagen), as recommended by the manufacturer. The Human MTC ${ }^{\mathrm{m}}$ Panel I (Clonetech) cDNA was used to analyze mRNA expression in human organs. This cDNA was obtained from pooled tissue samples from 1-15 Caucasian donors aged 18-69 and representing both sexes. Using a Cloned AMV First-Strand cDNA synthesis kit (Invitrogen) and random hexamers, the first strand cDNA synthesis was performed from $1 \mu \mathrm{g}$ of total RNA, previously treated with DNaseI (Roche), according to the manufacturer's protocol. The subsequent PCR was performed with Taq polymerase (New England Biolabs) using gene-specific primers for tmprss 2 transcript variant 1 (forward 5' GTG AAA GCG GGT GTG AGG A 3' and reverse 5'CTG TGC GGG ATA GGG GTT TT 3'), tmprss2 transcript variant 2 (forward 5'GCG AGG GGC GGG GAG CGC C 3' and reverse 5' GGT AGT ACT GAG CCG GAT GC 3') and GAPDH (forward 5’ ATG GGG AAG GTG AAG GTC GG 3' and reverse 5’ ATA CTT CTC ATG GTT CAC AC 3'). All PCRs were run for $40 \mathrm{cycles}$ of $30 \mathrm{sec}$ denaturation at $95^{\circ} \mathrm{C}, 30 \mathrm{sec}$ annealing at $58^{\circ} \mathrm{C}$, and $30 \mathrm{sec}$ elongation at $72^{\circ} \mathrm{C}$. Amplicons were analyzed by agarose gel electrophoresis.

\section{Western blot analysis}

For analysis of the expression of the TMPRSS2 isoforms, 293T cells were seeded into 6-well plates at a density of $2.8 \times 10^{5}$ cells/well, cultivated for $24 \mathrm{~h}$ and then transfected with plasmids 
encoding the proteases equipped with an $\mathrm{N}$-terminal myc tag or transfected with empty plasmid as control. After overnight incubation, the medium was replaced with fresh DMEM, and at $48 \mathrm{~h}$ post transfection the cells were washed with phosphate-buffered saline (PBS), resuspended in $100 \mu \mathrm{l}$ of $2 \mathrm{x}$ sodium dodecyl sulphate (SDS) loading buffer per well and then heated at $95^{\circ} \mathrm{C}$ for $30 \mathrm{~min}$. Protein samples were separated by SDS-PAGE and blotted onto a nitrocellulose membrane (Hartenstein). TMPRSS2 isoforms were detected using a mouse anti-myc antibody (Biomol) as the primary antibody and a horseradish peroxidase (HRP)-coupled antibody (Dianova) as the secondary antibody. Expression of $\beta$-actin, detected with anti- $\beta$-actin antibody (Sigma Aldrich), served as a loading control. Bound antibodies were detected using ECL Prime Western blotting detection kit (Amersham), according to the manufacturer's instructions. Image acquisition was performed with a ChemoCam Imager (Intas). For analysis of TMPRSS2 isoform expression by flow cytometry, 293T cells were transfected with plasmids encoding TMPRSS2 isoforms, as described above. At $48 \mathrm{~h}$ post transfection, the cells were detached, incubated with ice-cold ethanol for $10 \mathrm{~min}$ and stained with anti-TMPRSS2 antibody (Santa Cruz Biotechnology) diluted in 1\% saponin for $30 \mathrm{~min}$. The mouse IgG1 (R\&D Systems) served as isotype-matched control. Thereafter, the cells were washed three times with PBS and incubated with an AlexaFluor647-coupled anti-mouse antibody (Dianova) diluted in 1\% saponin. After 30 min of incubation with secondary antibody, the cells were washed two times with PBS and then fixed with $2 \%$ paraformaldehyde. The staining was analyzed with an LSRII flow cytometer (BD Biosciences) and FCS Express 4 Flow Research Edition software (DeNovo Software).

\section{Analysis of SARS-S and FLUAV HA cleavage by TMPRSS2 isoforms}

To determine cleavage of viral glycoproteins mediated by TMPRSS2 isoforms, 293T cells were seeded in 6-well plates at $2.8 \times 10^{5}$ cells/well, cultured for $24 \mathrm{~h}$ and then cotransfected with $6 \mu \mathrm{g}$ of plasmid encoding FLUAV HA of the $\mathrm{H} 1$ or $\mathrm{H} 3$ subtype or SARS-S with a C-terminal V5 tag and $0.1 \mu \mathrm{g}$ of plasmid encoding the indicated proteases, employing the calcium phosphate transfection protocol. At $48 \mathrm{~h}$ post transfection, the cells were harvested in PBS and treated with PBS or $250 \mu \mathrm{g} / \mathrm{ml}$ tosylsulfonyl phenylalanyl chloromethyl ketone (TPCK) trypsin (Sigma Aldrich) for $10 \mathrm{~min}$ at $37^{\circ} \mathrm{C}$ and processed for Western blot analysis as described above. The SARS-S protein with a C-terminal V 5 tag was detected by staining with mouse monoclonal antibody reactive against the V5 tag (Invitrogen), followed by incubation with an HRP-coupled anti-mouse secondary antibody (Dianova). The FLUAV HA cleavage was detected by staining with a goat anti-FLUAV polyclonal antibody (Millipore) for $\mathrm{H} 1$ subtype or with a rabbit antiH3 HA serum (Immune Technology) and HRP-coupled anti-goat or anti-rabbit antibodies (Dianova), respectively. As a loading control, the expression of $\beta$-actin was detected with anti$\beta$-actin antibodies (Sigma Aldrich).

\section{Activation of SARS-S- and MERS-S-mediated virus-cell fusion by TMPRSS2 isoforms}

For the analysis of the activation of SARS-S and MERS-S for virus-cell fusion, we employed a previously described retroviral pseudotyping system $[25,27]$. Briefly, 293T cells were seeded in T25 cell culture flasks at a density of $7.0 \times 10^{5}$ cells/flask, cultured for $24 \mathrm{~h}$ and then transfected by calcium phosphate precipitation with plasmids encoding for MLV gag-pol ( $3 \mu \mathrm{g})$, a fireflyluciferase harboring MLV vector $(6 \mu \mathrm{g})$, and a plasmid encoding for SARS-S (3 $\mu \mathrm{g})$. The transfection medium was replaced by fresh DMEM after overnight incubation, and vector-containing supernatants were harvested at $48 \mathrm{~h}$ post transfection by filtration through $0.45 \mu \mathrm{m}$ filters, aliquoted and stored at $-80^{\circ} \mathrm{C}$. For analysis of SARS-S-/MERS-S-mediated entry, $293 \mathrm{~T}$ cells 
were transiently cotransfected with plasmids encoding ACE2 or DPP4 $(2 \mu \mathrm{g})$, respectively, and the indicated proteases $(0.3 \mu \mathrm{g})$ or an empty plasmid as a control. The transfection medium was changed after overnight incubation, the cells were resuspended in fresh DMEM and seeded into 96-well plates. At $48 \mathrm{~h}$ post transfection, the cells were preincubated with dimethyl sulfoxide (DMSO) or $10 \mu \mathrm{M}$ cathepsin B and L inhibitor MDL 28170 (Calbiochem) for $1 \mathrm{~h}$ and then incubated with $50 \mu \mathrm{l}$ of infectivity-normalized pseudotypes for $8 \mathrm{~h}$. Thereafter, $50 \mu \mathrm{l}$ of fresh medium was added and the luciferase activities in cell lysate were determined at $72 \mathrm{~h}$ post transduction using a commercially available kit (Beetle Juice (PJK)).

\section{Proteolytic activation of influenza A viruses mediated by TMPRSS2 isoforms}

To determine whether the TMPRSS2 isoforms can activate FLUAV, 293T cells were seeded in 6-well plates at a density of $2.8 \times 10^{5}$ cells/well and cultured for $24 \mathrm{~h}$. Then, the cells were transiently transfected with $6 \mu \mathrm{g}$ of plasmids encoding the proteases or empty plasmid, which served as a control, using the calcium phosphate transfection method. After overnight incubation, the medium was replaced by fresh DMEM. At $24 \mathrm{~h}$ post transfection, the cells were gently washed with PBS and then incubated with DPBS with $\mathrm{Mg}^{2+}$ and $\mathrm{Ca}^{2+}$ (PAN Biotech) supplemented with $0.2 \%$ bovine serum albumine (BSA) (MACS Miltenyi Biotec) containing FLUAV A/PR/8/34 or A/Panama/2007/99 at a multiplicity of infection (MOI) of 0.01 or 0.1 , respectively. After $1 \mathrm{~h}$ of incubation at $37^{\circ} \mathrm{C}$ in a humidified atmosphere, the cells were gently washed with PBS, and fresh infection medium (DMEM supplemented with $0.2 \%$ BSA, penicillin and streptomycin) was added. To analyze virus release, the culture supernatants were collected at $48 \mathrm{~h}$ post infection. The amount of infectious units within the culture supernatants was determined by focus formation assay, as described above.

\section{Cellular localization of TMPRSS2 isoforms}

To analyze the cellular localization of TMPRSS2 isoforms, COS-7 cells were transiently transfected with $6 \mu \mathrm{g}$ of plasmids encoding TMPRSS2 isoforms or empty plasmid as control. After overnight incubation, the transfection medium was replaced with fresh DMEM. At $24 \mathrm{~h}$ post transfection, the cells were washed with PBS, and incubated for $1 \mathrm{~h}$ with DPBS with $\mathrm{Ca}^{2+}$ and $\mathrm{Mg}^{2+}$, supplemented with $0.2 \% \mathrm{BSA}$, containing FLUAV at an MOI of 0.5 . Thereafter, the cells were washed with PBS and fresh infection medium was added. At $24 \mathrm{~h}$ post infection, the cells were fixed with ice cold methanol, blocked with $3 \%$ BSA for $1 \mathrm{~h}$, and then stained with mouse anti-TMPRSS2 and rabbit-anti-PR8 HA antibodies (Santa Cruz and Sino Biological, respectively). After $1 \mathrm{~h}$ of incubation with primary antibodies, the cells were washed three times with PBS, and incubated for $1 \mathrm{~h}$ with anti-mouse and anti-rabbit secondary antibodies, coupled to Rhodamine Red-X and FITC (Dianova), respectively. After three final washing steps, the cells were stained with Vectashield mounting medium (Vector Laboratories) and analyzed with a Zeiss LSM 5 laser scanning microscope. Image capture was performed with Pascal Software (Zeiss) and Fiji software was used for image analysis and calculation of Pearson Correlation Coefficient.

\section{Results}

\section{Expression and autocatalytic activation of TMPRSS2 isoforms in transfected cells}

Alternative splicing of the tmprss 2 transcript is believed to generate mRNAs encoding for at least two different isoforms of the protein, isoforms 1 and $2[\underline{23}, \underline{24}]$ : Both isoforms contain 
identical transmembrane and extracellular domains but isoform 1 exhibits an extended $\mathrm{N}$-terminal cytoplasmic domain, harboring a 37 amino acids comprising sequence which is not present in isoform 2 (Fig 1A). Isoform 2 has been shown to activate the envelope proteins of several viruses while it is at present unknown whether isoform 1 can also act as a viral activator.

A)

Isoform 1 MPPAPPGGESGCEERGAAGH 20

Isoform 2 - - - - - - -

IsOform 1 IEHSRYLSLLDAVDNSKMAL 40

Isoform 2 - - - - 3

Isoform 1 NSGSPPAIGPYYENHGYQPE 60

IsOform 2 NSGSPPAIGPYYENHGYQPE 23
B)

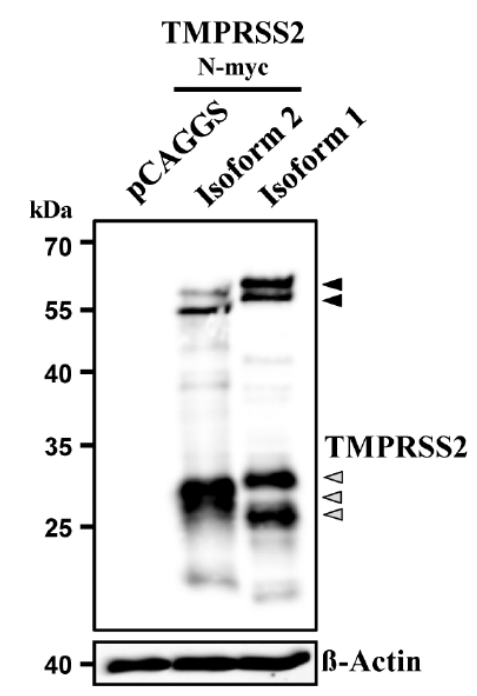

C)

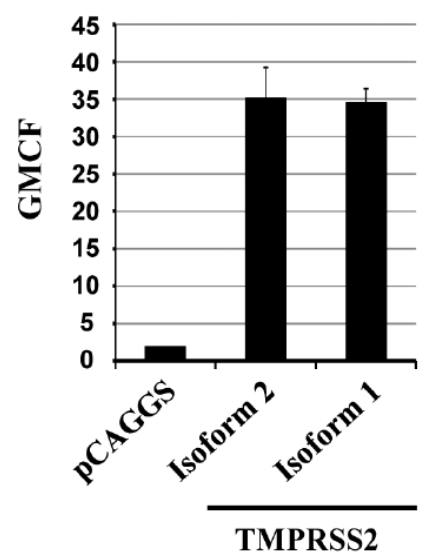

Fig 1. Expression of TMPRSS2 isoforms 1 and 2. (A) Sequence alignment of the N-termini of TMPRSS2 isoforms 1 and 2. Identical amino acids are marked with stars. Amino acids absent in isoform 2 are marked with '- $-^{-}$. (B) Plasmids encoding TMPRSS2 isoform 1 and isoform 2, both equipped with an $\mathrm{N}$ terminal myc tag, were transiently transfected into 293T cells. Empty plasmid (pCAGGS) served as a negative control. Protease expression in cell lysates was detected via Western blotting with anti-myc antibody. The $\beta$-actin expression served as a loading control. Black-filled arrowheads indicate the zymogen forms, while grey-filled arrowheads highlight cleavage products resulting from protease activation. (C) 293T cells were transfected as described for panel B but protease expression was determined using flow cytometry with an anti-TMPRSS2 antibody. The geometric mean channel fluorescence (GMCF)

measured in a representative experiment performed with triplicate samples is shown. Error bars indicate standard deviations. Similar results were obtained in two independent experiments.

doi:10.1371/joumal.pone.0138380.g001 
Moreover, it is unknown whether the isoforms are differentially expressed in viral target cells. To address these questions, we cloned the transcript variants encoding for each individual isoform and analyzed whether the proteins are efficiently expressed in transfected cells. The expression levels of TMPRSS2 isoforms 1 and 2 were comparable as determined by Western blot (Fig 1B) and flow cytometry (Fig 1C) and unprocessed isoform 1 exhibited a slightly higher molecular weight than its counterpart (Fig 1B), in keeping with expectations (expected molecular weight isoform $1,57.7 \mathrm{kDa}$; expected molecular weight isoform 2, $53.9 \mathrm{kDa}$ ). Both proteins were autocatalytically activated (i.e. the inactive zymogen form was cleaved between the $\mathrm{C}$-terminal protease domain and the remainder of the protein, which allows the protease domain to undergo conformational changes and to transit into an active state), as evidenced by the presence of $\mathrm{N}$-terminal cleavage products. A single $\mathrm{N}$-terminal fragment was observed for isoform 2 while two fragments were detected for isoform 1 (Fig 1B), suggesting that the presence of an extended $\mathrm{N}$-terminus in isoform 1 does not alter the efficiency of TMPRSS2 autoactivation but might change cleavage specificity.

\section{Transcript variant 1 is expressed in influenza A virus target cell lines and organs}

We next determined whether transcript variant 1 (which encodes isoform 1 ) is expressed in tissues and cell lines. For this, we employed RT-PCR with primer sets specific for tmprss 2 transcript variant 1 and 2, respectively. Primers specific for variant 1 amplified their target sequence from a plasmid encoding isoform 1 but not isoform 2, confirming that the PCR was specific. Similarly, the PCR designed to amplify transcript variant 2 was negative when a plasmid encoding isoform 1 was used as template but generated the expected amplificate from cDNA prepared from several cell lines and tissues, indicating that also this PCR was specific. In this context, it should be noted that the forward primer used for detection of transcript variant 2 binds to a 5 '-untranslated region which is absent from the plasmid encoding isoform 2. Therefore, the respective PCR was negative.

The investigation of tissue samples revealed robust expression of transcript variant 1 in lung, kidney, liver and pancreas, which were reported to express tmprss 2 mRNA (isoforms were not discriminated in previous studies) $[\underline{23}, \underline{28}]$ (Fig 2, left panel). In contrast, mRNA levels in muscle and placenta were reduced and no expression of transcript variant 1 was detected in heart and brain. A similar result was obtained when expression in cell lines was determined (Fig 2, right panel): Transcript variant 1 was expressed in several but not all cell lines tested,
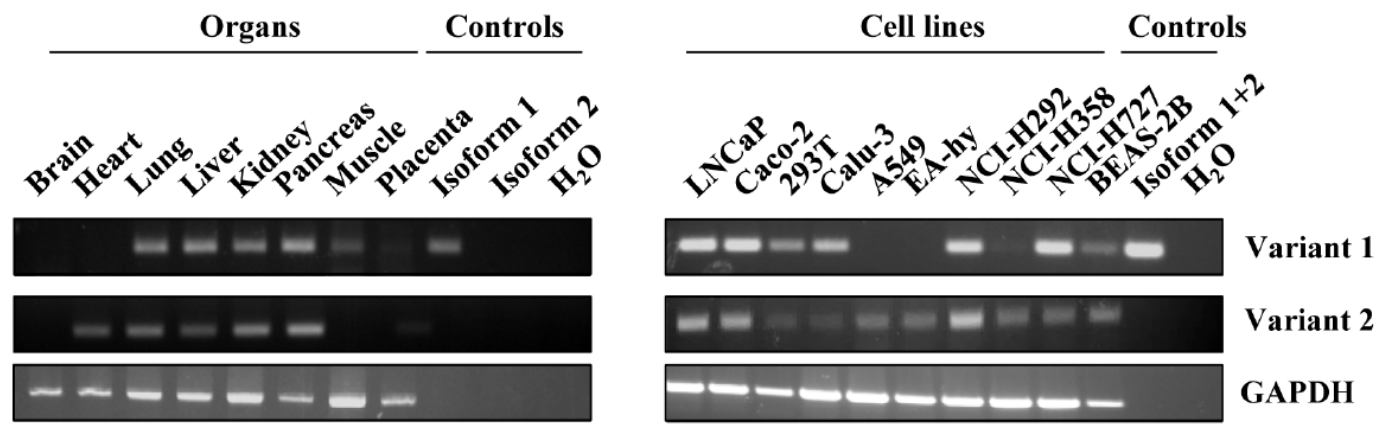

Fig 2. Expression of tmprss2 transcript variants in human organs and cell lines. RT-PCR analysis of the expression of tmprss 2 transcript variants 1 and 2 in organ samples from adult men (left panel) or from cell lines of human origin (right panel). Expression of GAPDH was assessed in parallel. Plasmids encoding isoform 1 and 2 (left panel) or a mix of both plasmids (right panel) served as positive control. Similar results were obtained in two separate experiments.

doi:10.1371/joumal.pone.0138380.g002 
including the lung-derived cell line Calu-3 and the colon-derived cell line Caco-2, which were previously shown to produce TMPRSS 2 protein $[\underline{5}, \underline{6}]$. The expression pattern of transcript variant 2 was similar but not identical to that of variant 1 . Thus, all organs and cell lines positive for transcript variant 1 also expressed variant 2 except for muscle tissue, which was negative for variant 2. Conversely, heart tissue and the cell lines A549 and EA-hy were negative for transcript variant 1 but expressed variant 2. Collectively, tmprss 2 transcript variant 1 and variant 2 exhibit an overlapping but not identical expression pattern. Moreover, our results indicate that both isoforms are expressed in lung tissue and several lung-derived cell lines (including Calu-3 and $\mathrm{NCIH} 292$ ) and are thus present in FLUAV targets.

\section{TMPRSS2 isoform 1 and 2 colocalize with the influenza A virus hemagglutinin}

Activation of FLUAV by TMPRSS2 in infected cells requires that HA comes into contact with the protease. In order to address whether HA and TMPRSS2 isoform 1 colocalize, immunofluorescence staining and Fiji analysis of COS-7 cells transiently expressing TMPRSS2 isoforms and infected with FLUAV was employed. We observed colocalization of HA with both TMPRSS2 isoforms in infected cells (Fig $3 \mathrm{~A}$ and $3 \mathrm{~B}$ ). However, we also noted differences in the cellular localization of isoform 1 and 2: although both isoforms were found at or near the cell surface, where they colocalized with FLUAV HA, more cytoplasmic puncta were observed in

A)

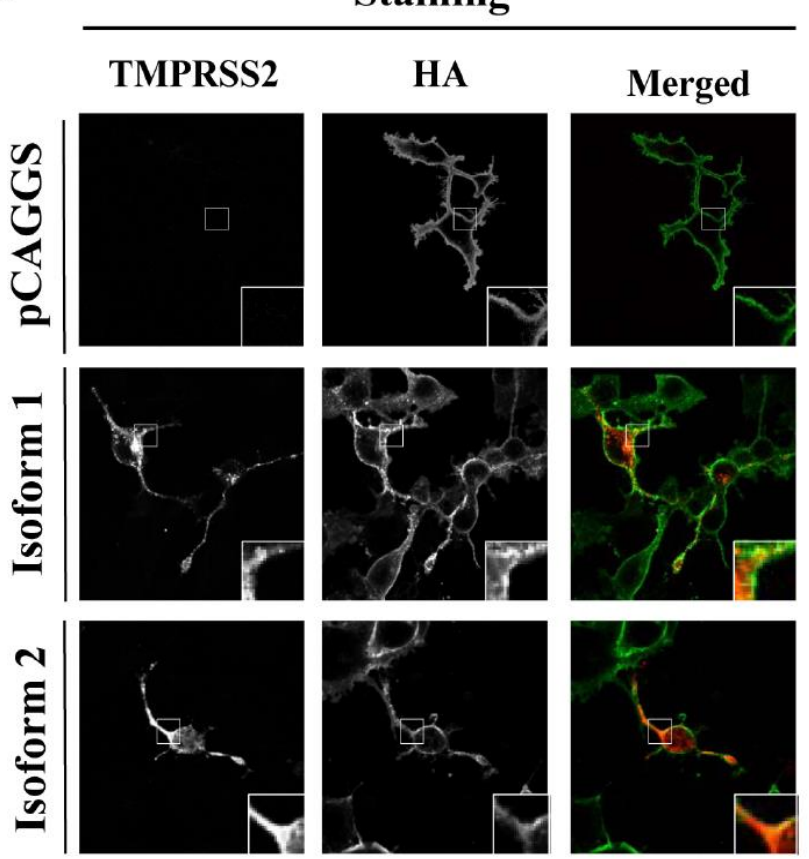

B)

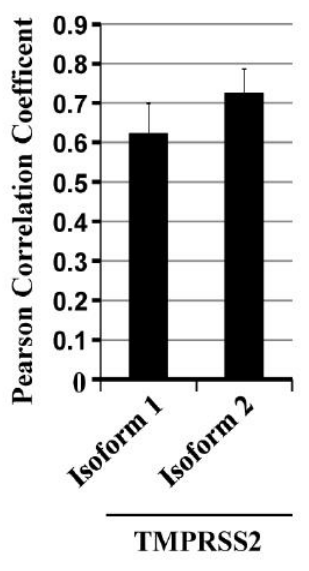

Fig 3. TMPRSS2 isoforms 1 and 2 colocalize with hemagglutinin. (A) COS-7 cells were transfected with plasmids encoding TMPRSS2 isoform 1 or isoform 2 or with empty plasmid which served as negative control. Subsequently, the cells were infected with FLUAV A/PR/8/34 (H1N1) at an MOI 0.5. At 24 $\mathrm{h}$ post infection, the cells were stained for FLUAV-HA (green) and TMPRSS2 isoforms (red) and images were taken at $63 \times$ magnification. White squares show examples of colocalization of HA and TMPRSS2 (yellow signals) and were digitally magnified $2.5 \mathrm{x}$ from the original images. Similar results were obtained in three separate experiments. (B) Images obtained in (A) were analyzed with Fiji software, which allows calculation of the Pearson Correlation Coefficient (PCC), a measure for colocalization. The average PCC measured in three separate experiments is shown. For each experiment, $6-8$ cells were analyzed. Error bars indicate standard error of the mean (SEM).

doi:10.1371/joumal.pone.0138380.g003 
cells expressing isoform 1 as compared to isoform 2, which was more homogenously distributed within the cells (Fig $3 \mathrm{~A})$. Thus, the presence of an extended N-terminus in isoform 1 does not interfere with HA colocalization but might modestly alter the intracellular localization of TMPRSS2.

\section{TMPRSS2 isoform 1 cleaves and activates the glycoproteins of influenza A viruses}

In order to determine whether TMPRSS2 isoform 1 can activate FLUAV, we first assessed cleavage of FLUAV HA. For this purpose, we coexpressed the HA proteins of FLUAV A/South Carolina/1/1918 (H1N1) or FLUAV A/Hong Kong/1/1968 (H3N2) and proteases. Cleavage of the $\mathrm{HA}$ precursor, $\mathrm{HA}_{0}$, was observed upon treatment of cells with trypsin and upon coexpression of DESC1 and TMPRSS2 isoform 2, in keeping with previous reports $[\underline{5}, \underline{25}]$ (Fig 4A). Additionally, HA proteolysis was detected upon coexpression of TMPRSS2 isoform 1. In contrast, the proteolytic cleavage products were not observed upon treatment of cells with PBS or upon coexpression of TMPRSS3 (Fig 4A), as expected [25].

We next asked whether cleavage results in HA activation. To this end, we determined the spread of FLUAV A/PR/8/34 (H1N1) and FLUAV A/Panama/2007/99 (H3N2) in 293T cells transfected to express the indicated proteases. Treatment of cells with PBS or expression of TMPRSS3 did not promote viral spread ( $\underline{\text { Fig } 4 \mathrm{~B}}$ ), as expected $[\underline{5}, \underline{25}]$. In contrast, expression of TMPRSS2 isoform 2 and DESC1 or treatment of the cells with trypsin boosted viral spread (Fig $\underline{4 \mathrm{~B}}$ ), again in keeping with published findings [ $[\underline{25}]$. Finally, expression of TMPRSS2 isoform 1 markedly increased viral spread to levels observed in the presence of isoform 2 , and spread was markedly reduced by camostat mesylate (not shown), a serine protease inhibitor known to be active against TMPRSS2. In sum, these results indicate that isoform 1 and isoform 2 can efficiently cleave and activate FLUAV HA.

\section{TMPRSS2 isoform 1 cleaves and activates the spike protein of SARS- and MERS-coronavirus}

Apart from FLUAV, several respiratory viruses hijack TMPRSS2 to facilitate activation of their envelope glycoprotein, including the emerging SARS-CoV [ 14-16] and MERS-CoV $[\underline{29}, 30]$. Activation of SARS-S and MERS-S by TMPRSS2 isoform 2 occurs during viral entry into target cells and renders this process independent from the activity of cathepsin L $[\underline{14}-16, \underline{29}, \underline{30}]$, an endosomal cysteine protease which can activate SARS-S and MERS-S for entry into TMPRSS2-negative cells [29-31]. We investigated whether TMPRSS2 isoform 1 can also activate SARS-S and MERS-S. Western blot analysis of protease and SARS-S or MERS-S transfected cells revealed that both TMPRSS2 isoforms processed the S proteins comparably (Fig 5A and data not shown). Moreover, SARS-S- and MERS-S-driven entry into control cells was markedly reduced by pre-incubation of cells with MDL28170, a cathepsin B and L inhibitor, and this effect was rescued by TMPRSS2 isoform 2 and DESC1 but not TMPRSS3 (Fig 5B and $\underline{5 C})$, in keeping with published data $[\underline{14}-16,25,29, \underline{30}]$. Similarly, rescue was observed upon expression of isoform 1 (Fig $5 \mathrm{~B}$ and $5 \mathrm{C}$ ), indicating that the extended $\mathrm{N}$-terminus is compatible with coronavirus $\mathrm{S}$ protein activation during entry.

\section{Discussion}

Several host cell proteases can activate FLUAV HA in cell culture and it has thus been assumed that respiratory viruses can employ redundant proteolytic systems to ensure their activation in the host [4]. In stark contrast, recent studies indicated a central role for TMPRSS2 in viral 

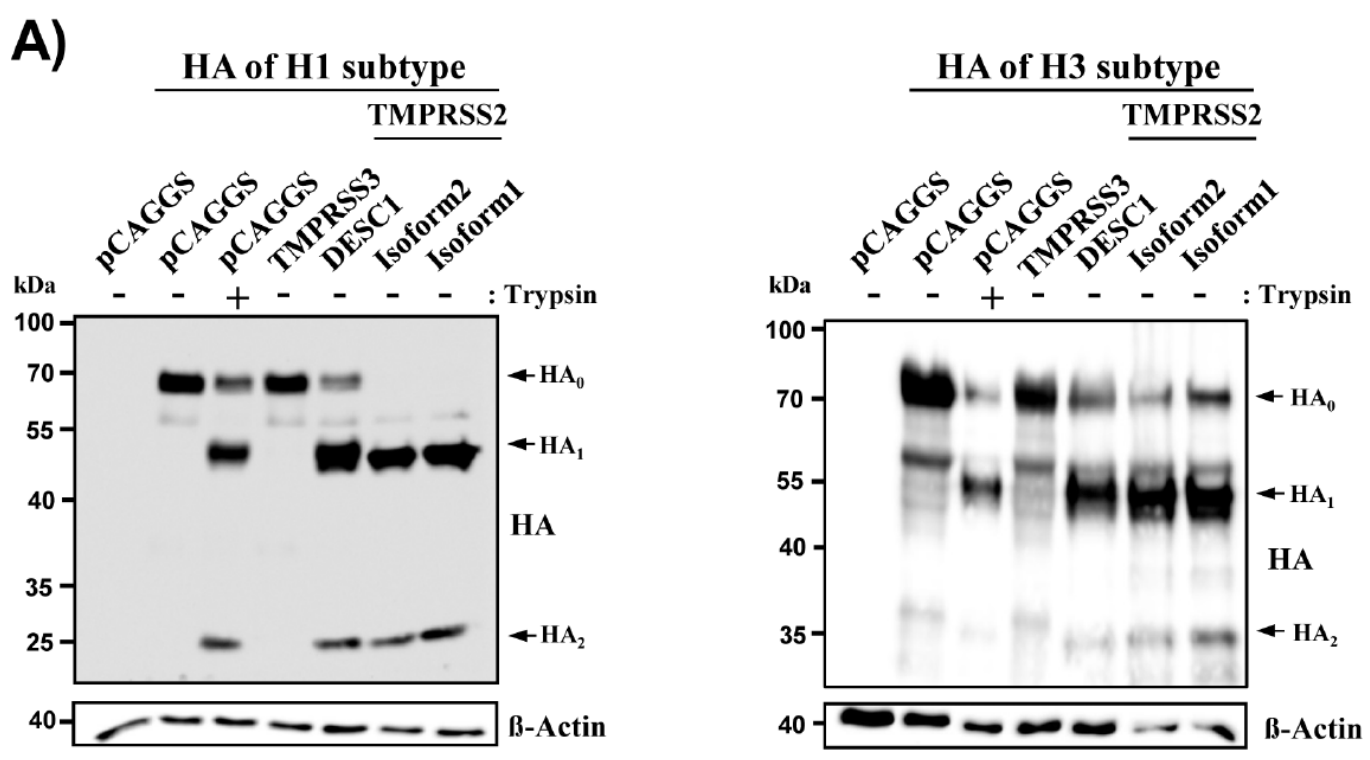

B)
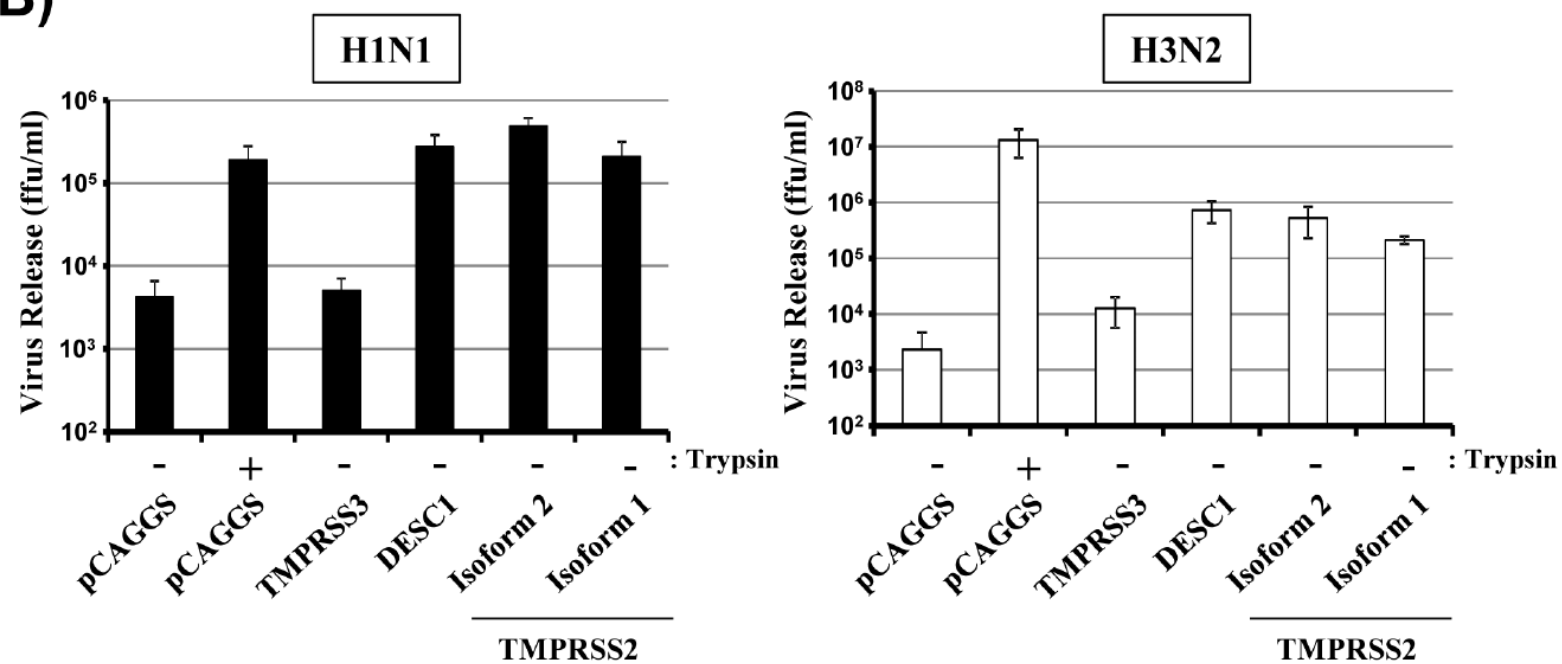

Fig 4. TMPRSS2 isoform 1 cleaves and activates the influenza virus hemagglutinin. (A) Expression plasmids encoding FLUAV HA subtypes $\mathrm{H} 1$ (left) and $\mathrm{H} 3$ (right) and the indicated proteases or empty plasmid (pCAGGS) were transiently cotransfected into $293 \mathrm{~T}$ cells. At $48 \mathrm{~h}$ post transfection the cells were treated with PBS or trypsin, and HA cleavage was determined by Western blotting. Similar results were obtained in three independent experiments. The $\mathrm{HA}_{0}$ precursor (upper arrow) and the $\mathrm{HA}_{1}$ (middle arrow) and $\mathrm{HA}_{2}$ (lower arrow) subunits are indicated. (B) The indicated proteases were transiently expressed in 293T cells and the cells infected with FLUAV A/PR/8/34 (H1N1) at an MOI 0.01 (left) or FLUAV A/Panama/2007/99 (H3N2) at an MOI of 0.1 (right) and treated with either trypsin or PBS. At $48 \mathrm{~h}$ post infection, the virus release was measured by determination of infectious particles (ffu/ml) in the culture supernatant. The results of representative experiments performed with triplicate samples are shown. Error bars indicate standard deviations. Similar results were obtained in three independent experiments. ffu, focus forming units.

doi:10.1371/joumal.pone.0138380.g004

activation: The protease was found to activate FLUAV and other respiratory viruses upon directed and upon endogenous expression in cell lines $[\underline{4}, \underline{6}-\underline{8}, \underline{14}-\underline{18}]$ and was shown to be expressed in viral target cells in the human aerodigestive tract [32]. Moreover, and more importantly, TMPRSS2 expression was required for spread and pathogenesis of FLUAV in 
A)

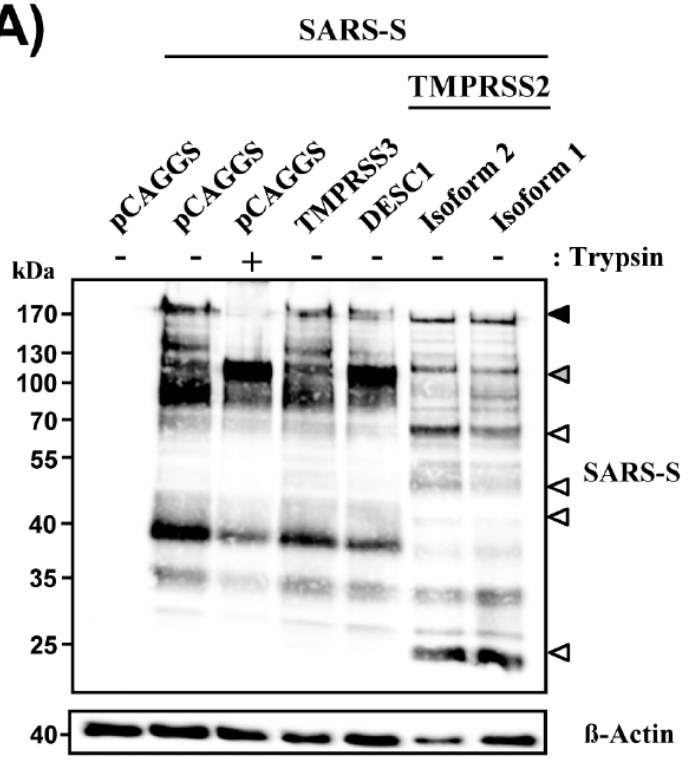

C)

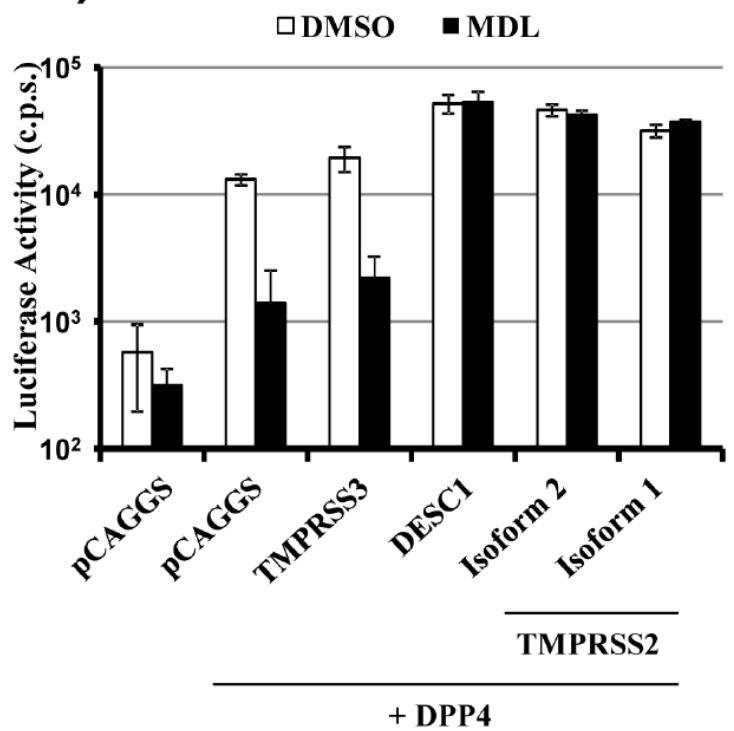

B)

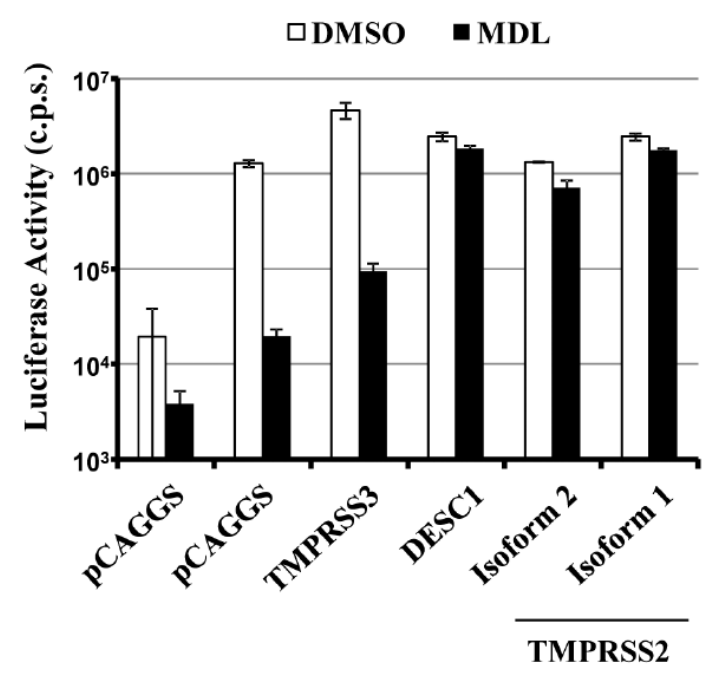

$+\mathrm{ACE} 2$

Fig 5. TMPRSS2 isoform 1 cleaves and activates the SARS-coronavirus spike protein. (A) 293T cells were cotransfected with plasmid encoding SARS-S with a C-terminal V5 tag and plasmids encoding the indicated proteases. At $48 \mathrm{~h}$ post transfection, the cells were treated with PBS or trypsin, and SARS-S cleavage was analyzed by Western blotting with a V5-specific antibody. The expression of $\beta$-actin served as a loading control. The results are representative of three independent experiments with different plasmid preparations. Black-filled arrowhead, uncleaved SARS-S; gray-filled arrowhead, S2 subunit generated by trypsin digest; white-filled arrowheads, C-terminal cleavage fragments generated by TMPRSS2. (B) To analyze SARS-S-driven viruscell fusion, 293T cells were transiently transfected with plasmids encoding the indicated proteases and ACE2. At $24 \mathrm{~h}$ post transfection, the cells were pretreated with medium supplemented with DMSO or $10 \mu \mathrm{M}$ cathepsin B/L inhibitor MDL 28170 , and transduced with pseudotypes bearing SARS-S. The luciferase activities in cell lysates were analyzed at $72 \mathrm{~h}$ post transduction. The results of a representative experiments performed with triplicate samples are shown. Error bars indicate standard deviations. Similar results were obtained in two separate experiments. (C) MERS-S-driven virus-cell fusion was analyzed as described for panel B but 293T cells transfected to express DPP4 were used as targets. The results of a representative experiment performed with triplicate samples are shown. Error bars indicate standard deviations. Similar results were obtained in three independent experiments. c.p.s., counts per second.

doi:10.1371/joumal.pone.0138380.g005 
mice [9-11]. Thus, TMPRSS2 is an important host factor for FLUAV and potentially other respiratory viruses and constitutes a potential target for antiviral intervention.

The tmprss 2 gene is located on chromosome 21 and comprises 14 exons and 13 introns $[\underline{23}, \underline{24}]$. It has been suggested that tmprss 2 mRNA may be alternatively spliced $[\underline{23}, \underline{24}]$ and two transcript variants were documented at NCBI, which only differ in the first exon. The encoded proteins are identical except for the presence of an extended cytoplasmic domain in isoform 1 and the impact of this extension on expression and function was unknown. The present study shows that isoform 1 is efficiently expressed in transfected cells and, in keeping with expectations, exhibits a slightly increased molecular weight relative to isoform 2 . Thus, the enzymatically inactive zymogen form of isoform 1 migrated slower than its counterpart in isoform 2 (the two bands observed might be the result of differential glycosylation) and differences were also observed for the $\mathrm{N}$-terminal fragment of the protease, which is generated upon autocatalytic activation. Notably, two prominent $\mathrm{N}$-terminal fragments were consistently observed for isoform 1 but not for isoform 2. Thus, the extended cytoplasmic domain might give rise to the expression of different glycoforms or, maybe more likely, might alter the specificity of autocatalytic cleavage, which is essential for the enzymatic activity of TMPRSS2. In fact, an impact of the cytoplasmic domain on the autocatalytic activation has previously been reported for the TTSP corin, in which the presence of a shortened cytoplasmic tail, due to the use of an alternative exon 1, reduces plasma membrane localization and autocatalytic activation [21].

An RT-PCR designed for the amplification of a sequence specific to transcript variant 1 showed that this transcript is expressed in cell lines and tissues susceptible to FLUAV infection and previously found to be positive for tmprss $2 \mathrm{mRNA}$ and or protein, including lung and liver $[\underline{23}, \underline{28}]$. In general, the expression patterns of transcript variant 1 and 2 were similar but not identical, suggesting that in certain tissues only isoform 1 might be available for viral activation. Whether the mRNA expression data match the expression of protein remains to be determined and such studies are complicated by the absence of isoform-specific antibodies. No major differences in the cellular localization of the two isoforms were observed, although distribution of isoform 2 at or close to the cell surface was more homogenous than that of isoform 1 , which was frequently detected within speckles. Thus, the extended cytoplasmic tail of isoform 1 slightly impacts protein localization in transfected cells and this impact might be more pronounced in cells endogenously expressing this isoform.

Our mRNA expression data suggest that at least a fraction of the TMPRSS2 molecules present in FLUAV target cells correspond to isoform 1, raising the question whether they contribute to viral activation. Our study provides an affirmative answer: Both isoform 1 and 2 colocalized with HA in transfected cells, cleaved HA upon coexpression and activated authentic FLUAV of different subtypes. Moreover, isoform 1, like isoform 2, activated SARS-S and MERS-S for entry into target cells. Thus, the presence of an extended $\mathrm{N}$-terminus does not seem to alter the ability of TMPRSS2 to activate respiratory viruses, although one should take into account that transfected cells might not mirror all aspects of cells endogenously expressing isoform 1.

Collectively, our results indicate that isoform 1 of TMPRSS2 can be produced in viral target cells and likely contributes to viral activation. Therefore, approaches to suppress HA activation for influenza therapy must target both isoforms and our initial results obtained with camostat mesylate indicate that this is feasible. However, it remains to be determined whether subtle differences in cellular localization and autocatalytic activation of TMPRSS2 isoforms observed here impact sensitivity to protease inhibitors in cells endogenously expressing these isoforms. 


\section{Acknowledgments}

We thank M. Winkler for helpful discussion.

\section{Author Contributions}

Conceived and designed the experiments: PZ HHW SP. Performed the experiments: PZ ASM. Analyzed the data: PZ SP. Contributed reagents/materials/analysis tools: HHW. Wrote the paper: PZ SP.

\section{References}

1. Taubenberger JK, Kash JC (2010) Influenza virus evolution, host adaptation, and pandemic formation. Cell Host Microbe 7: 440-451. doi: 10.1016/j.chom.2010.05.009 PMID: 20542248

2. Morens DM, Taubenberger JK, Harvey HA, Memoli MJ (2010) The 1918 influenza pandemic: lessons for 2009 and the future. Crit Care Med 38: e10-e20. doi: 10.1097/CCM.0b013e3181ceb25b PMID: $\underline{20048675}$

3. Edinger TO, Pohl MO, Stertz S (2014) Entry of influenza A virus: host factors and antiviral targets. J Gen Virol 95: 263-277. doi: 10.1099/vir.0.059477-0 PMID: 24225499

4. Bertram S, Glowacka I, Steffen I, Kühl A, Pöhlmann S (2010) Novel insights into proteolytic cleavage of influenza virus hemagglutinin. Rev Med Virol 20: 298-310. doi: 10.1002/mv.657 PMID: 20629046

5. Bertram S, Glowacka I, Blazejewska P, Soilleux E, Allen P, Danisch S, et al. (2010) TMPRSS2 and TMPRSS4 facilitate trypsin-independent spread of influenza virus in Caco-2 cells. J Virol 84: 1001610025. doi: 10.1128/JVI.00239-10 PMID: 20631123

6. Böttcher-Friebertshauser E, Stein DA, Klenk HD, Garten W (2011) Inhibition of influenza virus infection in human airway cell cultures by an antisense peptide-conjugated morpholino oligomer targeting the hemagglutinin-activating protease TMPRSS2. J Virol 85: 1554-1562. doi: 10.1128/JVI.01294-10 PMID: 21123387

7. Böttcher E, Matrosovich T, Beyerle M, Klenk HD, Garten W, Matrosovich M (2006) Proteolytic activation of influenza viruses by serine proteases TMPRSS2 and HAT from human airway epithelium. J Virol 80: 9896-9898. PMID: 16973594

8. Chaipan C, Kobasa D, Bertram S, Glowacka I, Steffen I, Tsegaye TS, et al. (2009) Proteolytic activation of the 1918 influenza virus hemagglutinin. J Virol 83: 3200-3211. doi: 10.1128/JVI.02205-08 PMID: 19158246

9. Hatesuer B, Bertram S, Mehnert N, Bahgat MM, Nelson PS, Pöhlmann S, et al. (2013) Tmprss2 Is Essential for Influenza H1N1 Virus Pathogenesis in Mice. PLoS Pathog 9: e1003774. doi: 10.1371/ journal.ppat.1003774 PMID: 24348248

10. Sakai K, Ami Y, Tahara M, Kubota T, Anraku M, Abe M, et al. (2014) The host protease TMPRSS2 plays a major role in in vivo replication of emerging $\mathrm{H} 7 \mathrm{~N} 9$ and seasonal influenza viruses. $\mathrm{J}$ Virol 88 5608-5616. doi: 10.1128/JVI.03677-13 PMID: 24600012

11. Tarnow C, Engels G, Arendt A, Schwalm F, Sediri H, Preuss A, et al. (2014) TMPRSS2 is a host factor that is essential for pneumotropism and pathogenicity of $\mathrm{H} 7 \mathrm{~N} 9$ influenza A virus in mice. $\mathrm{J}$ Virol 88: 4744-4751. doi: 10.1128/JVI.03799-13 PMID: 24522916

12. Cheng Z, Zhou J, To KK, Chu H, Li C, Wang D, et al. (2015) The identification of TMPRSS2 as the susceptible gene for severe illness of 2009 pandemic $A(H 1 N 1)$ influenza and infection of $A(H 7 N 9)$ influenza. J Infect Dis.

13. Bertram S, Dijkman R, Habjan M, Heurich A, Gierer S, Glowacka I, et al. (2013) TMPRSS2 activates the human coronavirus $229 \mathrm{E}$ for cathepsin-independent host cell entry and is expressed in viral target cells in the respiratory epithelium. J Virol 87:6150-6160. doi: 10.1128/JVI.03372-12 PMID: 23536651

14. Glowacka I, Bertram S, Müller MA, Allen P, Soilleux E, Pfefferle S, et al. (2011) Evidence that TMPRSS2 activates the severe acute respiratory syndrome coronavirus spike protein for membrane fusion and reduces viral control by the humoral immune response. J Virol 85: 4122-4134. doi: 10. 1128/JVI.02232-10 PMID: 21325420

15. Matsuyama S, Nagata N, Shirato K, Kawase M, Takeda M, Taguchi F (2010) Efficient activation of the severe acute respiratory syndrome coronavirus spike protein by the transmembrane protease TMPRSS2. J Virol 84: 12658-12664. doi: 10.1128/JVI.01542-10 PMID: 20926566

16. Shulla A, Heald-Sargent T, Subramanya G, Zhao J, Perlman S, Gallagher T (2011) A transmembrane serine protease is linked to the severe acute respiratory syndrome coronavirus receptor and activates virus entry. J Virol 85: 873-882. doi: 10.1128/JVI.02062-10 PMID: 21068237 
17. Abe M, Tahara M, Sakai K, Yamaguchi H, Kanou K, Shirato K, et al. (2013) TMPRSS2 is an activating protease for respiratory parainfluenza viruses. J Virol 87: 11930-11935. doi: 10.1128/JVI.01490-13 PMID: 23966399

18. Shirogane Y, Takeda M, Iwasaki M, Ishiguro N, Takeuchi H, Nakatsu Y, et al. (2008) Efficient multiplication of human metapneumovirus in Vero cells expressing the transmembrane serine protease TMPRSS2. J Virol 82: 8942-8946. doi: 10.1128/JVI.00676-08 PMID: 18562527

19. Esumi M, Ishibashi M, Yamaguchi H, Nakajima S, Tai Y, Kikuta S, et al. (2015) Transmembrane serine protease TMPRSS2 activates hepatitis C virus infection. Hepatology 61: 438-447.

20. Kim TS, Heinlein C, Hackman RC, Nelson PS (2006) Phenotypic analysis of mice lacking the Tmprss2encoded protease. Mol Cell Biol 26: 965-975. PMID: 16428450

21. Qi X, Jiang J, Zhu M, Wu Q (2011) Human corin isoforms with different cytoplasmic tails that alter cell surface targeting. J Biol Chem 286: 20963-20969. doi: 10.1074/jbc.M110.217570 PMID: 21518754

22. Lapointe J, Kim YH, Miller MA, Li C, Kaygusuz G, van de Rijn M, et al. (2007) A variant TMPRSS2 isoform and ERG fusion product in prostate cancer with implications for molecular diagnosis. Mod Pathol 20: 467-473. PMID: 17334351

23. Jacquinet E, Rao NV, Rao GV, Zhengming W, Albertine KH, Hoidal JR (2001) Cloning and characterization of the cDNA and gene for human epitheliasin. Eur J Biochem 268: 2687-2699. PMID: 11322890

24. Paoloni-Giacobino A, Chen H, Peitsch MC, Rossier C, Antonarakis SE (1997) Cloning of the TMPRSS2 gene, which encodes a novel serine protease with transmembrane, LDLRA, and SRCR domains and maps to 21q22.3. Genomics 44: 309-320. PMID: 9325052

25. Zmora P, Blazejewska P, Moldenhauer AS, Welsch K, Nehlmeier I, Wu Q, et al. (2014) DESC1 and MSPL activate influenza A viruses and emerging coronaviruses for host cell entry. J Virol 88: 1208712097. doi: 10.1128/JVI.01427-14 PMID: 25122802

26. Matthaei M, Budt M, Wolff T (2013) Highly pathogenic H5N1 influenza A virus strains provoke heterogeneous IFN-alpha/beta responses that distinctively affect viral propagation in human cells. PLoS One 8 : e56659. doi: 10.1371/journal.pone.0056659 PMID: 23451066

27. Wrensch F, Winkler M, Pöhlmann S (2014) IFITM proteins inhibit entry driven by the MERS-coronavirus spike protein: evidence for cholesterol-independent mechanisms. Viruses 6: 3683-3698. doi: 10.3390/ v6093683 PMID: 25256397

28. Vaarala MH, Porvari KS, Kellokumpu S, Kyllonen AP, Vihko PT (2001) Expression of transmembrane serine protease TMPRSS2 in mouse and human tissues. J Pathol 193: 134-140. PMID: 11169526

29. Gierer S, Bertram S, Kaup F, Wrensch F, Heurich A, Kramer-Kühl A, et al. (2013) The spike protein of the emerging betacoronavirus EMC uses a novel coronavirus receptor for entry, can be activated by TMPRSS2, and is targeted by neutralizing antibodies. J Virol 87: 5502-5511. doi: 10.1128/JVI.0012813 PMID: 23468491

30. Shirato K, Kawase M, Matsuyama S (2013) Middle East respiratory syndrome coronavirus infection mediated by the transmembrane serine protease TMPRSS2. J Virol 87: 12552-12561. doi: 10.1128/ JVI.01890-13 PMID: 24027332

31. Simmons G, Gosalia DN, Rennekamp AJ, Reeves JD, Diamond SL, Bates P (2005) Inhibitors of cathepsin L prevent severe acute respiratory syndrome coronavirus entry. Proc Natl Acad Sci U S A 102: 11876-11881. PMID: 16081529

32. Bertram S, Heurich A, Lavender H, Gierer S, Danisch S, Perin P, et al. (2012) Influenza and SARScoronavirus activating proteases TMPRSS2 and HAT are expressed at multiple sites in human respiratory and gastrointestinal tracts. PLoS One 7: e35876. doi: 10.1371/journal.pone.0035876 PMID: $\underline{22558251}$ 


\title{
Fourth manuscript
}

\section{Tetherin sensitivity of influenza A viruses is strain specific: Role of hemagglutinin and neuraminidases}

\author{
Gnirß, K.*, Zmora, P.*, Blazejewska, P., Winkler, M., Lins, A., Nehlmeier, I., \\ Gärtner, S., Moldenhauer, A.S., Hofmann-Winkler, H., Wolff, T., \\ Schindler, M., Pöhlmann, S. \\ *equal contribution
}

Journal of Virology, 2015, 89:9178-88; doi: 10.1128/JVI.01427-14

\section{Individual contribution:}

Analysis of the dose-dependence of the tetherin-mediated inhibition of the influenza virus release (Fig. 3A). Demonstration that tetherin blocks influenza virus release from MDCK cells (Fig. 3C). Knock-down of tetherin expression in a lung-derived cell line by siRNA (Fig. 4A) and analysis of its effects on influenza virus release (Fig. 4B). Demonstration that pandemic but not seasonal influenza viruses downregulate of tetherin expression in a hemagglutinin/neuraminidase-dependent fashion (Fig. 6A) and quantification and statistical analysis of the results (Fig. 6B). Analysis whether influenza viruses preferentially infect tetherin-positive/negative cells (Fig. R1 in rebuttal letter). Showing that tetherin expression does not modulate the efficiency of influenza virus cell entry (Fig. R2 in rebuttal letter). Writing parts of the manuscript (Material and Methods, Results) 


\title{
Tetherin Sensitivity of Influenza A Viruses Is Strain Specific: Role of Hemagglutinin and Neuraminidase
}

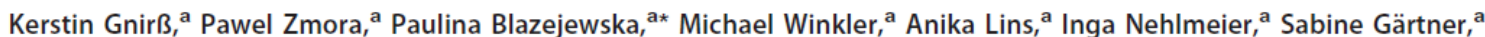 \\ Anna-Sophie Moldenhauer, ${ }^{a}$ Heike Hofmann-Winkler, ${ }^{a}$ Thorsten Wolff, ${ }^{\text {b }}$ Michael Schindler, ${ }^{\text {c,d }}$ (D) Stefan Pöhlmann ${ }^{\text {a }}$ \\ Infection Biology Unit, German Primate Center, Göttingen, Germanyª; Division of Influenza Viruses and other Respiratory Viruses, Robert Koch Institute, Berlin, Germany ${ }^{b}$; \\ Institute of Virology, Helmholtz Zentrum Munich, German Research Center for Environmental Health, Munich, Germany; Institute of Medical Virology and Epidemiology \\ of Viral Diseases, University Clinic Tübingen, Tübingen, Germany ${ }^{d}$
}

\section{ABSTRACT}

The expression of the antiviral host cell factor tetherin is induced by interferon and can inhibit the release of enveloped viruses from infected cells. The Vpu protein of HIV-1 antagonizes the antiviral activity of tetherin, and tetherin antagonists with Vpulike activity have been identified in other viruses. In contrast, it is incompletely understood whether tetherin inhibits influenza $\mathrm{A}$ virus (FLUAV) release and whether FLUAV encodes tetherin antagonists. Here, we show that release of several laboratoryadapted FLUAV strains and a seasonal FLUAV strain is inhibited by tetherin, while pandemic FLUAV A/Hamburg/4/2009 is resistant. Studies with a virus-like particle system and analysis of reassortant viruses provided evidence that the viral hemagglutinin (HA) is an important determinant of tetherin antagonism but requires the presence of its cognate neuraminidase (NA) to inhibit tetherin. Finally, tetherin antagonism by FLUAV was dependent on the virion context, since retrovirus release from tetherin-positive cells was not rescued, and correlated with an HA- and NA-dependent reduction in tetherin expression. In sum, our study identifies HA and NA proteins of certain pandemic FLUAV as tetherin antagonists, which has important implications for understanding FLUAV pathogenesis.

\section{IMPORTANCE}

Influenza A virus (FLUAV) infection is responsible for substantial global morbidity and mortality, and understanding how the virus evades the immune defenses of the host may uncover novel targets for antiviral intervention. Tetherin is an antiviral effector molecule of the innate immune system which can contribute to control of viral invasion. However, it has been unclear whether FLUAV is inhibited by tetherin and whether these viruses encode tetherin-antagonizing proteins. Our observation that several pandemic FLUAV strains can counteract tetherin via their HA and NA proteins identifies these proteins as novel tetherin antagonists and indicates that HA/NA-dependent inactivation of innate defenses may contribute to the efficient spread of pandemic FLUAV.

T: he interferon (IFN) system is an integral part of innate immunity $(1,2)$. Sensors of the IFN system recognize pathogenassociated molecular patterns and induce signaling cascades which induce the production and release of IFN. Binding of IFN to cell surface receptors elicits signals which induce the expression of IFN-stimulated genes (ISGs), some of which have antiviral activity $(3,4)$. A recently described ISG with antiviral activity is tetherin (CD317, BST-2). Tetherin's antiviral activity was identified in the context of HIV-1 infection, and it was shown that the viral protein $\mathrm{U}(\mathrm{Vpu})$ can antagonize tetherin $(5,6)$. Subsequently, it was demonstrated that several viruses are sensitive to inhibition by tetherin and that some of these viruses encode tetherin antagonists (7). Finally, recent studies indicate that tetherin can limit viral replication in the host $(8,9)$, underlining that tetherin is an important component of the innate defenses against viral invasion and can force viruses to install countermeasures.

The particular membrane topology of tetherin is key to its antiviral activity: Tetherin has membrane anchors at its $\mathrm{N}$ and $\mathrm{C}$ termini, which enable the protein to insert simultaneously into the viral envelope and the plasma membrane. As a consequence, tetherin forms a physical connector between virus and host cell which impedes the release of progeny virions into the extracellular space $(10,11)$. Tetherin exerts its antiviral activity at the plasma membrane, and most but not all virally encoded tetherin antagonists inactivate tetherin by reducing tetherin levels at the plasma membrane (7). For instance, the Vpu protein of HIV-1 targets tetherin for degradation in endosomes/lysosomes $(6,7,12-14)$ and interferes with transport of tetherin to the cell surface (6; reviewed in reference 7).

Influenza A viruses (FLUAV) cause annual epidemics (seasonal FLUAV) and intermittent pandemics (pandemic FLUAV) which are associated with substantial morbidity and mortality

Received 9 March 2015 Accepted 15 June 2015

Accepted manuscript posted online 24 June 2015

Citation Gnirß K, Zmora P, Blazejewska P, Winkler M, Lins A, Nehlmeier I, Gärtner S, Moldenhauer A-S, Hofmann-Winkler H, Wolff T, Schindler M, Pöhlmann S. 2015. Tetherin sensitivity of influenza A viruses is strain specific: role of hemagglutinin and neuraminidase. J Virol 89:9178-9188. doi:10.1128/JVI.00615-15.

Editor: D. S. Lyles

Address correspondence to Stefan Pöhlmann, spoehlmann@dpzeu

* Present address: Paulina Blazejewska, Boehringer Ingelheim Veterinary Research Center, Hannover, Germany.

K.G. and P.Z. contributed equally to this article.

Copyright $@ 2015$, American Society for Microbiology. All Rights Reserved.

doi:10.1128/JVI.00615-15

The authors have paid a fee to allow immediate free access to this article. 
(15). FLUAV are released from the plasma membranes of infected cells (16), the site where tetherin unfolds its antiviral activity, and thus either should be inhibited by tetherin or should encode tetherin antagonists. However, the role of tetherin in FLUAV infection has not been well defined. Initial studies indicated that FLUAV is only inefficiently inhibited by tetherin or is completely tetherin insensitive (17-19), while release of FLUAV-like particles is inhibited by tetherin (18). In contrast, subsequent analyses demonstrated appreciable inhibition of FLUAV release by tetherin (2022). In addition, evidence for a tetherin-antagonizing activity of certain neuraminidase (NA) proteins was reported $(20,23)$, but the antagonism is believed to be relatively inefficient (21). The surface proteins of several viruses can antagonize tetherin (2429), but so far whether combinations of hemagglutinin (HA) and NA can inhibit tetherin has not been examined systematically.

Here, we demonstrate in a FLUAV-based virus-like particle (VLP) system that the HA of the pandemic 1918 influenza virus rescues its corresponding NA from inhibition by tetherin, while the HA of the related, laboratory-adapted WSN virus fails to do so. Furthermore, we show that the HA and NA proteins of several pandemic FLUAV can rescue an $\mathrm{A} / \mathrm{PR} / 8 / 1934$ reassortant virus from inhibition by tetherin. Finally, we demonstrate that tetherin antagonism by $\mathrm{HA}$ and NA is dependent on the viral budding system and may involve reduction of tetherin expression.

\section{MATERIALS AND METHODS}

Cell culture. HEK 293T cells were maintained in Dulbecco's modified Eagle's medium (DMEM) (Pan Biotech) supplemented with 10\% fetal calf serum (Biochrom) and $1 \%$ penicillin-streptomycin (Pan Biotech) in a humidified atmosphere with $5 \% \mathrm{CO}_{2}$ at $37^{\circ} \mathrm{C}$. MDCK and NCI-H358 cells (30) were cultured under the same conditions using minimum essential medium (MEM) (Gibco) or Roswell Park Memorial Institute (RPMI) 1640 medium (Pan Biotech), respectively, supplemented with $10 \%$ fetal calf serum and penicillin-streptomycin.

Plasmids. The plasmids encoding HIV-1 Vpu (31), human tetherin (32), 1918 HA (A/South Carolina/1/1918)/), 1918 NA (A/Brevig Mission/ 1/1918) with and without a C-terminal V5 tag (33), WSN HA (A/WSN/ 1933), WSN NA (A/WSN/1933) (33), and HIV-1 p96ZM651gag-opt (34) were described previously. Expression plasmids for WSN HA and NA with C-terminal V5-antigenic tags were constructed by PCR using the primers $5^{\prime}$-GCGAATTCGCATGAATCCAAACCAGAAAATAATAACC ATTGG- $3^{\prime}$ and $3^{\prime}$-GCCTCGAGGCCTACGTAGAATCGAGACCGAGG AGAGGGTTAGGGATAGGCTTACCCTTGTCAATGG-5' (WSN NA) and $5^{\prime}$-GCGGGTACCACCATGAAGGCAAAACTACTGGTCCTGT TATATGCATTTGTAGCTACAGATGCAGACACAATATGTATAGGC TACC- $3^{\prime}$ and $3^{\prime}$-GCCTCGAGGCTCACGTAGAATCGAGACCGAGG AGAGGGTTAGGGATAGGCTTACCGATGCATATTCTGCACTG CAAAGACCCATT-5' (WSN HA). PCR-generated sequences were cloned into pCAGGS via EcoRI/XhoI (WSN NA-V5) and KpnI/Xhol (WSN HA-V5). For the generation of a retroviral vector encoding tetherin, the tetherin sequence was PCR amplified using primers hTetherin-5B (5' -GGGATCCACCATGGCATCTACTTCGTATG-3') and hTetherin-3X (5'-GGCTCGAGTCACTGCAGCAGAGCGCTGA G-3') and cloned into pQCXIP-mcs using BamHI and XhoI. pQCXIPmcs is a modified version of pQCXIP (Clontech) in which the original polylinker has been replaced by a linker containing restriction sites for NotI, BamHI, AgeI, HpaI, MluI, XhoI, NruI, and EcoRI, employing the oligonucleotides linkQCXIPr (5'-CGGAATTCTCGC GACTCGAGACGCGTGTTAACACCGGTGGATCCGCGGCCGCGG$\left.3^{\prime}\right)$ and linkQCXIPf (5' -CCGCGGCCGCGGATCCACCGGTGTTAACA CGCGTCTCGAGTCGCGAGAATTCCG-3'). The integrity of all PCRamplified sequences was verified by automated sequencing.
Immunoblotting. For analysis of protein expression by Western blotting, samples were separated by $12.5 \%$ SDS-PAGE and subsequently transferred onto nitrocellulose membranes (Hartenstein) $(0.2 \mu \mathrm{m})$. Membranes were blocked with $5 \%$ milk powder-phosphate-buffered saline (PBS) plus $0.1 \%$ Triton X-100 (PBS-T). Incubation with the first antibody was performed overnight at $4^{\circ} \mathrm{C}$, while the secondary antibody was applied at room temperature (RT) for $1 \mathrm{~h}$. Subsequently, membranes were washed three times for $5 \mathrm{~min}$ with PBS-T. A commercially available ECL kit (ECL Prime Western blotting detection reagents; GE Healthcare) was used to detect bound antibodies, and signals were visualized by employing the imaging system ChemoCam with the ChemoStarProfessional software (Intas). For quantification of signal intensity, the ImageJ program was used (35).

Antibodies. For protein detection by Western blotting, the following antibodies were used: mouse anti-V5 antibody (Invitrogen) at a 1:2,500 dilution, rabbit anti- $\beta$-actin antibody (Abnova) at a 1:1,000 dilution, rabbit anti-tetherin antibody (NIH AIDS Research and Reference Reagent Program) at a dilution of 1:1,000, rabbit anti-tetherin antibody (Abcam) at a dilution of 1:1,000, anti-p24 hybridoma cell culture supernatant (183H12-5C) for detection of Gag at a dilution of 1:500, goat anti-FLUAV (Chemicon) at a dilution of 1:500, and rabbit anti-H3 (Immune Technology Corp) at a dilution of 1:1,000. As secondary antibodies for Western blotting, horseradish peroxidase (HRP)-coupled antibodies against mouse, goat, or rabbit (Dianova) were used at a 1:10,000 dilution. For focus formation assay, FLUAV was detected with a goat anti-FLUAV antibody (Virostat) at a 1:1,000 dilution. The anti-goat-HRP antibody (Dianova) used for immunoblotting served also as the secondary antibody in the focus formation assay but at a dilution of 1:1,000.

Recombinant viruses. The PR8-derived $6+2$ reassortant virus expressing the HA and NA surface glycoproteins of A/California/7/2009 (H1N1pdm) was generated through reverse genetic methods by transfection of RNA polymerase I-based expression vectors as described elsewhere (36). The HA protein of the PR $8 \times 2009 \mathrm{HA} / \mathrm{NA}$ reassortant expresses at HA position 225 a D-to-G substitution, which is a natural variation occurring in H1N1pdm strains. The PR8 $\times 1968$ HA/NA (X-31 virus) is a reassortant virus containing the HA and NA segments of A/Aichi/2/1968 (H3N2) while the remaining segments stem from A/PR/8/1934 (H1N1) (37).

Virus production in hen's eggs. A/PR/8/1934 (H1N1), A/WSN/1933 (H1N1), A/Panama/2007/1999 (H3N2), PR8 × 2009 HA/NA, and PR8 $\times$ $1968 \mathrm{HA} / \mathrm{NA}$ were propagated in the chorio-allantoic cavities of 10-dayold embryonated hen eggs (Valo BioMedia GmbH, Germany) for $48 \mathrm{~h}$ at $37^{\circ} \mathrm{C}$. A/Panama/2007/99 (H3N2) was reconstituted from an 8-plasmid system (38) before amplification in eggs.

Production of virus-like particles. For production of FLUAV-like particles, 293T cells were seeded in 6-well plates at a density of $2.8 \times 10^{5}$ cells/well and cotransfected with a plasmid encoding NA and either a plasmid encoding HA or empty plasmid in conjunction with a plasmid encoding tetherin or empty control plasmid. For production of HIV-like particles, 293T cells were seeded in 6-well or 12-well plates and cotransfected with a plasmid encoding codon-optimized Gag and either HA- or NA-encoding plasmids or both plasmids in the presence or absence of a tetherin-encoding plasmid or an empty control plasmid. At 12 to $16 \mathrm{~h}$ posttransfection, the medium was changed, and at $40 \mathrm{~h}$ posttransfection, $2 \mathrm{ml}$ supernatant per well of a 6 -well plate or $1 \mathrm{ml}$ of supernatant per well of a 12-well plate was harvested and cleared from debris by centrifugation at $1,500 \times g$ and $4^{\circ} \mathrm{C}$ for $5 \mathrm{~min}$. Subsequently, $1.4 \mathrm{ml} / 0.7 \mathrm{ml}$ (for 6-well/ 12 -well plates) supernatant was loaded onto a $20 \%$ sucrose cushion in TNE buffer and centrifuged at $17,000 \times g$ for $2 \mathrm{~h}$ at $4^{\circ} \mathrm{C}$. After discarding the supernatant, the pelleted VLPs were mixed with $30 \mu \mathrm{l} 2 \times$ SDS-containing lysis buffer (50 mM Tris [pH 6.8], 10\% glycerol, $2 \%$ SDS, $5 \%$ $\beta$-mercaptoethanol, $0.1 \%$ bromphenol blue, $1 \mathrm{mM} \mathrm{EDTA}$ ) and boiled for $20 \mathrm{~min}$ at $95^{\circ} \mathrm{C}$. Cell lysates were collected in $200 \mu \mathrm{l} / 100 \mu \mathrm{l}$ (for 6-well/ 12-well plates) $2 \times$ SDS lysis buffer/well and also boiled for $30 \mathrm{~min}$ at $95^{\circ} \mathrm{C}$. Finally, all samples were subjected to immunoblot analysis. 
Infection of tetherin-expressing cells with FLUAV. For infection experiments, 293T cells were seeded in 6-well plates at a density of $2.8 \times 10^{5}$ cells/well. After $24 \mathrm{~h}$, the cells were transfected with either a constant amount $(6 \mu \mathrm{g})$ or increasing amounts $(0.5,1.0,2.0,4.0$, or $6.0 \mu \mathrm{g})$ of tetherin expression plasmid, angiotensin-converting enzyme 2 (ACE2) encoding plasmid, or empty plasmid as control, employing the calcium phosphate transfection method. The transfection medium was replaced by fresh culture medium after an overnight incubation. At $24 \mathrm{~h}$ posttransfection, the culture medium was removed and the cells were washed gently with PBS and then incubated with Dulbecco's PBS (DPBS) supplemented with $\mathrm{Ca}^{2+}, \mathrm{Mg}^{2+}$, and $0.2 \%$ bovine serum albumin (BSA) and containing A/PR/8/1934 (H1N1) at a multiplicity of infection (MOI) of 0.1 or 0.01 , A/Panama/2007/1999 (H3N2) at an MOI of 0.3, A/WSN/1933 (H1N1) at an MOI of $0.01, \mathrm{~A} / \mathrm{Hamburg} / 4 / 2009(\mathrm{H} 1 \mathrm{~N} 1)$ at an MOI of $0.3, \mathrm{PR} 8 \times$ $2009 \mathrm{HA} / \mathrm{NA}$ at an MOI of 0.01 , or PR8 $\times 1968 \mathrm{HA} / \mathrm{NA}$ at an MOI of 0.01 . After $1 \mathrm{~h}$ of incubation at $37^{\circ} \mathrm{C}$, the infection medium was removed, the cells were gently washed with PBS, and fresh infection medium (DMEM supplemented with $0.2 \%$ bovine serum albumin and $1 \%$ penicillin-streptomycin) was added. Finally, the culture supernatants were collected at 24 $\mathrm{h}$ postinfection and cleared from debris by centrifugation, and the amount of infectious units present in the supernatants was determined by focus formation assay. To assess tetherin's antiviral activity in MDCK cells, cells were seeded in 12 -well plates at a density of $1.4 \times 10^{5}$ cells/well. After $24 \mathrm{~h}$ of incubation, the cells were transduced with a murine leukemia virus (MLV) vector encoding human tetherin or transduced with an empty control vector, as described previously (39). At $24 \mathrm{~h}$ postransduction, the cells were infected with A/PR/8/1934 (H1N1) or A/Hamburg/4/ $2009(\mathrm{H} 1 \mathrm{~N} 1)$ at an MOI of $0.01,0.1$, or 1.0 and processed as described above.

Knockdown of tetherin expression in NCI-H358 cells and FLUAV infection. The NCI-H358 cells were seeded in 12-well plates at a density of $1.1 \times 10^{5}$ cells/well. After $24 \mathrm{~h}$, the cells were transfected with $1 \mu \mathrm{g}$ of small interfering RNA (siRNA) specific for tetherin or with scrambled, nonsense siRNA as a control (Santa Cruz Biotechnology), using Lipofectamine 2000 (Life Technologies) according to the manufacturer's protocol. The transfection medium was replaced by fresh RPMI medium at $36 \mathrm{~h}$ posttransfection. At $48 \mathrm{~h}$ posttransfection, either the cells were infected as described below or tetherin expression was examined by Western blotting. For this, the cells were gently washed with PBS, collected in 100 $\mu \mathrm{l}$ of $2 \times$ SDS lysis buffer/well, and boiled for $30 \mathrm{~min}$ at $95^{\circ} \mathrm{C}$. Finally, all samples were subjected to immunoblot analysis, as described above.

For FLUAV infection, the culture medium was removed, and the cells were gently washed with prewarmed DPBS with $\mathrm{Ca}^{2+}, \mathrm{Mg}^{2+}$, and $0.2 \%$ BSA and then incubated with DPBS containing $\mathrm{Ca}^{2+}, \mathrm{Mg}^{2+}, \mathrm{BSA}$, and $\mathrm{A} / \mathrm{PR} / 8 / 1934$ (H1N1) at an MOI of 0.1, A/Hamburg/4/2009 (H1N1) at an MOI of 0.3 , PR $8 \times 2009 \mathrm{HA} / \mathrm{NA}$ at an MOI of 0.1 , or PR $8 \times 1968 \mathrm{HA} / \mathrm{NA}$ at an MOI of 0.1 . After $1 \mathrm{~h}$ of incubation at $37^{\circ} \mathrm{C}$, the medium containing viruses was removed, the cells were gently washed with prewarmed DPBS with $\mathrm{Ca}^{2+}, \mathrm{Mg}^{2+}$, and BSA, and fresh infection medium was added. The virus titers in the culture supernatant were determined at $24 \mathrm{~h}$ postinfection by focus formation assay.

Impact of FLUAV infection on tetherin-mediated inhibition of the release of HIV-like particles. To monitor the impact of FLUAV on tetherin-mediated restriction of release of HIV Gag-based VLPs, 293T cells were transfected to produce VLPs in the presence and absence of tetherin, as described above. At $16 \mathrm{~h}$ posttransfection, the cells were infected with A/Hamburg/4/2009 (H1N1) at an MOI of 1 and with PR $8 \times 1968 \mathrm{HA} / \mathrm{NA}$ at an MOI of 0.1 or 1 , and the cultures were processed as described above. Release of Gag-VLPs in the culture supernatants and expression of Gag, tetherin, and FLUAV proteins in cell lysates were determined at $24 \mathrm{~h}$ postinfection by immunoblotting, as described above.

Impact of FLUAV infection on tetherin expression. In order to determine the impact of FLUAV infection on tetherin expression, 293T cells were transiently transfected with $2 \mu \mathrm{g}$ of plasmid encoding tetherin or with empty plasmid as a control and subsequently infected with $\mathrm{A} / \mathrm{PR} / 8$ /
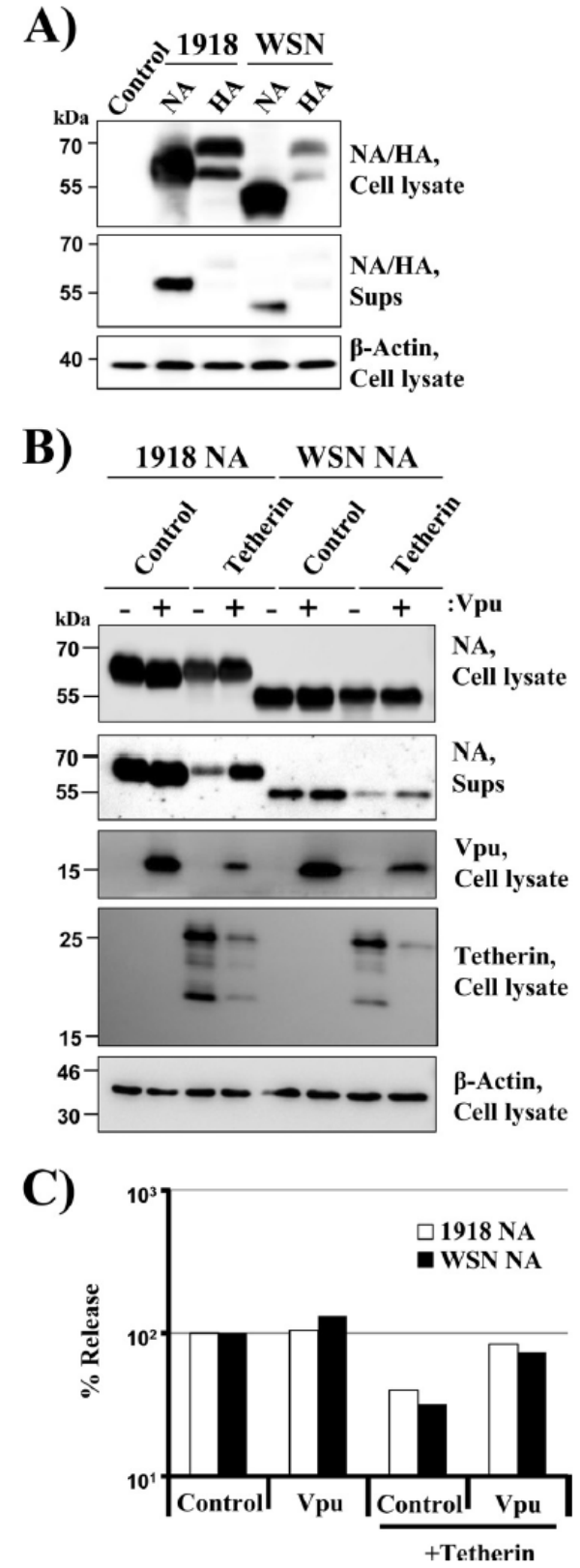

FIG 1 Tetherin inhibits release of neuraminidase-based FLUAV-like particles. (A) 293T cells were transfected with expression plasmids encoding the indicated NA and HA proteins with a C-terminal V5 antigenic tag or transfected with empty plasmid (control). At $40 \mathrm{~h}$ posttransfection, HA and NA expression in cell lysates and supernatants was detected via Western blotting, using a V5-specific antibody. Tetherin and Vpu expression was visualized using antibodies raised against these proteins. Detection of $\beta$-actin expression in cell lysates served as loading control. Similar results were obtained in at least four independent experiments. (B) The experiment was carried out as described for panel A, but cells were additionally cotransfected with an empty plasmid (control) or tetherin-encoding plasmid. In addition, a Vpu-encoding plasmid was cotransfected as indicated. The results of a representative experiment are shown and were confirmed in six separate experiments. (C) The signal intensities measured for panel B were quantified using Image J software. The NA signal present in supernatants was normalized to NA in cell lysates, and normalized NA signals in supernatants measured in the absence of tetherin and Vpu were set as 100\%. 1918, pandemic 1918 FLUAV; WSN, A/WSN/ 1933; HA, hemagglutinin; NA, neuraminidase; Sups, supernatants. 
A)

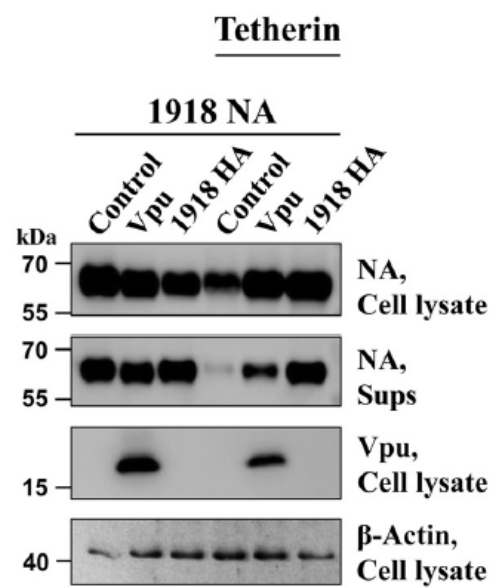

B) Tetherin

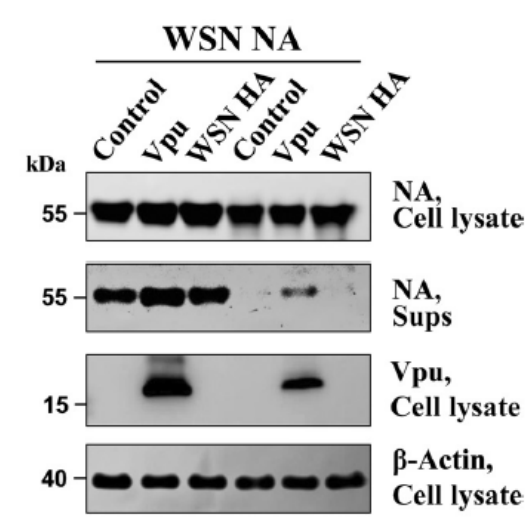

\section{C)}

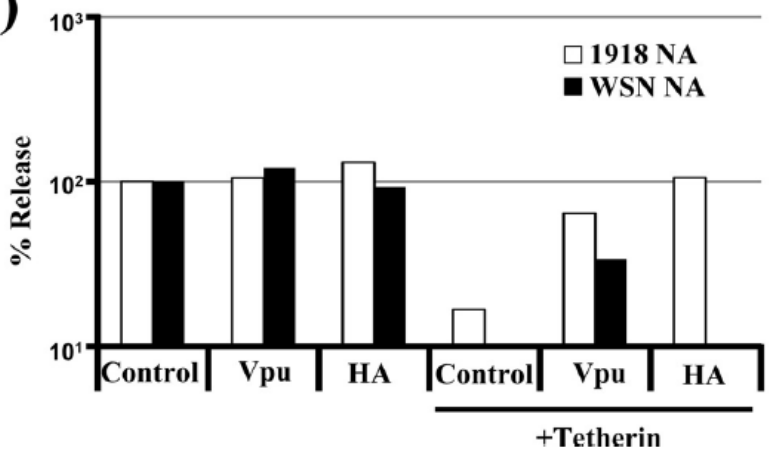

FIG 2 The hemagglutinin of pandemic 1918 influenza virus antagonizes tetherin. (A and B) 293T cells were cotransfected with expression plasmids encoding 1918 NA (A) or WSN NA (B) and empty plasmid or tetherin-encoding plasmid. In addition, cells were transfected with plasmids encoding the indicated HA proteins or Vpu or were transfected with an empty plasmid (control). At $40 \mathrm{~h}$ posttransfection, NA expression in cell lysates and supernatants was detected via Western blotting, using a V5-specific antibody. In addition, expression of Vpu in cell lysates was monitored. Detection of $\beta$-actin expression in cell lysates served as loading control. The results of representative experiments are shown and were confirmed in at least five (A) and three (B) separate experiments. (C) The signal intensities measured for panels A and B were quantified using ImageJ software. The NA signal present in supernatants was normalized to NA in cell lysates, and normalized NA signals in supernatants measured in the absence of tetherin and a tetherin antagonist were set as $100 \%$.

1934 (H1N1) at an MOI of 0.1, A/Hamburg/4/2009 (H1N1) at an MOI of 0.3 , PR $8 \times 2009 \mathrm{HA} / \mathrm{NA}$ at an MOI of 0.1 , or PR $8 \times 1968 \mathrm{HA} / \mathrm{NA}$ at an MOI of 0.1 , as described above. At $24 \mathrm{~h}$ postinfection, the cells were harvested for Western blot analysis of tetherin expression, as described above. Finally, Western blot images were analyzed with ImageJ software to quantify tetherin expression.

Focus formation assay. The amount of infectious units within the culture supernatants was determined by focus formation assay, as described previously $(19,40)$. In brief, serial $2-, 5-$, or 10 -fold dilutions of samples were prepared in infection medium (DMEM with $1 \%$ penicillinstreptomycin and $0.1 \%$ BSA) and were titrated on MDCK cells. After $1 \mathrm{~h}$ of incubation, the medium was replaced with infection medium containing an Avicel overlay and $2.5 \mu \mathrm{g} / \mathrm{ml} \mathrm{N}$-acetylated trypsin (Sigma). After an incubation period of $24 \mathrm{~h}$, the cells were fixed with $4 \%$ formalin in PBS and incubated for $1 \mathrm{~h}$ with anti-FLUAV polyclonal goat antibody (Virostat). Subsequently, the cells were washed, incubated for $1 \mathrm{~h}$ with antigoat-HRP antibodies (Dianova), washed, and incubated for $10 \mathrm{~min}$ with True Blue substrate (KPL). Finally, foci were counted, and viral titers were calculated as focus formation units (FFU) per ml of culture supernatant.

Statistical analysis. Results were analyzed for statistical significance using the Student $t$ test in the QuickCalcs GraphPad software (nonsignificant [NS], $P>0.05$;,$P<0.05$; $* *, P<0.01$; ${ }^{* * *}, P<0.005$ ).

\section{RESULTS}

Tetherin inhibits release of neuraminidase-based virus-like particles. The glycoproteins of several viruses were identified as tetherin antagonists (24-29). In order to address whether the NA and HA proteins of FLUAV exert tetherin-antagonizing activity, we employed an NA-based VLP system, which was previously used to analyze release restriction by tetherin (23). For this, the NA proteins of the pandemic virus A/Brevig Mission/1/1918 (abbreviated 1918) and the related, laboratory-adapted A/WSN/1933 (abbreviated WSN) were equipped with a V5 antigenic tag and transiently expressed in $293 \mathrm{~T}$ cells, followed by detection of NA expression in cell lysates and supernatants. The corresponding HA proteins, also harboring a V5 tag, were analyzed in parallel. Both NA proteins were comparably expressed in transfected cells and facilitated release of VLPs, but release was more efficient from cells expressing 1918 NA than for those expressing WSN NA (Fig. 1A). Expression of $1918 \mathrm{HA}$ in cell lysates was higher than expression of WSN HA, and none of the HA proteins tested was efficiently released in the extracellular space, which may be due to lack of budding capacity and/or retention of HA-based VLPs at the cell surface, as a consequence of HA binding to cellular factors modi- 


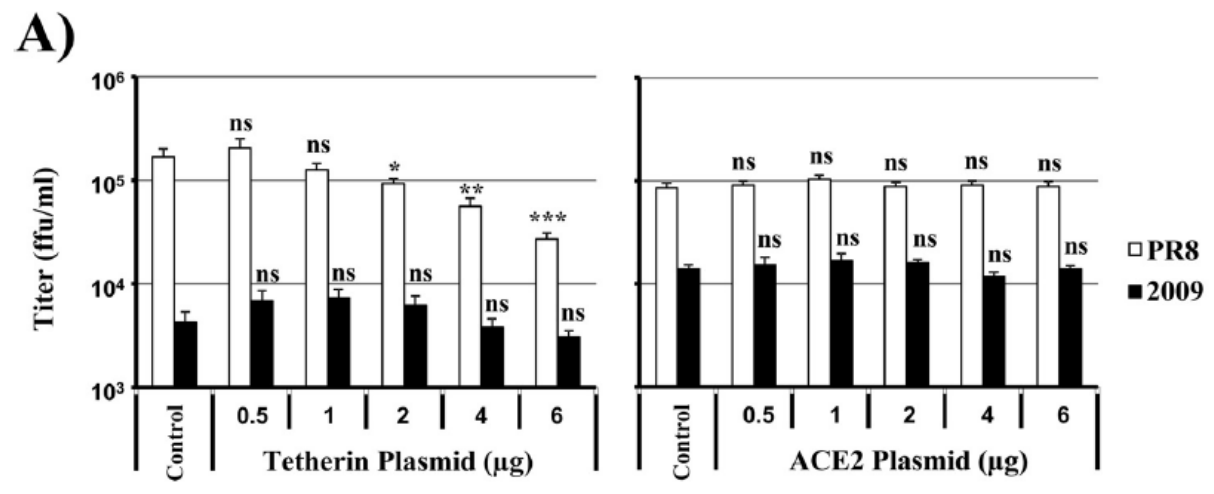

B)

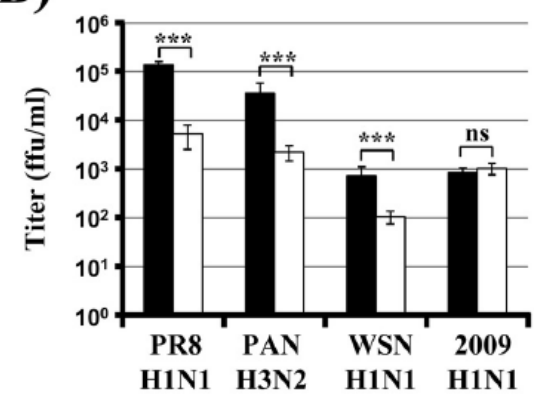

C)

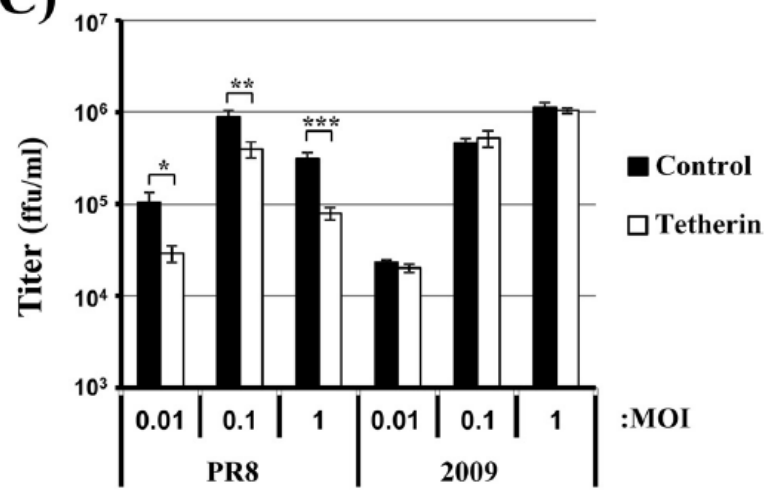

D)

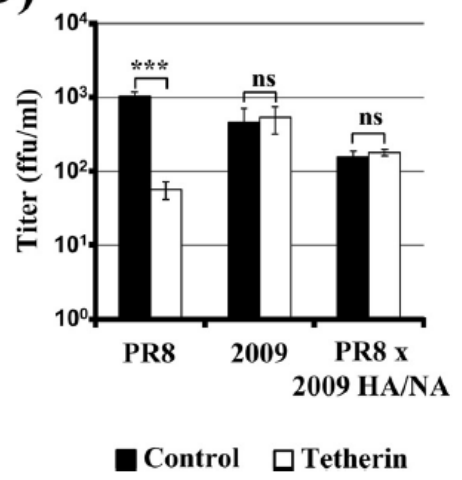

E)

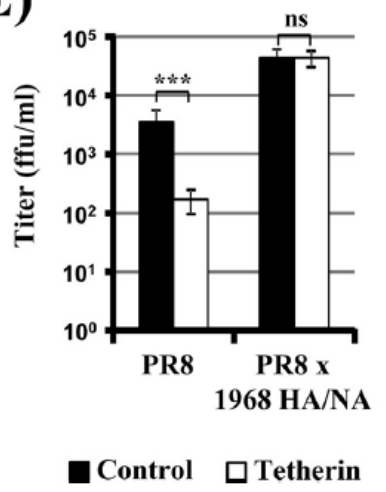

FIG 3 Neuraminidase and hemagglutinin of pandemic FLUAV confer resistance to tetherin in transfected cells. (A) Empty plasmid (control) or the indicated amounts of tetherin (left panel)- or angiotensin-converting enzyme 2 (ACE2) (right panel)-encoding plasmids were transfected into 293T cells, and the cells were infected with A/PR/8/1934 (H1N1) (abbreviated PR8) or A/Hamburg/4/2009 (H1N1) (abbreviated 2009) at MOIs of 0.1 and 0.3, respectively. At $1 \mathrm{~h}$ postinfection, the input viruses were removed by washing, and at $24 \mathrm{~h}$ postinfection, the supernatants were harvested and titers determined using the focus formation assay. The results of a single, representative experiment performed with triplicate samples are shown and were confirmed in three separate experiments. The statistical significance of differences between infection of control cells and tetherin- or ACE2-expressing cells was assessed employing Student's $t$ test. (B) 293T cells transfected with a plasmid encoding tetherin or transfected with empty plasmid (control) were infected with laboratory-adapted FLUAV PR8 and A/WSN/1933 (H1N1) (abbreviated WSN), seasonal FLUAV A/Panama/2007/1999 (H3N2) (abbreviated PAN), or pandemic FLUAV 2009 at an MOI of 0.1 (PR8), 0.3 (PAN and 2009), or 0.01 (WSN) for 1 h. Subsequently, cells were processed and viral titers determined as described for panel A. The results of a single experiment performed with quintuplicate samples are shown. Error bars indicate standard deviations (SD). Similar results were obtained in two separate experiments. (C) MDCK cells transduced to express tetherin or control transduced were infected with PR8 or 2009 at an MOI of 0.01, 0.1, or 1.0 and processed as described for panel B. The results of a single experiment performed with triplicate samples are shown and were confirmed in three separate experiments. Error bars indicate SD. (D and E) Infection was carried out as described for panel B, using wt PR8 and reassortant PR8 viruses equipped with HA and NA of 2009 (PR8 × 2009 HA/NA) (D) or A/Aichi/2/1968 (H3N2) (PR8 X $1968 \mathrm{HA} / \mathrm{NA}$ ) (E) at an MOI of 0.01. The results of single, representative experiments performed with sextuplicate samples are shown and were confirmed in a separate experiment. FFU, focus-forming units/ml; MOI, multiplicity of infection. 

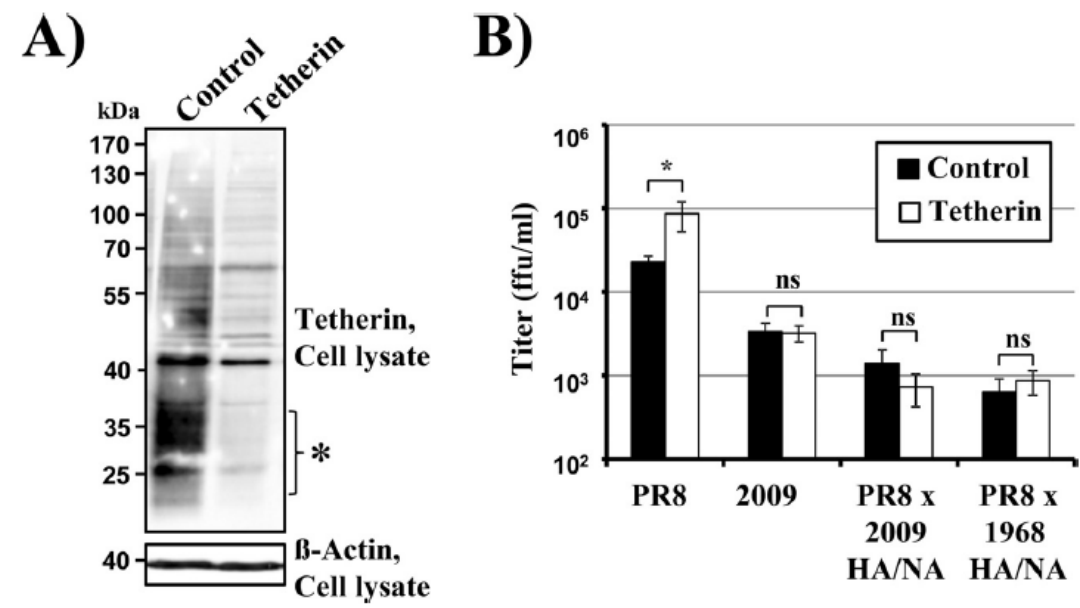

FIG 4 Neuraminidase and hemagglutinin of pandemic FLUAV confer resistance to endogenous tetherin in NCI-H358 cells. (A) NCI-H358 cells were transfected with scrambled siRNA (control) or tetherin-specific siRNA, and tetherin expression was assessed by Western blotting. The results of a representative experiment are shown and were confirmed in three separate experiments. Bands corresponding to tetherin are highlighted by an asterisk. The tetherin signal is heterogeneous, due to heterogeneity in tetherin glycosylation. (B) NCI-H358 cells were transfected with scrambled siRNA (control) or tetherin-specific siRNA and subsequently infected with A/PR/8/1934 (H1N1) (abbreviated PR8), A/Hamburg/4/2009 (H1N1) (abbreviated 2009), and a reassortant PR8 virus equipped with HA and NA of $2009(\mathrm{PR} 8 \times 2009 \mathrm{HA} / \mathrm{NA})$ or A/Aichi/2/1968 (H3N2) $(\mathrm{PR} 8 \times 1968 \mathrm{HA} / \mathrm{NA})$ at an MOI of $0.1(\mathrm{PR} 8, \mathrm{PR} 8 \times 2009 \mathrm{HA} / \mathrm{NA}$, and PR8 $\times 1968 \mathrm{HA} / \mathrm{NA})$ or 0.3 (2009). The average from three independent experiments performed with triplicates samples is shown. Error bars indicate standard error of the mean (SEM).

fied with 2,6-linked sialic acid (41). In order to address whether release of NA-based particles is suppressed by tetherin, we performed the VLP production as described above but coexpressed tetherin. Release of both 1918 NA- and WSN NA-based VLPs was markedly reduced upon tetherin expression, and this effect was rescued upon coexpression of HIV-1 Vpu (Fig. 1B and C), which diminished tetherin levels in the transfected cells, as expected (1214). These results indicate that release of 1918 NA- and WSN NA-based VLPs is readily detectable and sensitive to inhibition by tetherin. The NA-based VLP assay thus provides a suitable tool for the analysis of a potential tetherin-antagonizing activity of HA.

The hemagglutinin of the pandemic 1918 influenza virus antagonizes tetherin. We next analyzed whether the 1918 HA and WSN HA proteins can rescue VLP release driven by their cognate NAs from inhibition by tetherin. The tetherin-mediated release inhibition of 1918 NA-based VLPs was rescued by expression of both Vpu and 1918 HA (Fig. 2A), and tetherin counteraction by Vpu but not 1918 HA was associated with a reduction in tetherin expression (not shown). In contrast, release of WSN NA-VLPs from tetherin-positive cells was facilitated by Vpu but not WSN HA (Fig. 2B and C). Finally, 1918 HA failed to consistently rescue the release of WSN NA-based VLPs from inhibition by tetherin (not shown), suggesting that tetherin antagonism by HA requires the presence of the corresponding NA. Collectively, these results indicate that the HA of the pandemic 1918 influenza virus but not the HA of the laboratory-adapted WSN can antagonize tetherin in the presence of its cognate NA.

The 2009 pandemic influenza virus is resistant to tetherin. Our findings with the VLP system raised the possibility that seasonal and/or laboratory-adapted FLUAV (WSN was obtained upon repeated passage of a seasonal virus in animals) might be more sensitive to inhibition by tetherin than pandemic FLUAV. To investigate this, we infected tetherin-transfected 293T cells, removed input virus by washing, and assessed FLUAV release in culture supernatants. All experiments were conducted in the ab- sence of exogenously added trypsin, in order to ensure tetherin integrity and to prevent multicycle FLUAV spread. First, we expressed in 293T cells increasing amounts of tetherin or an irrelevant membrane protein, the severe acute respiratory syndrome (SARS) coronavirus entry receptor angiotensin-converting enzyme 2 (ACE2), and then infected the cells with either pandemic FLUAV (A/Hamburg/4/2009 [H1N1]) or laboratory-adapted FLUAV (A/PR/8/1934 [H1N1]), followed by quantification of viral release via focus formation assay. Notably, release of the laboratory-adapted virus was inhibited by tetherin but not ACE2 expression, and the inhibition efficiency correlated with the amount of tetherin expression (not shown), while similar effects were not observed for the pandemic virus. To extend these observations, we next compared the impacts of tetherin on release of a seasonal FLUAV (A/Panama/2007/1999 [H3N2]), laboratory-adapted FLUAV (A/PR/8/1934 [H1N1] and A/WSN/1933 [H1N1]), or pandemic FLUAV (A/Hamburg/4/2009 [H1N1]). Release of all seasonal and laboratory-adapted FLUAV was markedly inhibited by tetherin, while release of the pandemic virus was not affected (Fig. 3B), in keeping with our previous finding (Fig. 3A). Finally, analysis of MDCK cells, which are highly permissive to FLUAV replication, revealed that expression of tetherin reduced release of A/PR/8/1934 but not A/Hamburg/4/2009 (H1N1), despite comparable release from control transfected cells (Fig. 3C) (note that the reduced titers of $\mathrm{A} / \mathrm{PR} / 8 / 1934$ upon infection at an MOI of 1 compared to MOI of 0.1 were most likely due to cytopathic effects). These results suggest that sensitivity to inhibition by tetherin is strain dependent, with A/Hamburg/4/2009 (H1N1) and potentially other pandemic viruses being resistant to tetherin while several seasonal or laboratory-adapted viruses are tetherin sensitive.

NA and HA confer tetherin resistance to pandemic viruses. We next addressed the question whether the hemagglutinin (HA)and neuraminidase (NA)-encoding segments account for the relative tetherin insensitivity of the 2009 pandemic FLUAV. For this 
endeavor, we employed an $\mathrm{A} / \mathrm{PR} / 8 / 1934$ reassortant equipped with the HA and NA segments from pandemic A/Hamburg/4/ 2009. This virus, termed PR $8 \times 2009 \mathrm{HA} / \mathrm{NA}$, and the corresponding wild-type $(\mathrm{wt})$ viruses were tested for tetherin sensitivity, employing transfected 293T cells, as described above. Release of wt $\mathrm{A} / \mathrm{PR} / 8 / 1934$ was readily inhibited upon tetherin expression, while A/Hamburg/4/2009 release was not affected (Fig. 3D), confirming our previous observations. Importantly, the reassortant virus PR8 $\times 2009 \mathrm{HA} / \mathrm{NA}$ was resistant against tetherin's antiviral activity. Furthermore, a reassortant virus expressing the HA and NA segments of pandemic A/Aichi/2/1968 (H3N2) in the background of A/PR/8/1934 $(\mathrm{PR} 8 \times 1968 \mathrm{HA} / \mathrm{NA})$ turned out to be resistant against inhibition by tetherin (Fig. $3 \mathrm{E}$ ), indicating that the HA and NA proteins from several pandemic FLUAV can protect these viruses from inhibition by tetherin, at least in transfected 293T cells. In order to analyze whether HA and NA can also rescue FLUAV from inhibition by endogenous tetherin, we used siRNA knockdown to reduce tetherin levels in the lung-derived cell line NCI-H358 (Fig. 4A). The knockdown of tetherin expression markedly augmented release of A/PR/8/1934 but not A/Hamburg/4/2009 (Fig. 4B), and this effect was dependent on HA and NA, since release of PR8 reassortants bearing HA and NA of pandemic viruses was not modulated by tetherin knockdown. Thus, the HA and NA proteins of pandemic FLUAV can antagonize exogenous and endogenous tetherin.

Tetherin antagonism by HA and NA depends on the virion context and correlates with the ability to reduce tetherin expression. In order to obtain initial insights into determinants and mechanisms underlying tetherin antagonism by FLUAV, we next asked whether HA/NA are able to counteract inhibition of HIV budding by tetherin. This question was first addressed in a previously described VLP system (42), in which formation and release of VLPs are driven by HIV-1 p55 Gag. Release of Gag-VLPs was inhibited by tetherin, and this blockade was alleviated by expression of Vpu (Fig. 5A), as expected. In contrast, expression of 1918 $\mathrm{HA}$ and NA, either alone or in combination, did not rescue VLP release from inhibition by tetherin (Fig. $5 \mathrm{~A}$ ) (note that HA expression reduced VLP release due to binding to sialic acids [41]). Similarly, infection of Gag-expressing 293T cells with FLUAV (A/ Hamburg/4/2009, PR8 $\times 1968$ HA/NA) did not abrogate the tetherin-dependent blockade of VLP release (Fig. 5B). Thus, the ability of HA/NA to antagonize tetherin depends on the virion context.

Several tetherin antagonists reduce tetherin expression levels in infected cells. Therefore, we asked whether FLUAV resistant to tetherin interfere with tetherin expression levels. Tetherin expression in transfected 293T cells was not reduced upon infection with A/PR/8/1934, while a modest but statistically significant decrease in tetherin levels was observed in A/Hamburg/4/2009-infected cells (Fig. 6). Moreover, the introduction of the HA and NA segments from pandemic viruses into A/PR/8/1934 endowed the reassortants with the ability to reduce tetherin expression (Fig. 6). The efficiency of tetherin downregulation was dependent on the amount of tetherin plasmid transfected, and downregulation was also observed in NCI-H358 cells (not shown). These observations suggest that HA- and NA-dependent interference with tetherin expression might contribute to tetherin antagonism of FLUAV.
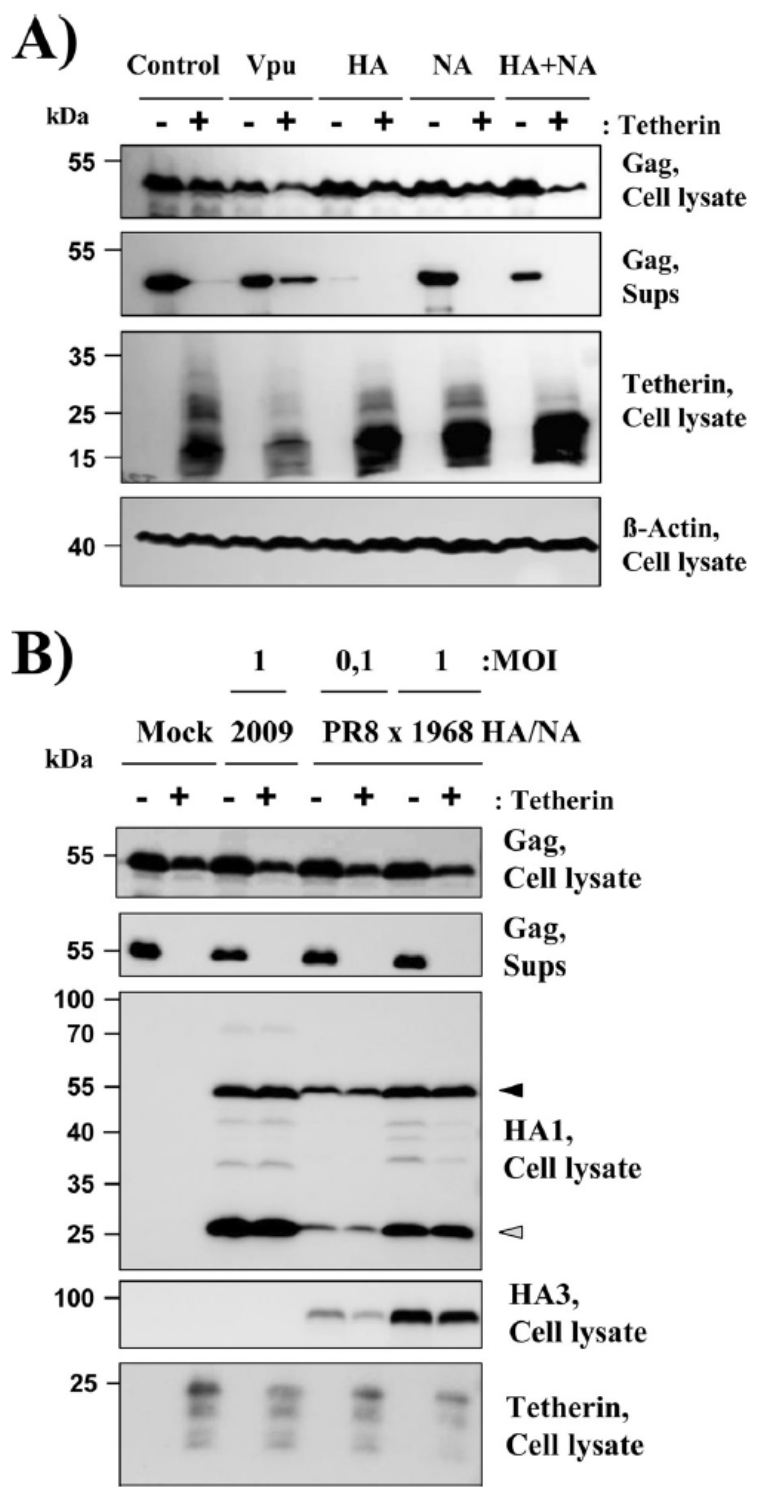

FIG 5 Tetherin antagonism by hemagglutinin and neuraminidase depends on the virion context. (A) $293 \mathrm{~T}$ cells were cotransfected with a plasmid encoding HIV-1 Gag and an empty plasmid or tetherin-encoding plasmid. In addition, cells were transfected with plasmids encoding Vpu, 1918 HA, or 1918 NA or encoding no protein (control). At $40 \mathrm{~h}$ posttransfection, the presence of Gag in culture supernatants and cell lysates was determined by Western blotting. In addition, the expression of tetherin and $\beta$-actin in cell lysates was determined. Similar results were obtained in three separate experiments. (B) 293T cells were cotransfected with a plasmid encoding HIV-1 Gag and an empty plasmid or tetherin-encoding plasmid and infected with the indicated FLUAV at the indicated MOIs. As a negative control, the cells were mock infected. At $24 \mathrm{~h}$ postinfection, supernatants (sups) were analyzed for expression of Gag, while cell lysates were analyzed for expression of Gag, tetherin, $\beta$-actin, and FLUAV proteins (anti-FLUAV H1N1 antibody was used for detection of viral proteins in $\mathrm{A} / \mathrm{Hamburg} / 4 / 2009$ [H1N1]-infected cells, while an HA-specific antibody was employed to probe lysates from PR $8 \times 1968 \mathrm{HA} / \mathrm{NA}$-infected cells). Similar results were obtained in at least three separate experiments. Bands with the molecular masses expected for NP and M1 proteins are marked with blackfilled (NP) and gray-filled (M1) triangles. MOI, multiplicity of infection; HA, hemagglutinin; NA, neuraminidase. 

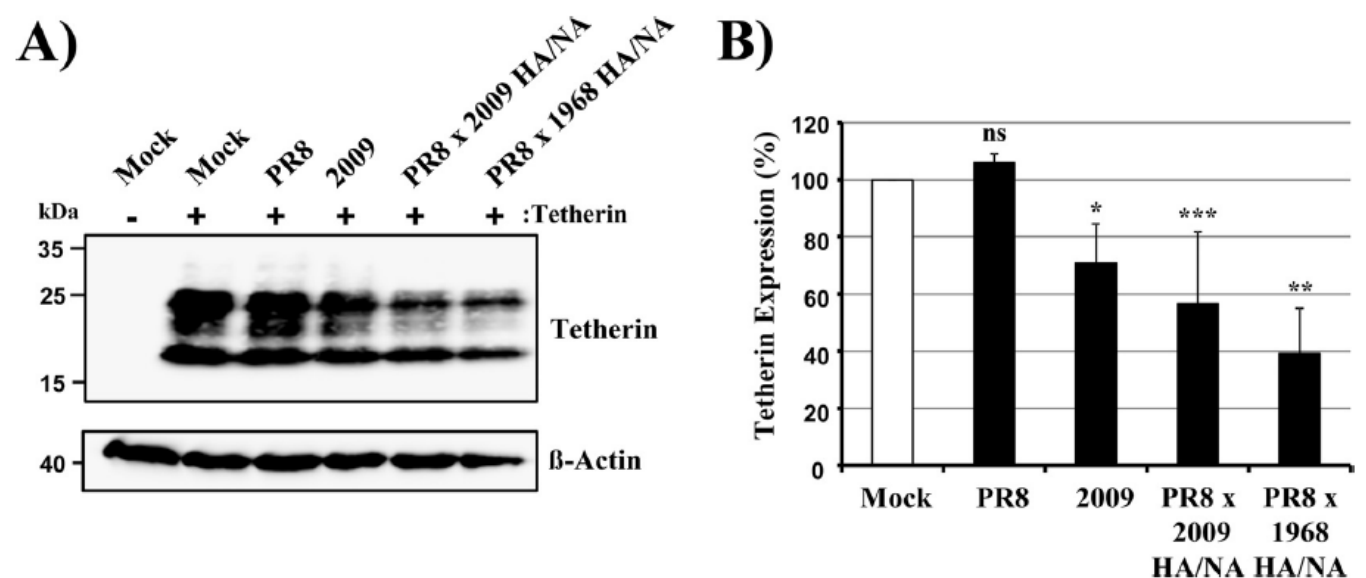

FIG 6 Neuraminidase and hemagglutinin of pandemic FLUAV reduce tetherin expression. (A) 293T cells were transfected with a tetherin-encoding plasmid or empty plasmid and infected with A/PR/8/1934 (H1N1) (abbreviated PR8), A/Hamburg/4/2009 (H1N1) (abbreviated 2009), and a reassortant PR8 virus equipped with HA and NA of 2009 (PR8 $\times 2009$ HA/NA) or A/Aichi/2/1968 (H3N2) $($ PR8 $\times 1968$ HA/NA) at an MOI of $0.1($ PR8, PR8 $\times 2009$ $\mathrm{HA} / \mathrm{NA}$, and PR $8 \times 1968 \mathrm{HA} / \mathrm{NA}$ ) or $0.3(2009)$. At $1 \mathrm{~h}$ postinfection, the virus-containing medium was replaced by fresh culture medium, and cells were cultured for $24 \mathrm{~h}$. Thereafter, cells were lysed and expression of tetherin and $\beta$-actin in cell lysates determined by Western blotting. Similar results were obtained in three separate experiments. (B) The tetherin and $\beta$-actin signals measured for panel A were quantified using ImageJ software and relative tetherin expression determined. Results represent the average from three independent experiments. Tetherin levels in mock-infected cells were set as $100 \%$. The statistical significance of differences between tetherin expression in mock-infected and FLUAV-infected cells was assessed by Student's $t$ test.

\section{DISCUSSION}

Tetherin can restrict the release of a variety of enveloped VLPs, including retro-, filo-, and arenavirus-like particles, by forming a clamp between the infected cell and the budding virion $(7,43)$. However, several but not all of the corresponding authentic viruses encode tetherin antagonists, which allow for efficient viral spread in tetherin-positive target cells $(7,43)$. Elucidating how these proteins inhibit tetherin may reveal novel targets for antiviral intervention and is thus in the spotlight of current research efforts. The present study shows that several seasonal or laboratory-adapted FLUAV are inhibited by tetherin, while several pandemic viruses antagonize tetherin via their HA and NA proteins. Moreover, our work demonstrates that tetherin antagonism by the HA and NA proteins is dependent on the virion context and might involve interference with tetherin expression.

Studies employing FLUAV VLP systems provided evidence that release of FLUAV-like particles can be restricted by tetherin $(18,23)$, although some viral NAs counteract tetherin with modest efficiency $(20,21,23)$, potentially by altering tetherin glycosylation (21). However, these studies did not examine HA and NA jointly for tetherin antagonism and/or did not analyze HA and NA proteins from pandemic FLUAV. Our results show that both 1918 NA and WSN NA can drive VLP budding, although with differential efficiency, and that VLP release is inhibited by tetherin. Notably, coexpression of 1918 HA rescued release of 1918 NAbased VLPs from inhibition by tetherin, while the same effect was not observed for WSN NA and HA. In contrast, 1918 HA failed to consistently antagonize tetherin in the context of WSN NA-driven VLP release. These results demonstrate that 1918 HA can antagonize tetherin in the presence of its cognate NA and suggest that WSN HA may not be able to do so, although the interpretation of the latter finding is complicated by the less robust expression of WSN HA compared to $1918 \mathrm{HA}$.

WSN was derived from a seasonal FLUAV, which was passaged in animals, while the FLUAV of the year 1918 is a pandemic virus.
The differential ability of the HA proteins of these viruses to rescue NA-driven VLP release from inhibition by tetherin suggested that seasonal or laboratory-adapted and pandemic FLUAV might differ in their susceptibilities to inhibition by tetherin. Indeed, all seasonal and laboratory-adapted FLUAV tested were inhibited by tetherin, while the pandemic virus A/Hamburg/4/2009 was resistant. Furthermore, the analysis of reassortant viruses showed that the introduction of the HA and NA segments of a pandemic virus into the PR8 background was sufficient to confer tetherin resistance to an otherwise susceptible virus. This finding is not in conflict with most previous studies documenting FLUAV inhibition by tetherin, which exclusively examined seasonal or laboratoryadapted FLUAV $(17-19,21)$, and parallels the documented observation that the surface proteins of Sendai virus, F and HN, are both required for tetherin antagonism (24). Dittmann and colleagues recently showed comparable inhibition of pandemic 2009 virus by tetherin and the antiviral effector IFITM3. However, the inhibition efficiency was low, while blockade of a swine FLUAV assayed in parallel was robust (22). Collectively, the results obtained with VLPs and authentic FLUAV indicate that certain pandemic FLUAV are resistant to tetherin and that tetherin resistance is determined by HA and NA proteins. In this context, it is noteworthy that recent studies documented an HA-dependent resistance of pandemic FLUAV to innate immune proteins of the collectin family, suggesting that the surface proteins of pandemic viruses might be better adapted to resist attack by effectors of the innate immune system than their counterparts in seasonal viruses $(44,45)$.

The finding that release of seasonal and laboratory-adapted FLUAV can be inhibited about 10-fold in the 293T cell system employed in the present study is of note, given that two studies reported similar effects $(20,46)$ while three independent ones found that inhibition of FLUAV release by tetherin is minor (2- to 5 -fold) or absent (17-19). Differences in the cellular systems, in particular the efficiency of tetherin expression, most likely ac- 
count for these discrepant findings. Therefore, future studies should determine which copy numbers of tetherin at the cell surface are required for inhibition of FLUAV release and whether the inhibitory activity of tetherin is to some degree dependent on the cellular context.

Many viral tetherin antagonists remove tetherin from the site of viral budding, the plasma membrane. For instance, the HIV-1 $\mathrm{Vpu}$ protein degrades tetherin and additionally interferes with tetherin transport to the cell surface $(6,7,12-14)$. In contrast, the Ebola virus glycoprotein counteracts tetherin without interfering with tetherin expression or cellular localization $(27,42,47-49)$, and it is currently unclear how this protein interferes with tetherin's antiviral activity. The viruses encoding HA and NA proteins capable of counteracting tetherin also reduced tetherin expression, at least when small amounts of plasmid were transfected, and it is conceivable that the two processes are linked. However, it will be challenging to investigate this possibility, since blockade of proteasomal and endosomal/lysosomal degradation of proteins will also interfere with FLUAV entry into host cells, which is known to depend on endosomal low $\mathrm{pH}$ and activity of proteasomes (50, 51). Finally, it is noteworthy that infection with A/Hamburg/4/ 2009, A/PR/8/1934, and PR $8 \times 2009 \mathrm{HA} / \mathrm{NA}$ did not interfere with tetherin expression at the cell surface, and only a modest reduction was observed upon PR $8 \times 1968 \mathrm{HA} / \mathrm{NA}$ infection in some experiments (data not shown). Lack of interference with tetherin surface expression might thus account for the observation that HA and NA expression or FLUAV infection did not rescue retroviral VLPs from inhibition by tetherin (an additional explanation is discussed below).

An alternative and mutually nonexclusive explanation for the strain dependence of tetherin antagonism might come from differences in the viral envelope: it is conceivable that the dense packaging of HA and NA in the viral membrane sterically impedes the anchoring of tetherin in the lipid bilayer. As a consequence, isolate-specific differences in the efficiency of virion incorporation of $\mathrm{HA}$ and NA, which have been documented (52), may result in differential tetherin sensitivity. It may thus be worthwhile to determine whether tetherin-resistant and -susceptible viruses differ in the amounts of HA and NA copies incorporated into viral particles and whether such differences impact susceptibility to inhibition by tetherin. Finally, it is noteworthy that Vpu rescues HIVand FLUAV-derived VLPs from inhibition by tetherin, while the tetherin-antagonizing activity of HA/NA is FLUAV specific. The dependence of HA/NA-mediated tetherin antagonism on the virion context is not unprecedented, since the Ebola virus glycoprotein rescues release of retro- and filoviral but not arenaviral VLPs from tetherin-positive cells (53). In fact, the context dependence of tetherin antagonism further underlines that efficient virion incorporation of HA and NA might contribute to tetherin inhibition. Thus, HIV-like particles use different compartments of the plasma membrane for budding relative to FLUAV-like particles (54) and might thus incorporate fewer HA/NA copies than their FLUAV counterparts.

Collectively, the present study provides evidence that pandemic FLUAV counteract tetherin via their HA/NA proteins, which might promote viral spread in the host, given that tetherin is constitutively expressed in the human lung (55) and is inducibly expressed upon IFN treatment (7). Future studies need to determine the underlying mechanism and might uncover novel targets for antiviral intervention. Furthermore, it will be interesting to examine if seasonal FLUAV are generally tetherin sensitive, which is at first sight counterintuitive but might be the result of adaptation of HA to immune pressure.

\section{ACKNOWLEDGMENTS}

We thank C. Basler for advice and the kind gift of the PR $8 \times 1968 \mathrm{HA} / \mathrm{NA}$ virus and A. Huwiler for NCI-H358 cells.

We thank the Leibniz Foundation (Graduate School Emerging Infectious Diseases [EIDIS]), DFG (PO 716/6-1, PO 716/8-1, SCHI 1073/4-1), the German Federal Ministry of Health (IIA5-2510.FSB-702 to T.W.), and HBRS/Center for Infection Biology/DEWIN (to K.G.) for support. This work was partly supported by the Göttingen Graduate School for Neurosciences, Biophysics, and Molecular Biosciences (DFG grant GSC 226/1 and DFG grant GSC 226/2).

\section{REFERENCES}

1. Garcia-Sastre A, Biron CA. 2006. Type 1 interferons and the virus-host relationship: a lesson in detente. Science 312:879-882. http://dx.doi.org /10.1126/science.1125676.

2. Ivashkiv LB, Donlin LT. 2014. Regulation of type I interferon responses. Nat Rev Immunol 14:36-49. http://dx.doi.org/10.1038/nri3581.

3. MacMicking JD. 2012. Interferon-inducible effector mechanisms in cellautonomous immunity. Nat Rev Immunol 12:367-382. http://dx.doi.org $/ 10.1038 /$ nri3210.

4. Yan N, Chen ZJ. 2012. Intrinsic antiviral immunity. Nat Immunol 13: 214-222. http://dx.doi.org/10.1038/ni.2229.

5. Neil SJ, Zang T, Bieniasz PD. 2008. Tetherin inhibits retrovirus release and is antagonized by HIV-1 Vpu. Nature 451:425-430. http://dx.doi.org /10.1038/nature06553.

6. Van Damme N, Goff D, Katsura C, Jorgenson RL, Mitchell R, Johnson MC, Stephens EB, Guatelli J. 2008. The interferon-induced protein BST-2 restricts HIV-1 release and is downregulated from the cell surface by the viral Vpu protein. Cell Host Microbe 3:245-252. http://dx.doi.org /10.1016/i.chom.2008.03.001.

7. Neil SJ. 2013. The antiviral activities of tetherin. Curr Top Microbiol Immunol 371:67-104. http://dx.doi.org/10.1007/978-3-642-37765-5_3.

8. Dave VP, Hajjar F, Dieng MM, Haddad E, Cohen EA. 2013. Efficient BST2 antagonism by Vpu is critical for early HIV-1 dissemination in humanized mice. Retrovirology 10:128. http://dx.doi.org/10.1186 /1742-4690-10-128.

9. Li SX, Barrett BS, Heilman KJ, Messer RJ, Liberatore RA, Bieniasz PD, Kassiotis G, Hasenkrug KJ, Santiago ML. 2014. Tetherin promotes the innate and adaptive cell-mediated immune response against retrovirus infection in vivo. J Immunol 193:306-316. http://dx.doi.org /10.4049/jimmunol.1400490.

10. Perez-Caballero D, Zang T, Ebrahimi A, McNatt MW, Gregory DA, Johnson MC, Bieniasz PD. 2009. Tetherin inhibits HIV-1 release by directly tethering virions to cells. Cell 139:499-511. http://dx.doi.org/10 .1016/i.cell.2009.08.039.

11. Venkatesh S, Bieniasz PD. 2013. Mechanism of HIV-1 virion entrapment by tetherin. PLoS Pathog 9:e1003483. http://dx.doi.org/10.1371/journal .ppat.1003483.

12. Andrew AJ, Miyagi E, Strebel K. 2011. Differential effects of human immunodeficiency virus type $1 \mathrm{Vpu}$ on the stability of BST-2/tetherin. J Virol 85:2611-2619. http://dx.doi.org/10.1128/JVI.02080-10.

13. Douglas JL, Viswanathan K, McCarroll MN, Gustin JK, Fruh K, Moses AV. 2009. Vpu directs the degradation of the human immunodeficiency virus restriction factor BST-2/Tetherin via a $\beta \mathrm{TrCP}$-dependent mechanism. J Virol 83:7931-7947. http://dx.doi.org/10.1128/JVI.00242-09.

14. Mitchell RS, Katsura C, Skasko MA, Fitzpatrick K, Lau D, Ruiz A, Stephens EB, Margottin-Goguet F, Benarous R, Guatelli JC. 2009. Vpu antagonizes BST-2-mediated restriction of HIV-1 release via beta-TrCP and endo-lysosomal trafficking. PLoS Pathog 5:e1000450. http://dx.doi .org/10.1371/journal.ppat.1000450.

15. Molinari NA, Ortega-Sanchez IR, Messonnier ML, Thompson WW, Wortley PM, Weintraub E, Bridges CB. 2007. The annual impact of seasonal influenza in the US: measuring disease burden and costs. Vaccine 25:5086-5096. http://dx.doi.org/10.1016/j.vaccine.2007.03.046.

16. Rossman JS, Lamb RA. 2011. Influenza virus assembly and budding. Virology 411:229-236. http://dx.doi.org/10.1016/j.virol.2010.12.003. 
17. Bruce EA, Abbink TE, Wise HM, Rollason R, Galao RP, Banting G, Neil SJ, Digard P. 2012. Release of filamentous and spherical influenza A virus is not restricted by tetherin. J Gen Virol 93:963-969. http://dx.doi.org/10 .1099/vir.0.038778-0.

18. Watanabe R, Leser GP, Lamb RA. 2011. Influenza virus is not restricted by tetherin whereas influenza VLP production is restricted by tetherin. Virology 417:50-56. http://dx.doi.org/10.1016/j.virol.2011.05.006.

19. Winkler M, Bertram S, Gnirss K, Nehlmeier I, Gawanbacht A, Kirchhoff F, Ehrhardt C, Ludwig S, Kiene M, Moldenhauer AS, Goedecke U, Karsten CB, Kuhl A, Pöhlmann S. 2012. Influenza A virus does not encode a tetherin antagonist with Vpu-like activity and induces IFNdependent tetherin expression in infected cells. PLoS One 7:e43337. http: //dx.doi.org/10.1371/journal.pone.0043337.

20. Leyva-Grado VH, Hai R, Fernandes F, Belicha-Villanueva A, Carter C, Yondola MA. 2014. Modulation of an ectodomain motif in the influenza A virus neuraminidase alters tetherin sensitivity and results in virus attenuation in vivo. J Mol Biol 426:1308-1321. http://dx.doi.org/10.1016/j.jmb .2013 .12 .023 .

21. Mangeat B, Cavagliotti L, Lehmann M, Gers-Huber G, Kaur I, Thomas Y, Kaiser L, Piguet V. 2012. Influenza virus partially counteracts restriction imposed by tetherin/BST-2. J Biol Chem 287:22015-22029. http://dx .doi.org/10.1074/jbc.M111.319996.

22. Dittmann M, Hoffmann HH, Scull MA, Gilmore RH, Bell KL, Ciancanelli M, Wilson SJ, Crotta S, Yu Y, Flatley B, Xiao JW, Casanova JL, Wack A, Bieniasz PD, Rice CM. 2015. A serpin shapes the extracellular environment to prevent influenza A virus maturation. Cell 160:631-643. http://dx.doi.org/10.1016/j.cell.2015.01.040.

23. Yondola MA, Fernandes F, Belicha-Villanueva A, Uccelini M, Gao Q, Carter C, Palese P. 2011. Budding capability of the influenza virus neuraminidase can be modulated by tetherin. J Virol 85:2480-2491. http://dx .doi.org/10.1128/JVI.02188-10.

24. Bampi C, Rasga L, Roux L. 2013. Antagonism to human BST-2/tetherin by Sendai virus glycoproteins. J Gen Virol 94:1211-1219. http://dx.doi.org /10.1099/vir.0.051771-0.

25. Blondeau C, Pelchen-Matthews A, Mlcochova P, Marsh M, Milne RS, Towers GJ. 2013. Tetherin restricts herpes simplex virus 1 and is antagonized by glycoprotein M. J Virol 87:13124-13133. http://dx.doi.org/10 $.1128 /$ JVI.02250-13.

26. Gupta RK, Mlcochova P, Pelchen-Matthews A, Petit SJ, Mattiuzzo G, Pillay D, Takeuchi Y, Marsh M, Towers GJ. 2009. Simian immunodeficiency virus envelope glycoprotein counteracts tetherin/BST-2/CD317 by intracellular sequestration. Proc Natl Acad Sci U S A 106:20889-20894. http://dx.doi.org/10.1073/pnas.0907075106.

27. Kaletsky RL, Francica JR, Agrawal-Gamse C, Bates P. 2009. Tetherinmediated restriction of filovirus budding is antagonized by the Ebola glycoprotein. Proc Natl Acad Sci U S A 106:2886-2891. http://dx.doi.org/10 $.1073 /$ pnas.0811014106.

28. Le Tortorec A, Neil SJ. 2009. Antagonism to and intracellular sequestration of human tetherin by the human immunodeficiency virus type 2 envelope glycoprotein. J Virol 83:11966-11978. http://dx.doi.org/10.1128 /JVI.01515-09.

29. Yin X, Hu Z, Gu Q, Wu X, Zheng YH, Wei P, Wang X. 2014. Equine tetherin blocks retrovirus release and its activity is antagonized by equine infectious anemia virus envelope protein. J Virol 88:1259-1270. http://dx .doi.org/10.1128/JVI.03148-13.

30. Brower M, Carney DN, Oie HK, Gazdar AF, Minna JD. 1986. Growth of cell lines and clinical specimens of human non-small cell lung cancer in a serum-free defined medium. Cancer Res 46:798-806.

31. Nguyen KL, M Ilano, Akari H, Miyagi E, Poeschla EM, Strebel K, Bour S. 2004. Codon optimization of the HIV-1 vpu and vif genes stabilizes their mRNA and allows for highly efficient Rev-independent expression. Virology 319:163-175. http://dx.doi.org/10.1016/j.virol.2003.11.021.

32. Sauter D, Schindler M, Specht A, Landford WN, Munch J, Kim KA, Votteler J, Schubert U, Bibollet-Ruche F, Keele BF, Takehisa J, Ogando Y, Ochsenbauer C, Kappes JC, Ayouba A, Peeters M, Learn GH, Shaw G, Sharp PM, Bieniasz P, Hahn BH, Hatziioannou T, Kirchhoff F. 2009. Tetherin-driven adaptation of Vpu and Nef function and the evolution of pandemic and nonpandemic HIV-1 strains. Cell Host Microbe 6:409421. http://dx.doi.org/10.1016/j.chom.2009.10.004.

33. Chaipan C, Kobasa D, Bertram S, Glowacka I, Steffen I, Tsegaye TS, Takeda M, Bugge TH, Kim S, Park Y, Marzi A, Pöhlmann S. 2009. Proteolytic activation of the 1918 influenza virus hemagglutinin. J Virol 83:3200-3211. http://dx.doi.org/10.1128/JVI.02205-08.
34. Gao F, Li Y, Decker JM, Peyerl FW, Bibollet-Ruche F, Rodenburg CM, Chen Y, Shaw DR, Allen S, Musonda R, Shaw GM, Zajac AJ, Letvin N, Hahn BH. 2003. Codon usage optimization of HIV type 1 subtype $\mathrm{C}$ gag, pol, env, and nef genes: in vitro expression and immune responses in DNA-vaccinated mice. AIDS Res Hum Retroviruses 19:817-823. http: //dx.doi.org/10.1089/088922203769232610.

35. Schneider CA, Rasband WS, Eliceiri KW. 2012. NIH Image to ImageJ: 25 years of image analysis. Nat Methods 9:671-675. http://dx.doi.org/10 $.1038 /$ nmeth. 2089 .

36. Abt M, de jonge J, Laue M, Wolff T. 2011. Improvement of H5N1 influenza vaccine viruses: influence of internal gene segments of avian and human origin on production and hemagglutinin content. Vaccine 29: 5153-5162. http://dx.doi.org/10.1016/j.vaccine.2011.05.036.

37. Baez M, Palese P, Kilbourne ED. 1980. Gene composition of highyielding influenza vaccine strains obtained by recombination. J Infect Dis 141:362-365. http://dx.doi.org/10.1093/infdis/141.3.362.

38. Terrier O, Moules V, Carron C, Cartet G, Frobert E, Yver M, Traversier A, Wolff T, Riteau B, Naffakh N, Lina B, Diaz J, Rosa-Calatrava M. 2012. The influenza fingerprints: NS1 and M1 proteins contribute to specific host cell ultrastructure signatures upon infection by different influenza A viruses. Virology 432:204-218. http://dx.doi.org/10.1016/j.virol .2012.05.019.

39. Wrensch F, Winkler M, Pöhlmann S. 2014. IFITM proteins inhibit entry driven by the MERS-coronavirus spike protein: evidence for cholesterolindependent mechanisms. Viruses 6:3683-3698. http://dx.doi.org/10 .3390/v6093683.

40. Hatesuer B, Bertram S, Mehnert N, Bahgat MM, Nelson PS, Pohlman S, Schughart K. 2013. Tmprss2 is essential for influenza H1N1 virus pathogenesis in mice. PLoS Pathog 9:e1003774. http://dx.doi.org/10.1371 /journal.ppat.1003774.

41. Bosch V, Kramer B, Pfeiffer T, Starck L, Steinhauer DA. 2001. Inhibition of release of lentivirus particles with incorporated human influenza virus haemagglutinin by binding to sialic acid-containing cellular receptors. J Gen Virol 82:2485-2494.

42. Kühl A, Banning C, Marzi A, Votteler J, Steffen I, Bertram S, Glowacka I, Konrad A, Sturzl M, Guo JT, Schubert U, Feldmann H, Behrens G, Schindler M, Pöhlmann S. 2011. The Ebola virus glycoprotein and HIV-1 Vpu employ different strategies to counteract the antiviral factor tetherin. J Infect Dis 204(Suppl 3):S850-S860. http://dx.doi.org/10.1093/infdis/jir378.

43. Sauter D. 2014. Counteraction of the multifunctional restriction factor teth erin. Front Microbiol 5:163. http://dx.doi.org/10.3389/fmicb.2014.00163.

44. Job ER, Deng YM, Tate MD, Bottazzi B, Crouch EC, Dean MM, Mantovani A, Brooks AG, Reading PC. 2010. Pandemic H1N1 influenza A viruses are resistant to the antiviral activities of innate immune proteins of the collectin and pentraxin superfamilies. J Immunol 185:4284-4291. http://dx.doi.org/10.4049/jimmunol.1001613.

45. Qi L, Kash JC, Dugan VG, Jagger BW, Lau YF, Sheng ZM, Crouch EC, Hartshorn KL, Taubenberger JK. 2011. The ability of pandemic influenza virus hemagglutinins to induce lower respiratory pathology is associated with decreased surfactant protein D binding. Virology 412:426-434. http: //dx.doi.org/10.1016/j.virol.2011.01.029.

46. Mangeat B, Gers-Huber G, Lehmann M, Zufferey M, Luban J, Piguet V. 2009. HIV-1 Vpu neutralizes the antiviral factor tetherin/BST-2 by binding it and directing its beta-TrCP2-dependent degradation. PLoS Pathog 5:e1000574. http://dx.doi.org/10.1371/journal.ppat.1000574.

47. Gnirss K, Fiedler M, Kramer-Kuhl A, Bolduan S, Mittler E, Becker S, Schindler M, Pöhlmann S. 2014. Analysis of determinants in filovirus glycoproteins required for tetherin antagonism. Viruses 6:1654-1671. http://dx.doi.org/10.3390/v6041654.

48. Lopez LA, Yang SJ, Hauser H, Exline CM, Haworth KG, Oldenburg J, Cannon PM. 2010. Ebola virus glycoprotein counteracts BST-2/tetherin restriction in a sequence-independent manner that does not require tetherin surface removal. J Virol 84:7243-7255. http://dx.doi.org/10.1128/JVI .02636-09.

49. Lopez LA, Yang SJ, Exline CM, Rengarajan S, Haworth KG, Cannon PM. 2012. Anti-tetherin activities of HIV-1 Vpu and Ebola virus glycoprotein do not involve removal of tetherin from lipid rafts. J Virol 86: 5467-5480. http://dx.doi.org/10.1128/JVI.06280-11.

50. Khor R, McElroy LJ, Whittaker GR. 2003. The ubiquitin-vacuolar protein sorting system is selectively required during entry of influenza virus into host cells. Traffic 4:857-868. http://dx.doi.org/10.1046/j.1398-9219 .2003.0140.x.

51. Widjaja I, de Vries E, Tscherne DM, Garcia-Sastre A, Rottier PJ, de 
Haan CA. 2010. Inhibition of the ubiquitin-proteasome system affects influenza A virus infection at a postfusion step. J Virol 84:9625-9631. http: //dx.doi.org/10.1128/JVI.01048-10.

52. Moules V, Terrier O, Yver M, Riteau B, Moriscot C, Ferraris O, Julien T, Giudice E, Rolland JP, Erny A, Bouscambert-Duchamp M, Frobert E, Rosa-Calatrava M, Pu LY, Hay A, Thomas D, Schoehn G, Lina B. 2011. Importance of viral genomic composition in modulating glycoprotein content on the surface of influenza virus particles. Virology 414:51-62. http://dx.doi.org/10.1016/j.virol.2011.03.011

53. Radoshitzky SR, Dong L, Chi X, Clester JC, Retterer C, Spurgers K, Kuhn JH, Sandwick S, Ruthel G, Kota K, Boltz D, Warren T, Kranzusch PJ, Whelan SP, Bavari S. 2010. Infectious Lassa virus, but not filoviruses, is restricted by BST-2/tetherin. J Virol 84:10569-10580. http://dx.doi.org /10.1128/JVI.00103-10.

54. Khurana S, Krementsov DN, de Vries E, Elder JH, Foti M, Thali M. 2007. Human immunodeficiency virus type 1 and influenza virus exit via different membrane microdomains. J Virol 81:12630-12640. http://dx .doi.org/10.1128/JVI.01255-07.

55. Erikson E, Adam T, Schmidt S, Lehmann-Koch J, Over B, Goffinet C, Harter C, Bekeredjian-Ding I, Sertel S, Lasitschka F, Keppler OT. 2011. In vivo expression profile of the antiviral restriction factor and tumor-targeting antigen CD317/BST-2/HM1.24/tetherin in humans. Proc Natl Acad Sci U S A 108:13688-13693. http://dx.doi.org/10.1073 /pnas.1101684108. 


\section{Fifth manuscript}

\section{Influenza virus activating host cell proteases antagonize tetherin}

Krämer-Kühl, A.*, Zmora, P.*, Blazejewska, P.*, Gnirß, K.*, Nehls, J., Bertram, S., Dijkman, R., Heurich, A., Karsten, C.B., Winkler, M., Wolff, T., Thiel, V., Schindler, M., Pöhlmann, S.

Journal of Virology, submitted on 12.10.2015 (submission number JVI02620-15)

Individual contribution:

Analysis of tetherin expression in the presence of TTSPs via Western blotting (Fig. 2A). Determination whether tetherin-mediated inhibition can be counteracted by TTSPs (Fig. 3A). Analysis of the effect of tetherin on the virus release (Fig. 6B). Analysis of the siRNA knockdown effect on the virus release (Fig. 6E). Writing parts of the manuscript (Material and Methods, Results) 


\title{
Influenza virus activating host cell proteases antagonize tetherin
}

\author{
Annika Krämer-Kühl, ${ }^{\text {a, } \dagger}$ Pawel Zmora, ${ }^{a}$ Paulina Blazejewska, ${ }^{\text {a, }}$ Kerstin Gnirß, a, ${ }^{\dagger}$ \\ Julia Nehls, ${ }^{\text {b,c }}$ Stephanie Bertram, ${ }^{\text {a, } \dagger}$ Ronald Dijkman, ${ }^{\text {d }}$ Adeline Heurich, ${ }^{a}$ \\ Christina B. Karsten, ${ }^{\mathrm{a}, \mathrm{e},{ }^{\dagger}}$ Michael Winkler, ${ }^{\mathrm{a}}$ Thorsten Wolff, ${ }^{\mathrm{f}}$ Volker Thiel, ${ }^{\mathrm{d}}$ \\ Michael Schindler, ${ }^{\text {b,c }}$ Stefan Pöhlmann, a,"
}

Infection Biology Unit, German Primate Center, Göttingen, Germany ${ }^{\mathrm{a}}$; Institute of Virology, Helmholtz Zentrum Munich, German Research Center for Environmental Health, Munich, Germany ${ }^{\mathrm{b}}$; Institute of Medical Virology and Epidemiology of Viral Diseases, University Clinic Tübingen, Tübingen, Germany ${ }^{c}$; Institute of Immunobiology, Kantonsspital St. Gallen, St.Gallen, Switzerland ${ }^{\text {; }}$ Institute for Cellular Chemistry, Hannover Medical School, Hannover, Germany ${ }^{\mathrm{e}}$; Division of Influenza Viruses and other Respiratory Viruses, Robert Koch-Institute, Berlin, Germany ${ }^{\mathrm{f}}$.

A.K.K., P.Z., P.B. and K.G. contributed equally to this work

${ }^{\dagger}$ Present address: Boehringer Ingelheim Veterinary Research Center GmbH \& Co. KG, Hannover, Germany (A.K.-K., P.B.); Heinrich-Pette-Institute, Leibniz Institute for Experimental Virology, Hamburg, Germany (S.B., K.G.); Ragon Institute of MGH, MIT and Harvard, Cambridge, MA, USA (C.B.K.).

*Corresponding author. Please direct correspondence to: Infection Biology Unit, German Primate Center, Kellnerweg 4, 37077 Göttingen, Germany. Phone: +49 5513851 150, Fax: +49 5513851 184, E-mail: spoehlmann@dpz.eu

Running title: Tetherin antagonism by host cell proteases 


\section{ABSTRACT}

The host cell protein tetherin can inhibit the release of enveloped viruses from infected cells and constitutes an important defense against viral infection. Influenza viruses, major human pathogens, are largely resistant against tetherin's antiviral activity but the mechanism underlying resistance is unclear. Here, we show that the cellular protease TMPRSS2, which activates the influenza virus hemagglutinin for host cell entry, also cleaves tetherin and facilitates viral release from tetherin-positive cells. TMPRSS2 was coexpressed with tetherin in bronchial epithelium, a target of influenza virus infection, suggesting that TMPRSS2 might promote viral release in the infected host. These results indicate that influenza viruses exploit TMPRSS2 to ensure two important functions in viral replication, hemagglutinin activation and viral release from infected cells. The unexpected finding that a virus-activating host cell factor can counter an innate antiviral defense mechanism may have important implications for understanding viral pathogenesis and development of antivirals. 


\section{IMPORTANCE}

Host cell encoded antiviral effector proteins can inhibit virus invasion and are important components of innate immune defenses. Understanding how viruses evade the antiviral activity of these effector proteins might afford novel approaches to antiviral therapy and is thus in the focus of current research efforts. It has been established that several viruses encode proteins, which antagonize antiviral effectors, for instance by reducing their expression. Here, we report for the first time that an antiviral effector, tetherin, can be inhibited by another host cell encoded protein, the protease TMPRSS2. These results suggest that the cellular environment can determine whether an antiviral effector protein can unfold its full activity and help to define TMPRSS2 as a therapeutic target. 


\section{INTRODUCTION}

Mammalian cells are equipped with antiviral effector proteins, which target specific components of invading viruses and restrict viral spread $(1,2)$. Expression of these virusrestricting factors (restriction factors) is frequently inducible by interferons (IFNs) and their activity is integral to the control of viral spread by the IFN system (1). As a consequence, viruses evolved countermeasures which antagonize restriction factors and allow efficient viral spread despite activation of the IFN system $(1,2)$. The host cell protein tetherin (BST-2, CD317) is an IFN-inducible restriction factor, which targets the viral envelope and inhibits release of progeny particles from infected cells (3). The antiviral activity of tetherin has initially been identified in the context of HIV-1 infection and the HIV-1 accessory protein Vpu was found to counteract tetherin $(4,5)$. Recent studies conducted in mice indicate that tetherin's potent anti-retroviral activity displayed in cell culture translates into robust inhibition of viral spread in the host (6-9), indicating that tetherin is an important component of the innate defenses against retroviral infection.

Tetherin exhibits a particular domain organization and membrane topology which is key to its antiviral activity $(3,10)$. It contains an $\mathrm{N}$-terminal cytoplasmic domain, followed by a membrane spanning domain, an extracellular coiled-coil domain and a C-terminal glycosylphosphatidylinositol (GPI)-anchor (11). The presence of two membrane anchors allows the protein to simultaneously insert into the viral and the host cell membrane. As a consequence, tetherin can form a physical tether between progeny virions and the plasma membrane of the host cell, which prevents release of infectious viral particles into the extracellular space $(4,12-15)$. The integral role of tetherin's topology in its antiviral action is underlined by the observation that an engineered protein, which does not share sequence homology with tetherin but exhibits a comparable domain organization, can also suppress viral release (12). 
The finding that tetherin targets the viral membrane and prevents release of virions from the plasma membrane suggests that most if not all enveloped viruses which exit host cells via the plasma membrane and do not encode a tetherin antagonist should be inhibited by tetherin. Indeed, evidence has been provided that - in the absence of viral countermeasures tetherin is active against many viruses belonging to diverse families, including retroviridae (4, 14-20), paramyxoviridae (21, 22), filoviridae (23-26), herpesviridae (27), flaviviridae (28, 29), togaviridae (30) and arenaviridae (22, 25, 31). Influenza viruses, members of the family orthomyxoviridae, constitute a notable exception: Although these viruses are released from the plasma membrane $(32,33)$, ambiguous results were reported concerning their sensitivity towards tetherin. Thus, three studies did not detect appreciable inhibition of influenza A virus (FLUAV) release by tetherin (34-36). The same number of reports indicated that release of FLUAV is blocked by tetherin but the inhibition efficiency was lower than that commonly observed for vpu-defective HIV-1 (37-39). Notably, tetherin inhibited release of FLUAV-like particles (fVLPs) but not authentic FLUAV $(35,40)$, suggesting that viral proteins lacking in fVLP-producing cells but present in FLUAV infected cells might function as tetherin antagonists. Indeed, variants of the viral neuraminidase (NA) with an aspartate at position 286 (N2)/268 (N1) were shown to be budding competent (40), to inhibit tetherin's antiviral activity in cell culture (38), potentially by modifying tetherin glycosylation (37), and to be associated with increased viral replication in mice (38), although the effects observed were generally modest. In addition, we recently reported that the HA proteins of pandemic but not seasonal or lab-adapted FLUAV can antagonize tetherin (41). Thus, FLUAV seems to encode tetherin antagonists but it is unlikely that they account for the full spectrum of tetherin resistance observed within previous studies.

Here, we addressed the possibility that host cell proteins present in FLUAV target cells might render the virus insensitive to inhibition by tetherin. We focused our study on host 
cell proteases, since activation of the FLUAV hemagglutinin (HA) by cellular proteases is essential for viral infectivity (42) and purified trypsin (which is commonly used to activate influenza viruses in protease-negative cells) or related proteases are known to abrogate tetherin's antiviral activity $(13,15,43)$. Specifically, we asked whether the type II transmembrane serine proteases (TTSPs) TMPRSS2, TMPRSS4 and HAT, DESC1 and MSPL, which activate HA in cell culture (44-46), also counteract tetherin. Particular focus was placed on TMPRSS2, since expression of this protease is essential for FLUAV spread in mice (47-49). We found that exogenous and endogenous expression of these proteins results in tetherin cleavage and inactivation of tetherin's antiviral function. Moreover, we demonstrate that TMPRSS2 and tetherin are coexpressed in the human lung. These observations indicate that FLUAV evades tetherin's antiviral activity by targeting cells which express tetherin-inactivating, HA-activating proteases. 


\section{MATERIAL AND METHODS}

Cell culture and plasmids. 293T cells were cultured in Dulbecco's modified Eagle medium (DMEM, Invitrogen) while Caco-2 cells were cultured in DMEM GlutaMax (Invitrogen). All media were supplemented with penicillin, streptomycin and $10 \%$ fetal bovine serum. Cells were culture in a humidified atmosphere containing $5 \% \mathrm{CO}_{2}$. The plasmids encoding HIV-1 NL4-3 wt and $\Delta v$ pu proviral DNA (50), proteases (46, 51-53), NA of the 1918 FLUAV (45), genomic segments of A/Panama/2007/99 (H3N2) (54), tetherin and viral tetherin antagonists (26) were described previously. Splice overlap extension PCR was employed to delete amino acids $21-48$ in tetherin, which comprise the complete transmembrane domain, resulting in construct tetherin $\triangle \mathrm{TM}$. The plasmids pECFP and pEYFP, pECFP-EYFP, pEYFP-Vpu and pECFP-MEM have already been described (55). TMPRSS2 and TMPRSS6 N-terminally fused to YFP were generated by PCR amplification of the protease coding sequence followed by insertion into plasmid pEYFP-C1 (Clontech). The integrity of all PCR-amplified sequences was verified by automated sequencing.

Analysis of tetherin expression. For analysis of tetherin expression in cell lysates, 293T cells coexpressing tetherin and viral tetherin antagonists or TTSPs were washed with PBS and lysed with SDS-loading buffer (50 mM Tris $\mathrm{pH} 6.8,10 \%$ glycerol, $2 \%$ SDS, 5\% $\beta$ mercaptoethanol, $0.1 \%$ bromphenolblue, 1 mM EDTA). Subsequently, samples were incubated for $30 \mathrm{~min}$ at $95^{\circ} \mathrm{C}$ and separated by SDS-PAGE. Proteins were transferred onto nitrocellulose membranes and detected employing rabbit anti-tetherin antisera ((56), Abcam) and horseradish-peroxidase-coupled anti-rabbit antibody (Dianova) or mouse monoclonal anti-tetherin antibody (eBIOSCIENCE) jointly with horseradish-peroxidase-coupled antimouse antibody (Dianova). Staining was visualized with ECL Prime Western Blotting 
Detection Reagent (Amersham) following the manufacturer's protocol. For analysis of tetherin expression at the cell surface, 293T cells coexpressing tetherin and viral tetherin antagonists or TTSPs were mechanically detached, washed with FACS buffer (PBS with $0.5 \%$ FCS) and stained with anti-tetherin monoclonal antibody (eBIOSCIENCE). Mouse

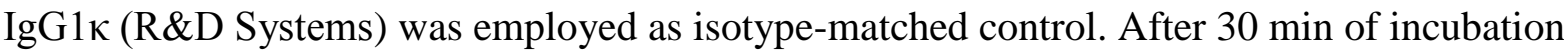
with primary antibodies at $4{ }^{\circ} \mathrm{C}$, cells were washed twice with FACS buffer and incubated for 30 min at $4^{\circ} \mathrm{C}$ with DyLight 647-coupled anti-mouse secondary antibodies (Dianova). After two final washing steps, cells were fixed with $2 \%$ paraformaldehyde and analyzed with a BD LSR II flow cytometer.

Analysis of tetherin antagonism employing lentiviral particles. To analyze inhibition of viral release by tetherin and its counteraction, a previously published VLP system was employed (26). In brief, 293T cells were seeded at a density of $2.6 \times 10^{5}$ cells/well in 6-well plates and calcium phosphate-cotransfected with plasmids encoding Gag, tetherin and either Vpu, EBOV-GP or TTSPs at a ratio of 2:1:1 (total amount of $6 \mu \mathrm{g}$ plasmid/well). As control, cells were cotransfected with empty plasmid. At $48 \mathrm{~h}$ after transfection, supernatants were collected and cleared by centrifugation at 3,500 g for $8 \mathrm{~min}$. Subsequently, supernatants were loaded on top of $20 \%$ sucrose in TNE buffer and centrifuged at 21,100 $\mathrm{g}$ for at least $2 \mathrm{~h}$ at $4^{\circ} \mathrm{C}$. Thereafter, supernatants were removed and concentrated VLPs were mixed with $30 \mu \mathrm{l}$ SDS-loading buffer. For production of cell lysates, the cells were washed with PBS and detached with $200 \mu \mathrm{l} /$ well SDS-loading buffer. For some experiments, concentrated VLPs and cell pellets were treated with PNGaseF for 1 hour at $37^{\circ} \mathrm{C}$ as described by the manufacturer's protocol (New England Biolabs). All samples were denatured for $30 \mathrm{~min}$ at $95^{\circ} \mathrm{C}$, separated by SDS-PAGE and proteins detected by Western blot, employing the anti- 
p24 hybridoma cell culture supernatant (183-H12-5C) for detection of Gag (57) and antiCD317 monoclonal antibody (eBIOSCIENCE) for detection of tetherin fragments.

siRNA knock-down and particle release from Caco-2 cells. Caco-2 cells were seeded in 6well plates at $3 \times 10^{5}$ cells/well. After $24 \mathrm{~h}$, the culture medium was replaced by Opti-MEM medium (Invitrogen) and the cells were cotransfected with 200 pmol scrambled siRNA or siRNAs specific for tetherin or TMPRSS2 (Santa Cruz) jointly with $4 \mu \mathrm{g}$ expression plasmids encoding for HIV-1 NL4-3 wt or HIV-1 NL4-3 $\Delta \mathrm{vpu}$, employing Lipofectamin 2000 as recommended by the manufacturer (Invitrogen). Alternatively, cells were cotransfected with siRNAs and influenza virus NA expression plasmid. Supernatants and cell lysates were harvested at $48 \mathrm{~h}$ post transfection and analyzed as described above for VLP-producing cells. Expression of NA was analyzed employing an anti-V5-specific antibody (Invitrogen).

Influenza A viruses. A/PR/8/34 (H1N1) and A/Panama/2007/99 (H3N2) were propagated in the chorio-allantoic cavity of 10-day-old embryonated hen eggs (Harlan Winkelman, Germany) for $48 \mathrm{~h}$ at $37^{\circ} \mathrm{C}$. A/Panama/2007/99 (H3N2) was reconstituted from an 8-plasmid system (54) before amplification in eggs.

Tetherin antagonism of influenza A virus release. 293T cells were seeded in 6-well plates at a density of $2.8 \times 10^{5}$ cells/well. After $24 \mathrm{~h}$, the cells were transfected with $6 \mu \mathrm{g}$ expression plasmids encoding for tetherin, TMPRSS2 or a catalytically inactive TMPRSS2 mutant using the calcium phosphate transfection method. The transfection medium was replaced by fresh medium after an overnight incubation. At $24 \mathrm{~h}$ post transfection the culture medium was removed and the cells were infected with $\mathrm{A} / \mathrm{PR} / 8 / 34(\mathrm{H} 1 \mathrm{~N} 1)$ at an MOI of 0.1 and with A/Panama/2007/99 (H3N2) at an MOI of 0.3. For this, culture medium was removed and 
virus diluted in PBS (containing $\mathrm{Ca}^{++}$and $\mathrm{Mg}^{++}$) supplemented with $0.2 \%$ BSA was added. After $1 \mathrm{~h}$, the infection medium was removed, the cells were gently washed and DMEM supplemented with $0.2 \%$ BSA was added. Culture supernatants were collected after either 8 or $24 \mathrm{~h}$ post infection. The amount of infectious units within the culture supernatants was determined by focus formation assay. Alternatively, Caco- 2 cells were seeded in 12 -well plates at a density of $1.4 \times 10^{5}$ cells/well and transfected with $1 \mu \mathrm{g}$ of siRNA specific for tetherin or TMPRSS2 or scrambled, non-sense siRNA as described above. At 24 h post transfection, the cells were infected with A/PR/8/34 (H1N1) at an MOI 0.01, as described above. The supernatant was collected at $24 \mathrm{~h}$ post infection, and the virus titers were determined using focus formation assay.

Focus formation assay. Virus titers were determined on MDCK II cells using the focus formation (FOCI) assay as described $(36,58)$. Briefly, MDCK II cells were seeded in 96-well culture plates at a density of 4-6 x $10^{4}$ cells/well and serial 2-, 5- or 10-fold dilutions of culture supernatants prepared in DMEM containing $2.5 \mu \mathrm{g} / \mathrm{ml}$ NAT (N-Acetylated Trypsin, Sigma), $0.1 \%$ BSA, $1 \%$ penicillin/streptomycin were added. After $1 \mathrm{~h}$ of incubation, inoculates were replaced with $100 \mu \mathrm{l}$ of $1 \%$ Avicel overlay medium and the cells were incubated for $24 \mathrm{~h}$. Subsequently, the cells were washed twice with PBS and fixed with 4\% formalin in PBS for $10 \mathrm{~min}$ at room temperature. Then, cells were washed twice and incubated with quencher $(100 \mu 1 /$ well; $0.5 \%$ Triton X-100, $20 \mathrm{mM}$ Glycine in PBS $)$ for $10 \mathrm{~min}$ at room temperature. Thereafter, cells were washed with washing buffer $(100 \mu 1 /$ well; $0.5 \%$ Tween20 in PBS) and blocked with blocking buffer (50 $\mu 1 /$ well; $0.5 \%$ Tween, $20 \%$ BSA in PBS) for $30 \mathrm{~min}$ at $37^{\circ} \mathrm{C}$ in $5 \% \mathrm{CO}_{2}$. Subsequently, primary antibody (anti-influenza NP polyclonal goat antibody, Virostat, or anti-FLUAV (H1N1), Millipore) was added and the cells incubated for $1 \mathrm{~h}$. Finally, the cells were washed and incubated with secondary antibody 
(anti-goat-HRP, Dianova) for $1 \mathrm{~h}$ in the dark, then washed again and incubated with substrate (True Blue from KPL, MA, USA) and exposed until blue spots from infected cells appeared. Foci were counted and viral titers were calculated as focus formation units (FFU) per ml of culture supernatant.

Confocal microscopy and FACS-based FRET assay. For the analysis of the subcellular localization of tetherin, TMPRSS2 and TMPRSS6, transfected 293T cells seeded on cover slips were calcium phosphate transfected to express the respective CFP and YFP-labeled fusion proteins. After 24 h, cover slips were mounted on objective slides with Mowiol containing DRAQ5 $(1 \mu \mathrm{g} / \mathrm{ml})$ to visualize DNA. Areas of fluorescent protein expression were visualized by confocal spinning disc microscopy at $63 \mathrm{x}$ magnification with an oil immersion objective (Nikon TiE equipped with the PerkinElmer UltraView Vox system). FACS-based FRET was performed and quantified essentially as described before $(55,59)$. Briefly, $293 \mathrm{~T}$ cells were seeded in 12-well plates at $1.5 \times 10^{5}$ cells/well and calcium phosphate transfected with $1.25 \mu \mathrm{g}$ of each CFP/YFP expression plasmid. At $6 \mathrm{~h}$ post transfection medium was changed and at $24 \mathrm{~h}$ post transfection cells were harvested for FACS-FRET analysis with a FACSCantoII (BD). CFP/YFP double positive cells were gated first and subsequently the FRET background signal was defined according to the pECFP and pEYFP cotransfected negative control. At least 3,000 double positive cells were analyzed for every transfection. For data evaluation, the percentage of cells exerting higher FRET fluorescence than the negative control was quantified. Filter settings were CFP (ex 405 / em 450), YFP (ex 488 / em 525) and FRET (ex 405 / em 525).

Immunohistochemistry. For in vivo examination of tetherin distribution in human airway epithelium, primary bronchi segments were fixed and immunostained as previously described 
(60). Mouse monoclonal anti-TMPRSS2 (Santa Cruz) and rabbit polyclonal anti-tetherin were applied as primary antibodies. Donkey derived, Dylight 488 labeled, anti-mouse IgG $(\mathrm{H}+\mathrm{L})$ and Dylight 647 labeled anti-rabbit $\operatorname{IgG}(\mathrm{H}+\mathrm{L})$ were applied as secondary antibodies. Thereafter, the cells were incubated with Cy3-conjugated mouse anti- $\beta$-tubulin (Tub2.1; Sigma Aldrich) for staining of ciliated cells and counterstained with DAPI (Invitrogen). Fluorescent images were acquired using EC Plan Neo-fluar 40x/1.30 Oil DIC M27 objective on a Zeiss LSM 710 confocal microscope. Image capture, analysis and processing were performed using the ZEN 2010 (Zeiss). 


\section{RESULTS}

FLUAV activating type II transmembrane serine proteases antagonize tetherin. We first investigated whether coexpression of TTSPs interferes with tetherin-mediated restriction of viral release. For this, we employed a well-established HIV-1 Gag-based VLP system or authentic HIV-1, since this experimental approach allows to measure anti-tetherin activity of TTSPs in the absence of confounding effects resulting from virus activation by these proteases (TTSPs activate FLUAV but not HIV-1). Coexpression of TTSPs did not impact expression of HIV-1 Gag in cell lysates or release of Gag from tetherin-negative cells (Fig. 1A). Similarly, no effect of Vpu and the Ebola virus glycoprotein (EBOV-GP), a tetherin antagonist (24), on Gag expression and release from tetherin-negative cells was seen. Release of Gag was markedly diminished upon coexpression of tetherin and this effect was largely rescued upon coexpression of Vpu or EBOV-GP (Fig. 1a), as expected (4, 24). Notably, expression of the FLUAV-activating TTSPs, TMPRSS2, TMPRSS4 and HAT, also abrogated inhibition of VLP release by tetherin, while no rescue was observed upon coexpression of TMPRSS3 and TMPRSS6 (Fig. 1A), which fail to activate FLUAV (42). Analysis of a catalytically inactive mutant of TMPRSS2 revealed that tetherin antagonism was dependent on the protease activity (Fig. 1B) and the robust and comparable expression of epitope-tagged proteases in the transfected cells (Fig. 1C) revealed that lack of anti-tetherin activity of TMPRSS3 and TMPRSS6 was not due to insufficient expression. Thus, the FLUAV-activating proteases TMPRSS2, TMPRSS4 and HAT can apparently antagonize the antiviral activity of tetherin.

FLUAV activating type II transmembrane serine proteases differentially interfere with tetherin expression. Tetherin antagonism by TTSPs might result from interference with 
appropriate tetherin expression in cells and at the cell surface, as previously demonstrated for HIV-1 Vpu (4). Coexpression of Vpu indeed reduced total tetherin expression and cell surface levels (Fig. 2A,B), as expected (4). In contrast, tetherin levels were not modulated by coexpression of EBOV-GP (Fig. 2A,B), again in agreement with published work $(26,61)$. Similarly, tetherin expression remained largely unchanged upon coexpression of most TTSPs. The only exception was observed upon TMPRSS4 expression, which markedly reduced intracellular levels of tetherin (Fig. 2A) but, for at present unclear reasons, had little if any impact on cell surface levels of tetherin (Fig. 2B). Similarly, expression of the other TTSPs analyzed had no significant effects on surface expression. These observations indicate that TMPRSS2 and HAT antagonize tetherin without appreciably modulating tetherin expression while TMPRSS4 robustly reduces total tetherin expression.

FLUAV activating type II transmembrane serine proteases cleave tetherin. The finding that some TTSPs can antagonize tetherin without interfering with tetherin expression prompted us to investigate whether TTSPs facilitate tetherin proteolysis, which can compromise tetherin's antiviral activity $(13,15,43)$. To address this question, we examined whether tetherin fragments are present in supernatants of cells coexpressing TTSPs and Gag. For this endeavor, we extended our TTSP panel to hepsin, DESC1 and MSPL, which can cleave HA (46), and to TMPRSS1B, TMPRSS11F, prostasin, TMPRSS9 and TMPRSS10, which are unable to process HA despite robust expression in 293T cells (46). All TTSPs known to cleave and activate HA liberated Gag release from inhibition by tetherin and robust tetherin signals of approximately $20 \mathrm{kDa}$ were readily observed in the supernatants of cells coexpressing TMPRSS2, MSPL and hepsin (Fig. 3A). In addition, tetherin fragments were also observed upon coexpression of TMPRSS4 and HAT in some experiments (not shown). In contrast, proteases unable to activate HA consistently failed to antagonize tetherin and to 
facilitate release of tetherin fragments into the extracellular space (Fig. 3A). Similarly, coexpression of EBOV-GP and Vpu did not result in efficient release of tetherin fragments. Moreover, in some but not all experiments coexpression of TMPRSS2, hepsin and MSPL resulted in the accumulation of a tetherin form in cell lysates which displayed the same molecular weight as the tetherin fragment observed in supernatants (Fig. 3A). Collectively, these findings suggested that TTSPs might cleave and thereby antagonize tetherin.

We next sought to provide direct proof for tetherin cleavage. For this, we focused on experiments in which faint tetherin signals were detected in supernatants of control cells and EBOV-GP expressing cells, potentially due to particularly high transfection efficiency. PNGase F digest, which removes all N-linked glycans, showed that the diffuse tetherin signals in these supernatants were due to heterogenous glycosylation of tetherin (Fig. 3B). More importantly, removal of $\mathrm{N}$-glycans revealed the presence of an approximately $14 \mathrm{kDa}$ tetherin fragment in supernatants from cells coexpressing TMPRSS2 but not EBOV-GP or empty plasmid (Fig. 3B), indicating that TMPRSS2 indeed cleaves tetherin. Thus, our observations are consistent with a model, in which tetherin cleavage by TTSPs, in analogy to tetherin proteolysis by trypsin, mediates the release of otherwise surface-tethered virions into the extracellular space.

TMPRSS2 and tetherin colocalize. The ability of HA-activating TTSPs to cleave tetherin suggests that TTSPs and tetherin might colocalize. Confocal microscopy of cells coexpressing tetherin and TTSPs fused to fluorescent proteins indeed revealed extensive colocalization of tetherin and TMPRSS2 at the plasma membrane and within cytoplasmic compartments (Fig. 4A) In contrast, colocalization of tetherin and TMPRSS6 was much less robust (Fig. 4A). Similar results were obtained when the potential interaction of these proteins was studied employing a previously described FACS-based FRET assay (55), for 
which expression of CFP and YFP alone served as negative control while CFP fused to YFP served as positive control (Fig. 4B). In this assay, a robust FRET signal was measured upon coexpression of $\mathrm{Vpu}$ and tetherin, as expected (55), and upon coexpression of tetherin and TMPRSS2 (Fig. 4B). In contrast, FRET in tetherin and TMPRSS6 transfected cells was much lower and no appreciable signal was detected when the proteases were coexpressed with the fluorescent proteins fused to a membrane targeting signal (Fig. 4B). Thus, TMPRSS2, like $\mathrm{Vpu}$, is in the same subcellular compartment and directly interacts with tetherin.

Endogenous TMPRSS2 promotes release of retroviral particles from Caco-2 cells. Our analysis so far showed tetherin counteraction by TTSPs in transfected cells. In order to examine if tetherin antagonism is also operative upon endogenous expression of protease and tetherin, we analyzed release of wt and vpu-defective HIV-1 from the colon carcinoma cell line Caco-2. Caco-2 cells were chosen for our analysis because these cells express endogenous tetherin (Fig. 5) and TMPRSS2 and support influenza virus spread in a trypsinindependent, TMPRSS2-dependent manner $(51,62)$. Transfection of Caco-2 cells with tetherin-specific siRNA resulted in a marked knock-down of tetherin expression (Fig. 5). Lack of efficient detection of TMPRSS2 expression by Western blot precluded a similar analysis for TMPRSS2-specific siRNA. However, TMPRSS2 transcripts are abundant in Caco-2 cells $(51,63)$ and our previous study conducted with a different batch of Caco-2 cells clearly demonstrated that the siRNA employed here effectively and specifically reduces TMPRSS2 expression in this cell line (51). Knock-down of tetherin expression in cells containing HIV-1 proviral DNA slightly increased release of wt HIV-1 while knock-down of TMPRSS2 expression modestly reduced viral release (Fig. 5), in keeping with tetherin antagonism by this protease. More strikingly, release of vpu-negative HIV-1 was markedly enhanced upon knock-down of tetherin but completely inhibited upon reduction of 
TMPRSS2 expression (Fig. 5), consistent with the concept that TMPRSS2 promotes viral release by negatively regulating tetherin's antiviral activity.

\section{TMPRSS2 promotes release of authentic influenza A viruses from tetherin expressing}

cells. Our analysis was so far based on the use of HIV-1 VLPs. This system is frequently employed to study tetherin but might not faithfully reflect FLUAV inhibition by tetherin. Therefore, we next investigated whether tetherin inhibits release of FLUAV-like particles and infectious FLUAV and whether this inhibition can be counteracted by TMPRSS2.

Transfection of the NA of the 1918 FLUAV into Caco- 2 cells resulted in the robust release of NA into the supernatant (Fig. 6A), in agreement with previous findings that NA triggers release of VLPs $(40,64)$. Release of NA-based VLPs was increased upon siRNA-mediated down-regulation of tetherin expression while knock-down of TMPRSS2 expression reduced viral release (Fig. 6A). These results mirror our observations made with retroviral VLPs and suggest that TMPRSS2 might promote FLUAV release from tetherin positive target cells. In order to investigate this, we analyzed viral release from cells transfected to express tetherin or control transfected with empty plasmid, employing a single-cycle of infection assay. We found that tetherin expression reduced the release of A/PR/8/34 (H1N1) and A/Panama/2007/99 (H3N2) strains by roughly 10-fold (Fig. 6B) and this effect was not observed when a tetherin mutant with deleted transmembrane domain was used (Fig. 6C). Notably, inhibition of viral release by tetherin was fully rescued upon coexpression of TMPRSS2 while expression of TMPRSS2 alone did not impact viral release (Fig. 6D). For this experiment, supernatants were collected at $8 \mathrm{~h}$ (Fig. 6D) instead of $24 \mathrm{~h}$ (Fig. 6B,C) post infection, in order to avoid multiple-rounds of infection supported by TMPRSS2-mediated HA activation. As a consequence, less infectious units were present in the supernatants assayed in figure 6D as compared to previously analyzed supernatants (Fig. 6B,C). However, 
supernatants taken at $1 \mathrm{~h}$ post infection generally did not contain infectivity (not shown), indicating that the infectious units detected at $8 \mathrm{~h}$ time point were due to release of progeny virions. Finally, siRNA knock-down of endogenous tetherin in Caco-2 cells subtly increased viral release in a single-cycle assay (not shown) but markedly augmented multi-cycle FLUAV spread while knock-down of TMPRSS2 had the opposite effects (Fig. 6E). Thus, TMPRSS2 might counteract tetherin in cells endogenously expressing these proteins, although reduced viral spread upon TMPRSS2 knock-down might be partially due to diminished HA activation. Collectively, these results suggest that exogenous and likely also endogenous TMPRSS2 can rescue FLUAV from inhibition by tetherin.

TMPRSS2 and tetherin are coexpressed in human bronchial epithelium. A tetherin antagonizing activity of TMPRSS2 in the context of the infected host requires coexpression of protease and tetherin in viral target cells. Human bronchial epithelial cells express TMPRSS2 and the major receptor determinant for human influenza viruses, 2,6-linked sialic acid (52), and are permissive to influenza virus spread. Therefore, we analyzed if TMPRSS2 and tetherin are coexpressed in the human bronchus. Immunofluorescence analysis of antibody-stained bronchial tissue demonstrated that bronchial epithelial cells, identified by their location in the analyzed tissue and by the prominent expression of $\beta$-tubulin, coexpress robust levels of tetherin and TMPRSS2 (Fig. 7). Thus, TMPRSS2 and tetherin are coexpressed in influenza virus target cells in the respiratory tract. 


\section{DISCUSSION}

Understanding how tetherin exerts antiviral activity and how viruses evade inhibition by tetherin might allow for novel approaches to antiviral intervention and has been in the focus of recent research. The paradigm that enveloped viruses which bud from the cell surface are either inhibited by tetherin or encode a robust tetherin antagonizing activity has been verified for viruses from diverse families $(4,17-19,24,65,66)$. For unclear reasons, FLUAV constitutes an exception to this rule (34-40). Our results indicate that FLUAV can acquire tetherin resistance by targeting cells which express tetherin antagonizing, HA-activating proteases. To our knowledge this is the first report of a cellular tetherin antagonist which can be exploited by viruses for efficient release from tetherin-positive cells.

The influenza virus HA mediates viral entry into target cells. Activation of HA by host cell proteases is essential for viral infectivity but the enzymes which facilitate this process in the infected host have long been unclear (42). Recent analyses indicate a key role of TTSPs: In transfected cells TMPRSS2 and HAT activate the HA-proteins of all FLUAV subtypes previously pandemic in humans (44), endogenous TMPRSS2 activates influenza viruses for spread in Calu-3 (67) and Caco-2 cells (51) and TMPRSS2 and HAT are expressed in viral target cells in the aerodigestive tract (52). Moreover, a pioneer study by Hatesuer and colleagues demonstrated that TMPRSS2 expression is essential for spread and pathogenesis of $\mathrm{H} 1 \mathrm{~N} 1$ viruses in mice (47) and these results were confirmed by subsequent work $(48,49)$. Similarly, polymorphisms in TMPRSS2 which increase expression of this protease were shown to be associated with severe influenza in humans (68). In addition, hepatitis C virus (69), human metapneumovirus (70), respiratory parainfluenzaviruses (71) and several respiratory coronaviruses, including the highly pathogenic severe acute respiratory syndrome (72-75) and Middle East respiratory syndrome coronaviruses $(71,76)$, are activated by TMPRSS 2 and might exploit this protease to ensure their spread in the 
infected host. Our present results suggest that adaption to usage of TMPRSS2 and other TTSPs for activation of the viral surface protein might be associated with an additional benefit - protection from the antiviral action of tetherin.

How do TTSPs antagonize tetherin? Our observations indicate that TTSPs could sever the tether between viral and host cell membrane, which impedes release of progeny virions from tetherin-positive cells. Such a mechanism would fit with the documented ability of trypsin to cleave and antagonize tetherin $(13,15,43)$. In fact, TTSPs display a trypsin-like specificity and it is conceivable that both proteases cleave tetherin at identical sites. TTSPs are autocatalytically activated during transport to or upon insertion into the plasma membrane (77) and it is likely that these proteases cleave tetherin at the cell surface. Processing of tetherin by TMPRSS4 might be an exception. The profound depletion of total tetherin levels by this protease suggests that TMPRSS4 can also cleave tetherin during transport in the constitutive secretory pathway and thereby largely depletes intracellular tetherin pools. However, depletion is not complete since TMPRSS4 expression does not affect surface tetherin levels. In sum, tetherin joins influenza HA and coronavirus spike proteins as a TTSP substrate and tetherin cleavage is most likely responsible for tetherin counteraction by TTSPs.

The demonstration that tetherin can robustly inhibit release of authentic, laboratory adapted and seasonal FLUAV is in agreement with work by some laboratories $(37,38)$ but does not match findings of three other studies, including our own work, demonstrating that tetherin has at best a modest effect on FLUAV release (34-36). One explanation for these discrepant results could be that the cells used for these studies differed in tetherin expression levels. A second parameter which could impact FLUAV inhibition by tetherin is the presence of trypsin, which activates FLUAV but inactivates tetherin $(13,15,43)$. To avoid such a potentially confounding effect, we analyzed the antiviral activity of tetherin in the absence of 
trypsin. In addition, FLUAV infection experiments involving coexpression of TMPRSS2 and tetherin were terminated at $8 \mathrm{~h}$ post infection (except for figure 6e), in order to ensure that rescue of FLUAV from inhibition by tetherin was due to tetherin inactivation by TMPRSS2 and not to augmented viral spread as a consequence of HA activation by this protease. In sum, tetherin can inhibit FLUAV release and this inhibition is abrogated byTMPRSS2.

Is tetherin able to inhibit FLUAV in the infected host and is this inhibition rescued by TMPRSS2? Our analysis of TMPRSS2 and tetherin expression in human bronchial epithelium suggests that both might be the case. The expression of tetherin in epithelial cells of the human bronchus is in agreement with a previous study demonstrating constitutive expression of tetherin in many organs, including lung (78). TMPRSS2 is known to be coexpressed with 2,6-linked sialic acid throughout the respiratory tract (52) and the present finding that TMPRSS2 and tetherin are coexpressed in bronchial epithelium demonstrates that TTSPs could promote influenza virus spread in tetherin-positive pulmonary target cells a scenario in keeping with the observation that tetherin knock-out does not augment spread of FLUBV in mice (79). Importantly, the biological relevance of tetherin antagonism by TTSPs might extend beyond the spread and pathogenesis of respiratory viruses which target TTSPpositive cells. For one, the catalytic domain of several TTSPs is shed into the extracellular space $(77,80)$ and might inactivate tetherin on adjacent cells. Moreover, tetherin-inactivating TTSPs are expressed in extrapulmonary tissues and might determine whether viruses targeting these tissues are exposed to tetherin's antiviral activity (77). Thus, TMPRSS2 expression in lymphocytes (52) might impact release of HIV and SIV from these cells and evidence was obtained that TMPRSS2 in hepatocytes can activate HCV for host cell entry. Finally, inflammatory conditions can increase the activation of certain TTSPs (81) and can induce the expression of other proteases (82), which might counter tetherin. 
Inhibition of virus activating cellular proteases is a promising approach to antiviral therapy and enzymes like TMPRSS2, which is important for viral spread $(47,48,83)$ but dispensable for cellular survival (84) are important targets. Our study suggests that inhibitors like the IFN-induced serine protease inhibitor PAI-1, which targets TMPRSS2 and related proteases (39), might exert antiviral activity via two mechanisms: Inhibition of viral activation and augmentation of viral inhibition by tetherin. 


\section{ACKNOWLEDGEMENTS}

We thank Anika Lins and Inga Nehlmeier for excellent technical assistance. The following reagents were obtained through the NIH AIDS Reagent Program, Division of AIDS, NIAID, NIH: HIV-1 p24 hybridoma (183-H12-5C) from Dr. Bruce Chesebro and anti-Bst-2 (cat\# 11721) from Drs. Klaus Strebel and Amy Andrew. The authors declare that there are no conflicts of interest.P.Z. was supported by the Leibniz Graduate School Emerging Infectious Diseases, S.P. was supported by the Leibniz Gemeinschaft and the DFG (716/6-1, 716/8-1). V.T. and R.D. were supported by the Swiss National Science Foundation (project 31003A_132898; V.T.), the 3R Research Foundation Switzerland (project 128-11; V.T. and R.D.), and the Deutsche Forschungsgemeinschaft (Priority Program (SPP) 1596, V.T.; SFB900, C.B.K, S.P.). T.W. acknowledges support from the Deutsche Forschungsgemeinschaft (TransRegio84 and WO 554/4-1). M.S. was supported by the Deutsche Forschungsgemeinschaft (Schi1073/4-1). K.G. was supported by the HBRS program DEWIN. This work was partly supported by DFG (PO 716/6-1) and the Göttingen Graduate School for Neurosciences, Biophysics, and Molecular Biosciences (DFG Grant GSC 226/1 and DFG Grant GSC 226/2). 


\section{REFERENCES}

1. Yan, N. and Z. J. Chen. 2012. Intrinsic antiviral immunity. Nat. Immunol. 13:214222.

2. Blanco-Melo, D., S. Venkatesh, and P. D. Bieniasz. 2012. Intrinsic cellular defenses against human immunodeficiency viruses. Immunity. 37:399-411.

3. Neil, S. J. 2013. The antiviral activities of tetherin. Curr. Top. Microbiol. Immunol. 371:67-104.

4. Neil, S. J., T. Zang, and P. D. Bieniasz. 2008. Tetherin inhibits retrovirus release and is antagonized by HIV-1 Vpu. Nature 451:425-430.

5. Van, D. N., D. Goff, C. Katsura, R. L. Jorgenson, R. Mitchell, M. C. Johnson, E. B. Stephens, and J. Guatelli. 2008. The interferon-induced protein BST-2 restricts HIV-1 release and is downregulated from the cell surface by the viral Vpu protein. Cell Host. Microbe 3:245-252.

6. Barrett, B. S., D. S. Smith, S. X. Li, K. Guo, K. J. Hasenkrug, and M. L. Santiago. 2012. A single nucleotide polymorphism in tetherin promotes retrovirus restriction in vivo. PLoS. Pathog. 8:e1002596.

7. Jones, P. H., H. V. Mehta, M. Maric, R. J. Roller, and C. M. Okeoma. 2012. Bone marrow stromal cell antigen 2 (BST-2) restricts mouse mammary tumor virus (MMTV) replication in vivo. Retrovirology. 9:10.

8. Li, S. X., B. S. Barrett, K. J. Heilman, R. J. Messer, R. A. Liberatore, P. D. Bieniasz, G. Kassiotis, K. J. Hasenkrug, and M. L. Santiago. 2014. Tetherin promotes the innate and adaptive cell-mediated immune response against retrovirus infection in vivo. J. Immunol. 193:306-316.

9. Dave, V. P., F. Hajjar, M. M. Dieng, E. Haddad, and E. A. Cohen. 2013. Efficient BST2 antagonism by Vpu is critical for early HIV-1 dissemination in humanized mice. Retrovirology. 10:128.

10. Weissenhorn, W., N. Miguet, N. Aschman, P. Renesto, Y. Usami, and H. G. Gottlinger. 2012. Structural basis of tetherin function. Curr. HIV. Res. 10:298-306.

11. Kupzig, S., V. Korolchuk, R. Rollason, A. Sugden, A. Wilde, and G. Banting. 2003. Bst-2/HM1.24 is a raft-associated apical membrane protein with an unusual topology. Traffic. 4:694-709.

12. Perez-Caballero, D., T. Zang, A. Ebrahimi, M. W. McNatt, D. A. Gregory, M. C. Johnson, and P. D. Bieniasz. 2009. Tetherin inhibits HIV-1 release by directly tethering virions to cells. Cell 139:499-511.

13. Venkatesh, S. and P. D. Bieniasz. 2013. Mechanism of HIV-1 Virion Entrapment by Tetherin. PLoS. Pathog. 9:e1003483. 
14. Fitzpatrick, K., M. Skasko, T. J. Deerinck, J. Crum, M. H. Ellisman, and J. Guatelli. 2010. Direct restriction of virus release and incorporation of the interferoninduced protein BST-2 into HIV-1 particles. PLoS. Pathog. 6:e1000701.

15. Hammonds, J., J. J. Wang, H. Yi, and P. Spearman. 2010. Immunoelectron microscopic evidence for Tetherin/BST2 as the physical bridge between HIV-1 virions and the plasma membrane. PLoS. Pathog. 6:e1000749.

16. Van Damme N., D. Goff, C. Katsura, R. L. Jorgenson, R. Mitchell, M. C. Johnson, E. B. Stephens, and J. Guatelli. 2008. The interferon-induced protein BST-2 restricts HIV-1 release and is downregulated from the cell surface by the viral Vpu protein. Cell Host. Microbe 3:245-252.

17. Jia, B., R. Serra-Moreno, W. Neidermyer, A. Rahmberg, J. Mackey, I. B. Fofana, W. E. Johnson, S. Westmoreland, and D. T. Evans. 2009. Species-specific activity of SIV Nef and HIV-1 Vpu in overcoming restriction by tetherin/BST2. PLoS. Pathog. 5:e1000429.

18. Zhang, F., S. J. Wilson, W. C. Landford, B. Virgen, D. Gregory, M. C. Johnson, J. Munch, F. Kirchhoff, P. D. Bieniasz, and T. Hatziioannou. 2009. Nef proteins from simian immunodeficiency viruses are tetherin antagonists. Cell Host. Microbe 6:54-67.

19. Le, T. A. and S. J. Neil. 2009. Antagonism to and intracellular sequestration of human tetherin by the human immunodeficiency virus type 2 envelope glycoprotein. J. Virol. 83:11966-11978.

20. Goffinet, C., S. Schmidt, C. Kern, L. Oberbremer, and O. T. Keppler. 2010. Endogenous CD317/Tetherin limits replication of HIV-1 and murine leukemia virus in rodent cells and is resistant to antagonists from primate viruses. J. Virol. 84:1137411384.

21. Kong, W. S., T. Irie, A. Yoshida, R. Kawabata, T. Kadoi, and T. Sakaguchi. 2012. Inhibition of virus-like particle release of Sendai virus and Nipah virus, but not that of mumps virus, by tetherin/CD317/BST-2. Hiroshima J. Med. Sci. 61:59-67.

22. Radoshitzky, S. R., L. Dong, X. Chi, J. C. Clester, C. Retterer, K. Spurgers, J. H. Kuhn, S. Sandwick, G. Ruthel, K. Kota, D. Boltz, T. Warren, P. J. Kranzusch, S. P. Whelan, and S. Bavari. 2010. Infectious Lassa virus, but not filoviruses, is restricted by BST-2/tetherin. J. Virol. 84:10569-10580.

23. Jouvenet, N., S. J. Neil, M. Zhadina, T. Zang, Z. Kratovac, Y. Lee, M. McNatt, T. Hatziioannou, and P. D. Bieniasz. 2009. Broad-spectrum inhibition of retroviral and filoviral particle release by tetherin. J. Virol. 83:1837-1844.

24. Kaletsky, R. L., J. R. Francica, C. Agrawal-Gamse, and P. Bates. 2009. Tetherinmediated restriction of filovirus budding is antagonized by the Ebola glycoprotein. Proc. Natl. Acad. Sci. U. S. A 106:2886-2891.

25. Sakuma, T., T. Noda, S. Urata, Y. Kawaoka, and J. Yasuda. 2009. Inhibition of Lassa and Marburg virus production by tetherin. J. Virol. 83:2382-2385. 
26. Kühl, A., C. Banning, A. Marzi, J. Votteler, I. Steffen, S. Bertram, I. Glowacka, A. Konrad, M. Sturzl, J. T. Guo, U. Schubert, H. Feldmann, G. Behrens, M. Schindler, and S. Pohlmann. 2011. The Ebola virus glycoprotein and HIV-1 Vpu employ different strategies to counteract the antiviral factor tetherin. J. Infect. Dis. 204 Suppl 3:S850-S860.

27. Mansouri, M., K. Viswanathan, J. L. Douglas, J. Hines, J. Gustin, A. V. Moses, and K. Fruh. 2009. Molecular mechanism of BST2/tetherin downregulation by K5/MIR2 of Kaposi's sarcoma-associated herpesvirus. J. Virol. 83:9672-9681.

28. Pan, X. B., X. W. Qu, D. Jiang, X. L. Zhao, J. C. Han, and L. Wei. 2013. BST2/Tetherin inhibits hepatitis $\mathrm{C}$ virus production in human hepatoma cells. Antiviral Res. 98:54-60.

29. Dafa-Berger, A., A. Kuzmina, M. Fassler, H. Yitzhak-Asraf, Y. Shemer-Avni, and R. Taube. 2012. Modulation of hepatitis $C$ virus release by the interferoninduced protein BST-2/tetherin. Virology 428:98-111.

30. Jones, P. H., M. Maric, M. N. Madison, W. Maury, R. J. Roller, and C. M. Okeoma. 2013. BST-2/tetherin-mediated restriction of chikungunya (CHIKV) VLP budding is counteracted by CHIKV non-structural protein 1 (nsP1). Virology 438:3749.

31. Sakuma, T., A. Sakurai, and J. Yasuda. 2009. Dimerization of tetherin is not essential for its antiviral activity against Lassa and Marburg viruses. PLoS. One. 4:e6934.

32. Rodriguez-Boulan, E., K. T. Paskiet, P. J. Salas, and E. Bard. 1984. Intracellular transport of influenza virus hemagglutinin to the apical surface of Madin-Darby canine kidney cells. J. Cell Biol. 98:308-319.

33. Rossman, J. S. and R. A. Lamb. 2011. Influenza virus assembly and budding. Virology 411:229-236.

34. Bruce, E. A., T. E. Abbink, H. M. Wise, R. Rollason, R. P. Galao, G. Banting, S. J. Neil, and P. Digard. 2012. Release of filamentous and spherical influenza A virus is not restricted by tetherin. J. Gen. Virol. 93:963-969.

35. Watanabe, R., G. P. Leser, and R. A. Lamb. 2011. Influenza virus is not restricted by tetherin whereas influenza VLP production is restricted by tetherin. Virology 417:50-56.

36. Winkler, M., S. Bertram, K. Gnirss, I. Nehlmeier, A. Gawanbacht, F. Kirchhoff, C. Ehrhardt, S. Ludwig, M. Kiene, A. S. Moldenhauer, U. Goedecke, C. B. Karsten, A. Kuhl, and S. Pohlmann. 2012. Influenza A virus does not encode a tetherin antagonist with Vpu-like activity and induces IFN-dependent tetherin expression in infected cells. PLoS. One. 7:e43337.

37. Mangeat, B., L. Cavagliotti, M. Lehmann, G. Gers-Huber, I. Kaur, Y. Thomas, L. Kaiser, and V. Piguet. 2012. Influenza virus partially counteracts restriction imposed by tetherin/BST-2. J. Biol. Chem. 287:22015-22029. 
38. Leyva-Grado, V. H., R. Hai, F. Fernandes, A. Belicha-Villanueva, C. Carter, and M. A. Yondola. 2014. Modulation of an ectodomain motif in the influenza A virus neuraminidase alters tetherin sensitivity and results in virus attenuation in vivo. J. Mol. Biol. 426:1308-1321.

39. Dittmann, M., H. H. Hoffmann, M. A. Scull, R. H. Gilmore, K. L. Bell, M. Ciancanelli, S. J. Wilson, S. Crotta, Y. Yu, B. Flatley, J. W. Xiao, J. L. Casanova, A. Wack, P. D. Bieniasz, and C. M. Rice. 2015. A serpin shapes the extracellular environment to prevent influenza a virus maturation. Cell 160:631-643.

40. Yondola, M. A., F. Fernandes, A. Belicha-Villanueva, M. Uccelini, Q. Gao, C. Carter, and P. Palese. 2011. Budding capability of the influenza virus neuraminidase can be modulated by tetherin. J. Virol. 85:2480-2491.

41. Gnirss, K., P. Zmora, P. Blazejewska, M. Winkler, A. Lins, I. Nehlmeier, S. Gartner, A. S. Moldenhauer, H. Hofmann-Winkler, T. Wolff, M. Schindler, and S. Pohlmann. 2015. Tetherin sensitivity of influenza A viruses is strain specific: Role of hemagglutinin and neuraminidase. J. Virol.

42. Bertram, S., I. Glowacka, I. Steffen, A. Kuhl, and S. Pohlmann. 2010. Novel insights into proteolytic cleavage of influenza virus hemagglutinin. Rev. Med. Virol. 20:298-310.

43. Neil, S. J., V. Sandrin, W. I. Sundquist, and P. D. Bieniasz. 2007. An interferonalpha-induced tethering mechanism inhibits HIV-1 and Ebola virus particle release but is counteracted by the HIV-1 Vpu protein. Cell Host. Microbe 2:193-203.

44. Böttcher, E., T. Matrosovich, M. Beyerle, H. D. Klenk, W. Garten, and M. Matrosovich. 2006. Proteolytic activation of influenza viruses by serine proteases TMPRSS2 and HAT from human airway epithelium. J. Virol. 80:9896-9898.

45. Chaipan, C., D. Kobasa, S. Bertram, I. Glowacka, I. Steffen, T. S. Tsegaye, M. Takeda, T. H. Bugge, S. Kim, Y. Park, A. Marzi, and S. Pohlmann. 2009. Proteolytic activation of the 1918 influenza virus hemagglutinin. J. Virol. 83:32003211.

46. Zmora, P., P. Blazejewska, A. S. Moldenhauer, K. Welsch, I. Nehlmeier, Q. Wu, H. Schneider, S. Pohlmann, and S. Bertram. 2014. DESC1 and MSPL activate influenza A viruses and emerging coronaviruses for host cell entry. J. Virol. 88:12087-12097.

47. Hatesuer, B., S. Bertram, N. Mehnert, M. M. Bahgat, P. S. Nelson, S. Pohlman, and K. Schughart. 2013. Tmprss2 is essential for influenza H1N1 virus pathogenesis in mice. PLoS. Pathog. 9:e1003774.

48. Sakai, K., Y. Ami, M. Tahara, T. Kubota, M. Anraku, M. Abe, N. Nakajima, T. Sekizuka, K. Shirato, Y. Suzaki, A. Ainai, Y. Nakatsu, K. Kanou, K. Nakamura, T. Suzuki, K. Komase, E. Nobusawa, K. Maenaka, M. Kuroda, H. Hasegawa, Y. Kawaoka, M. Tashiro, and M. Takeda. 2014. The host protease TMPRSS2 plays a major role in in vivo replication of emerging H7N9 and seasonal influenza viruses. J. Virol. 88:5608-5616. 
49. Tarnow, C., G. Engels, A. Arendt, F. Schwalm, H. Sediri, A. Preuss, P. S. Nelson, W. Garten, H. D. Klenk, G. Gabriel, and E. Bottcher-Friebertshauser. 2014. TMPRSS2 is a host factor that is essential for pneumotropism and pathogenicity of H7N9 influenza A virus in mice. J. Virol. 88:4744-4751.

50. Kühl, A., J. Münch, D. Sauter, S. Bertram, I. Glowacka, I. Steffen, A. Specht, H. Hofmann, H. Schneider, G. Behrens, and S. Pöhlmann. 2010. Calcium-modulating cyclophilin ligand does not restrict retrovirus release. Nat. Med. 16:155-156.

51. Bertram, S., I. Glowacka, P. Blazejewska, E. Soilleux, P. Allen, S. Danisch, I. Steffen, S. Y. Choi, Y. Park, H. Schneider, K. Schughart, and S. Pohlmann. 2010. TMPRSS2 and TMPRSS4 facilitate trypsin-independent spread of influenza virus in Caco-2 cells. J. Virol. 84:10016-10025.

52. Bertram, S., A. Heurich, H. Lavender, S. Gierer, S. Danisch, P. Perin, J. M. Lucas, P. S. Nelson, S. Pohlmann, and E. J. Soilleux. 2012. Influenza and SARScoronavirus activating proteases TMPRSS2 and HAT are expressed at multiple sites in human respiratory and gastrointestinal tracts. PLoS. One. 7:e35876.

53. Heurich, A., H. Hofmann-Winkler, S. Gierer, T. Liepold, O. Jahn, and S. Pohlmann. 2013. TMPRSS2 and ADAM17 cleave ACE2 differentially and only proteolysis by TMPRSS 2 augments entry driven by the SARS-coronavirus spikeprotein. J. Virol.

54. Matthaei, M., M. Budt, and T. Wolff. 2013. Highly pathogenic H5N1 influenza A virus strains provoke heterogeneous IFN-alpha/beta responses that distinctively affect viral propagation in human cells. PLoS. One. 8:e56659.

55. Banning, C., J. Votteler, D. Hoffmann, H. Koppensteiner, M. Warmer, R. Reimer, F. Kirchhoff, U. Schubert, J. Hauber, and M. Schindler. 2010. A flow cytometry-based FRET assay to identify and analyse protein-protein interactions in living cells. PLoS. One. 5:e9344.

56. Miyagi, E., A. J. Andrew, S. Kao, and K. Strebel. 2009. Vpu enhances HIV-1 virus release in the absence of Bst-2 cell surface down-modulation and intracellular depletion. Proc. Natl. Acad. Sci. U. S. A 106:2868-2873.

57. Chesebro, B., K. Wehrly, J. Nishio, and S. Perryman. 1992. Macrophage-tropic human immunodeficiency virus isolates from different patients exhibit unusual V3 envelope sequence homogeneity in comparison with T-cell-tropic isolates: definition of critical amino acids involved in cell tropism. J. Virol. 66:6547-6554.

58. Srivastava, B., P. Blazejewska, M. Hessmann, D. Bruder, R. Geffers, S. Mauel, A. D. Gruber, and K. Schughart. 2009. Host genetic background strongly influences the response to influenza a virus infections. PLoS. One. 4:e4857.

59. Hagen, N., K. Bayer, K. Rosch, and M. Schindler. 2014. The intraviral protein interaction network of hepatitis C virus. Mol. Cell Proteomics. 13:1676-1689.

60. Dijkman, R., M. F. Jebbink, S. M. Koekkoek, M. Deijs, H. R. Jonsdottir, R. Molenkamp, M. Ieven, H. Goossens, V. Thiel, and L. van der Hoek. 2013. Isolation and characterization of current human coronavirus strains in primary human 
epithelial cell cultures reveal differences in target cell tropism. J. Virol. 87:60816090.

61. Lopez, L. A., S. J. Yang, H. Hauser, C. M. Exline, K. G. Haworth, J. Oldenburg, and P. M. Cannon. 2010. Ebola virus glycoprotein counteracts BST-2/Tetherin restriction in a sequence-independent manner that does not require tetherin surface removal. J. Virol. 84:7243-7255.

62. Zhirnov, O. and H. D. Klenk. 2003. Human influenza A viruses are proteolytically activated and do not induce apoptosis in CACO-2 cells. Virology 313:198-212.

63. Bertram, S., R. Dijkman, M. Habjan, A. Heurich, S. Gierer, I. Glowacka, K. Welsch, M. Winkler, H. Schneider, H. Hofmann-Winkler, V. Thiel, and S. Pohlmann. 2013. TMPRSS2 activates the human coronavirus 229E for cathepsinindependent host cell entry and is expressed in viral target cells in the respiratory epithelium. J. Virol. 87:6150-6160.

64. Lai, J. C., W. W. Chan, F. Kien, J. M. Nicholls, J. S. Peiris, and J. M. Garcia. 2010. Formation of virus-like particles from human cell lines exclusively expressing influenza neuraminidase. J. Gen. Virol. 91:2322-2330.

65. Bampi, C., L. Rasga, and L. Roux. 2013. Antagonism to human BST-2/tetherin by Sendai virus glycoproteins. J. Gen. Virol. 94:1211-1219.

66. Bartee, E., A. McCormack, and K. Fruh. 2006. Quantitative membrane proteomics reveals new cellular targets of viral immune modulators. PLoS. Pathog. 2:e107.

67. Böttcher-Friebertshauser, E., D. A. Stein, H. D. Klenk, and W. Garten. 2011. Inhibition of influenza virus infection in human airway cell cultures by an antisense peptide-conjugated morpholino oligomer targeting the hemagglutinin-activating protease TMPRSS2. J. Virol. 85:1554-1562.

68. Cheng, Z., J. Zhou, K. K. To, H. Chu, C. Li, D. Wang, D. Yang, S. Zheng, K. Hao, Y. Bosse, M. Obeidat, C. A. Brandsma, Y. Q. Song, Y. Chen, B. J. Zheng, L. Li, and K. Y. Yuen. 2015. Identification of TMPRSS2 as a Susceptibility Gene for Severe 2009 Pandemic A(H1N1) Influenza and A(H7N9) Influenza. J. Infect. Dis.

69. Esumi, M., M. Ishibashi, H. Yamaguchi, S. Nakajima, Y. Tai, S. Kikuta, M. Sugitani, T. Takayama, M. Tahara, M. Takeda, and T. Wakita. 2015. Transmembrane serine protease TMPRSS2 activates hepatitis $\mathrm{C}$ virus infection. Hepatology 61:437-446.

70. Shirogane, Y., M. Takeda, M. Iwasaki, N. Ishiguro, H. Takeuchi, Y. Nakatsu, M. Tahara, H. Kikuta, and Y. Yanagi. 2008. Efficient multiplication of human metapneumovirus in Vero cells expressing the transmembrane serine protease TMPRSS2. J. Virol. 82:8942-8946.

71. Shirato, K., M. Kawase, and S. Matsuyama. 2013. Middle East respiratory syndrome coronavirus infection mediated by the transmembrane serine protease TMPRSS2. J. Virol. 87:12552-12561. 
72. Glowacka, I., S. Bertram, M. A. Muller, P. Allen, E. Soilleux, S. Pfefferle, I. Steffen, T. S. Tsegaye, Y. He, K. Gnirss, D. Niemeyer, H. Schneider, C. Drosten, and S. Pohlmann. 2011. Evidence that TMPRSS2 activates the severe acute respiratory syndrome coronavirus spike protein for membrane fusion and reduces viral control by the humoral immune response. J. Virol. 85:4122-4134.

73. Matsuyama, S., N. Nagata, K. Shirato, M. Kawase, M. Takeda, and F. Taguchi. 2010. Efficient activation of the severe acute respiratory syndrome coronavirus spike protein by the transmembrane protease TMPRSS2. J. Virol. 84:12658-12664.

74. Shulla, A., T. Heald-Sargent, G. Subramanya, J. Zhao, S. Perlman, and T. Gallagher. 2011. A transmembrane serine protease is linked to the severe acute respiratory syndrome coronavirus receptor and activates virus entry. J. Virol. 85:873882.

75. Zhou, Y., P. Vedantham, K. Lu, J. Agudelo, R. Carrion, Jr., J. W. Nunneley, D. Barnard, S. Pohlmann, J. H. McKerrow, A. R. Renslo, and G. Simmons. 2015. Protease inhibitors targeting coronavirus and filovirus entry. Antiviral Res. 116:76-84.

76. Gierer, S., S. Bertram, F. Kaup, F. Wrensch, A. Heurich, A. Kramer-Kuhl, K. Welsch, M. Winkler, B. Meyer, C. Drosten, U. Dittmer, H. T. von, G. Simmons, H. Hofmann, and S. Pohlmann. 2013. The spike protein of the emerging betacoronavirus EMC uses a novel coronavirus receptor for entry, can be activated by TMPRSS2, and is targeted by neutralizing antibodies. J. Virol. 87:5502-5511.

77. Szabo, R. and T. H. Bugge. 2011. Membrane-anchored serine proteases in vertebrate cell and developmental biology. Annu. Rev. Cell Dev. Biol. 27:213-235.

78. Erikson, E., T. Adam, S. Schmidt, J. Lehmann-Koch, B. Over, C. Goffinet, C. Harter, I. Bekeredjian-Ding, S. Sertel, F. Lasitschka, and O. T. Keppler. 2011. In vivo expression profile of the antiviral restriction factor and tumor-targeting antigen CD317/BST-2/HM1.24/tetherin in humans. Proc. Natl. Acad. Sci. U. S. A 108:1368813693.

79. Swiecki, M., Y. Wang, S. Gilfillan, D. J. Lenschow, and M. Colonna. 2012. Cutting edge: paradoxical roles of BST2/tetherin in promoting type I IFN response and viral infection. J. Immunol. 188:2488-2492.

80. Stirnberg, M., E. Maurer, A. Horstmeyer, S. Kolp, S. Frank, T. Bald, K. Arenz, A. Janzer, K. Prager, P. Wunderlich, J. Walter, and M. Gutschow. 2010. Proteolytic processing of the serine protease matriptase-2: identification of the cleavage sites required for its autocatalytic release from the cell surface. Biochem. J. 430:87-95.

81. Chen, C. J., B. Y. Wu, P. I. Tsao, C. Y. Chen, M. H. Wu, Y. L. Chan, H. S. Lee, M. D. Johnson, R. L. Eckert, Y. W. Chen, F. Chou, J. K. Wang, and C. Y. Lin. 2011. Increased matriptase zymogen activation in inflammatory skin disorders. Am. J. Physiol Cell Physiol 300:C406-C415. 
82. Dreymueller, D., S. Uhlig, and A. Ludwig. 2015. ADAM-family metalloproteinases in lung inflammation: potential therapeutic targets. Am. J. Physiol Lung Cell Mol. Physiol 308:L325-L343.

83. Tavano, B., R. P. Galao, D. R. Graham, S. J. Neil, V. N. Aquino, D. Fuchs, and A. Boasso. 2013. Ig-like transcript 7, but not bone marrow stromal cell antigen 2 (also known as HM1.24, tetherin, or CD317), modulates plasmacytoid dendritic cell function in primary human blood leukocytes. J. Immunol. 190:2622-2630.

84. Kim, T. S., C. Heinlein, R. C. Hackman, and P. S. Nelson. 2006. Phenotypic analysis of mice lacking the Tmprss2-encoded protease. Mol. Cell Biol. 26:965-975. 


\section{FIGURE LEGENDS}

FIG 1 Type II transmembrane serine proteases antagonize tetherin. (A) 293T cells were cotransfected with HIV-1 p55-Gag plasmid, tetherin plasmid (right panel) or empty plasmid (left panel), and plasmids encoding either Vpu, EBOV-GP, the indicated protease or no protein (control). Expression of p55-Gag and $\beta$-actin in cell lysates and the release of p55Gag in culture supernatants were analyzed by Western blot. Each panel shows the result of a single Western blot from which irrelevant lanes were removed. Similar results were obtained in five separate experiments. (B) The experiment was carried out as described for (a) but catalytically inactive mutant of TMPRSS2 (TMPSS2 mut) was analyzed. The results were confirmed in two separate experiments. (C) To compare protease expression, 293T cells were transfected with plasmids encoding the indicated proteases equipped with a myc tag. Empty plasmid was used negative control (control) while a plasmid encoding myc-tagged EBOV VP40 was employed as positive control. Protein expression in cell lysates was detected by Western blot using anti-myc antibody. Detection of $\beta$-actin served as loading control. Similar results were obtained in two separate experiments. White filled arrowheads indicate zymogen forms while grey filled arrowheads highlight fragments generated upon activation and exhibiting a molecular weight expected for the protease domain. VLP, virus-like particle; TTSP, type II transmembrane serine protease; Sups, supernatants.

FIG 2 Interference of type II transmembrane serine proteases with tetherin expression. (A) 293T cells were cotransfected with a plasmid encoding tetherin and plasmids encoding the indicated TTSPs, Vpu or EBOV-GP or a plasmid encoding no protein (control). Expression of tetherin and $\beta$-actin in cell lysates was detected by Western blot. Similar results were obtained in three separate experiments. (B) The experiment was conducted as described for 
(A) but tetherin expression was detected by FACS. The average of two to five independent experiments is shown. Error bars indicate standard error of the mean (SEM). Tetherin expression on cells cotransfected with empty plasmid (control) and tetherin encoding plasmid was set as $100 \%$. Statistical significance was calculated using two-tailed students t-test.

FIG 3 Type II transmembrane serine proteases cleave tetherin. (A) 293T cells were transfected with plasmids encoding HIV-1 p55-Gag, tetherin and either EBOV-GP, Vpu or the indicated proteases or encoding no protein (control). Cells transfected with empty plasmid alone served as negative control. Expression of tetherin and $\beta$-actin in cell lysates and the presence of tetherin in culture supernatants were analyzed by Western blot. Similar results were obtained in three separate experiments. (B) The experiment was carried out as described for panel (A) but supernatants were treated with PNGase F before analysis by Western blotting. VLP, virus-like particle; Sups, supernatants.

FIG 4 TMPRSS2 and tetherin TMPRSS2 and tetherin colocalize and interact. (A) 293T cells were transfected to express the indicated CFP and YFP fusion proteins alone or in combination and subcellular localization was investigated by confocal spinning disc microscopy. The scale bar indicates a distance of $10 \mu \mathrm{m}$. (B) FACS-FRET analysis of $293 \mathrm{~T}$ cells transfected to express the indicated CFP and YFP fusion proteins. Mean values and SD were derived from 3 to 6 independent transfections. Statistical significance was calculated using two-tailed students t-test.

FIG 5 Endogenous expression of tetherin and TMPRSS2 determines the efficiency of HIV-1 release from Caco-2 cells. Caco-2 cells were cotransfected with plasmids encoding HIV-1 NL4-3 wt or HIV-1 NL4-3 $\Delta$ vpu and siRNAs targeting tetherin or TMPRSS2. Scrambled 
siRNA served as negative control (control). The expression of $\mathrm{p} 55-\mathrm{Gag}$, tetherin and $\beta$-actin expression in cell lysates and the release of p24-capsid into culture supernatants were analyzed by Western blot. Comparable results were obtained in two separate experiments. Sups, supernatants.

FIG 6 TMPRSS2 rescues release of authentic influenza virus from restriction by tetherin. (A) Caco- 2 cells were cotransfected with a plasmid encoding the NA of the 1918 influenza virus and siRNAs targeting tetherin or TMPRSS2. Scrambled siRNA served as negative control (control). The expression of NA, tetherin and $\beta$-actin in cell lysates and the release of NA into culture supernatants were analyzed by Western blot. Similar results were obtained in a separate experiment. Sups, supernatants. (B) 293T cells were transfected with plasmid encoding tetherin or transfected with empty plasmid (control) and infected with influenza viruses A/PR/8/34 (H1N1) and A/Panama/2007/99 (H3N2) at MOIs of 0.1 and 0.3, respectively. The cells were washed, cultured and at $24 \mathrm{~h}$ post infection the presence of infectious units in culture supernatants was determined by focus formation assay. The results of a representative experiment performed with sextuplicate samples are shown; errors bars indicate standard deviation (SD). The results were confirmed in two separate experiments. (C) The experiment was carried out as described for panel (B) but target cells were transfected with plasmids encoding tetherin wt or tetherin lacking the transmembrane domain $(\Delta \mathrm{TM})$ and subsequently infected with $\mathrm{A} / \mathrm{PR} / 8 / 34(\mathrm{H} 1 \mathrm{~N} 1)$. The results of a representative experiment performed with sextuplicate samples are shown; errors bars indicate SD. Similar results were obtained in a separate experiment. (D) The experiment was carried out as described for panel (C) but 293 T cells were transfected with plasmids encoding TMPRSS2 wt or enzymatically inactive TMPRSS2 (TMPRSS2 mut) in the presence and absence of tetherin expression plasmid followed by infection with A/PR/8/34 (H1N1). The presence of 
infectious units in culture supernatants was determined at $8 \mathrm{~h}$ post infection. The results of a representative experiment performed with sextuplicate samples are shown; errors bars indicate SD. A separate experiment yielded comparable results. (E) Caco2 cells were transfected with siRNA specific for tetherin or TMPRSS2 or transfected with scrambled, non-sense siRNA (control) and subsequently infected with A/PR/8/34 at an MOI of 0.01 . Virus titers in culture supernatants were determined at $24 \mathrm{~h}$ post infection. The results of a single representative experiment performed with triplicate samples are shown and were confirmed in three separate experiments. Error bars indicate SD. Statistical significance was calculated using two-tailed student's t-test.

FIG 7 Tetherin and TMPRSS2 are coexpressed in human bronchial cells. Human bronchial tissue segments were stained with antibodies directed against TMPRSS2 (red), Tetherin (green), $\beta$-Tubulin (ciliated cells; magenta) and with DAPI (cell nucleus; cyan) to determine the tetherin distribution. The result of a representative confocal image of 5 random selected fields is shown. Bar, $20 \mu \mathrm{m}$. 
Figure 1

Krämer-Kühl et al.

A)

Tetherin

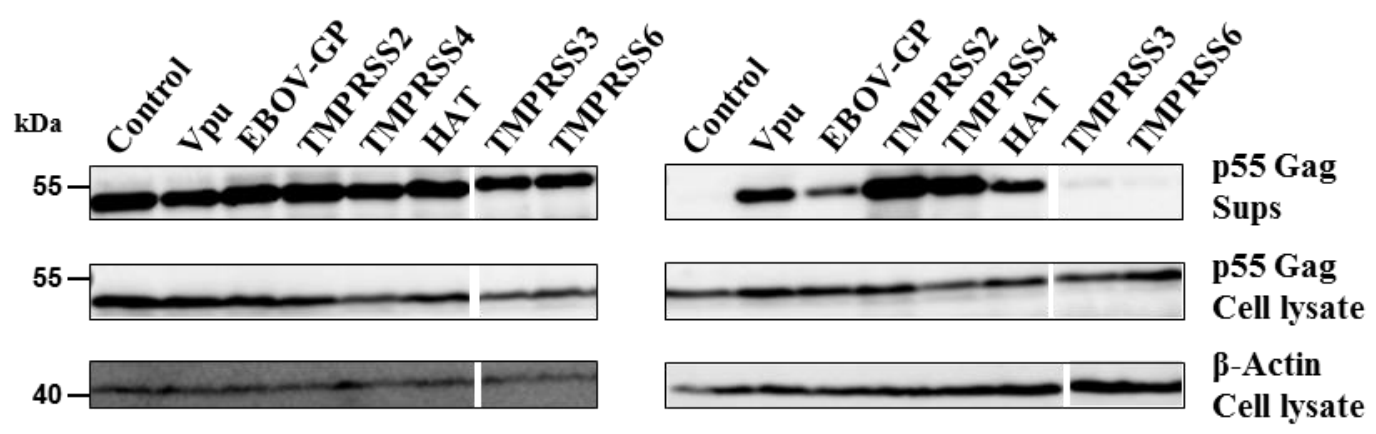

B)

C)
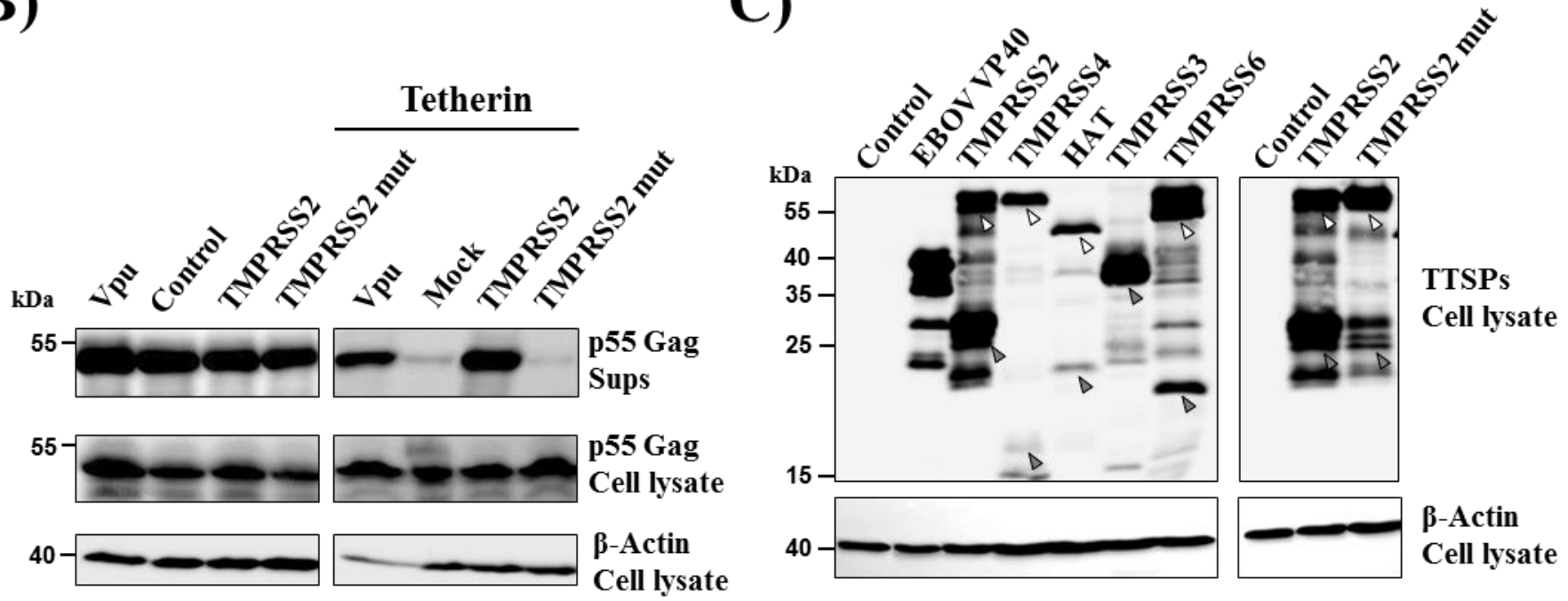
Figure 2

\section{Krämer-Kühl et al.}

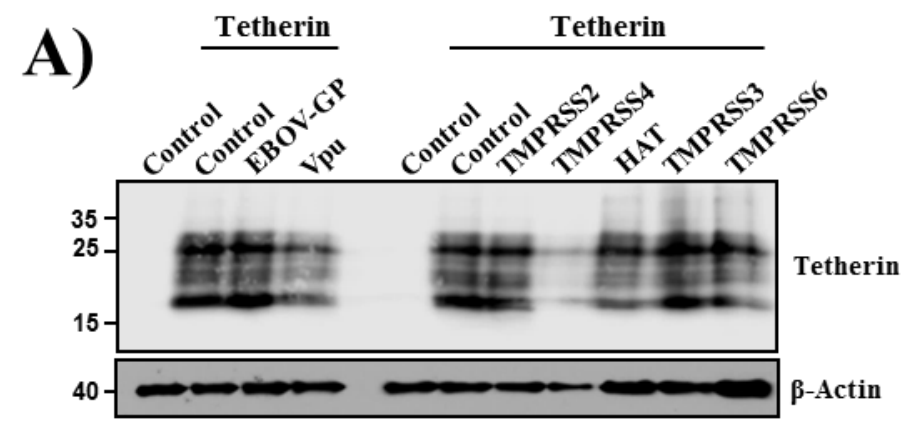

B)

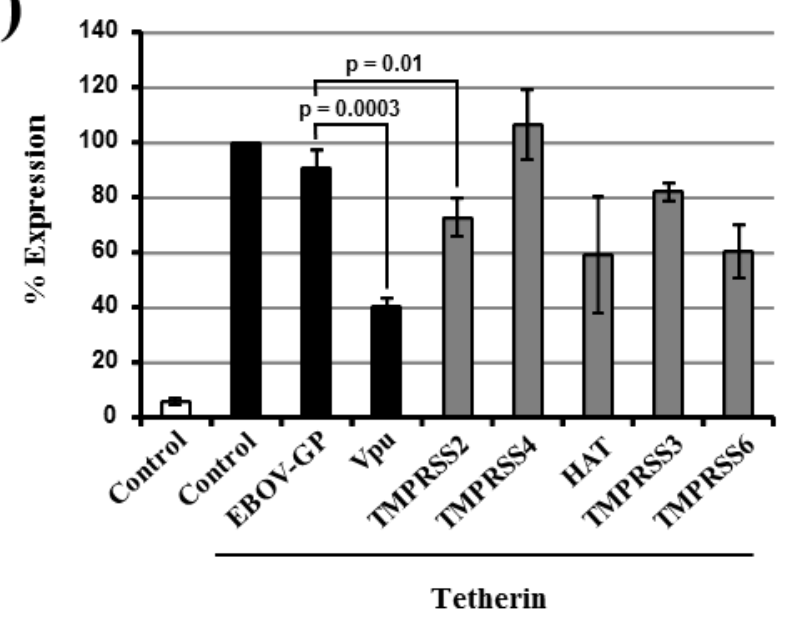


Figure 3

Krämer-Kühl et al.

A)

Tetherin
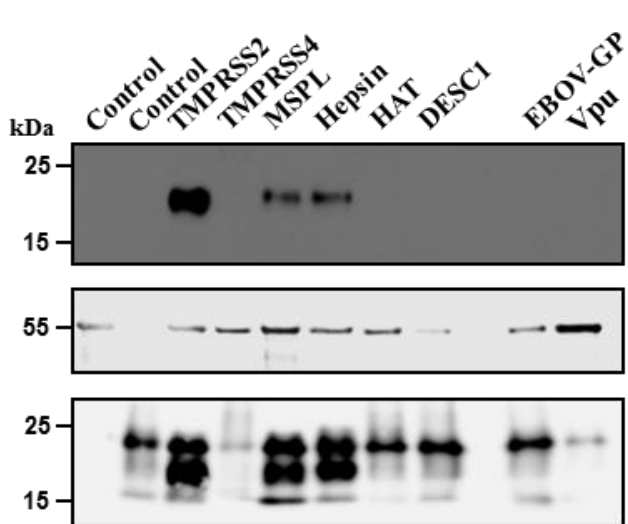

$55-\longrightarrow-\infty-\infty$

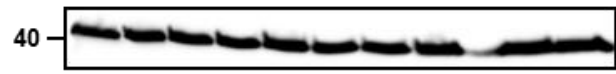

B)

Tetherin Tetherin + PNGase F

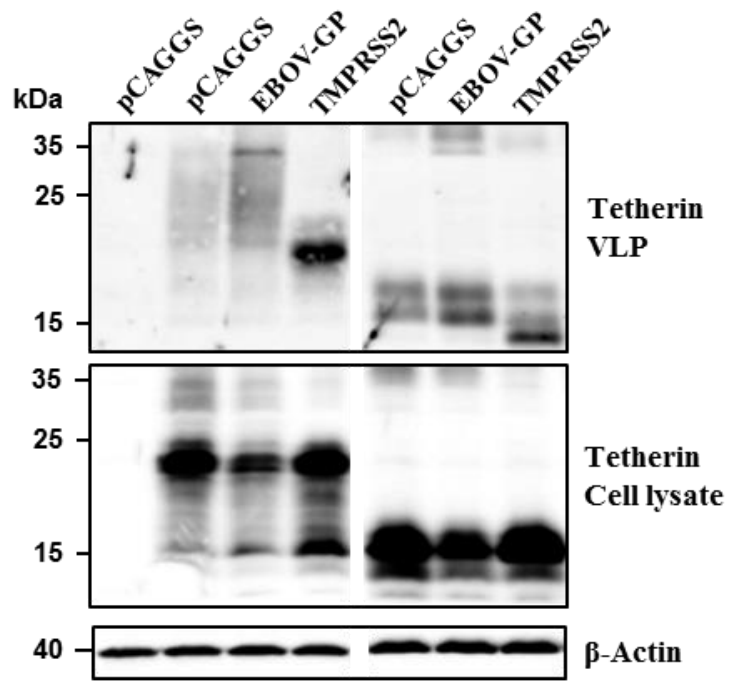

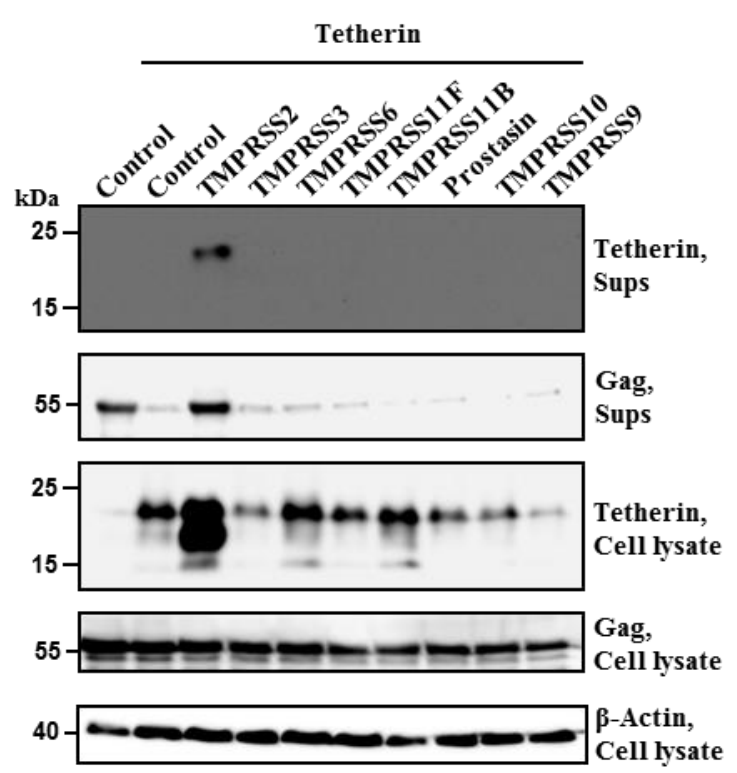


Figure 4

Krämer-Kühl et al.

A)

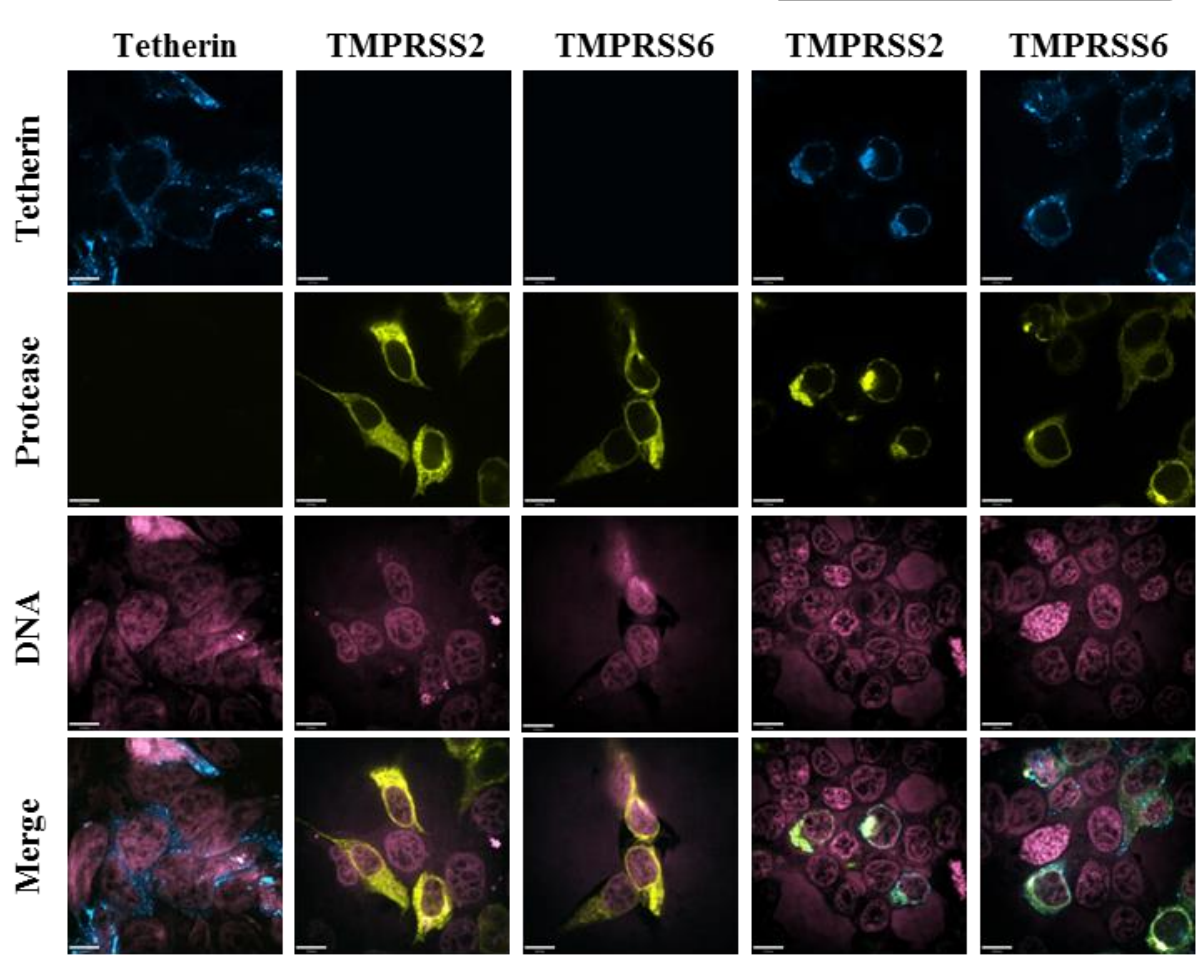

B)

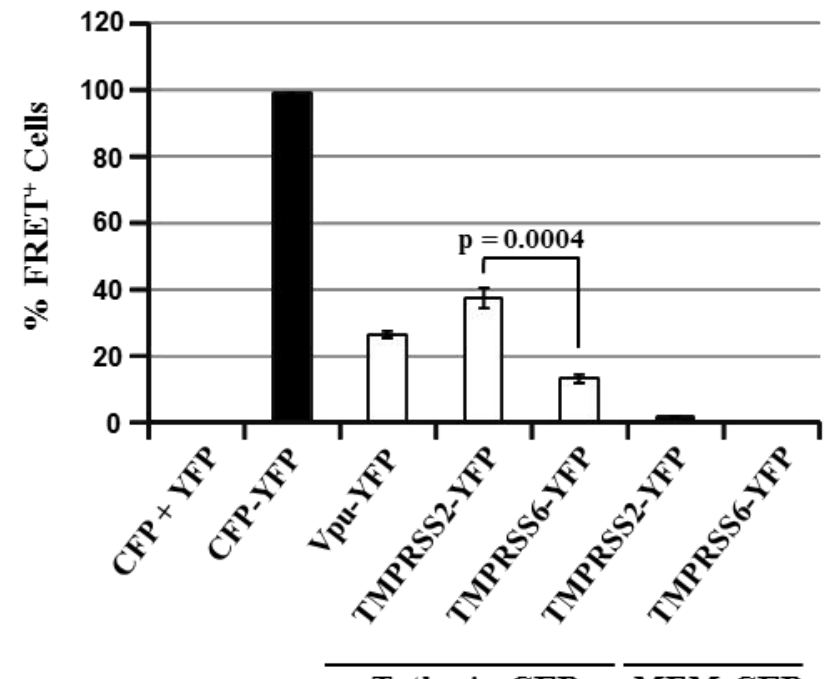

Tetherin-CFP $\overline{\text { MEM-CFP }}$ 
Figure 5

Krämer-Kühl et al.

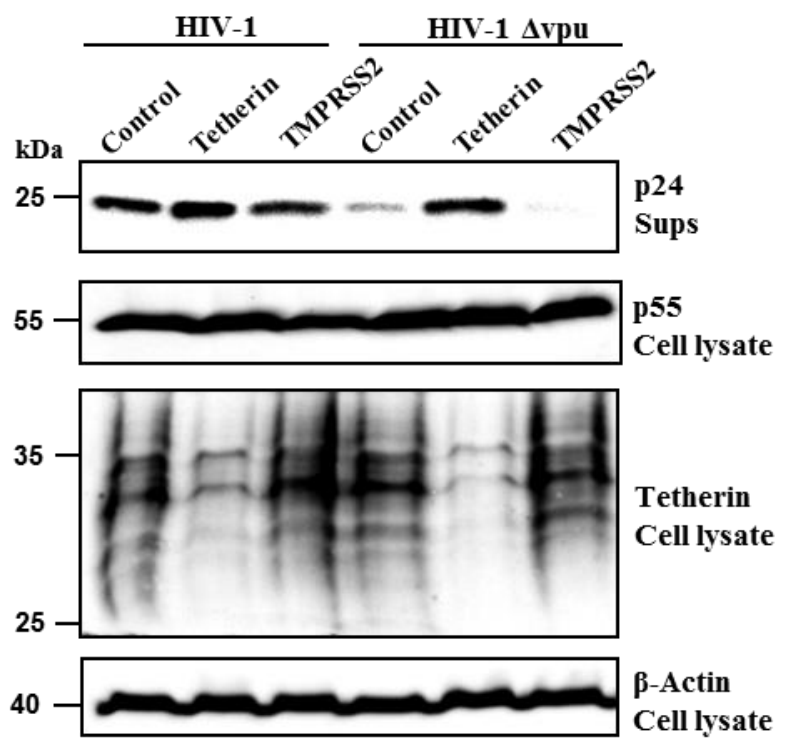


Figure 6

A)

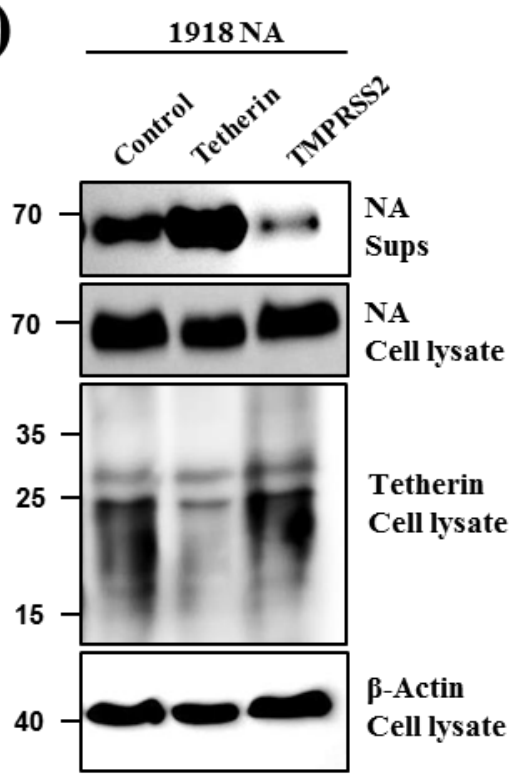

D)

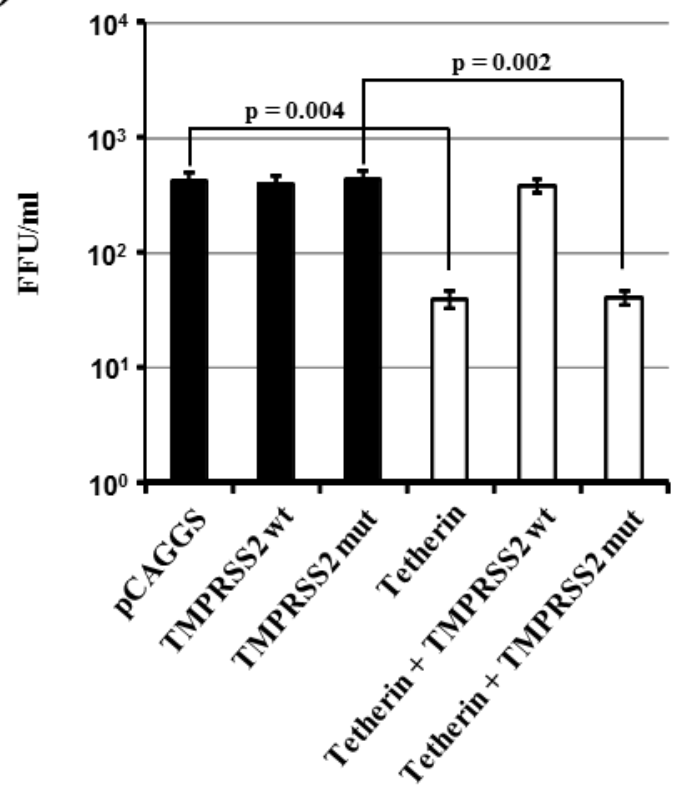

Krämer-Kühl et al.

B)

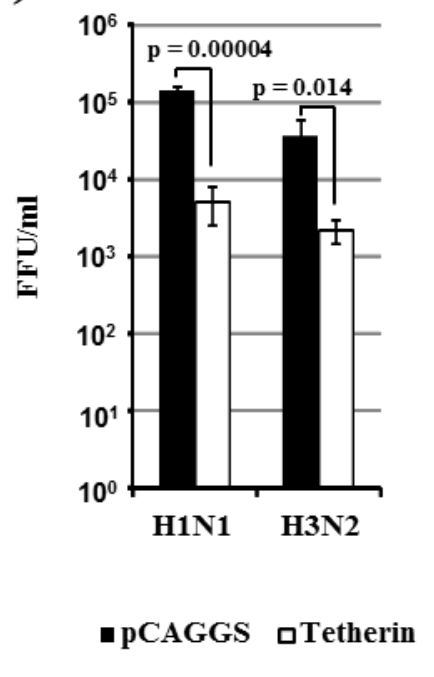

C)

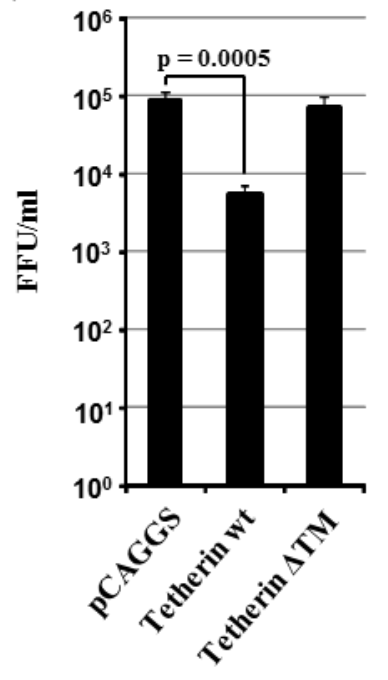

E)

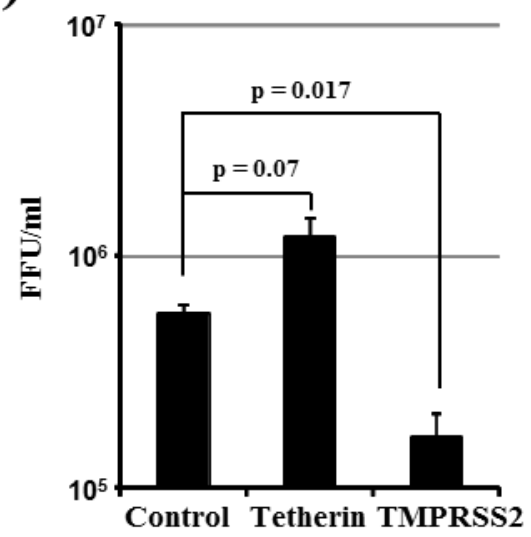


Figure 7

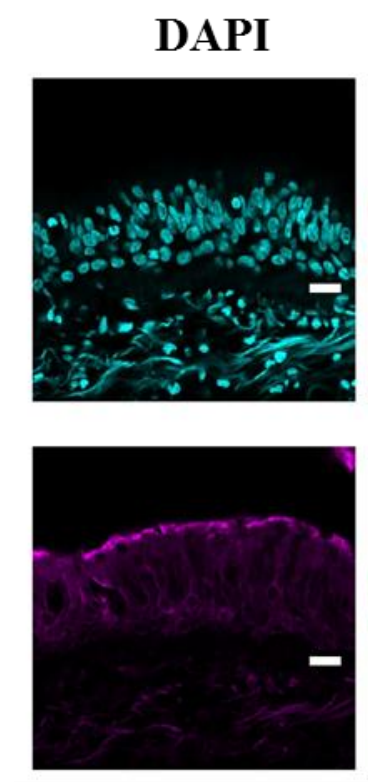

$\beta$-Tubulin
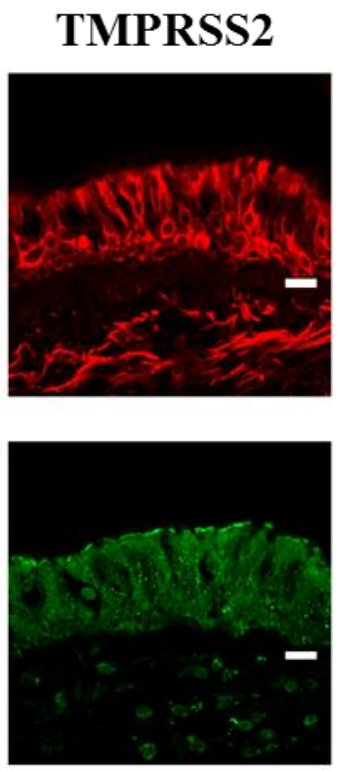

Tetherin
Krämer-Kühl et al.

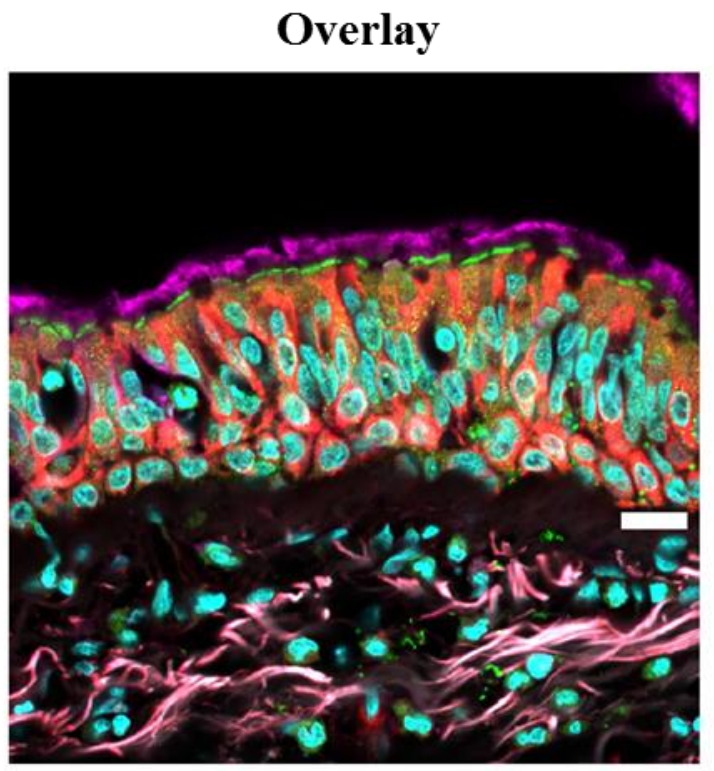




\section{Discussion}

\subsection{Novel activators of influenza virus hemagglutinin}

The human type II transmembrane serine protease family contains 19 members, which are expressed in the respiratory tract, the target for influenza virus, and other tissues (Antalis et al., 2011). At the beginning of this study, it was known that TMPRSS2, HAT, TMPRSS4 and matriptase can cleave and activate HA, at least upon directed expression (Baron et al., 2013; Beaulieu et al., 2013; Böttcher et al., 2006; Chaipan et al., 2009; Hamilton et al., 2012a), while hepsin was found to cleave but not activate FLUAV HA (Bertram et al., 2010a). In contrast, TMPRSS3, TMPRSS5 and TMPRSS6 were reported to be unable to process HA (Bertram et al., 2010a). The ability of the remaining 12 TTSPs to activate HA was unknown. The results reported in manuscript 1 'DESC1 and MSPL activate influenza A viruses and emerging coronaviruses for host cell entry', Zmora et al., J Virol, 2014, demonstrate that the TTSPs DESC1 and MSPL cleave HA and that cleavage results in HA activation.

DESC1 (differentially expressed in squamous cell carcinoma 1) is expressed in prostate, skin, testis, head, neck, kidney and esophagus, while expression is downregulated in some squamous cell carcinomas (Hobson et al., 2004; Lang and Schuller, 2001; Sedghizadeh et al., 2006; Uhlen et al., 2015). The present study demonstrates that a small amount of Descl mRNA is expressed in human lung, confirming observations reported by Sales et al. (Sales et al., 2011). Additionally, immunohistochemistry revealed that DESC1 protein is present in the plasma membrane of cells in renal glomeruli and in squamous epithelium of the esophagus and the uterine cervix (Uhlen et al., 2015). The physiological function of DESC1 is not known (Antalis et al., 2011). Proteomic Identification of protease Cleavage Sites (PICS) which analyses proteome-derived peptide libraries showed that DESC1 prefers large hydrophobic residues in P4/P3, small residues in P2, Arg or Lys in P1 and hydrophobic residues in P1' and P3' (Barre et al., 2014) and this preference is compatible with the cleavage motif in the influenza virus HA.

MSPL (mosaic serine protease long-form) is expressed at low level in the lung, placenta and pancreas, and normal prostate shows strong cytoplasmic staining (Guipponi et al., 2008b; Kido and Okumura, 2008; Uhlen et al., 2015). The present study confirmed that $M s p l$ mRNA is expressed in the human lung but at lower levels compared to Tmprss2 
transcripts. This enzyme preferentially recognizes cleavage sites consisting of paired basic amino acid residues, i.e. arginine or lysine, and is strongly inhibited by serine protease inhibitors like aprotinin, banzamidine and Bowman-Birk trypsin inhibitor, but shows some resistance to $\alpha$-1-antitrypsin and leupeptin (Kido and Okumura, 2008). Recently, it was demonstrated that $M s p l$-deficient mice displayed abnormal skin development which resulted in a compromised barrier function (Madsen et al., 2014). Thus, MSPL seems to contribute to stratum corneum formation and epidermal barrier acquisition (Madsen et al., 2014). Additionally, it was observed that MSPL supports avian influenza virus replication by proteolytic activation of HA (Okumura et al., 2010), which confirms and extends the observations reported in manuscript 1 .

The observation that absence of TMPRSS2 expression protects mice only partially against H3N2 infection and pathogenesis suggests that some FLUAV might be able to proteases other than TMPRSS2 for spread in the host and members of the TTSP family are interesting candidates (Sakai et al., 2015). However, assessing the contribution of DESC1 and MSPL to FLUAV spread in the host will be challenging. Thus, there are no cell lines described to express endogenous DESC1 or MSPL at the protein level precluding experiments assessing whether these proteases can activate FLUAV upon endogenous expression. Moreover, there are no Descl-deficient mice, which could be employed to analyze the contribution of this protease to viral spread in vivo. $M s p l$-deficient mice were reported (Madsen et al., 2014) and were found to display aberrant skin development (Madsen et al., 2014). Therefore, it might be difficult to discern direct antiviral effects associated with loss of MSPL expression from indirect effects due to tissue malformation.

In Manuscript 1, TMPRSS11F, prostasin, TMPRSS11B, TMPRSS10 and TMPRSS9 were found to be unable to activate HA. All proteins were expressed in transiently transfected $293 \mathrm{~T}$ cells, although significant differences in expression levels were found. Therefore, it cannot be excluded that absence of HA activation by the above mentioned proteases was due to insufficient expression. In contrast, failure of HA cleavage was not due to lack of autocatalytic activation, since products of autocatalytic activation were detected for all proteases investigated. Despite the fact that TMPRSS11F, prostasin and TMPRSS11B were cloned from the human lung mRNA during the studies described in manuscript 1 , the expression of these enzymes in other organs is more prominent. Thus, TMPRSS11F and TMPRSS11B are mostly expressed in the gastrointestinal tract, while prostasin can be found in skeletal muscles and testis (Uhlen et al., 2015). Therefore, it cannot be excluded that the 
activity of these three proteases is restricted to different organs, and thus the HA is not processed by them.

Three TTSPs, enteropeptidase, matriptase- 3 and TMPRSS11A, were not examined in manuscript 1 due to low or absent expression in the human lung (Uhlen et al., 2015). Thus, these enzymes remain to be tested for HA activation and these experiments are ongoing. TMPRSS11A is an interesting candidate for HA activation, since this enzyme was shown to cleave recombinant native trimers of the $S$ protein of SARS-CoV (Kam et al., 2009). The $\mathrm{S}$ protein of SARS-CoV and MERS-CoV can be activated by both the endosomal cysteine proteases cathepsin B/L and TMPRSS2 as well other TTSPs (Simmons et al., 2013), and serine protease activity was shown to be essential for viral spread in a rodent model (Zhou et al., 2015). Moreover, TMPRSS11a is expressed in the upper respiratory tract (Uhlen et al., 2015) and could thus support viral spread in the host. The question why some TTSPs (e.g. TMPRSS2, MSPL, HAT and DESC1) can cleave HA, while others cannot (e.g. TMPRSS3, TMPRSS11F, prostasin), remains open and will be discussed below.

\subsection{Determinants of HA cleavage}

The observation that TMPRSS2 and other TTSPs can active HA in cell culture (Baron et al., 2013; Beaulieu et al., 2013; Böttcher et al., 2006; Chaipan et al., 2009; Hamilton et al., 2012a) raised the question why other members of the TTSP family fail to do so, despite efficient expression in transfected cells. Manuscript 2 'The stem domain of type II transmembrane serine proteases determines the activation of viral glycoproteins and is a potential target for intervention' Zmora et al., J Virol submitted, reports efforts to identify which domain(s) in TTSPs control the ability to activate HA. For this purpose, chimeras between TMPRSS2, which was previously demonstrated to cleave and activate HA (Böttcher et al., 2006), and TMPRSS3, a closely related protease which fails to activate HA (Bertram et al., 2010a), were constructed. The exchange of the cytoplasmic domain or the cytoplasmic jointly with the transmembrane domain did not alter HA activation (or lack thereof). In contrast, replacement of the TMPRSS2 cytoplasmic, transmembrane and stem region by that of TMPRSS3 (chimera 3-3-3-2) abrogated HA activation while the reverse changed endowed TMPRSS3 with the ability to cleave and activate HA (chimera 2-2-2-3). In addition, the replacement of the TMPRSS3 stem region by that of TMPRSS2 conferred HA activation to the chimeric enzyme and a similar observation was made with TMPRSS3 (inactive) 
harboring the stem region of TMPRSS4 (active). Thus, the stem region is the central determinant of HA activation.

A role of the stem region in the recognition and processing of physiological substrates was also demonstrated by others. Knappe and colleagues found that frizzled 1 domain and LDLR repeats $1-4$, the structural elements of stem region of corin, are required for processing of the substrate pro-ANP (Knappe et al., 2004). By construction of soluble corin consisted only of the catalytic domain, it was shown that the stem region is not required for processing of small peptide substrates, but is essential for pro-ANP cleavage. These authors suggested that stem region is responsible for substrate specificity, because it is involved in the direct interaction between corin and pro-ANP (Knappe et al., 2004). Manuscript 2 indicates that the stem region might impact substrate specificity by controlling the cellular localization of TTSPs, as discussed below.

One of the major determinants of a protein's function is its subcellular localization and the presence of enzyme and substrate in the same location within the cell is required for efficient catalysis (van Albada and ten Wolde, 2007). In manuscript 1 it was demonstrated that the HA-activating TTSPs TMPRSS2, DESC1 and MSPL colocalize with HA at or near the plasma membrane of infected cells, resulting in a Pearson Correlation Coefficient (PCC, a measure of colocalization) above 0.7. In contrast, TMPRSS3, which cannot activate HA, failed to colocalize with this protein, which is reflected by a PCC below 0.1 . It needs to be considered that the cellular localization of TTSPs might be dependent on the cell type and the expression level, which might have been unnaturally high in the COS-7 cells used in manuscript 1, since these cells were transiently transfected to express TTSPs. Nevertheless, the findings described above suggest that colocalization of TTSPs with HA is required for HA activation and raised the question whether the stem region determines the cellular localization of TTSPs. Indeed, the HA-activating chimera (2-2-2-3) was present at the plasma membrane and colocalized with HA, while the chimera which failed to activate HA (3-3-3-2) also failed to colocalize with HA. These findings suggest that the stem region can determine HA activation by controlling the cellular localization of TTSPs. A role of the stem region in the cellular trafficking of TTSPs was also suggested in reviews (Antalis et al., 2011; Szabo and Bugge, 2011) but data supporting these claims are largely missing.

The key role of the stem region in HA activation triggered interest in determining whether the stem region can be a target for inhibitors of TTSP activity. Members of the 
Hepsin/TMPRSS subfamily contain a group A scavenger receptor domain in the stem region, which in the context of scavenger receptor type A (SR-A) is responsible for binding of lowdensity lipoproteins and for removal of these proteins from the circulation (Canton et al., 2013). Dextran sulphate is an inhibitor of SR-A (Tsubamoto et al., 1994). Therefore, it was tested whether dextran sulphate inhibits HA activation by TTSPs contain an SR domain in their stem region. Activation of FLUAV by all TTSPs containing a SR domain was significantly inhibited by dextran sulphate, while virus activation by TTSPs not harboring the SR domain was not affected. The ability of dextran sulphate to suppress FLUAV spread correlated with inhibition of HA cleavage in transfected cells, although the effects observed were minor, likely due to high expression of protease and HA in the experimental system used. Collectively, these data suggest that targeting the stem region might a suitable way to prevent FLUAV activation by TTSPs. However, future studies must provide direct evidence that dextran sulphate bound to the SR domain and that binding was required for inhibition of HA activation. Additional proof of concept could be provided by raising antibodies against the SR domain of TTSPs and by testing their ability to block HA activation.

Inhibition of FLUAV activation by dextran sulphate was specific to TTSPs containing an SR domain but demonstrates that blockade of these enzymes could be a powerful tool to suppress FLUAV spread. This notion is in keeping with several findings indicating that broad spectrum protease inhibitors can suppress FLUAV spread in cell culture and in the host (Zhirnov et al., 2011). Recently, it was found that also endogenous protease inhibitors can block FLUAV spread. Dittmann et al. demonstrated that plasminogen activator inhibitor 1, the product of serpinel gene, can inhibit the proteolytic processing of HA by TMPRSS2 and HAT (Dittmann et al., 2015). Additionally, it was observed that FLUAV infection in serpine1-/- mice was more severe and lethal (Dittmann et al., 2015). Moreover, the hepatocyte growth activator inhibitor 2 (HAI2) was found to inhibit HA activation in cell culture (Hamilton et al., 2014). Whether the inhibitory effect of these inhibitors extends to all HA activating TTSPs is unknown.

\subsection{Different TMPRSS2 isoforms activate HA}

The transcripts encoding TTSPs can be alternatively spliced and this may have important consequences for enzymatic activity. Qi et al. demonstrated that the alternative splicing of the corin transcript results in the production of two isoforms, E1 and E1a, which differ in the presence of an N-terminal extension of 30 amino acids, which alters cellular 
localization and enzymatic activity (Qi et al., 2011). The Tmprss 2 transcript can also be alternatively spliced and this results in the generation of isoforms 1 and 2 which differ only in the N-terminal cytoplasmic tail (Jacquinet et al., 2001; Paoloni-Giacobino et al., 1997). The Tmprss2 transcript variant 1 (encoding TMPRSS2 isoform 1) contains additional 111 nucleotides, which encode a 37 amino acids comprising $\mathrm{N}$-terminal extension in comparison to transcript variant 2 (encoding for TMPRSS2 isoform 2), posing the question whether this extension impacts HA activation. The study reported in manuscript 4 'TMPRSS2 isoforms 1 activates respiratory viruses and is expressed in viral target cells' Zmora et al., PLOS ONE, 2015, shows that TMPRSS2 isoforms 1 and 2 are comparably expressed in transfected cells and demonstrates that potential FLUAV target cells in the human lung express transcript variant 1 . Moreover, the results show that both isoforms cleave and activate FLUAV and colocalize with HA at or near to the plasma membrane. Moreover, the analysis of the $\mathrm{N}$-terminal sequence of TMPRSS2 isoform 1 with MmM 3.0 (Minimotif Miner), MotifScan and CombFunc Protein Function Prediction Server, Imperial College London, did not detect motifs characteristic for endocytosis, intracellular signaling or localization. Therefore, the cytoplasmic tail of TMPRSS2 might not impact HA cleavage. However, the analysis of HA cleavage and colocalization of isoforms 1 and 2 was carried out with transfected cells and it can at present not be excluded that potential differences in expression and function of the isoforms were obscured by high expression of these enzymes in the transfected cells.

\subsection{The role of TTSPs in influenza virus release}

Many published studies, including the work discussed above, demonstrate that TTSPs can activate viral glycoproteins and thereby play an important role in viral entry (Bertram et al., 2010b; Garten et al., 2015). Notably, TTSPs may also contribute to viral release. Shirato and colleagues found that PEDV release was promoted by expression of TMPRSS2 while intracellular replication was not affect (Shirato et al., 2011). Thus, large clusters of virions were retained at the surface of infected cells which failed to express TMPRSS2 or were treated with a serine protease inhibitor, while these structures were not observed when TMPRSS2 was expressed (Shirato et al., 2011). These results suggest that TMPRSS2 might counteract a cellular factor which interferes with viral release. The IFN-induced host cell protein tetherin inhibits release of several enveloped viruses from infected cells (in the absence of a virus-encoded tetherin antagonist) and is an important component of the innate defenses against viral infection (Neil et al., 2008; Van Damme et al., 2008). Whether FLUAV can be inhibited by tetherin was unclear. Some studies demonstrated that FLUAV are tetherin 
insensitive (Bruce et al., 2012; Watanabe et al., 2011; Winkler et al., 2012), while other studies demonstrated tetherin-mediated inhibition of viral release (Leyva-Grado et al., 2014; Mangeat et al., 2012). One possibility to account for these discrepant observations is that FLUAV inhibition by tetherin might be dependent on the viral strain examined. Therefore, it was first tested whether laboratory-adapted, seasonal and pandemic FLUAV differ in tetherin sensitivity.

Manuscript 4 'Tetherin sensitivity of influenza A viruses is strain specific: Role of hemagglutinin and neuraminidases', Gnirß, Zmora et al., J Virol, 2015, shows that laboratory-adapted and seasonal FLUAV strains are sensitive to inhibition by tetherin while pandemic strains are resistant, and resistance was linked to the HA and NA proteins. Importantly, these results were confirmed in different experimental systems, including cells which exogenously or endogenously express tetherin. However, the mechanism underlying tetherin antagonism by HA and NA remained largely unclear, although evidence was obtained that tetherin-resistant FLUAV reduce intracellular expression of tetherin (manuscript 4). Whether this effect is due to HA/NA induced tetherin degradation and whether degradation occurs in endo-/lysosomes or in proteasomes remains to be established. Moreover, it will be important to examine a broader panel of FLUAV strains for tetherin sensitivity, including strains responsible for all FLUAV pandemics since 1918 as well as LPAIV and HPAIV. In sum, the results reported in manuscript 4 show that certain FLUAV are tetherin-sensitive and raised the question whether TTSP expression rescues these strains from inhibition by tetherin.

Manuscript 5 'Influenza virus activating host cell proteases antagonize tetherin' Krämer-Kühl, Zmora, Blazejewska and Gnirß et al., J Virol, submitted, demonstrates that TTSPs, which cleave and activate HA, TMPRSS2, TMPRSS4, HAT, DESC1 and MSPL, also cleave tetherin and rescue FLUAV from tetherin-mediated inhibition. These findings suggest that infection of TTSP-positive cells might be associated with two benefits for FLUAV, HA activation and tetherin antagonism. A central question which remains to be answered is whether tetherin antagonism by TTSPs is operative in vivo. Two observations suggest that this might be the case: First, siRNA-mediated knock-down of tetherin expression in Caco-2 cells increased FLUAV spread while knock-down of TMPRSS2 expression had the opposite effect. Second, tetherin and TMPRSS2 were coexpressed in human respiratory epithelium and thus in potential FLUAV target cells. However, increase of viral spread in Caco-2 cells upon tetherin knock-down was modest and ultimately animal studies will be 
required to assess the in vivo relevance of TTSP-mediated tetherin antagonism. The major challenge associated with these studies it to discriminate TTSP-mediated augmentation of FLUAV spread due to HA activation and tetherin antagonism. 


\title{
6. List of references
}

\author{
http://www.cdc.gov/flu/.
}

www.who.int.

Abe, M., Tahara, M., Sakai, K., Yamaguchi, H., Kanou, K., Shirato, K., Kawase, M., Noda, M., Kimura, H., Matsuyama, S., Fukuhara, H., Mizuta, K., Maenaka, K., Ami, Y., Esumi, M., Kato, A., Takeda, M., 2013. TMPRSS2 is an activating protease for respiratory parainfluenza viruses. J Virol 87, 11930-11935.

Afar, D.E., Vivanco, I., Hubert, R.S., Kuo, J., Chen, E., Saffran, D.C., Raitano, A.B., Jakobovits, A., 2001. Catalytic cleavage of the androgen-regulated TMPRSS2 protease results in its secretion by prostate and prostate cancer epithelia. Cancer Res 61, 1686-1692.

Ali, A., Avalos, R.T., Ponimaskin, E., Nayak, D.P., 2000. Influenza virus assembly: effect of influenza virus glycoproteins on the membrane association of M1 protein. J Virol 74, 87098719.

Antalis, T.M., Bugge, T.H., Wu, Q., 2011. Membrane-anchored serine proteases in health and disease. Prog Mol Biol Transl Sci 99, 1-50.

Baek, Y.H., Song, M.S., Lee, E.Y., Kim, Y.I., Kim, E.H., Park, S.J., Park, K.J., Kwon, H.I., Pascua, P.N., Lim, G.J., Kim, S., Yoon, S.W., Kim, M.H., Webby, R.J., Choi, Y.K., 2015. Profiling and characterization of influenza virus N1 strains potentially resistant to multiple neuraminidase inhibitors. J Virol 89, 287-299.

Baranovich, T., Bahl, J., Marathe, B.M., Culhane, M., Stigger-Rosser, E., Darnell, D., Kaplan, B.S., Lowe, J.F., Webby, R.J., Govorkova, E.A., 2015. Influenza A viruses of swine circulating in the United States during 2009-2014 are susceptible to neuraminidase inhibitors but show lineage-dependent resistance to adamantanes. Antiviral Res 117, 10-19.

Baron, J., Tarnow, C., Mayoli-Nussle, D., Schilling, E., Meyer, D., Hammami, M., Schwalm, F., Steinmetzer, T., Guan, Y., Garten, W., Klenk, H.D., Bottcher-Friebertshauser, E., 2013. Matriptase, HAT, and TMPRSS2 activate the hemagglutinin of H9N2 influenza A viruses. J Virol 87, 1811-1820.

Barre, O., Dufour, A., Eckhard, U., Kappelhoff, R., Beliveau, F., Leduc, R., Overall, C.M., 2014. Cleavage specificity analysis of six type II transmembrane serine proteases (TTSPs) using PICS with proteome-derived peptide libraries. PLoS One 9, e105984.

Beaulieu, A., Gravel, E., Cloutier, A., Marois, I., Colombo, E., Desilets, A., Verreault, C., Leduc, R., Marsault, E., Richter, M.V., 2013. Matriptase proteolytically activates influenza virus and promotes multicycle replication in the human airway epithelium. J Virol 87, 4237 4251.

Benaud, C.M., Oberst, M., Dickson, R.B., Lin, C.Y., 2002. Deregulated activation of matriptase in breast cancer cells. Clin Exp Metastasis 19, 639-649.

Bertram, S., Dijkman, R., Habjan, M., Heurich, A., Gierer, S., Glowacka, I., Welsch, K., Winkler, M., Schneider, H., Hofmann-Winkler, H., Thiel, V., Pöhlmann, S., 2013. TMPRSS2 activates the human coronavirus $229 \mathrm{E}$ for cathepsin-independent host cell entry and is expressed in viral target cells in the respiratory epithelium. J Virol 87, 6150-6160.

Bertram, S., Glowacka, I., Blazejewska, P., Soilleux, E., Allen, P., Danisch, S., Steffen, I., Choi, S.Y., Park, Y., Schneider, H., Schughart, K., Pöhlmann, S., 2010a. TMPRSS2 and 
TMPRSS4 facilitate trypsin-independent spread of influenza virus in Caco-2 cells. J Virol 84, 10016-10025.

Bertram, S., Glowacka, I., Müller, M.A., Lavender, H., Gnirss, K., Nehlmeier, I., Niemeyer, D., He, Y., Simmons, G., Drosten, C., Soilleux, E.J., Jahn, O., Steffen, I., Pöhlmann, S., 2011. Cleavage and activation of the severe acute respiratory syndrome coronavirus spike protein by human airway trypsin-like protease. J Virol 85, 13363-13372.

Bertram, S., Glowacka, I., Steffen, I., Kühl, A., Pöhlmann, S., 2010b. Novel insights into proteolytic cleavage of influenza virus hemagglutinin. Rev Med Virol 20, 298-310.

Bertram, S., Heurich, A., Lavender, H., Gierer, S., Danisch, S., Perin, P., Lucas, J.M., Nelson, P.S., Pöhlmann, S., Soilleux, E.J., 2012. Influenza and SARS-coronavirus activating proteases TMPRSS2 and HAT are expressed at multiple sites in human respiratory and gastrointestinal tracts. PLoS One 7, e35876.

Biere, B., Bauer, B., Schweiger, B., 2010. Differentiation of influenza B virus lineages Yamagata and Victoria by real-time PCR. Journal of clinical microbiology 48, 1425-1427.

Böttcher-Friebertshäuser, E., Freuer, C., Sielaff, F., Schmidt, S., Eickmann, M., Uhlendorff, J., Steinmetzer, T., Klenk, H.D., Garten, W., 2010. Cleavage of influenza virus hemagglutinin by airway proteases TMPRSS2 and HAT differs in subcellular localization and susceptibility to protease inhibitors. J Virol 84, 5605-5614.

Bottcher-Friebertshauser, E., Klenk, H.D., Garten, W., 2013. Activation of influenza viruses by proteases from host cells and bacteria in the human airway epithelium. Pathog Dis 69, 87100.

Böttcher-Friebertshäuser, E., Stein, D.A., Klenk, H.D., Garten, W., 2011. Inhibition of influenza virus infection in human airway cell cultures by an antisense peptide-conjugated morpholino oligomer targeting the hemagglutinin-activating protease TMPRSS2. J Virol 85, 1554-1562.

Böttcher, E., Matrosovich, T., Beyerle, M., Klenk, H.D., Garten, W., Matrosovich, M., 2006. Proteolytic activation of influenza viruses by serine proteases TMPRSS2 and HAT from human airway epithelium. J Virol 80, 9896-9898.

Bruce, E.A., Abbink, T.E., Wise, H.M., Rollason, R., Galao, R.P., Banting, G., Neil, S.J., Digard, P., 2012. Release of filamentous and spherical influenza A virus is not restricted by tetherin. The Journal of general virology 93, 963-969.

Bugge, T.H., Antalis, T.M., Wu, Q., 2009. Type II transmembrane serine proteases. J Biol Chem 284, 23177-23181.

Cal, S., Quesada, V., Garabaya, C., Lopez-Otin, C., 2003. Polyserase-I, a human polyprotease with the ability to generate independent serine protease domains from a single translation product. Proc Natl Acad Sci U S A 100, 9185-9190.

Canton, J., Neculai, D., Grinstein, S., 2013. Scavenger receptors in homeostasis and immunity. Nature reviews. Immunology 13, 621-634.

Chaipan, C., Kobasa, D., Bertram, S., Glowacka, I., Steffen, I., Tsegaye, T.S., Takeda, M., Bugge, T.H., Kim, S., Park, Y., Marzi, A., Pöhlmann, S., 2009. Proteolytic activation of the 1918 influenza virus hemagglutinin. J Virol 83, 3200-3211.

Chakrabarti, A.K., Pasricha, G., 2013. An insight into the PB1F2 protein and its multifunctional role in enhancing the pathogenicity of the influenza A viruses. Virology 440, 97-104. 
Chen, B.J., Takeda, M., Lamb, R.A., 2005. Influenza virus hemagglutinin (H3 subtype) requires palmitoylation of its cytoplasmic tail for assembly: M1 proteins of two subtypes differ in their ability to support assembly. J Virol 79, 13673-13684.

Chen, J., Lee, K.H., Steinhauer, D.A., Stevens, D.J., Skehel, J.J., Wiley, D.C., 1998. Structure of the hemagglutinin precursor cleavage site, a determinant of influenza pathogenicity and the origin of the labile conformation. Cell 95, 409-417.

Chen, S., Sen, S., Young, D., Wang, W., Moravec, C.S., Wu, Q., 2010. Protease corin expression and activity in failing hearts. American journal of physiology. Heart and circulatory physiology 299, H1687-1692.

Clark, N.M., Lynch, J.P., 3rd, 2011. Influenza: epidemiology, clinical features, therapy, and prevention. Seminars in respiratory and critical care medicine 32, 373-392.

Colman, P.M., Lawrence, M.C., 2003. The structural biology of type I viral membrane fusion. Nat Rev Mol Cell Biol 4, 309-319.

Cross, K.J., Langley, W.A., Russell, R.J., Skehel, J.J., Steinhauer, D.A., 2009. Composition and functions of the influenza fusion peptide. Protein and peptide letters 16, 766-778.

Das, K., Aramini, J.M., Ma, L.C., Krug, R.M., Arnold, E., 2010. Structures of influenza A proteins and insights into antiviral drug targets. Nat Struct Mol Biol 17, 530-538.

de Graaf, M., Fouchier, R.A., 2014. Role of receptor binding specificity in influenza A virus transmission and pathogenesis. The EMBO journal 33, 823-841.

Dittmann, M., Hoffmann, H.H., Scull, M.A., Gilmore, R.H., Bell, K.L., Ciancanelli, M., Wilson, S.J., Crotta, S., Yu, Y., Flatley, B., Xiao, J.W., Casanova, J.L., Wack, A., Bieniasz, P.D., Rice, C.M., 2015. A serpin shapes the extracellular environment to prevent influenza A virus maturation. Cell 160, 631-643.

Duan, S., Govorkova, E.A., Bahl, J., Zaraket, H., Baranovich, T., Seiler, P., Prevost, K., Webster, R.G., Webby, R.J., 2014. Epistatic interactions between neuraminidase mutations facilitated the emergence of the oseltamivir-resistant $\mathrm{H} 1 \mathrm{~N} 1$ influenza viruses. Nature communications 5, 5029.

El Zowalaty, M.E., Bustin, S.A., Husseiny, M.I., Ashour, H.M., 2013. Avian influenza: virology, diagnosis and surveillance. Future microbiology 8, 1209-1227.

Esumi, M., Ishibashi, M., Yamaguchi, H., Nakajima, S., Tai, Y., Kikuta, S., Sugitani, M., Takayama, T., Tahara, M., Takeda, M., Wakita, T., 2015. Transmembrane serine protease TMPRSS2 activates hepatitis C virus infection. Hepatology 61, 437-446.

Fleming, D.M., 2001. Managing influenza: amantadine, rimantadine and beyond. International journal of clinical practice 55, 189-195.

Galao, R.P., Le Tortorec, A., Pickering, S., Kueck, T., Neil, S.J., 2012. Innate sensing of HIV-1 assembly by Tetherin induces NFkappaB-dependent proinflammatory responses. Cell host \& microbe 12 , 633-644.

Garten, W., Braden, C., Arendt, A., Peitsch, C., Baron, J., Lu, Y., Pawletko, K., Hardes, K., Steinmetzer, T., Bottcher-Friebertshauser, E., 2015. Influenza virus activating host proteases: Identification, localization and inhibitors as potential therapeutics. European journal of cell biology 94, 375-383.

Garten, W., Klenk, H.D., 1983. Characterization of the carboxypeptidase involved in the proteolytic cleavage of the influenza haemagglutinin. The Journal of general virology $64(\mathrm{Pt}$ 10), 2127-2137. 
Gasi Tandefelt, D., Boormans, J., Hermans, K., Trapman, J., 2014. ETS fusion genes in prostate cancer. Endocrine-related cancer 21, R143-152.

Gierer, S., Bertram, S., Kaup, F., Wrensch, F., Heurich, A., Krämer-Kühl, A., Welsch, K., Winkler, M., Meyer, B., Drosten, C., Dittmer, U., von Hahn, T., Simmons, G., Hofmann, H., Pöhlmann, S., 2013. The spike protein of the emerging betacoronavirus EMC uses a novel coronavirus receptor for entry, can be activated by TMPRSS2, and is targeted by neutralizing antibodies. J Virol 87, 5502-5511.

Goto, H., Kawaoka, Y., 1998. A novel mechanism for the acquisition of virulence by a human influenza A virus. Proc Natl Acad Sci U S A 95, 10224-10228.

Gotoh, B., Ogasawara, T., Toyoda, T., Inocencio, N.M., Hamaguchi, M., Nagai, Y., 1990. An endoprotease homologous to the blood clotting factor $\mathrm{X}$ as a determinant of viral tropism in chick embryo. The EMBO journal 9, 4189-4195.

Govaert, T.M., Dinant, G.J., Aretz, K., Knottnerus, J.A., 1998. The predictive value of influenza symptomatology in elderly people. Family practice 15, 16-22.

Gu, R.X., Liu, L.A., Wei, D.Q., 2013. Structural and energetic analysis of drug inhibition of the influenza A M2 proton channel. Trends in pharmacological sciences 34, 571-580.

Guipponi, M., Antonarakis, S.E., Scott, H.S., 2008a. TMPRSS3, a type II transmembrane serine protease mutated in non-syndromic autosomal recessive deafness. Front Biosci 13, 1557-1567.

Guipponi, M., Toh, M.Y., Tan, J., Park, D., Hanson, K., Ballana, E., Kwong, D., Cannon, P.Z., Wu, Q., Gout, A., Delorenzi, M., Speed, T.P., Smith, R.J., Dahl, H.H., Petersen, M., Teasdale, R.D., Estivill, X., Park, W.J., Scott, H.S., 2008b. An integrated genetic and functional analysis of the role of type II transmembrane serine proteases (TMPRSSs) in hearing loss. Human mutation 29, 130-141.

Guipponi, M., Vuagniaux, G., Wattenhofer, M., Shibuya, K., Vazquez, M., Dougherty, L., Scamuffa, N., Guida, E., Okui, M., Rossier, C., Hancock, M., Buchet, K., Reymond, A., Hummler, E., Marzella, P.L., Kudoh, J., Shimizu, N., Scott, H.S., Antonarakis, S.E., Rossier, B.C., 2002. The transmembrane serine protease (TMPRSS3) mutated in deafness DFNB8/10 activates the epithelial sodium channel (ENaC) in vitro. Hum Mol Genet 11, 2829-2836.

Hamilton, B.S., Chung, C., Cyphers, S.Y., Rinaldi, V.D., Marcano, V.C., Whittaker, G.R., 2014. Inhibition of influenza virus infection and hemagglutinin cleavage by the protease inhibitor HAI-2. Biochemical and biophysical research communications 450, 1070-1075.

Hamilton, B.S., Gludish, D.W., Whittaker, G.R., 2012a. Cleavage activation of the humanadapted influenza virus subtypes by matriptase reveals both subtype and strain specificities. J Virol 86, 10579-10586.

Hamilton, B.S., Whittaker, G.R., Daniel, S., 2012b. Influenza virus-mediated membrane fusion: determinants of hemagglutinin fusogenic activity and experimental approaches for assessing virus fusion. Viruses 4, 1144-1168.

Hammonds, J., Wang, J.J., Yi, H., Spearman, P., 2010. Immunoelectron microscopic evidence for Tetherin/BST2 as the physical bridge between HIV-1 virions and the plasma membrane. PLoS Pathog 6, e1000749.

Harper, S.A., Fukuda, K., Uyeki, T.M., Cox, N.J., Bridges, C.B., Centers for Disease, C., Prevention Advisory Committee on Immunization, P., 2004. Prevention and control of influenza: recommendations of the Advisory Committee on Immunization Practices (ACIP). 
MMWR. Recommendations and reports : Morbidity and mortality weekly report. Recommendations and reports / Centers for Disease Control 53, 1-40.

Harrison, S.C., 2015. Viral membrane fusion. Virology 479-480, 498-507.

Hatesuer, B., Bertram, S., Mehnert, N., Bahgat, M.M., Nelson, P.S., Pöhlman, S., Schughart, K., 2013. Tmprss2 is essential for influenza H1N1 virus pathogenesis in mice. PLoS Pathog 9, e1003774.

Herfst, S., Imai, M., Kawaoka, Y., Fouchier, R.A., 2014. Avian influenza virus transmission to mammals. Current topics in microbiology and immunology 385, 137-155.

Herrler, G., Klenk, H.D., 1991. Structure and function of the HEF glycoprotein of influenza $\mathrm{C}$ virus. Advances in virus research 40, 213-234.

Hobson, J.P., Netzel-Arnett, S., Szabo, R., Rehault, S.M., Church, F.C., Strickland, D.K., Lawrence, D.A., Antalis, T.M., Bugge, T.H., 2004. Mouse DESC1 is located within a cluster of seven DESC1-like genes and encodes a type II transmembrane serine protease that forms serpin inhibitory complexes. J Biol Chem 279, 46981-46994.

Horimoto, T., Kawaoka, Y., 2005. Influenza: lessons from past pandemics, warnings from current incidents. Nat Rev Microbiol 3, 591-600.

Hossain, D., Bostwick, D.G., 2013. Significance of the TMPRSS2:ERG gene fusion in prostate cancer. BJU international 111, 834-835.

Huang, Y., Li, X., Zhang, H., Chen, B., Jiang, Y., Yang, L., Zhu, W., Hu, S., Zhou, S., Tang, Y., Xiang, X., Li, F., Li, W., Gao, L., 2015. Human infection with an avian influenza A (H9N2) virus in the middle region of China. Journal of medical virology 87, 1641-1648.

Igarashi, M., Ito, K., Yoshida, R., Tomabechi, D., Kida, H., Takada, A., 2010. Predicting the antigenic structure of the pandemic (H1N1) 2009 influenza virus hemagglutinin. PLoS One 5, e8553.

Jacquinet, E., Rao, N.V., Rao, G.V., Zhengming, W., Albertine, K.H., Hoidal, J.R., 2001. Cloning and characterization of the cDNA and gene for human epitheliasin. Eur J Biochem 268, 2687-2699.

Jin, H., Leser, G.P., Lamb, R.A., 1994. The influenza virus hemagglutinin cytoplasmic tail is not essential for virus assembly or infectivity. The EMBO journal 13, 5504-5515.

Jin, X., Hirosaki, T., Lin, C.Y., Dickson, R.B., Higashi, S., Kitamura, H., Miyazaki, K., 2005. Production of soluble matriptase by human cancer cell lines and cell surface activation of its zymogen by trypsin. Journal of cellular biochemistry 95, 632-647.

Kam, Y.W., Okumura, Y., Kido, H., Ng, L.F., Bruzzone, R., Altmeyer, R., 2009. Cleavage of the SARS coronavirus spike glycoprotein by airway proteases enhances virus entry into human bronchial epithelial cells in vitro. PLoS One 4, e7870.

Kawase, M., Shirato, K., van der Hoek, L., Taguchi, F., Matsuyama, S., 2012. Simultaneous treatment of human bronchial epithelial cells with serine and cysteine protease inhibitors prevents severe acute respiratory syndrome coronavirus entry. J Virol 86, 6537-6545.

Khandaker, G., Dierig, A., Rashid, H., King, C., Heron, L., Booy, R., 2011. Systematic review of clinical and epidemiological features of the pandemic influenza A (H1N1) 2009. Influenza and other respiratory viruses 5, 148-156.

Kidd, M., 2014. Influenza viruses: update on epidemiology, clinical features, treatment and vaccination. Current opinion in pulmonary medicine 20, 242-246. 
Kido, H., Okumura, Y., 2008. Msp1/Tmprss13. Front Biosci 13, 754-758.

Kido, H., Okumura, Y., Takahashi, E., Pan, H.Y., Wang, S., Yao, D., Yao, M., Chida, J., Yano, M., 2012. Role of host cellular proteases in the pathogenesis of influenza and influenza-induced multiple organ failure. Biochimica et biophysica acta 1824, 186-194.

Kido, H., Yokogoshi, Y., Sakai, K., Tashiro, M., Kishino, Y., Fukutomi, A., Katunuma, N., 1992. Isolation and characterization of a novel trypsin-like protease found in rat bronchiolar epithelial Clara cells. A possible activator of the viral fusion glycoprotein. J Biol Chem 267, 13573-13579.

Kielian, M., 2014. Mechanisms of Virus Membrane Fusion Proteins. Annual Review of Virology 1, 171-189.

Kim, T.S., Heinlein, C., Hackman, R.C., Nelson, P.S., 2006. Phenotypic analysis of mice lacking the Tmprss2-encoded protease. Mol Cell Biol 26, 965-975.

Kiso, M., Shinya, K., Shimojima, M., Takano, R., Takahashi, K., Katsura, H., Kakugawa, S., Le, M.T., Yamashita, M., Furuta, Y., Ozawa, M., Kawaoka, Y., 2010. Characterization of oseltamivir-resistant 2009 H1N1 pandemic influenza A viruses. PLoS Pathog 6, e1001079.

Knappe, S., Wu, F., Madlansacay, M.R., Wu, Q., 2004. Identification of domain structures in the propeptide of corin essential for the processing of proatrial natriuretic peptide. $\mathrm{J}$ Biol Chem 279, 34464-34471.

Krol, E., Rychlowska, M., Szewczyk, B., 2014. Antivirals--current trends in fighting influenza. Acta biochimica Polonica 61, 495-504.

Kuiken, T., 2013. Is low pathogenic avian influenza virus virulent for wild waterbirds? Proceedings. Biological sciences / The Royal Society 280, 20130990.

Kumlin, U., Olofsson, S., Dimock, K., Arnberg, N., 2008. Sialic acid tissue distribution and influenza virus tropism. Influenza and other respiratory viruses 2, 147-154.

Lang, J.C., Schuller, D.E., 2001. Differential expression of a novel serine protease homologue in squamous cell carcinoma of the head and neck. British journal of cancer 84, 237-243.

Lazarowitz, S.G., Compans, R.W., Choppin, P.W., 1973a. Proteolytic cleavage of the hemagglutinin polypeptide of influenza virus. Function of the uncleaved polypeptide HA. Virology 52, 199-212.

Lazarowitz, S.G., Goldberg, A.R., Choppin, P.W., 1973b. Proteolytic cleavage by plasmin of the HA polypeptide of influenza virus: host cell activation of serum plasminogen. Virology $56,172-180$.

Leyva-Grado, V.H., Hai, R., Fernandes, F., Belicha-Villanueva, A., Carter, C., Yondola, M.A., 2014. Modulation of an ectodomain motif in the influenza A virus neuraminidase alters tetherin sensitivity and results in virus attenuation in vivo. Journal of molecular biology 426, 1308-1321.

List, K., Szabo, R., Molinolo, A., Nielsen, B.S., Bugge, T.H., 2006. Delineation of matriptase protein expression by enzymatic gene trapping suggests diverging roles in barrier function, hair formation, and squamous cell carcinogenesis. The American journal of pathology 168, 1513-1525.

Lucas, J.M., True, L., Hawley, S., Matsumura, M., Morrissey, C., Vessella, R., Nelson, P.S., 2008. The androgen-regulated type II serine protease TMPRSS2 is differentially expressed and mislocalized in prostate adenocarcinoma. J Pathol 215, 118-125. 
Luo, M., 2012. Influenza virus entry. Advances in experimental medicine and biology 726, 201-221.

Madsen, D.H., Szabo, R., Molinolo, A.A., Bugge, T.H., 2014. TMPRSS13 deficiency impairs stratum corneum formation and epidermal barrier acquisition. Biochem J 461, 487-495.

Mair, C.M., Ludwig, K., Herrmann, A., Sieben, C., 2014. Receptor binding and pH stability how influenza A virus hemagglutinin affects host-specific virus infection. Biochimica et biophysica acta 1838, 1153-1168.

Mangeat, B., Cavagliotti, L., Lehmann, M., Gers-Huber, G., Kaur, I., Thomas, Y., Kaiser, L., Piguet, V., 2012. Influenza virus partially counteracts restriction imposed by tetherin/BST-2. J Biol Chem 287, 22015-22029.

Matrosovich, M., Stech, J., Klenk, H.D., 2009. Influenza receptors, polymerase and host range. Revue scientifique et technique 28, 203-217.

Matrosovich, M.N., Gambaryan, A.S., Tuzikov, A.B., Byramova, N.E., Mochalova, L.V., Golbraikh, A.A., Shenderovich, M.D., Finne, J., Bovin, N.V., 1993. Probing of the receptorbinding sites of the $\mathrm{H} 1$ and $\mathrm{H} 3$ influenza $\mathrm{A}$ and influenza B virus hemagglutinins by synthetic and natural sialosides. Virology 196, 111-121.

Matsuyama, S., Nagata, N., Shirato, K., Kawase, M., Takeda, M., Taguchi, F., 2010. Efficient activation of the severe acute respiratory syndrome coronavirus spike protein by the transmembrane protease TMPRSS2. J Virol 84, 12658-12664.

Medina, R.A., García-Sastre, A., 2011. Influenza A viruses: new research developments. Nat Rev Microbiol 9, 590-603.

Molinari, N.A., Ortega-Sanchez, I.R., Messonnier, M.L., Thompson, W.W., Wortley, P.M., Weintraub, E., Bridges, C.B., 2007. The annual impact of seasonal influenza in the US: measuring disease burden and costs. Vaccine 25, 5086-5096.

Murakami, M., Towatari, T., Ohuchi, M., Shiota, M., Akao, M., Okumura, Y., Parry, M.A., Kido, H., 2001. Mini-plasmin found in the epithelial cells of bronchioles triggers infection by broad-spectrum influenza A viruses and Sendai virus. Eur J Biochem 268, 2847-2855.

Muraki, Y., Hongo, S., 2010. The molecular virology and reverse genetics of influenza C virus. Japanese journal of infectious diseases 63, 157-165.

Neil, S.J., Zang, T., Bieniasz, P.D., 2008. Tetherin inhibits retrovirus release and is antagonized by HIV-1 Vpu. Nature 451, 425-430.

Nobusawa, E., Sato, K., 2006. Comparison of the mutation rates of human influenza A and B viruses. J Virol 80, 3675-3678.

Okumura, Y., Takahashi, E., Yano, M., Ohuchi, M., Daidoji, T., Nakaya, T., Böttcher, E., Garten, W., Klenk, H.D., Kido, H., 2010. Novel type II transmembrane serine proteases, MSPL and TMPRSS13, Proteolytically activate membrane fusion activity of the hemagglutinin of highly pathogenic avian influenza viruses and induce their multicycle replication. J Virol 84, 5089-5096.

Palese, P., 2004. Influenza: old and new threats.

Paoloni-Giacobino, A., Chen, H., Peitsch, M.C., Rossier, C., Antonarakis, S.E., 1997. Cloning of the TMPRSS2 gene, which encodes a novel serine protease with transmembrane, LDLRA, and SRCR domains and maps to 21q22.3. Genomics 44, 309-320. 
Paul Glezen, W., Schmier, J.K., Kuehn, C.M., Ryan, K.J., Oxford, J., 2013. The burden of influenza B: a structured literature review. American journal of public health 103, e43-51.

Potter, C.W., 2001. A history of influenza. Journal of applied microbiology 91, 572-579.

Punpanich, W., Chotpitayasunondh, T., 2012. A review on the clinical spectrum and natural history of human influenza. International journal of infectious diseases : IJID : official publication of the International Society for Infectious Diseases 16, e714-723.

Puzelli, S., Rossini, G., Facchini, M., Vaccari, G., Di Trani, L., Di Martino, A., Gaibani, P., Vocale, C., Cattoli, G., Bennett, M., McCauley, J.W., Rezza, G., Moro, M.L., Rangoni, R., Finarelli, A.C., Landini, M.P., Castrucci, M.R., Donatelli, I., Influenza Task, F., 2014. Human infection with highly pathogenic A(H7N7) avian influenza virus, Italy, 2013. Emerging infectious diseases 20, 1745-1749.

Qi, X., Jiang, J., Zhu, M., Wu, Q., 2011. Human corin isoforms with different cytoplasmic tails that alter cell surface targeting. J Biol Chem 286, 20963-20969.

Rossman, J.S., Lamb, R.A., 2011. Influenza virus assembly and budding. Virology 411, 229236.

Sakai, K., Ami, Y., Tahara, M., Kubota, T., Anraku, M., Abe, M., Nakajima, N., Sekizuka, T., Shirato, K., Suzaki, Y., Ainai, A., Nakatsu, Y., Kanou, K., Nakamura, K., Suzuki, T., Komase, K., Nobusawa, E., Maenaka, K., Kuroda, M., Hasegawa, H., Kawaoka, Y., Tashiro, M., Takeda, M., 2014. The Host Protease TMPRSS2 Plays a Major Role in In Vivo Replication of Emerging H7N9 and Seasonal Influenza Viruses. J Virol 88, 5608-5616.

Sakai, K., Sekizuka, T., Ami, Y., Nakajima, N., Kitazawa, M., Sato, Y., Nakajima, K., Anraku, M., Kubota, T., Komase, K., Takehara, K., Hasegawa, H., Odagiri, T., Tashiro, M., Kuroda, M., Takeda, M., 2015. A mutant H3N2 influenza virus uses an alternative activation mechanism in TMPRSS2 knockout mice by loss of an oligosaccharide in the hemagglutinin stalk region. J Virol 89, 5154-5158.

Saladino, R., Barontini, M., Crucianelli, M., Nencioni, L., Sgarbanti, R., Palamara, A.T., 2010. Current advances in anti-influenza therapy. Curr Med Chem 17, 2101-2140.

Sales, K.U., Hobson, J.P., Wagenaar-Miller, R., Szabo, R., Rasmussen, A.L., Bey, A., Shah, M.F., Molinolo, A.A., Bugge, T.H., 2011. Expression and genetic loss of function analysis of the HAT/DESC cluster proteases TMPRSS11A and HAT. PLoS One 6, e23261.

Sawoo, O., Dublineau, A., Batejat, C., Zhou, P., Manuguerra, J.C., Leclercq, I., 2014. Cleavage of hemagglutinin-bearing lentiviral pseudotypes and their use in the study of influenza virus persistence. PLoS One 9, e106192.

Schrauwen, E.J., de Graaf, M., Herfst, S., Rimmelzwaan, G.F., Osterhaus, A.D., Fouchier, R.A., 2014. Determinants of virulence of influenza A virus. Eur J Clin Microbiol Infect Dis 33, 479-490.

Schultz-Cherry, S., Olsen, C.W., Easterday, B.C., 2013. History of Swine influenza. Current topics in microbiology and immunology 370, 21-28.

Sedghizadeh, P.P., Mallery, S.R., Thompson, S.J., Kresty, L., Beck, F.M., Parkinson, E.K., Biancamano, J., Lang, J.C., 2006. Expression of the serine protease DESC1 correlates directly with normal keratinocyte differentiation and inversely with head and neck squamous cell carcinoma progression. Head \& neck 28, 432-440.

Shi, J., Xie, J., He, Z., Hu, Y., He, Y., Huang, Q., Leng, B., He, W., Sheng, Y., Li, F., Song, Y., Bai, C., Gu, Y., Jie, Z., 2013. A detailed epidemiological and clinical description of 6 
human cases of avian-origin influenza A (H7N9) virus infection in Shanghai. PLoS One 8, e77651.

Shirato, K., Kawase, M., Matsuyama, S., 2013. Middle East respiratory syndrome coronavirus infection mediated by the transmembrane serine protease TMPRSS2. J Virol 87, 12552-12561.

Shirato, K., Matsuyama, S., Ujike, M., Taguchi, F., 2011. Role of proteases in the release of porcine epidemic diarrhea virus from infected cells. J Virol 85, 7872-7880.

Shirogane, Y., Takeda, M., Iwasaki, M., Ishiguro, N., Takeuchi, H., Nakatsu, Y., Tahara, M., Kikuta, H., Yanagi, Y., 2008. Efficient multiplication of human metapneumovirus in Vero cells expressing the transmembrane serine protease TMPRSS2. J Virol 82, 8942-8946.

Shope, R.E., 1936. Immunization Experiments with Swine Influenza Virus. The Journal of experimental medicine 64, 47-61.

Shtyrya, Y.A., Mochalova, L.V., Bovin, N.V., 2009. Influenza virus neuraminidase: structure and function. Acta Naturae 1, 26-32.

Shulla, A., Heald-Sargent, T., Subramanya, G., Zhao, J., Perlman, S., Gallagher, T., 2011. A transmembrane serine protease is linked to the severe acute respiratory syndrome coronavirus receptor and activates virus entry. J Virol 85, 873-882.

Simmons, G., Zmora, P., Gierer, S., Heurich, A., Pohlmann, S., 2013. Proteolytic activation of the SARS-coronavirus spike protein: cutting enzymes at the cutting edge of antiviral research. Antiviral Res 100, 605-614.

Smith, W., Andrewes, C.H., Laidlaw, P.P., 1933. A VIRUS OBTAINED FROM INFLUENZA PATIENTS. The Lancet 222, 66-68.

Soema, P.C., Kompier, R., Amorij, J.P., Kersten, G.F., 2015. Current and next generation influenza vaccines: Formulation and production strategies. European journal of pharmaceutics and biopharmaceutics : official journal of Arbeitsgemeinschaft fur Pharmazeutische Verfahrenstechnik e.V 94, 251-263.

Sonnberg, S., Webby, R.J., Webster, R.G., 2013. Natural history of highly pathogenic avian influenza H5N1. Virus research 178, 63-77.

Spackman, E., 2014. A brief introduction to avian influenza virus. Methods in molecular biology 1161, 61-68.

Sriwilaijaroen, N., Suzuki, Y., 2012. Molecular basis of the structure and function of H1 hemagglutinin of influenza virus. Proceedings of the Japan Academy. Series B, Physical and biological sciences 88, 226-249.

Stieneke-Grober, A., Vey, M., Angliker, H., Shaw, E., Thomas, G., Roberts, C., Klenk, H.D., Garten, W., 1992. Influenza virus hemagglutinin with multibasic cleavage site is activated by furin, a subtilisin-like endoprotease. The EMBO journal 11, 2407-2414.

Sun, X., Jayaraman, A., Maniprasad, P., Raman, R., Houser, K.V., Pappas, C., Zeng, H., Sasisekharan, R., Katz, J.M., Tumpey, T.M., 2013. N-linked glycosylation of the hemagglutinin protein influences virulence and antigenicity of the 1918 pandemic and seasonal H1N1 influenza A viruses. J Virol 87, 8756-8766.

Sun, X., Whittaker, G.R., 2013. Entry of influenza virus. Landes Biosciences and Springer Science + Business Media. 
Suzuki, Y., Ito, T., Suzuki, T., Holland, R.E., Chambers, T.M., Kiso, M., Ishida, H., Kawaoka, Y., 2000. Sialic acid species as a determinant of the host range of influenza A viruses. J Virol 74, 11825-11831.

Szabo, R., Bugge, T.H., 2011. Membrane-anchored serine proteases in vertebrate cell and developmental biology. Annual review of cell and developmental biology 27, 213-235.

Szabo, R., Netzel-Arnett, S., Hobson, J.P., Antalis, T.M., Bugge, T.H., 2005. Matriptase-3 is a novel phylogenetically preserved membrane-anchored serine protease with broad serpin reactivity. Biochem J 390, 231-242.

Tarnow, C., Engels, G., Arendt, A., Schwalm, F., Sediri, H., Preuss, A., Nelson, P.S., Garten, W., Klenk, H.D., Gabriel, G., Bottcher-Friebertshauser, E., 2014. TMPRSS2 is a host factor that is essential for pneumotropism and pathogenicity of H7N9 influenza A virus in mice. $J$ Virol 88, 4744-4751.

Thorlund, K., Awad, T., Boivin, G., Thabane, L., 2011. Systematic review of influenza resistance to the neuraminidase inhibitors. BMC infectious diseases 11, 134.

Tong, S., Li, Y., Rivailler, P., Conrardy, C., Castillo, D.A., Chen, L.M., Recuenco, S., Ellison, J.A., Davis, C.T., York, I.A., Turmelle, A.S., Moran, D., Rogers, S., Shi, M., Tao, Y., Weil, M.R., Tang, K., Rowe, L.A., Sammons, S., Xu, X., Frace, M., Lindblade, K.A., Cox, N.J., Anderson, L.J., Rupprecht, C.E., Donis, R.O., 2012. A distinct lineage of influenza A virus from bats. Proc Natl Acad Sci U S A 109, 4269-4274.

Tong, S., Zhu, X., Li, Y., Shi, M., Zhang, J., Bourgeois, M., Yang, H., Chen, X., Recuenco, S., Gomez, J., Chen, L.M., Johnson, A., Tao, Y., Dreyfus, C., Yu, W., McBride, R., Carney, P.J., Gilbert, A.T., Chang, J., Guo, Z., Davis, C.T., Paulson, J.C., Stevens, J., Rupprecht, C.E., Holmes, E.C., Wilson, I.A., Donis, R.O., 2013. New world bats harbor diverse influenza A viruses. PLoS Pathog 9, e1003657.

Trebbien, R., Larsen, L.E., Viuff, B.M., 2011. Distribution of sialic acid receptors and influenza A virus of avian and swine origin in experimentally infected pigs. Virology journal 8,434 .

Tregoning, J.S., Schwarze, J., 2010. Respiratory viral infections in infants: causes, clinical symptoms, virology, and immunology. Clinical microbiology reviews 23, 74-98.

Tsubamoto, Y., Yamada, N., Watanabe, Y., Inaba, T., Shiomi, M., Shimano, H., Gotoda, T., Harada, K., Shimada, M., Ohsuga, J., et al., 1994. Dextran sulfate, a competitive inhibitor for scavenger receptor, prevents the progression of atherosclerosis in Watanabe heritable hyperlipidemic rabbits. Atherosclerosis 106, 43-50.

Tumpey, T.M., Basler, C.F., Aguilar, P.V., Zeng, H., Solorzano, A., Swayne, D.E., Cox, N.J., Katz, J.M., Taubenberger, J.K., Palese, P., Garcia-Sastre, A., 2005. Characterization of the reconstructed 1918 Spanish influenza pandemic virus. Science 310, 77-80.

Uhlen, M., Fagerberg, L., Hallstrom, B.M., Lindskog, C., Oksvold, P., Mardinoglu, A., Sivertsson, A., Kampf, C., Sjostedt, E., Asplund, A., Olsson, I., Edlund, K., Lundberg, E., Navani, S., Szigyarto, C.A., Odeberg, J., Djureinovic, D., Takanen, J.O., Hober, S., Alm, T., Edqvist, P.H., Berling, H., Tegel, H., Mulder, J., Rockberg, J., Nilsson, P., Schwenk, J.M., Hamsten, M., von Feilitzen, K., Forsberg, M., Persson, L., Johansson, F., Zwahlen, M., von Heijne, G., Nielsen, J., Ponten, F., 2015. Proteomics. Tissue-based map of the human proteome. Science 347, 1260419.

van Albada, S.B., ten Wolde, P.R., 2007. Enzyme localization can drastically affect signal amplification in signal transduction pathways. PLoS computational biology 3, 1925-1934. 
Van Damme, N., Goff, D., Katsura, C., Jorgenson, R.L., Mitchell, R., Johnson, M.C., Stephens, E.B., Guatelli, J., 2008. The interferon-induced protein BST-2 restricts HIV-1 release and is downregulated from the cell surface by the viral Vpu protein. Cell host \& microbe $3,245-252$.

Wang, C.Y., Meynard, D., Lin, H.Y., 2014. The role of TMPRSS6/matriptase-2 in iron regulation and anemia. Frontiers in pharmacology 5, 114.

Watanabe, R., Leser, G.P., Lamb, R.A., 2011. Influenza virus is not restricted by tetherin whereas influenza VLP production is restricted by tetherin. Virology 417, 50-56.

Webster, R.G., Sharp, G.B., Claas, E.C., 1995. Interspecies transmission of influenza viruses. American journal of respiratory and critical care medicine 152, S25-30.

White, J.M., Delos, S.E., Brecher, M., Schornberg, K., 2008. Structures and mechanisms of viral membrane fusion proteins: multiple variations on a common theme. Critical reviews in biochemistry and molecular biology 43, 189-219.

Wilks, S., de Graaf, M., Smith, D.J., Burke, D.F., 2012. A review of influenza haemagglutinin receptor binding as it relates to pandemic properties. Vaccine 30, 4369-4376.

Winkler, M., Bertram, S., Gnirß, K., Nehlmeier, I., Gawanbacht, A., Kirchhoff, F., Ehrhardt, C., Ludwig, S., Kiene, M., Moldenhauer, A.S., Goedecke, U., Karsten, C.B., Kühl, A., Pöhlmann, S., 2012. Influenza A virus does not encode a tetherin antagonist with Vpu-like activity and induces IFN-dependent tetherin expression in infected cells. PLoS One 7, e43337.

Worch, R., 2014. Structural biology of the influenza virus fusion peptide. Acta biochimica Polonica 61, 421-426.

Yamaguchi, M., Danev, R., Nishiyama, K., Sugawara, K., Nagayama, K., 2008. Zernike phase contrast electron microscopy of ice-embedded influenza A virus. Journal of structural biology 162, 271-276.

York, I., Donis, R.O., 2013. The 2009 pandemic influenza virus: where did it come from, where is it now, and where is it going? Current topics in microbiology and immunology 370, 241-257.

Zhang, J., Leser, G.P., Pekosz, A., Lamb, R.A., 2000. The cytoplasmic tails of the influenza virus spike glycoproteins are required for normal genome packaging. Virology 269, 325-334.

Zhang, Y., Liu, Q., Wang, D., Chen, S., Wang, S., 2013. Simultaneous detection of oseltamivir- and amantadine-resistant influenza by oligonucleotide microarray visualization. PLoS One 8, e57154.

Zhirnov, O.P., Ikizler, M.R., Wright, P.F., 2002. Cleavage of influenza a virus hemagglutinin in human respiratory epithelium is cell associated and sensitive to exogenous antiproteases. $\mathrm{J}$ Virol 76, 8682-8689.

Zhirnov, O.P., Klenk, H.D., Wright, P.F., 2011. Aprotinin and similar protease inhibitors as drugs against influenza. Antiviral Res 92, 27-36.

Zhou, Y., Vedantham, P., Lu, K., Agudelo, J., Carrion, R., Jr., Nunneley, J.W., Barnard, D., Pohlmann, S., McKerrow, J.H., Renslo, A.R., Simmons, G., 2015. Protease inhibitors targeting coronavirus and filovirus entry. Antiviral Res 116, 76-84.

Zmora, P., Pöhlmann, S., 2015. New approaches to influenza therapy. In: Ed. Mendez-Vilas, A. The Battle Against Microbial Pathogens: Basic Science, Technological Advances and Educational Programs, accepted 


\section{List of abbreviations}

AIDS acquired immunodeficiency syndrome

BST-2 bone marrow stromal cell antigen 2

$\mathrm{CoV} \quad$ coronavirus

CUB Cls/Clr, urchin embryonic growth factor and bone morphogenetic proten-1

DESC1 differentialy expressed in squamous cell carcinoma 1

E glutamic acid

ER endoplasmatic reticulum

F phenylalanine

FLUAV influenza A virus

FLUBV influenza B virus

FLUCV influenza $\mathrm{C}$ virus

$\mathrm{Fz} \quad$ frizzled domain

G glycine

GPI glycosyl-phospatidylinositol

HA hemagglutinin

$\mathrm{HA}_{1} \quad$ hemagglutinin 1 subunit

$\mathrm{HA}_{2} \quad$ hemagglutinin 2 subunit

HAT human airway trypsin-like protease

HIV human immunodeficiency virus

HPAIV highly pathogenic avian influenza virus

K lysine

L leucine

LDLRA low-density lipoprotein receptor class A domain

LPAIV low pathogenic avian influenza virus

M1 matrix 1 protein

M2 matrix 2 protein

MAM meprin, A5 antigen, and receptor protein phosphatase $\mu$ domain

MERS Middle East respiratory syndrome

MmM Minimotif Miner

MSPL mosaic serine protease long-form

$\mathrm{N} \quad$ asparagine

NA neuraminidase

NEP nuclear export protein 
nucleoprotein

NS1

non-structural protein 1

NS2

non-structural protein 2

PA

polymerase acidic protein

PB1

polymerase basic protein 1

PB2

polymerase basic protein 2

PCC Pearson correlation coefficient

PEDV porcine epidemic diarrhea virus

PICS proteomic identification of protease cleavage sites

pro-ANP pro-atrial natriuretic peptide

$\mathrm{R}$

serine

RNA ribonucleic acid

S

spike

SARS severe acute respiratory syndrome

SEA sea urchin sperm protein/enteropeptidase/agrin

SR Group A scavenger receptor domain

SR-A scavenger receptor type $\mathrm{A}$

$\mathrm{T}$

threonine

TM transmembrane domain

TTSP type II transmembrane serine protease

USD United States dollar

vRNP viral ribonucleoprotein

WHO World Health Organization 


\section{Acknowledgements}

First of all, I would like to express my gratitude to Prof. Stefan Pöhlmann for giving me the opportunity to join his team and work on this amazing project! Many thanks for valuable comments, constructive critic, never saying 'no' and letting me to perform every experiment which was born in my head.

I would like to thank Prof. Dr. Dr. Klaus-Peter Czerny and Dr. Mikhail Matrosovich for participating in Thesis Advisory Committee and for helpful advice and interesting discussions. Additionally, I would like to thank Prof. Dr. Dr. Klaus-Peter Czerny for his willingness to serve as a Referee.

Many thanks to all members of the Infection Biology Unit, German Primate Center. I really appreciate friendly atmosphere, your kindness, all good and bad moments, all the talks about science and about everything except of science as well as every smile and laugh. Special thanks to Dr. Stephanie Bertram, who showed me fascinating world of HA proteolytic cleavage. Additionally, I would like to express my gratitude to Anna-Sophie Moldenhauer for her excellent technical help. Moreover, I would like to thank Dr. Markus Hoffmann for his

valuable comments regarding this thesis. Many, many thanks to Kathrin Habenicht for all paper work and her invaluable help at the beginning of my stay in Germany. And last, but not least, very special thanks to Adeline Heurich (for dancing Polka, for all crazy talks, for her smile and willingness to laugh), as well as Anika Lins and Constantin Brinkmann (for our talks after 3 p.m., unicorns, and fun which I had with both of you in the lab).

Further, I would like to thank Gottingen Graduate School for Neuroscience, Biophysics and Molecular Biosciences and Leibniz Graduate School for Emerging Infectious Diseases for financial support.

I would like to express special thanks to my friends. Szymon, thank you for everything and for every moment when you showed me that there is a fantastic world outside of the lab! Łukasz, thank you that I can always call you and complain, thanks for every cup of tea, and special thanks for your help with computers! Agnieszka, thank you for our talks and every email you wrote!

Specjalne podziękowania dla mojej Rodziny. Bez Was nie byłoby mnie tu, gdzie teraz jestem. Dziękuję za Wasze wsparcie, troskę i wiarę we mnie! 


\section{Curriculum vitae}

\section{PERSONAL DETAILS}

Name:

Date of birth:

Place of birth:

Nationality:

EDUCATION

2012 - present

$2005-2010$
Pawel Zmora

$4^{\text {th }}$ October 1986

Szprotawa, Poland

Polish
Georg-August-University Götingen, Göttingen, GERMANY

Leibniz Graduate School for Emerging Infectious Diseases

Goettingen Graduate School for Neurosciences, Biophysics and Molecular Biosciences

PhD, Emerging Infectious Diseases

Thesis: Activation of the influenza virus hemagglutinin by type II transmembrane serine proteases

Supervisor: $\quad$ Prof. Dr. Stefan Pöhlmann

Poznan University of Life Sciences, Poznan, POLAND

MSc Eng., Biotechnology

Thesis: Effect of the long chain fatty acids on the rumen methanogens population

Supervisor: $\quad$ Prof. Dr. Adam Cieslak

Final grade: 5.0 (very good)

\section{HONOURS AND AWARDS}

2013 - present
$2008-2010 \quad \begin{aligned} & \text { The GGNB Excellence Stipend } \\ & \text { the academic achievement }\end{aligned}$
The Scholarship of the Science Foundation Ireland (SFI)

\section{RESEARCH EXPERIENCE}

2012 - present

$2010-2012$
Infection Biology Unit, German Primate Center Leibniz Institute for Primate Research, Göttingen, GERMANY

Doctoral Candidate; Advisor: Prof. Dr. Stefan Pöhlmann

'The proteolytic activation of influenza virus'

RUMEN PULS, Department of Animal Nutrition and Feed Management, Poznan University of Life Sciences, Poznan, POLAND

Research Assistant; Advisor: Prof. Dr. Malgorzata Szumacher-Strabel

'The modulation of methane emission in the rumen environment' 
RUMEN PULS, Department of Animal Nutrition and Feed Management, Poznan University of Life Sciences, Poznan, POLAND

Graduate Student; Advisor: Prof. Dr. Adam Cieslak

'The diet supplementation with long chain fatty acids and its effect on the methanogenesis'

$2008-2008$

Department of Microbiology, National University of Ireland, Galway, IRELAND

Infectious Diseases Undergraduate Research Experience \& Knowledge Award Programme; Advisor: Aoife Boyd, PhD

'Complementation of Type Three Secretion System (TTSS) mutant strains of Vibrio parahaemolyticus"

2007 - 2008 Department of Pharmacology and Biotechnology, Research Institute on Medical Plants, Poznan, POLAND

Research Assistant; Advisor: Anna Krajewska-Patan, $\mathrm{PhD}$

'The effect of microbial secondary metabolites on the growth and metabolic activity of in vitro plant tissue culture'

$2006-2007 \quad$ Molecular Diagnostics Laboratory, Department of Biochemistry and Biotechnology, Poznan University of Life Sciences, Poznan, POLAND Intern Student; Advisor: Mariola Galbas, $\mathrm{PhD}$

'Inhibition of pathogenic bacteria by the microorganisms isolated from the silages'

\section{RELATED PROFESSIONAL EXPERIENCE}

$2013-2014$

Central European Journal of Biology

Associate Editor

\section{PROFESSIONAL ASSOCIATIONS}

$2007-2010$

$2005-2010$
Academic Society of Biotechnology Students

- 2007-2009-Chairman

Scientific Society of Biotechnology Students OPERON

- 2007 - 2009 - Chairman 


\section{PEER-REVIEWED PUBLICATIONS}

Zmora. P., Moldenhauer, A.S., Pöhlmann, S. (2015) TMPRSS2 isoforms 1 activates respiratory viruses and is expressed in viral target cells. PLOS ONE 10: e0138380

Gnirß, K.*, Zmora, P.*, Blazejewska, P., Winkler, M., Lins, A., Nehlmeier, I., Gärtner, S., Moldenhauer, A., Hofmann-Winkler, H., Wolff, T., Schindler, M., Pöhlmann, S. (2015) Tetherin sensitivity of influenza A viruses is strain specific: Role of hemagglutinin and neuraminidase. Journal of Virology 89:9178-88 *equal contribution

Zmora, P., Blazejewska, P., Moldenhauer, A.S., Welsch, K., Nehlmeier, I., Wu, Q., Schneider, H., Pöhlmann, S., Bertram, S. (2014) DESC1 and MSPL Activate Influenza A Viruses and Emerging Coronaviruses for Host Cell Entry. Journal of Virology 88:12087-97

Zmora. P., Moldenhauer, A.S., Pöhlmann, S. The stem domain of type II transmembrane serine proteases determines the activation of viral glycoproteins and is a potential target for intervention. Journal of Virology (submitted)

Krämer-Kühl, A.*, Zmora, P.*, Blazejewska, P.*, Gnirß, K.*, Nehls, J., Bertram, S., Dijkman, R., Heurich, A., Karsten, C.B., Winkler, M., Wolff, T., Thiel, V., Schindler, M., Pöhlmann, S. Influenza virus activating host cell proteases antagonize tetherin. Journal of Virology (submitted); *equal contribution

\section{REVIEW ARTICLES AND BOOK CHAPTERS}

Simmons, G., Zmora, P., Gierer, S., Heurich, A., Pöhlmann, S. (2013) Proteolytic activation of the SARS-coronavirus spike protein: cutting enzymes at the cutting edge of antiviral research. Antiviral Research 100(3):605-1

Zmora, P., Pöhlmann, S. (2015) New approaches to influenza therapy. In book: The Battle Against Microbial Pathogens: Basic Science, Technological Advances and Educational Programs, Publisher: Formatex Research Center, Editors: A. Méndez-Vilas, accepted

Zmora, P., Pöhlmann, S. (2014) Microscopy as a useful tool to study the proteolytic activation of influenza viruses. In book: Microscopy: advances in scientific research and education, Publisher: Formatex Research Center, Editors: A. Méndez-Vilas, pp.725-731

\section{CONFERENCE PRESENTATIONS}

Zmora, P., Moldenhauer, A.S., Pöhlmann, S. (2015) TMPRSS11a activates influenza viruses and emerging coronaviruses. Oral presentation delivered at the National Symposium on Zoonoses Research, Berlin, Germany

Zmora, P., Moldenhauer, A.S., Pöhlmann, S. (2015) The stem region of type II transmembrane serine proteases is a determinant of influenza virus activation. Oral presentation delivered at the Influenza 2015, Oxford, UK

Zmora, P., Moldenhauer, A.S., Pöhlmann, S. (2015) The stem region of type II transmembrane serine proteases can determine activation of influenza virus and emerging coronaviruses. Oral presentation delivered at the 25th Annual Meeting of the Society for Virology, Bochum, Germany

Zmora, P., Blazejewska, P., Moldenhauer, A.S., Nehlmeier, I., Wu, Q., Velasco, G., Bertram, S., Pöhlmann, S. (2014) Type II transmembrane serine proteases MSPL and DESC1 activate influenza viruses and emerging coronaviruses. Poster presentation delivered at the Keystone Symposia Pathogenesis of Respiratory Viruses, Keystone, USA 